\title{
EVALUATION OF ELECTROCHEMICAL AND LASER POLISHING OF SELECTIVELY LASER MELTED 316L STAINLESS STEEL
}

A Thesis

presented to

the Faculty of California Polytechnic State University,

San Luis Obispo

\author{
In Partial Fulfillment \\ of the Requirements for the Degree \\ Master of Science in Industrial Engineering
}

by

Julian Lohser

June 2018 
(C) 2018

Julian Lohser

ALL RIGHTS RESERVED 
TITLE: Evaluation of Electrochemical and Laser

Polishing of Selectively Laser Melted 316L

Stainless Steel

AUTHOR: Julian Lohser

DATE SUBMITTED: June 2018

COMMITTEE CHAIR: Xuan Wang, Ph.D.

Associate Professor of Industrial and

Manufacturing Engineering

COMMITTEE MEMBER: Dan Walsh, Ph.D.

Professor Emeritus of Materials Engineering

COMMITTEE MEMBER: Thomas Pluschkell, M.S.

Mechanical Engineer at Lawrence Livermore

National Laboratory 


\begin{abstract}
Evaluation of Electrochemical and Laser Polishing of Selectively Laser Melted 316L Stainless Steel

Julian Lohser
\end{abstract}

Selective laser melting has shown incredible growth as a metallic additive manufacturing process in recent years. While it does provide many solutions and new ways to approach challenges, it does not come without issues of its own, namely, surface roughness. In the as-printed state, the surface roughness of selectively laser melted parts is unacceptable for use in engineering applications. Additionally, selective laser melting is used to produce complex geometries with hard to reach features, preventing conventional mechanical polishing from being successful. Therefore, it is necessary to evaluate non-mechanical polishing processes as treatments for surface roughness. In this study, electrochemical and laser polishing were investigated as potential start-to-finish treatments for the surface roughness of selectively laser melted parts. Following this preliminary study, a follow-up study investigating the effect on the mechanical strength of a lattice design that electropolishing would have. Electropolishing was found to significantly reduce the surface roughness of the as-printed part, but not to a usable value. Additionally, electropolishing was found to be unacceptable for use on lattice parts. Laser polishing was found to significantly reduce the surface roughness of the part but had feature size issues preventing a perfectly smooth surface.

Keywords: selective laser melting, laser polishing, electrochemical polishing, lattice, metallurgy 


\section{ACKNOWLEDGMENTS}

First, I would like to thank Dr. Wang for allowing me to do this thesis and providing all of his knowledge and skills when relating to 3-d printing. You have provided me with more opportunities in life than I could have expected.

Secondly, I would like to thank Tommy Pluschkell and the team at Lawrence Livermore National Labs for providing the SLM 125 to make this thesis possible in the first place. I enjoyed learning about real life applications for AM processed hardware, as it made contextualizing the thesis that much easier.

Finally, special thanks to Rhys Gilmore for doing a similar thesis as me and bringing out my competitive nature, causing me to do better work than I ever would have done on my own. 


\section{TABLE OF CONTENTS}

Page

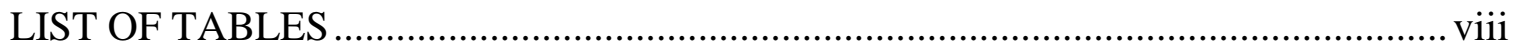

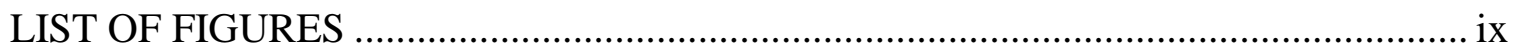

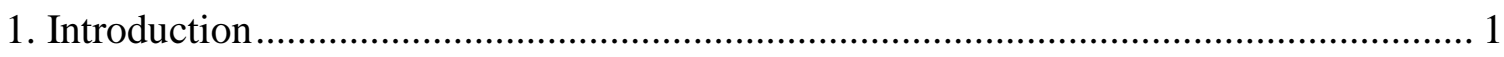

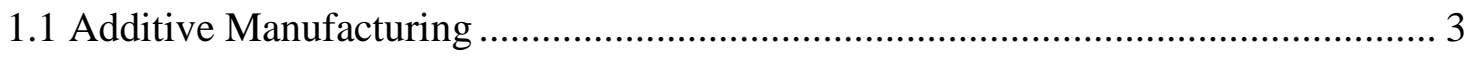

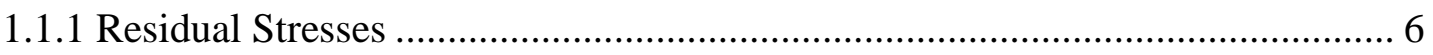

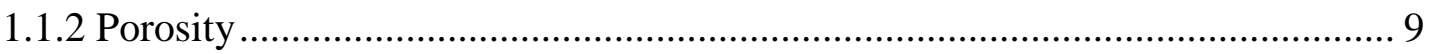

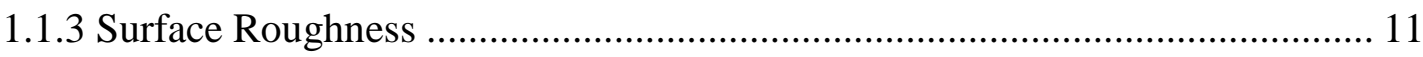

1.2 Surface Treatments..................................................................................... 16

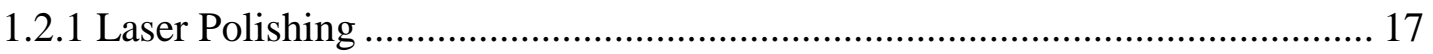

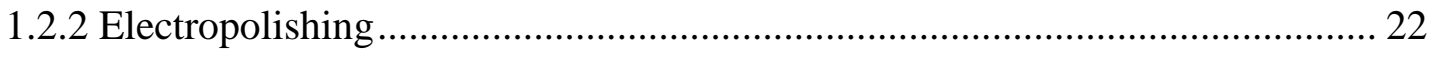

1.3 Solidification Structures and other Material Properties .................................... 26

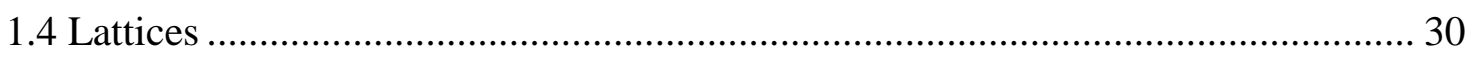

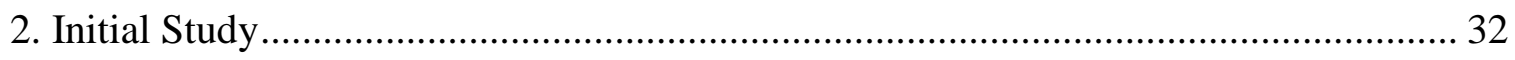

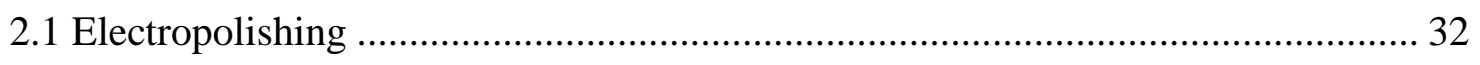

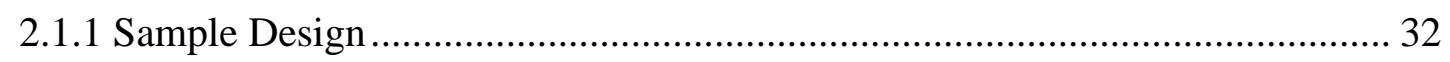

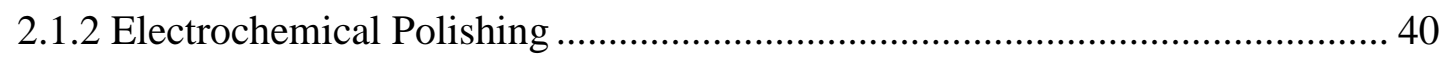

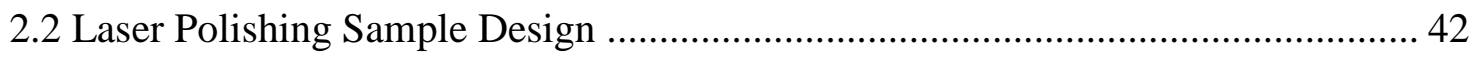

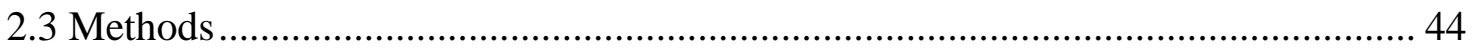

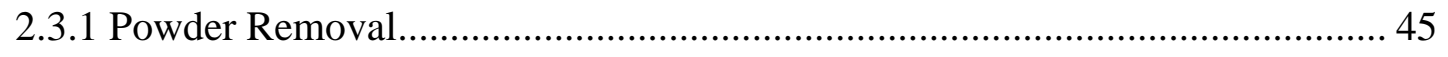

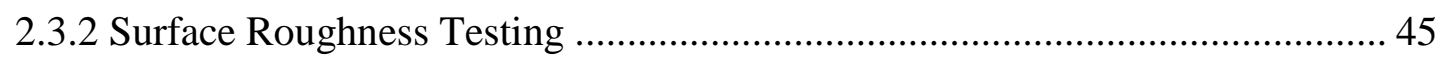

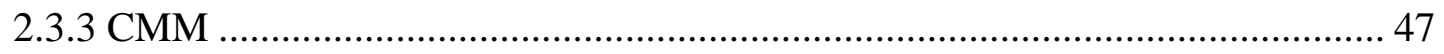

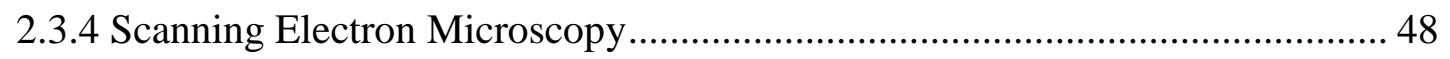

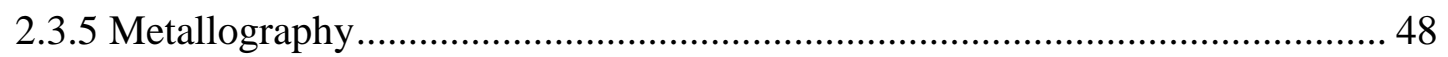




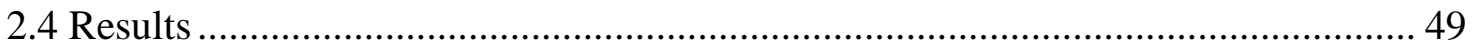

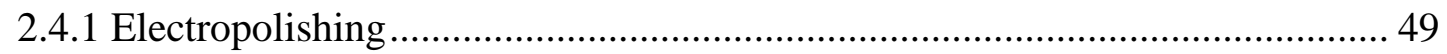

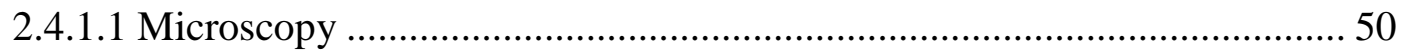

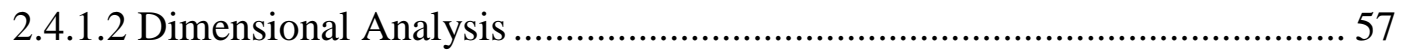

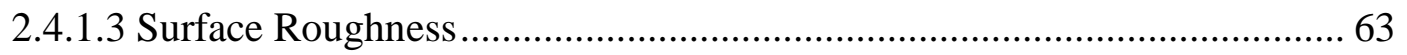

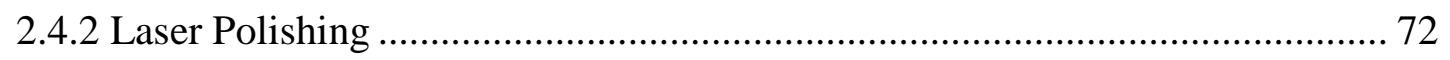

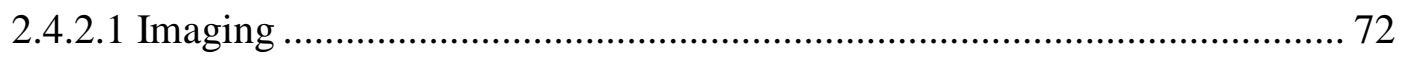

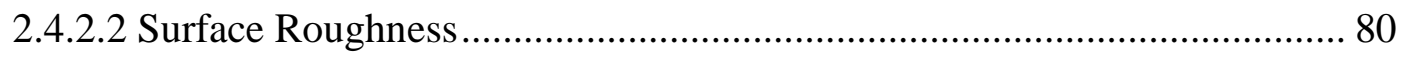

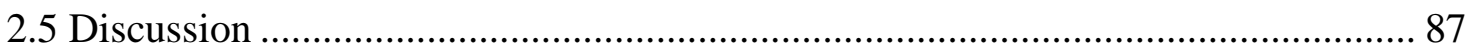

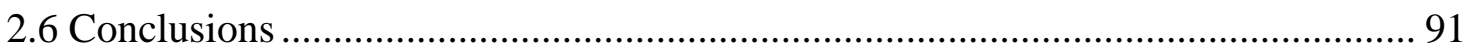

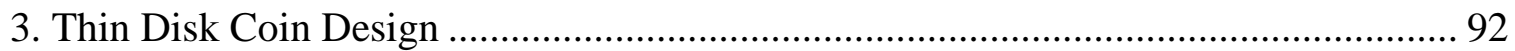

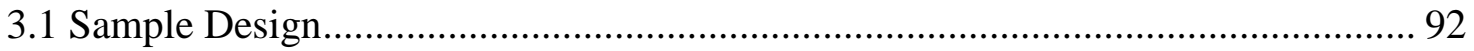

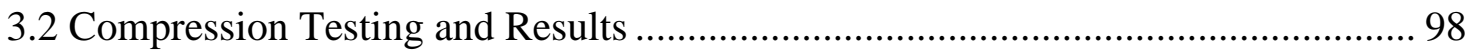

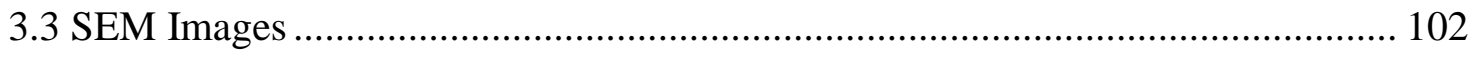

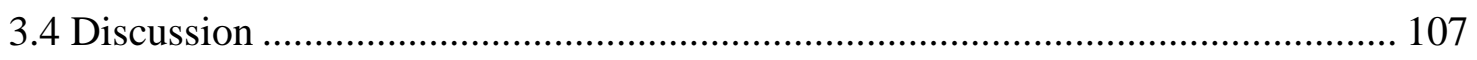

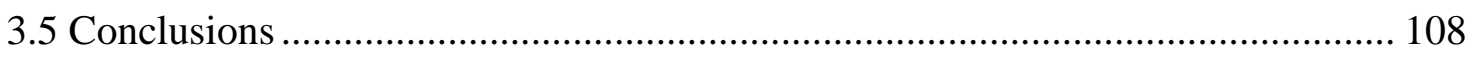

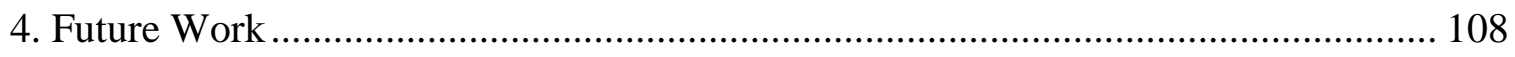

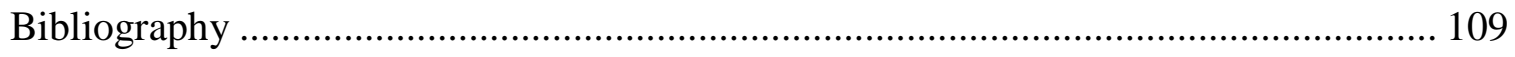

Appendices

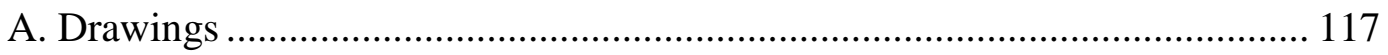

B. SLM Laser Parameters .............................................................................. 118

C. Raw Measurement Data …………………………................................ 120 


\section{LIST OF TABLES}

Table

Page

1. Common Additive Manufacturing Alloys and Applications

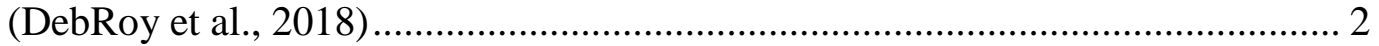

2. Laser Polishing Key Parameters (Willenborg, 2011) ....................................... 22

3. Electropolishing Process Parameters .......................................................... 41

4. Laser Polishing Experimental Parameters ......................................................... 44

5. Experimental Design for Polishing of Thin Disk Coin Designs ......................... 96

6. Hardness Measurements of Thin Disk Samples ................................................. 98

7. Summarized Compression Test Data for Thin Disk Lattice Samples ................ 101

8. Mass Loss Measurements for Electropolished Thin Disk Lattice Samples........ 102 


\section{LIST OF FIGURES}

Figure $\quad$ Page

1. Differentiation of metal based AM processes (Schmidt et al., 2017)................... 4

2. Summary of important components in the selective laser melting process

(Schmidt et al., 2017). 5

3. Residual stress along longitudinal direction at the end of deposition of (a) $2^{\text {nd }}$

(b) $4^{\text {th }}(\mathrm{c}) 6^{\text {th }}(\mathrm{d}) 8^{\text {th }}$ and (e) $10^{\text {th }}$ layer of IN 718 powder on IN 718 substrate

(Mukherjee et al., 2017) 7

4. Visualization of porosity in selectively laser melted cylinder by $\mathrm{x}$-ray

microtomography (Tammas-Williams et al., 2016).

5. Schematic of surface error caused by stair step effect on an inclined surface (Strano et al., 2013)

6. First kind of balling phenomenon caused by limited liquid formation (Gu \& Shen, 2009).

7. SEM images of $316 \mathrm{~L}$ SS balling phenomenon (a) with no sintering neck (b) with sintering neck (Gu \& Shen, 2009).

8. Depiction of change in molten pool geometry and size as laser scan speed is increased at given laser power ( $\mathrm{Gu} \&$ Shen, 2009).

9. SEM characterization of 316L SS second balling phenomenon at different scan speeds (a) shows similar formation to first balling phenomenon (b) is a higher speed (c) is a magnification of (b) (d) is an even higher scan rate (Gu \& Shen, 2009).

10. Visual Comparison of three different laser polishing processes (Willenborg, 2011).

11. The six main effects preventing surface tension from producing a perfectly smooth surface (Willenborg, 2011).

12. The boundary between as selectively laser melted and laser micro polished TC4 Ti alloy (C. P. Ma, Guan, \& Zhou, 2017).

13. Hardness measurements of laser polished $316 \mathrm{~L}$ at different energy densities and polishing environments (Bhaduri et al., 2017). 
14. Microstructure of etched $316 \mathrm{~L}$ following laser polishing at (a) $20 \mathrm{~J} / \mathrm{cm}^{2}$ in air

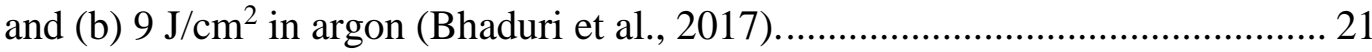

15. Different surface finishes of $316 \mathrm{SS}$ as a function of current density and overpotential (Gomez-Gallegos et al., 2016) 24

16. Schematic showing the flattening process of electrochemical processing of pure titanium (Jung et al., 2017). 25

17. Grain structure produced as a function of undercooling due to increasing scan speed (DebRoy et al., 2018). 27

18. Three layers of additively manufactured $316 \mathrm{~L}$ showing microstructural features (Herzog et al., 2016). 28

19. Thermal gradient versus cooling rate and its effect on grain morphology and size (DebRoy et al., 2018)

20. A handful of different unit cell types used for lattice structures (Merkt, Hinke, Bültmann, Brandt, \& Xie, 2015) 30

21. Characteristic stress-strain curves of (Left) bending-dominated and (Right) stretch dominated lattice structures (Recep Gümrük et al., 2013).

22. The first iteration of the double hexagon design, featuring a single, straight, hexagonal through hole.

23. (Left) Stacked hexagon with a series of bent holes. (Right) Stacked hexagon with holes that taper into a cavity.

24. Stacked hexagon design with a variety of diamond through holes and a series of decreasing diameter circular through holes. ........................................................ 34

25. Section view of internal cavities of hexagon design.......................................... 34

26. Labeling convention for the various holes for the electropolishing process evaluation coupon.

27. Magics output of three stacked hexagons and an early iteration of the laser polishing sample. 36

28. SEM image of a sample of the powder used for all of the builds. 37

29. (Left) Failed build in build chamber. (Right) Failed build after removal from build chamber showing warpage, significant burn in, and missing material. 38 
30. First successful build, still attached to build plate. Some cracking of the support structure can be seen. ................................................................... 39

31. Unfinished build still attached to build plate after removal from build chamber. 40

32. Orientation of hexagon during electropolishing. The electrolyte flowed out of the holes.

33. Front view and top view of hexagon immediately following electropolishing

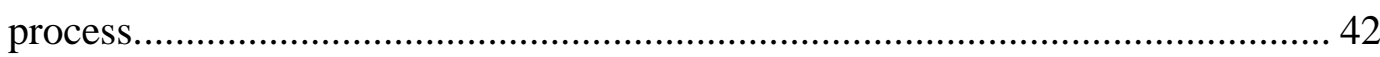

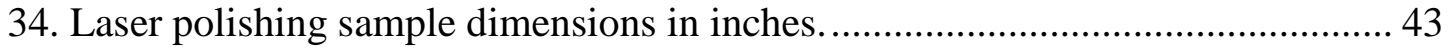

35. Laser polishing sample still attached to the build plate with a hexagon in the background.

36. Immediately following support structure removal, the sample was ultrasonicated. 45

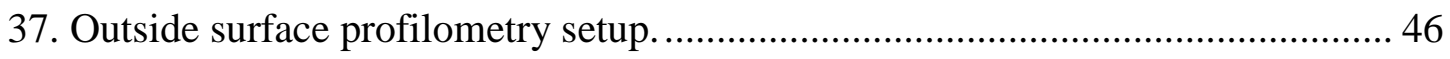

38. Profilometer setup for interior surface roughness measurements....................... 46

39. Hexagon immediately following sectioning. ................................................ 47

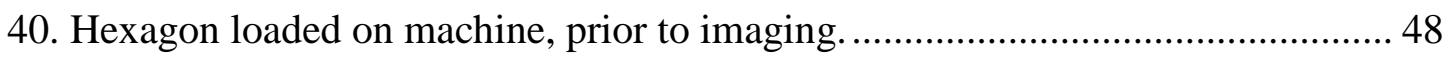

41. Multiple CMM images stitched together, depicting a single image's

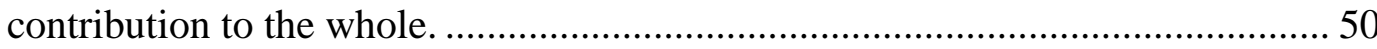

42. CMM image of a corner of electropolished hexagon showing an apparent

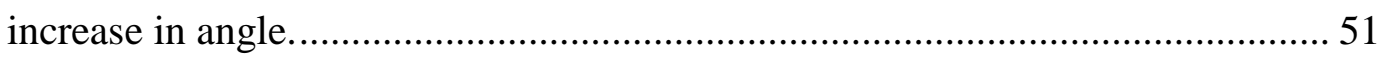

43. The difference between the two polished surfaces. ....................................... 51

44. CMM image of the top left and top right corners of the electropolished hexagon.

45. 83x magnification SEM image of an electropolished corner, showing a clean exterior surface with a powder covered interior surface

46. (Left) 100x SEM image with apparent powder particles remaining. (Right) 162x SEM image showing tear out at corner.

47. SEM image of $6 \mathrm{~mm}$ hole immediately following electropolishing; large parasitic powder deposits are clearly visible. 
48. Sectioned interior of two largest diameter through holes of electropolished hexagon, showing lack of penetration of polishing. Electrolyte entered the holes at the bottom of this picture and flowed out the top.

49. SEM images of upward facing surfaces of (Left) $5 \mathrm{~mm}$ hole at 48x and (Right) $4 \mathrm{~mm}$ hole at $53 \mathrm{x}$. 55

50. SEM images of etched microstructure, parallel to the build direction. (Left) 3122x magnification, showing both transverse and in-plane columnar growth. (Right) 3250x of an undercooling spine. 56

51. SEM images of etched microstructure, perpendicular to the build direction.

High magnification image shows sub $5 \mu \mathrm{m}$ sub-grain size. 57

52. Labeling convention for corners of the through holes. 58

53. Interval plots by hole position and hole number for the measured angle (deg) and radii $(\mathrm{mm})$ of each corner. 58

54. Boxplots of electropolished versus as-printed measured radius and corner angle.

55. ANOVA output for electropolished radius measurements, showing strong significance for the interaction between hole and corner.

56. Four-in-one plot for electropolished radius measurements. 60

57. ANOVA output for electropolished angle measurements, showing significance of hole and corner interaction. 60

58. Four-in-one plot for electropolished angle measurements. 61

59. Hypothesis and test results for two-sample t-test for the difference in means between electropolished and as-printed hole distances, showing significance..... 62

60. Descriptive statistics of as-printed and electropolishing distance measurements. Units in millimeters.

61. Labeling convention for surfaces measured by profilometer.

62. Boxplot comparing average measured surface roughness of as printed (AP) and electropolished (EP) holes, broken down by specific edge.

63. ANOVA table for average measured surface roughness of the electropolished hexagon, using hole and edge as factors. The interaction between hole and edge was found to be significant. 
64. Four-in-one residual plot for measured surface roughness of electropolished

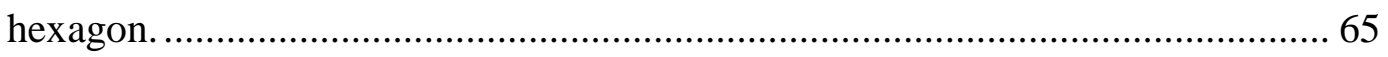

65. Interaction plot of hole and corner for the electropolished hexagon. .................... 65

66. Descriptive statistics for average surface roughness by hole for the

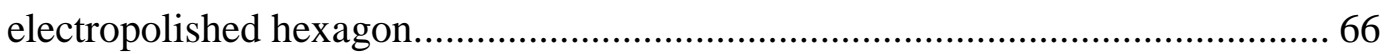

67. Multiple comparisons for the mean surface roughness by edge.......................... 67

68. ANOVA table for surface roughness measurements of as-printed interior surfaces; the interaction of hole and edge was found to be significant. ............... 67

69. Residual plots for the average surface roughness measurements of as-printed hexagon; no indication of deviation from ANOVA assumptions........................... 68

70. Interaction plot for measured surface roughness of as-printed part....................... 69

71. ANOVA results for measured surface roughness, considering surface and processing history as factors. Both factors were independently significant, but the interaction was not significant.

72. Residual plots for ANOVA model of exterior surface roughness measurements, indicating no violations of assumptions of ANOVA.

73. Multiple comparisons for the four unique exterior surfaces measured average surface roughness.

74. Multiple comparisons test for electropolished and as-printed measured surface roughness for all surfaces. Electropolished is shown as '1' and as-printed as '2'.. 71

75. Laser polishing sample as-received (Left) front side of sample (Right) back side of sample.

76. 44x magnification SEM image showing two $4 \mathrm{~J} / \mathrm{cm}^{2}$ polishing squares contrasted with the bulk material.

77. SEM images of $4 \mathrm{~J} / \mathrm{cm}^{2}$ test squares showing ripples typical of re-solidification process immediately following laser polishing. (Left) 1258x image of bottommost polishing square containing significant cracking. (Right) 1240x image of second from the bottom polishing square showing powder particle warping ripple formation.

78. SEM images of border of $1 \mathrm{~J} / \mathrm{cm}^{2}$ test square showing the inability to deal with large parasitic powder particles; the un-polished metal is darker. 
79. (Left) 862x SEM image showing laser scan track intersection on front of part. (Right) 2262x SEM image of marked area on left image, showing large amounts of porosity and cracking of oxide layer present on surface.

80. (Left) 230x magnification SEM image of laser polishing on back of sample. (Right) 456x SEM image of parasitic powder particles with clear laser scan marks.

81. (Left) 1070x SEM image of potential heat-affected zone edge. (Right) 500x optical microscope image of potential heat affected zone microstructure. 77

82. SEM images of laser polished edge showing no sign of heat affected zone, but does show some porosity.

83. (Left) 200x, optical microscope image of microstructure. (Right) 551x SEM image of microstructure.

84. SEM images of etched laser polished sample, showing no significant change in microstructure as a result of laser polishing. 79

85. Laser profilometer measurement of as-received coupon. 80

86. Laser profilometer measurement of area exposed to $1 \mathrm{~J} / \mathrm{cm}^{2}$ energy density and 50 pulses

87. Laser profilometer measurement of area exposed to $1 \mathrm{~J} / \mathrm{cm}^{2}$ energy density and 175 pulses.

88. Laser profilometer measurement of area exposed to $4 \mathrm{~J} / \mathrm{cm}^{2}$ energy density and 75 pulses.

89. Laser profilometer measurement of area exposed to $4 \mathrm{~J} / \mathrm{cm}^{2}$ energy density and 125 pulses.

90. Laser profilometer measurement of area exposed to $4 \mathrm{~J} / \mathrm{cm}^{2}$ energy density and 175 pulses.

91. ANOVA model for the average measured surface roughness of the different scanned areas.

92. Four-in-one plot of residuals for the laser polishing average measured surface roughness models. 
93. Main effects plot for measured surface roughness of laser polished surfaces; direction 1 was measured across the scan path, while direction 2 was

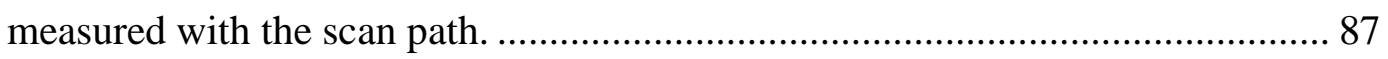

94. Dimensioned drawing of coin design. Dimensions in millimeters..................... 93

95. nTopology inputs and resulting lattice. The lattice unit cell is $3 \mathrm{~mm}^{3}$............... 94

96. Dimensions of larger coin sample. All dimensions in millimeters...................... 94

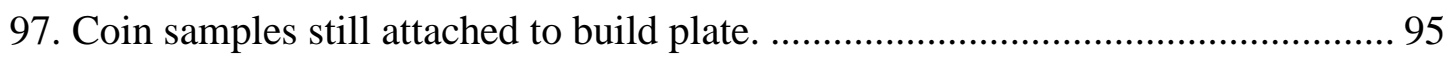

98. Large coin sample being weighed on the precision balance............................... 95

99. (Left) Small coin lattice immediately following extraction with a waterjet. (Right) The resulting lattices after being removed from their respective disks.... 96

99. Optical images of side view of larger lattice; significantly rough surface is clearly visible. 97

100. Small lattice after removal from disk, in position to be compression tested....... 98

101. Stress versus strain curve for large lattice samples........................................ 99

102. Stress versus strain for small lattice samples. 100

103. SEM images of large lattice sample prior to compression testing, demonstrating the poor surface condition of as-printed lattice. Strut diameter $=0.4 \mathrm{~mm}$. 103

104. SEM images of the large lattice sample post compression test. (Left) 69x, showing failed struts. (Right) 529x, showing gathering of parasitic particles on failed corner.

105. SEM images of failed small thin disk lattice structure. (Left) 61x, roughness of lattice is seen. (Right) 1276x, showing powder particles at the node a cell... 105 106. SEM images post electropolishing of (Left) small thin disk sample (Right) Large thin disk, both showing no polishing. 105

107. Unconstrained lattice samples showing some electropolishing, but still having issues with a good polish. 106 


\section{Introduction}

Additive manufacturing is the process of building three-dimensional parts by progressively adding thin layers of materials, as opposed to subtractive manufacturing, in which the three-dimensional parts are produced by removing material until the desired part size is achieved (DebRoy et al., 2018). A major benefit of this method is the ability to produce parts in their final, desired geometry, rather than employing multiple production steps, saving significant time and cost (Thomas, 2016). Additionally, complex geometries that may not have been possible with conventional production methods, such as internal features, are simple to produce. Parts are produced based on 3D rendered CAD drawings, exponentially expanding the amount of parts that can be produced, while fully eliminating the need to have molds for every part that needs to be made.

Additive manufacturing was initially developed as a rapid-prototyping process. However, as the technology has matured, it is now considered a direct replacement for some conventional processes in different industries (DebRoy et al., 2018). Additive manufacturing has potential applications in nearly every field, summarized in Table I. As can be seen on the table, stainless steel, titanium, and nickel super alloys have the most applications of additive manufacturing. As a result, these are the most widely studied alloys in literature. 
Table I: Common Additive Manufacturing Alloys and Applications (DebRoy et al., 2018)

\begin{tabular}{|l|c|c|c|c|c|c|}
\hline $\begin{array}{l}\text { Alloys } \\
\text { Applications }\end{array}$ & Aluminum & $\begin{array}{l}\text { Stainless } \\
\text { Steel }\end{array}$ & Titanium & $\begin{array}{l}\text { Cobalt } \\
\text { Chrome }\end{array}$ & $\begin{array}{l}\text { Nickel } \\
\text { Super } \\
\text { Alloy }\end{array}$ & $\begin{array}{l}\text { Precious } \\
\text { Metals }\end{array}$ \\
\hline Aerospace & $\mathrm{X}$ & $\mathrm{X}$ & $\mathrm{X}$ & $\mathrm{X}$ & $\mathrm{X}$ & \\
\hline Medical & & $\mathrm{X}$ & $\mathrm{X}$ & $\mathrm{X}$ & & $\mathrm{X}$ \\
\hline $\begin{array}{l}\text { Energy, oil, } \\
\text { and gas }\end{array}$ & & $\mathrm{X}$ & $\mathrm{X}$ & & & \\
\hline Automotive & $\mathrm{X}$ & $\mathrm{X}$ & $\mathrm{X}$ & & $\mathrm{X}$ & \\
\hline Marine & & $\mathrm{X}$ & $\mathrm{X}$ & & $\mathrm{X}$ & \\
\hline $\begin{array}{l}\text { Machinability } \\
\text { and } \\
\text { Weldability }\end{array}$ & $\mathrm{X}$ & $\mathrm{X}$ & $\mathrm{X}$ & & $\mathrm{X}$ & \\
\hline $\begin{array}{l}\text { Corrosion } \\
\text { Resistance }\end{array}$ & & $\mathrm{X}$ & $\mathrm{X}$ & $\mathrm{X}$ & $\mathrm{X}$ & \\
\hline $\begin{array}{l}\text { High } \\
\text { Temperature }\end{array}$ & & $\mathrm{X}$ & $\mathrm{X}$ & & & \\
\hline $\begin{array}{l}\text { Tools and } \\
\text { Molds }\end{array}$ & & $\mathrm{X}$ & & & & $\mathrm{X}$ \\
\hline $\begin{array}{l}\text { Consumer } \\
\text { Products }\end{array}$ & $\mathrm{X}$ & $\mathrm{X}$ & & & & \\
\hline
\end{tabular}

While a promising technology for exciting new applications, additive manufacturing will not replace many conventional manufacturing processes - rather, it will complement them and allow for unique new applications. Multiple obstacles still exist for the technology; size restrictions, process defects, production time, and cost. Additive manufacturing can only produce objects that fit inside the printer, meaning part size is limited by the printer. Printing multiple segments of parts and assembling them starts to deter from advantages of the additive manufacturing process. Additionally, the printing process is still relatively slow compared to conventional processes. Speed of printing would need to be increased to move additive manufacturing ahead of conventional manufacturing. The price of printers is still a barrier to entry as well (Attaran, 2017). Finally, there are inherent defects that occur during the printing process that require 
expensive and time-consuming post-processing. These defects will be discussed in detail in this review.

\subsection{Additive Manufacturing}

The additive manufacturing of metals originates from metal powder technology, welding, and prototyping. These technologies provide a good basis for additive manufacturing, but do not address many significant features of AM. The main similarities with welding are a moving heat source and "formation of a fusion zone with recirculating liquid metal that travels along with the heat source" (DebRoy et al., 2018). To increase the speed of production of this process, the scanning speed of the laser is often increased. However, this results in a longer molten pool, which can result in reduced properties (Mukherjee, Zuback, De, \& DebRoy, 2016).

ASTM Standard F2792 defines two categories of additive manufacturing: Directed Energy Deposition (DED) and Powder Bed Fusion (PBF). Figure 1 summarizes the key differences between these processes. 


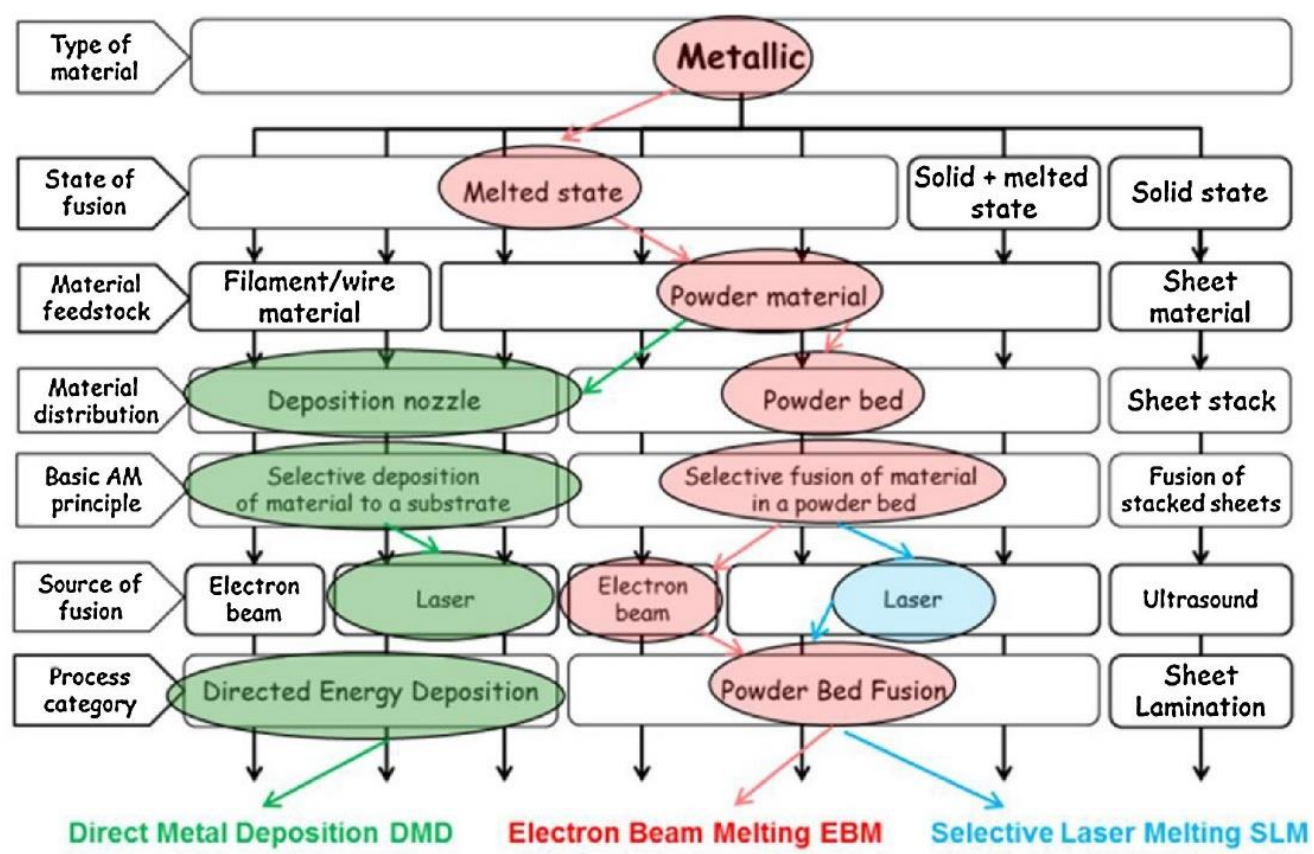

Figure 1: Differentiation of metal based AM processes (Schmidt et al., 2017).

Furthermore, these categories can be split up by function of primary heat source: electron beam (EB), laser (L), plasma arc (PA), and gas metal arc (GMA). This literature review will be focusing on PBF-L, also known as selective laser melting.

In PBF-L, the desired 3D part is modeled in CAD. The CAD file must be oriented to reduce the amount of support structures needed, as these must be removed after printing. The CAD model is then sliced into thin, planar layers. Next, a scan path must be defined. This is usually based on material and build specific parameters. The laser then scans the first layer into the powder bed. A thin layer of fresh powder is then spread across the surface by a raking, rolling, or combination of the two. Between each application of fresh powder, the Z-axis is moved incrementally downward (DebRoy et al., 2018). Fusion of the particles occurs by melting by the laser and the resulting solidification. An example of the machine setup can be seen in Figure 2. 


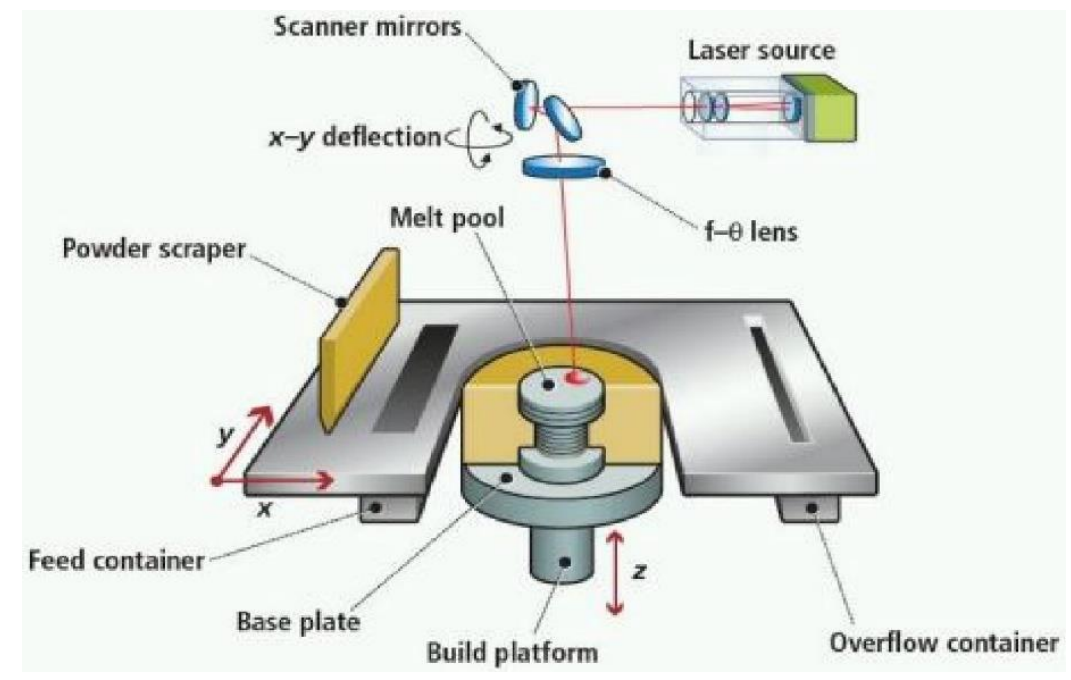

Figure 2: Summary of important components in the selective laser melting process (Schmidt et al., 2017).

As the laser beam hits the surface of the particles, the powder melts quickly into a molten pool. The highest temperature in this molten pool occurs directly below the heat source and decreases with increasing distance. Inside this pool, the Marangoni effect occurs, resulting in strong convective motion which produces a shear stress on the surface. This flow "mixes the liquid metal in different regions and enhances the transport of heat within the molten pool" (Mukherjee, Zhang, \& DebRoy, 2017). This has a strong effect on heating and cooling rates, solidification, and the evolution of various solid phases that produce the final microstructure (Mukherjee et al., 2017).

A large factor to be considered in PBF-L is the powder used. Particle size, shape, and distribution are key factors affecting the build. As stated earlier, some machines rake the fresh powder to spread, while others roll, and some do both. Machines that use a raking process require spherical powder, while machines that roll the powder do not require spherical geometry. Additionally, the atmosphere in both the storage unit and additive manufacturing machine can affect the surface condition of the powder, affecting the 
effective density, powder flow behavior, and chemical composition of the melt pool (Hebert, 2016). Materials like titanium and aluminum readily form oxide layers as well; careful consideration must be taken to prevent this. Powder that is not used can be partly recycled (Murr, Gaytan, Martinez, Medina, \& Wicker, 2012).

There are many opportunities for defects to occur during additive manufacturing. One defect that occurs is loss of alloying elements. Pronounced vaporization of alloying elements can take place when molten pool temperatures are high (DebRoy et al., 2018). Selective vaporization of more volatile elements can occur, which changes the overall composition of the alloy (Mukherjee et al., 2016). Three main defects will be discussed: residual stresses, porosity, and surface roughness.

\subsubsection{Residual Stresses}

Residual stresses are inherent to the additive manufacturing process. Due to the heating, melting, and solidification that happens as the laser scans across the powder in a layer by layer manner, different regions of the material experience differential heating and cooling (Mukherjee et al., 2017). The distribution of the thermal cycling across the workpiece results in residual stresses and distortion (Ding et al., 2011). If the magnitude of the residual stresses exceeds the yield strength of the material, cracking and delamination can occur (DebRoy et al., 2018). The residual stresses can affect corrosion resistance, fatigue strength, fracture toughness, and crack growth behavior (Mukherjee et al., 2017). There are two types of residual stresses: macro-stresses, which are on the scale of the dimensions of samples, and intergranular stresses, which are on the scale of the size of the grains (Wang et al., 2017). If the build plate is not pre-heated prior to deposition, the 
molten metal will try to shrink during solidification, but be constrained by the cold build plate, leading to in-plane tensile stresses. As more layers are deposited, the early layers are annealed, which relieves their tensile stresses. However, the solidification of the upper layers also produces compressive in-plane stresses in the early layers and build plate. The top-most deposited layers exhibit the highest tensile in-plane stresses due to lack of significant annealing and having their contraction constrained by a bulk of cold metal (Ghasri-Khouzani et al., 2017). Therefore, part height (number of powder layers) plays an important role in the magnitude of the residual stresses. (Mercelis \& Kruth, 2006) reported a four times increase of tensile in-plane stresses when increasing part size from $5 \mathrm{~mm}$ to $10 \mathrm{~mm}$. A finite element analysis model can be seen in Figure 3 .
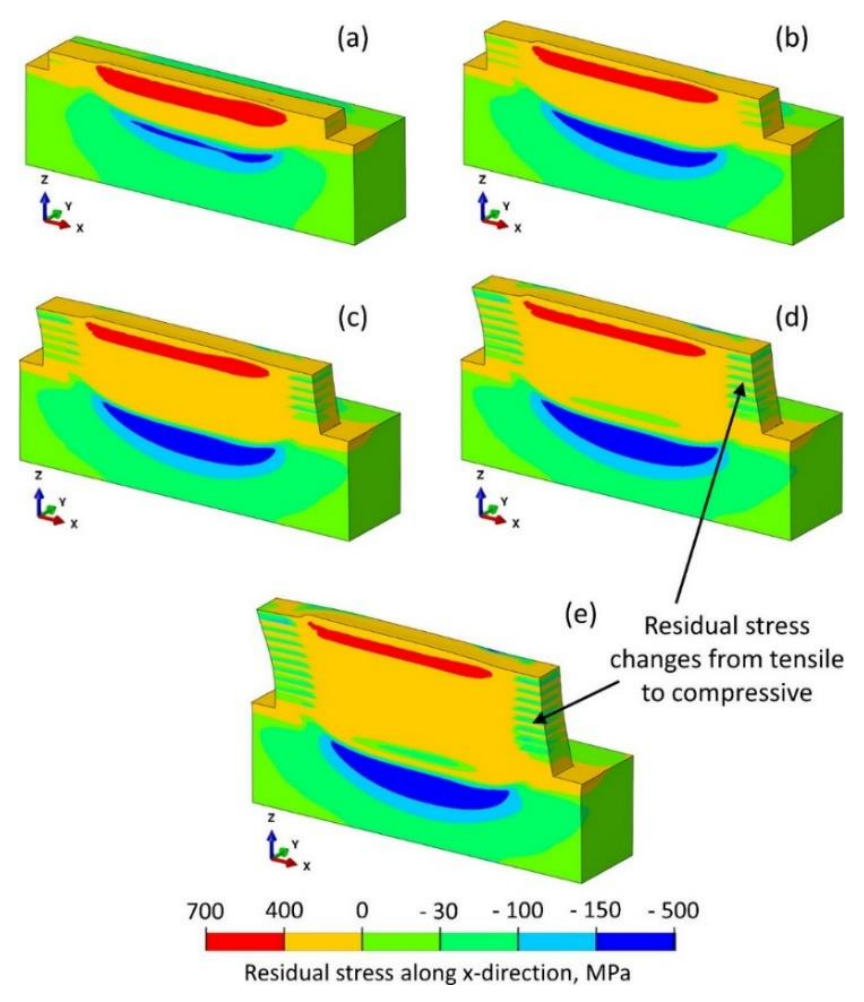

Figure 3: Residual stress along longitudinal direction at the end of deposition of (a) $2^{\text {nd }}$ (b) $4^{\text {th }}$ (c) $6^{\text {th }}$ (d) $8^{\text {th }}$ and (e) $10^{\text {th }}$ layer of IN 718 powder on IN 718 substrate (Mukherjee et al., 2017). 
In shorter parts, the thermal contraction is constrained by the base plate and is dominated by tensile stresses, but as height reaches a critical value, the thermal contraction of the upper layers is constrained by the already deposited material and stresses switch to compressive (Ghasri-Khouzani et al., 2017).

Multiple methods exist for measuring residual stress of parts, both destructive and nondestructive. Destructive testing includes hole drilling, which involves applying strain gauges to the surface of the sample and then drilling through holes and measuring the elastic deformation that occurs as the residual stresses are relieved. Also destructive, the contour method involves cutting the sample in half, measuring the elastic deformation, and computing the stresses using finite element analysis. Two non-destructive methods include neutron and x-ray diffraction (XRD), which are both based on Bragg's law of diffraction (Wang et al., 2017). Bragg's law of diffraction states that lattice spacing of specific sets of planes can be measured in stressed and stress-samples, and the lattice strain can be calculated from the difference. From the lattice strains, the residual strain can be calculated. Neutron diffraction is preferred in many applications, because XRD measurements are limited due to the depth of penetration of $\mathrm{x}$-rays $(\sim 5 \mu \mathrm{m}$ for steels), while neutrons can penetrate much deeper into metals ( $\sim 50 \mathrm{~mm}$ for steels) (GhasriKhouzani et al., 2017). However, neutron diffraction requires that the value of the stressfree lattice strain is precise, which can be difficult to know accurately due to a strong dependence on local chemical composition (DebRoy et al., 2018).

There are a multitude of build strategies that can be used to mitigate the amount of residual stresses produced. Decreasing the layer thickness can reduce the maximum longitudinal and through thickness residual stresses, however, it also increases the 
amount of distortion of the part (Mukherjee et al., 2017). Additionally, preheating the substrate, scanning in smaller islands, shorter deposition length, and increase in scanning speed were found to reduce or mitigate residual stresses. The preheating of the substrate was found to be the most effective strategy, as it reduces both the final residual stresses and the stresses during building. Change in scanning speed was found to be the least desirable, as it changes the geometry of the melt pool, which can produce undesirable changes in other properties of the fabricated part (DebRoy et al., 2018). Post-processing techniques to alleviate residual stresses include shot-peening, heat treatment, and most recently, ultrasonic nanocrystal surface modification (UNSM). UNSM is a form of ultrasonic shot-peening; both serve to reduce residual stresses by addition of beneficial subsurface compressive stresses. UNSM differs from shot-peening in the depth of applied compressive stresses (C. Ma et al., 2017). However, heat treatment is still the most common post-processing technique used.

\subsubsection{Porosity}

Porosity and voids are common defects in additive manufacturing (DebRoy et al., 2018). In general, porosity has a negative effect on mechanical properties of metals, especially fatigue strength, and should therefore be reduced (Tammas-Williams, Withers, Todd, \& Prangnell, 2016). Figure 4 depicts what porosity might look like in a cylinder immediately following printing. 


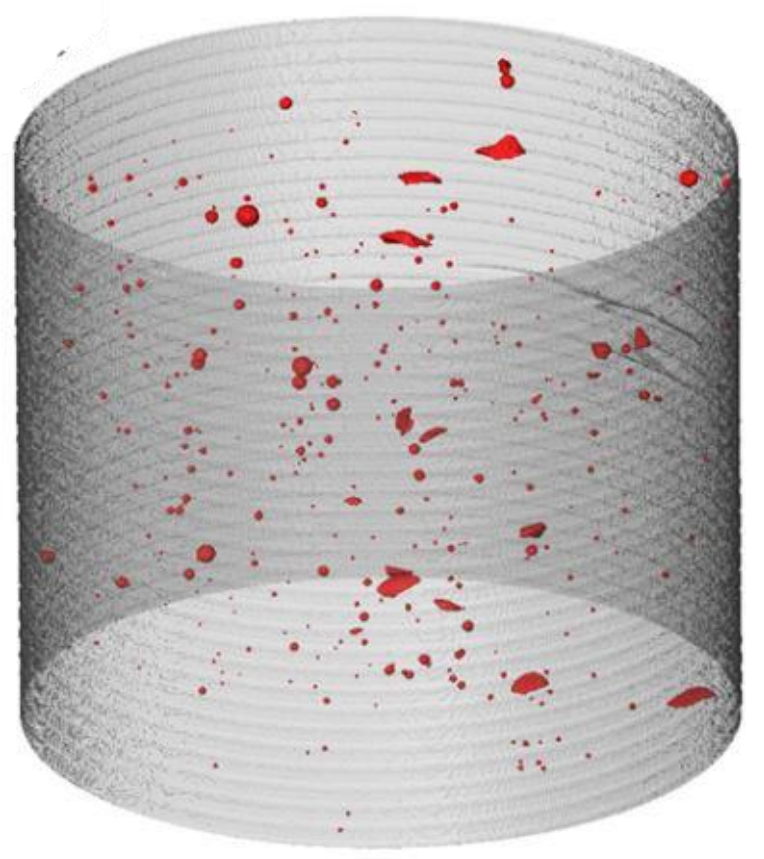

Figure 4: Visualization of porosity in selectively laser melted cylinder by x-ray microtomography (Tammas-Williams et al., 2016).

There are multiple causes for the porosity seen. When some selective laser melting processes are operated at a high power-density, melting of the powder can occur in keyhole mode. In keyhole mode, the power density of the laser beam is strong enough to cause evaporation, and the formation of plasma (King et al., 2014). The evaporation of the metal allows the laser to penetrate deeper than intended. If keyhole mode is not controlled, they can become unstable and collapse, leaving behind voids filled with vapor. A second cause of porosity is gas that becomes entrapped inside powder particles during powder processing. This can also be caused by shielding gas becoming entrapped in the molten pool. Finally, lack of fusion defects are caused by inadequate laser penetration of the upper layer (DebRoy et al., 2018).

Several methods exist for measuring porosity. The Archimedes method determines the density of the material, which can be used to determine the porosity (Slotwinski, 
Garboczi, \& Hebenstreit, 2014). The density is calculated as a ratio of the measured mass of the material in air and water. Porosity is then calculated as the difference between a theoretical fully-dense material and the calculated density. This method is simple and non-destructive, but gives no information about size, distribution, or geometry of pores, and can be affected by surface cracks and pores that allow water infiltration (Slotwinski et al., 2014). Optical microscopy, scanning electron microscopy (SEM), x-ray computer tomography (x-ray CT), and synchrotron radiation micro-tomography $(\mathrm{SR} \mu \mathrm{T})$ are additional methods to measure porosity. Optical microscopy and SEM are destructive measurement methods, with optical microscopy being less useful than SEM due to its inability to detect pores smaller than $50 \mu \mathrm{m} . \mathrm{X}$-ray $\mathrm{CT}$ and $\mathrm{SR} \mu \mathrm{T}$ are both capable of insitu measurements, and can accurately measure size, distribution, and geometry of pores. Hot isostatic pressing is often used to close internal pores, but struggles with surface cracks (DebRoy et al., 2018).

\subsubsection{Surface Roughness}

Factors contributing to surface quality of additively manufactured parts include alloy type, powder shape, size, and morphology (DebRoy et al., 2018). There are also two main mechanisms by which surface roughness occur. One of the main mechanisms producing surface roughness is the "stair step" effect, which is due to the layer-by-layer approximation of sloped and curved surfaces (Strano, Hao, Everson, \& Evans, 2013). Figure 5 shows a representation of this effect. 


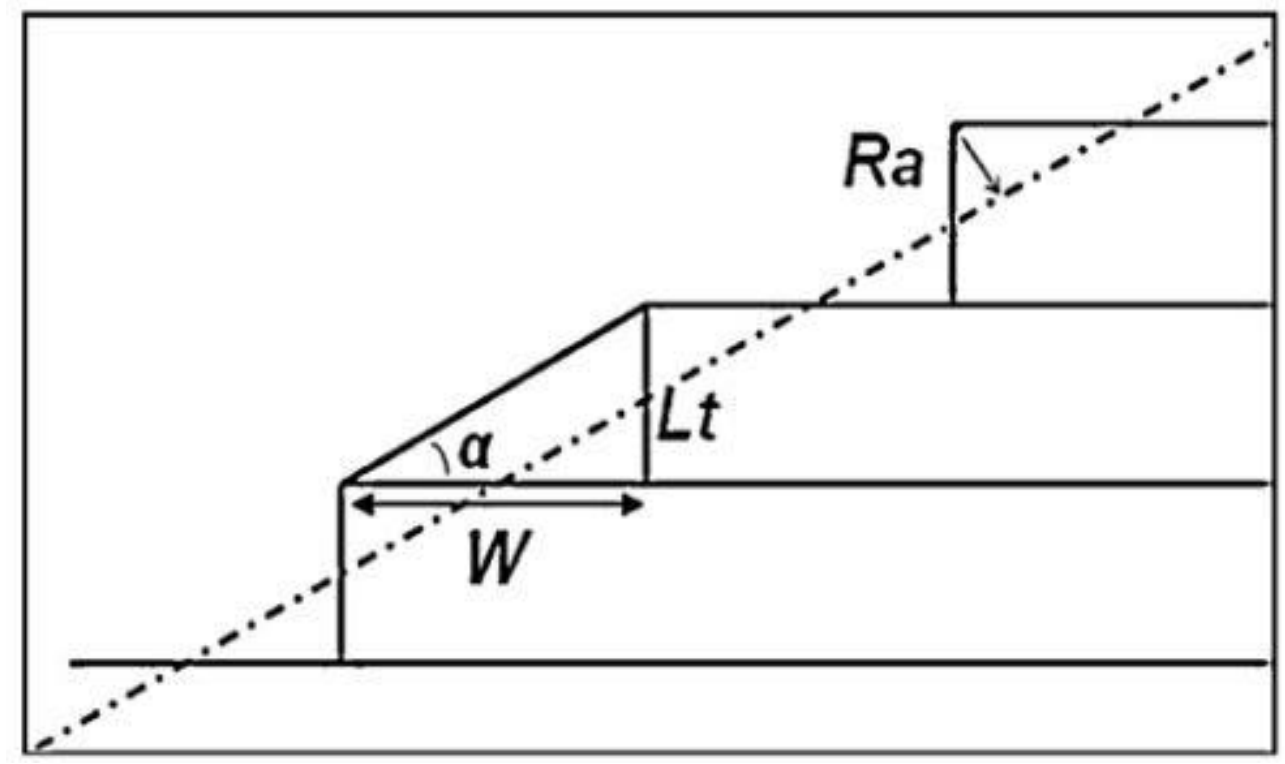

Figure 5: Schematic of surface error caused by stair step effect on an inclined surface (Strano et al., 2013).

This effect is present in all layer-by-layer additive manufacturing processes that include sloped or curved surfaces. The effect is much more pronounced in unsupported overhanging faces as well, as un-melted powder supports the faces, instead of solid material. Instead of fusing with solid material, the melt pool of the unsupported features sinks into the powder by gravity and capillary action (Urlea \& Brailovski, 2017). The stair step effect can be mitigated by reducing layer thickness to better approximate the surfaces; however, this causes a sharp increase in build time, and is limited by available powder size, and is therefore not necessarily a viable solution (DebRoy et al., 2018). A simpler strategy is to select the proper build orientation to avoid a sharp build orientation and reduce the number of unsupported overhangs.

The second mechanism producing surface roughness the balling phenomenon. Balling phenomenon can result in the formation of discontinuous scan tracks, and can impede the uniform deposition of fresh powder, leading to porosity. Balling is dependent on powder 
material properties, but can be controlled with laser processing conditions (Gu \& Shen, 2009). There are two primary methods by which balling phenomenon occurs; low laser power, and high scan speed. Figure 6 depicts the first type of balling phenomenon, in which coarsened balls are formed by limited liquid formation.

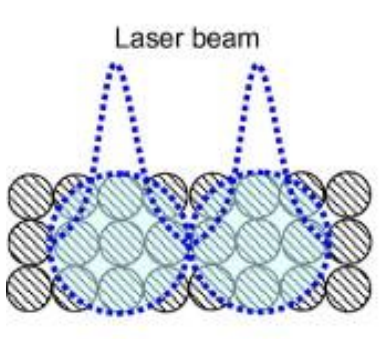

Powder bed

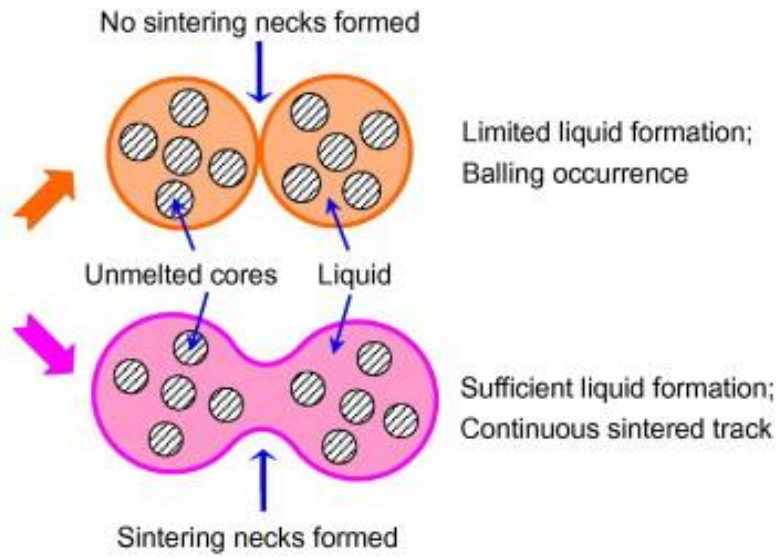

Figure 6: First kind of balling phenomenon caused by limited liquid formation ( $\mathrm{Gu} \&$ Shen, 2009).

The amount of liquid formation is dependent on the operating temperature of the sintering system, which is controlled by laser power and scan speed. For a given scan speed and lowering laser power, the overall rheological performance of the liquid decreases. This causes the molten materials to "aggregate into an individual coarsened sphere approximately the diameter of the laser beam" (Gu \& Shen, 2009). Figure 7 is an SEM image of the schematic in Figure 6. 

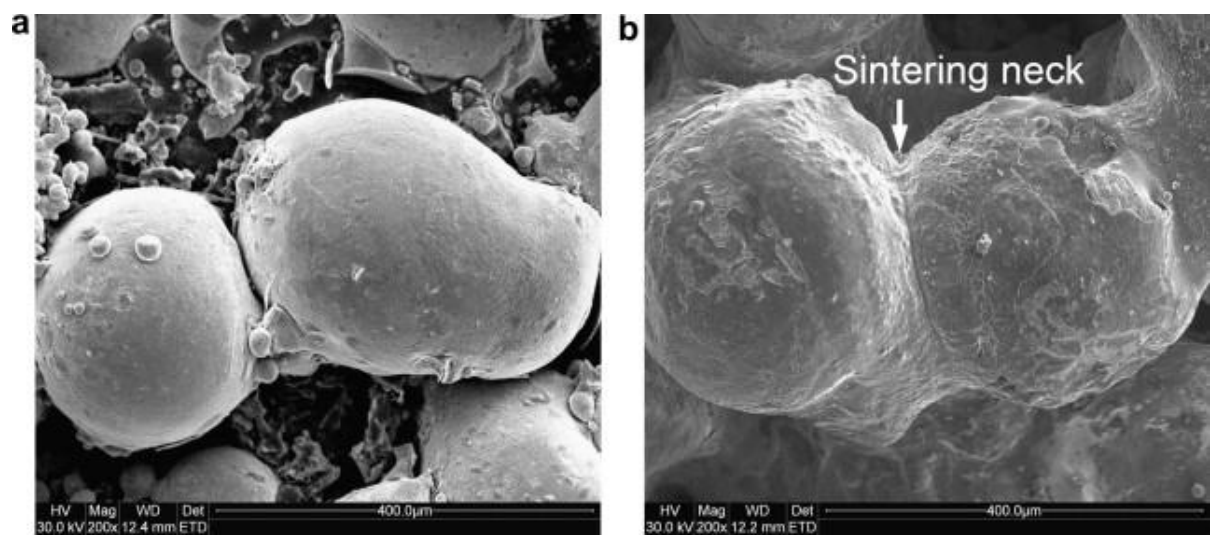

Figure 7: SEM images of 316L SS balling phenomenon (a) with no sintering neck (b) with sintering neck (Gu \& Shen, 2009).

Both images feature insufficiently melted particles, which produce surface roughness.

The second phenomenon is due to too high scan at sufficient laser power. Figure 8 shows how the molten pool changes in geometry as the scan speed is increased.
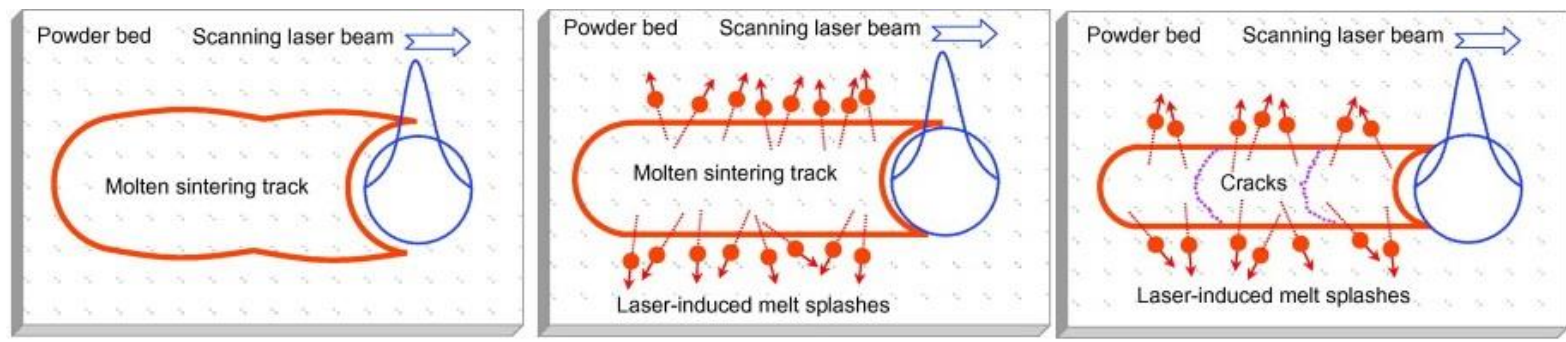

Increasing laser scan speed

Figure 8: Depiction of change in molten pool geometry and size as laser scan speed is increased at given laser power (Gu \& Shen, 2009).

As the scan speed is increased, a decrease in the working temperature is seen, which increases the diameter of the cylindrical molten track. This produces a melt instability, which causes a number of "small-sized liquid droplets" to splash from the surface of the molten track (Gu \& Shen, 2009). Figure 9 depicts SEM images of the second balling phenomenon. 

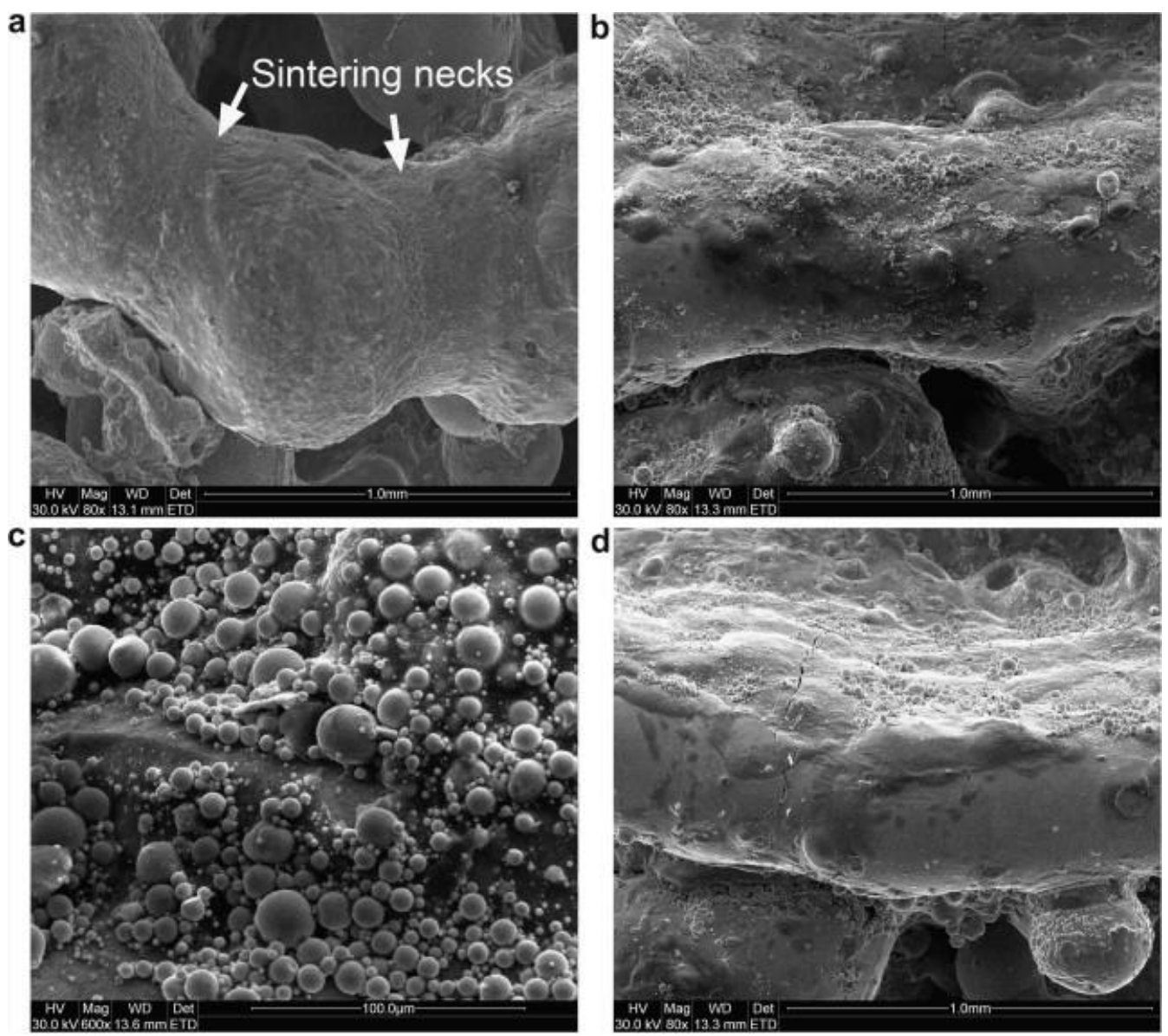

Figure 9: SEM characterization of 316L SS second balling phenomenon at different scan speeds (a) shows similar formation to first balling phenomenon (b) is a higher speed (c) is a magnification of (b) (d) is an even higher scan rate (Gu \& Shen, 2009).

The laser speeds used in Figure 9 are high enough to produce the second balling phenomenon; however, if they were increased, the shape of the molten pool could be altered even further and result in longitudinal cracks after solidification (Gu \& Shen, 2009). To control the two balling phenomenon, a sufficient laser power at appropriate scan speed must be selected. This is estimated by a parameter known as the linear energy density, which is a ratio of the laser power to the scan speed. 
In addition to balling and the stair-step effect, there exists two other causes of surface roughness. The first is known as the ripple effect, which is most often observed on horizontal surfaces. As previously discussed, shear forces exist on the surface of the melt pool due to the thermal gradient present. During cooling, as the thermal gradient is reduced, surface tension attempts to level the surface. However, due to the viscosity of the liquid, the surface cannot be fully leveled before cooling is finished (Urlea \& Brailovski, 2017). The final main cause of surface roughness is due to parasitic particles. As the laser reaches the edge of each layer, the laser power must be reduced to avoid unwanted melting of the powder. However, there is still enough thermal energy to sinter the particles to the surface of the workpiece without being melted. Similar to the stair step effect, unsupported overhangs often end up with the highest number of particles attached to their surfaces.

Besides a proper scanning strategy, many post-processing techniques such as mechanical polishing, magnetic field assisted finishing, and peening exist to improve surface roughness. These will be discussed in further detail in Section 1.2.

\subsection{Surface Treatments}

Surface finish is important to control, as it affects biological response, mechanical properties like fatigue strength, and heat transfer properties. As previously mentioned, many surface roughness treatments exist. Mechanical treatments for surface roughness include milling, abrasive blasting, vibratory grinding, and micro machining (Bagehorn, Wehr, \& Maier, 2017). The authors of the study found milling to produce the best surface roughness, but micro machining and vibratory grinding were not far behind. However, 
most mechanical treatments are limited to external features, and need to be used carefully on thin-walled features (Mohammadian, Turenne, \& Brailovski, 2018). As one of the most attractive features of additive manufacturing is the ability to produce internal geometries, it is important to develop techniques that can improve interior surface roughness. Non-mechanical methods that have been investigated include chemical polishing, electrochemical polishing, and abrasive flow machining. Abrasive flow machining is limited in the size of the internal cavities it can polish due to the high viscosity of the matrix (Mohammadian et al., 2018). Chemical polishing works well, but is limited by the alloy selection; many of the most desirable alloys require high strength acids, such as hydrofluoric, which are dangerous and hard to work with (Urlea \& Brailovski, 2017).

\subsubsection{Laser Polishing}

Laser polishing is an attractive alternative to mechanical polishing processes, as it can be fully automated, and has no tool force, therefore allowing use in applications in which geometric tolerance is critical. Three different mechanisms exist to polish using laser irradiation, which are large area ablation, localized ablation, and re-melting at macro or micro polishing regimes (Bhaduri et al., 2017). Figure 10 shows a visual comparison of the three methods. 


\section{Cross-section of initial profile}

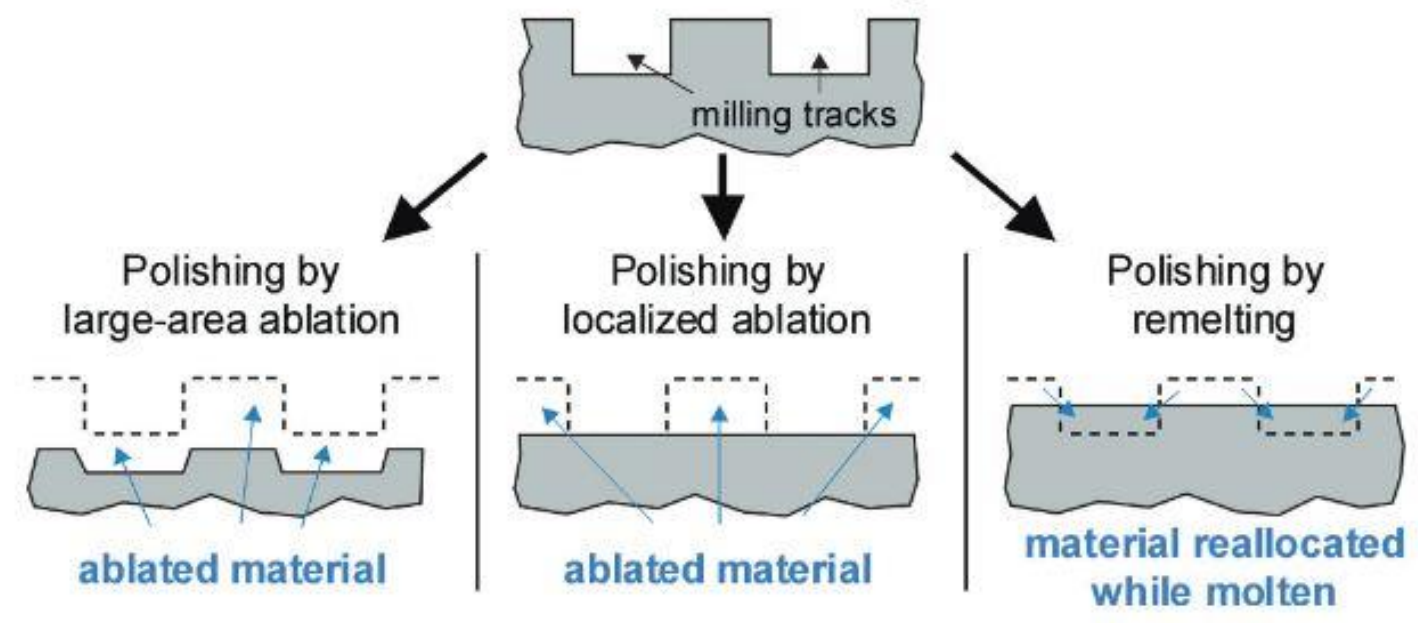

Figure 10: Visual Comparison of three different laser polishing processes (Willenborg, 2011).

A majority of laser polishing research has focused on the re-melting process due to several advantages over ablation methods: high level of automation, short machining times, no pollutive impact from grinding or chemical wastes, user definable surface roughness, no changing the form of the workpiece, and a small micro roughness finish (Willenborg, 2011).

Macro polishing is done using continuous wave laser radiation. Generally, fiber-coupled neodymium-doped yttrium aluminum garnet (Nd:YAG) are used with laser powers of 70$300 \mathrm{~W}$. This process can be used on processed surfaces with a roughness $R a$ up to several micrometers, such as milled, turned, or electric discharge machined surfaces. Variables affecting the achievable surface roughness of macro polishing are initial surface roughness and lateral dimensions of the surface, thermo-physical properties of the material, homogeneity of the material, and grain size (Willenborg, 2011). Surface tension attempts to produce a perfectly smooth surface, but during the re-melting and solidification, remains of initial roughness, ripples, undercuts, step structures, bulges, and 
martensite needles prevent this (Willenborg, 2011). Figure 11 is a schematic of these effects.
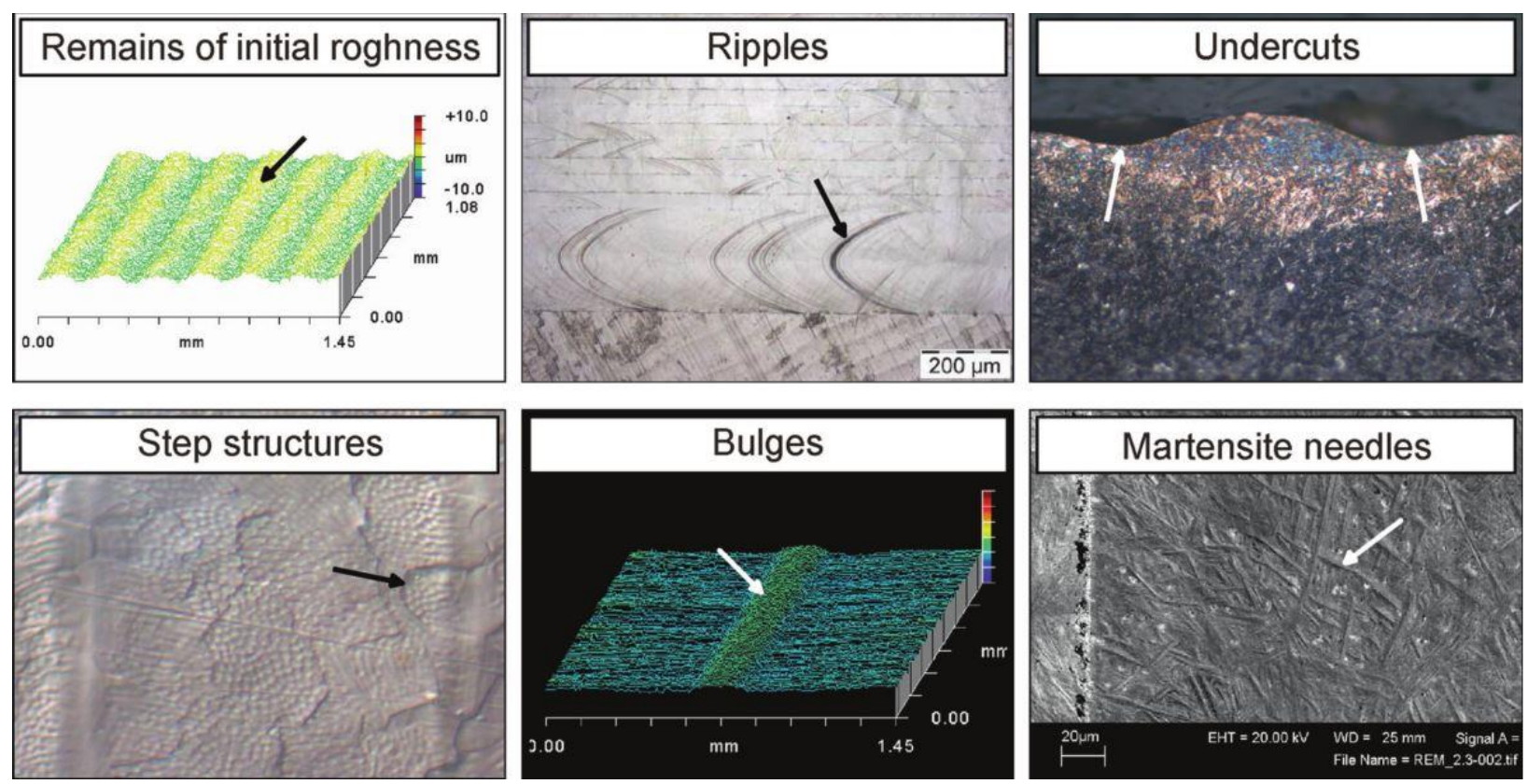

Figure 11: The six main effects preventing surface tension from producing a perfectly smooth surface (Willenborg, 2011).

All of these effects need to be considered when performing laser polishing, as they are all present in the selective laser melting technology.

On the other hand, micro polishing is performed with pulsed laser radiation, with the pulse duration in the range of $20-1000 \mathrm{~ns}$, and a re-melting depth of $0.5-5 \mu \mathrm{m}$ (Willenborg, 2011). Micro polishing requires a fine surface before processing, however, it is able to produce an even finer surface than macro-polishing. Figure 12 depicts the reduction in surface roughness achievable on a flat surface. 


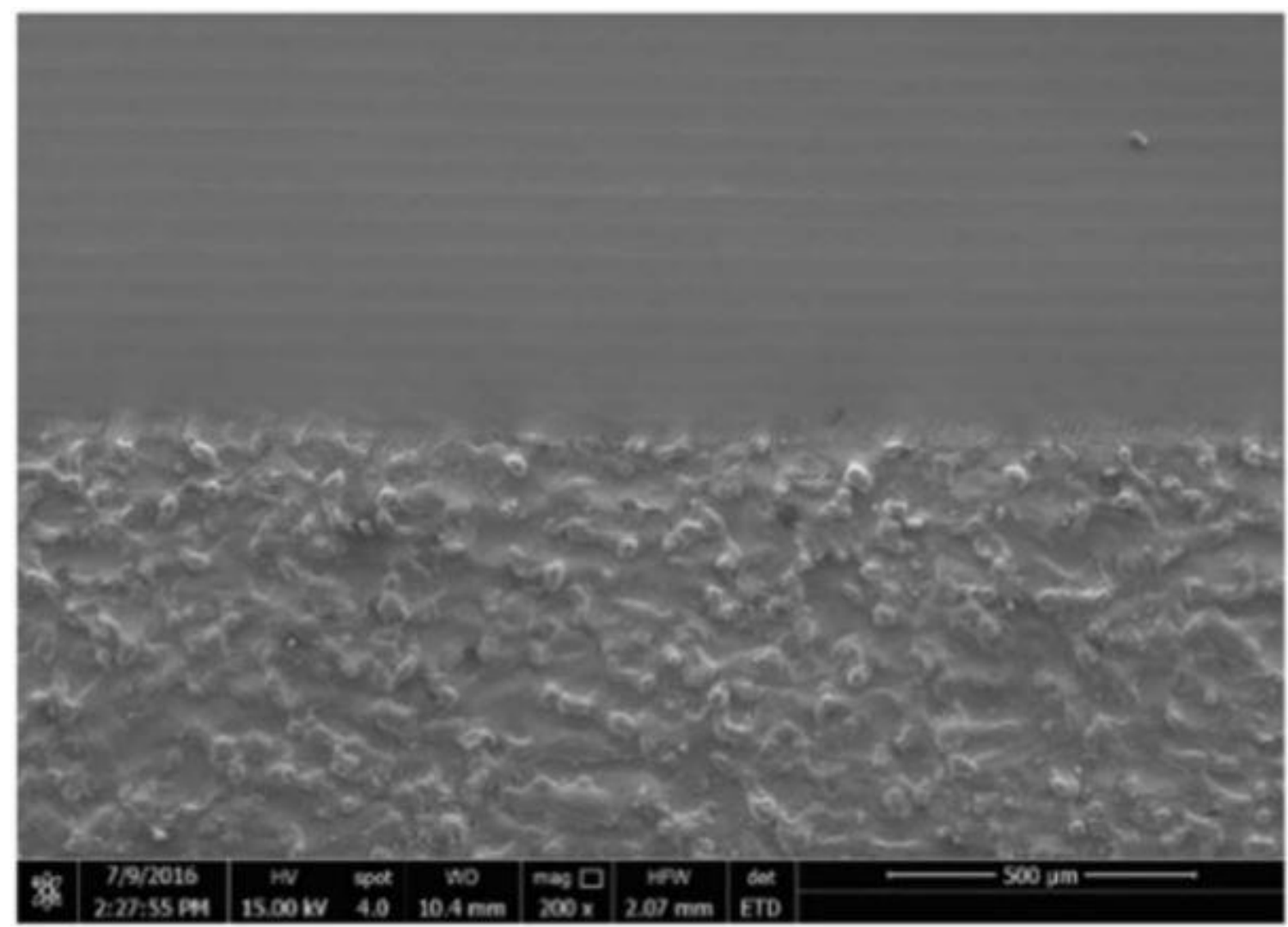

Figure 12: The boundary between as selectively laser melted and laser micro polished TC4 Ti alloy (C. P. Ma, Guan, \& Zhou, 2017).

While track marks can be seen where the laser polishing occurred, the surface roughness is significantly reduced. However, the small re-melting depth cannot eliminate large surface structures. Key process parameters are pulse duration and intensity (Willenborg, 2011). Due to the speed of the re-melting occurring at the surface, the microhardness is often increased as well (De Giorgi et al., 2016). The depth of metal affected by the laser polishing process can be up to $100 \mu \mathrm{m}$, shown in Figure 13. 


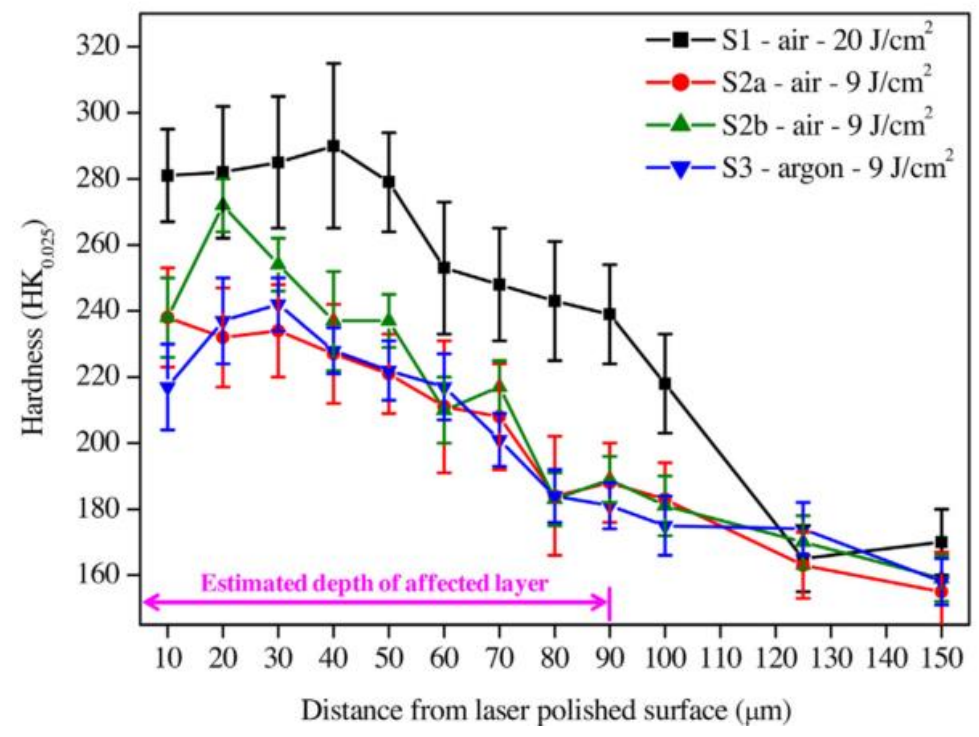

Figure 13: Hardness measurements of laser polished 316L at different energy densities and polishing environments (Bhaduri et al., 2017).

The higher the energy density, the harder the surface produced, but the amount of material affected is approximately the same. Additionally, depending on the environment that the polishing occurs in, the heat affected zone can contain more impurities and porosity, seen in Figure 14.
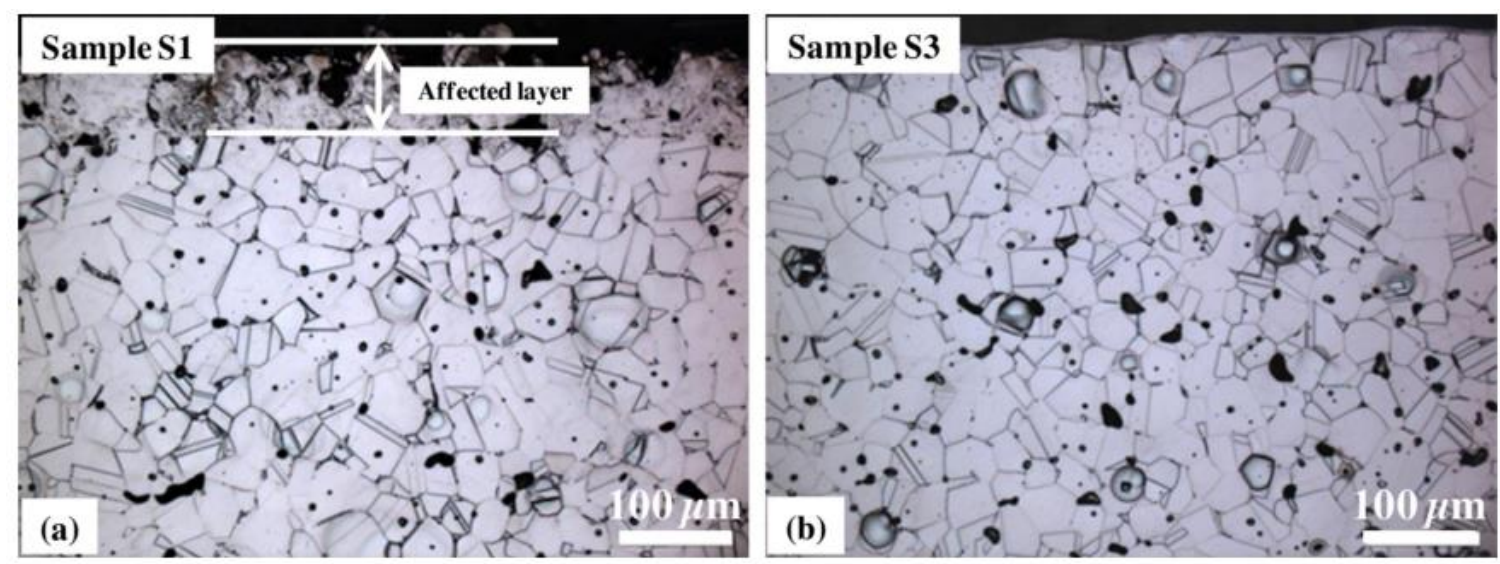

Figure 14: Microstructure of etched 316L following laser polishing at (a) $20 \mathrm{~J} / \mathrm{cm}^{2}$ in air and (b) $9 \mathrm{~J} / \mathrm{cm}^{2}$ in argon (Bhaduri et al., 2017). 
The sub-surface of the left sample contains oxides, lumps, holes, pits, and irregularities, while the sample on the right has no sign of oxide layers. Additionally, there is no distinguishable grain structure difference, even up to the assumed affected area (Bhaduri et al., 2017). To achieve laser polishing, fiber-coupled Nd:YAG lasers are used, as well as excimer lasers. Table II summarizes the key parameters of micro and macro polishing.

Table II: Laser Polishing Key Parameters (Willenborg, 2011)

\begin{tabular}{|c|c|c|c|c|c|c|c|}
\hline \multirow[t]{2}{*}{ Process } & Initial & Final & Laser & Pulse & Processing & Power & $\operatorname{Re}-$ \\
\hline & $\boldsymbol{R} a$ & $R \boldsymbol{a}$ & Type & Duration & Time & & melting \\
\hline
\end{tabular}

\begin{tabular}{c|c|c|c|c|c|c|c|}
\hline Macro & $3-10$ & $0.5-1$ & Fiber & Continuous & $10-200$ & $70-$ & $20-200$ \\
Polishing & $\mu \mathrm{m}$ & $\mu \mathrm{m}$ & $\mathrm{Nd}$ :YAG & & $\mathrm{s} / \mathrm{cm}^{2}$ & $300 \mathrm{~W}$ & $\mu \mathrm{m}$ \\
\cline { 2 - 7 } Micro & $\begin{array}{c}0.3- \\
\text { Polishing }\end{array}$ & 0.1 & Fiber & $20-1000 \mathrm{~ns}$ & $<3 \mathrm{~s}^{-\mathrm{cm}^{2}}$ & $70-$ & $0.5-5$ \\
& & $\mu \mathrm{m}$ & Nd:YAG, \\
& & & & $300 \mathrm{~W}$ & $\mu \mathrm{m}$ \\
\hline
\end{tabular}

Because the micro polishing process is limited to surfaces that are already smooth, macro and micro polishing are often performed sequentially; macro polishing removes the larger surface structures, while micro polishing removes the final roughness. Even though laser polishing cannot affect interior surfaces, the redistribution of surface asperities to fill in valleys makes it an attractive process, as there is little-to-no mass loss in the polishing process.

\subsubsection{Electropolishing}


The most attractive feature of electropolishing is in its ease of polishing internal cavities when compared to other polishing processes. Electropolishing is a form of electrochemical machining; it allows manufacturers to shape any conductive material with no tool force. To electropolish, an electric circuit must be formed between a tool and workpiece connected to an external electric source. They are then submerged in an electrolyte bath to close the circuit. Material removal occurs by a localized anodic dissolution at the workpiece. This is produced by the deliberate application of an external potential difference, known as the overpotential. The overpotential must be high enough to maintain a steady ion migration, otherwise a non-uniform surface finish will be attained, however, it cannot be too high either. Another important parameter in electrochemical polishing is the current density, which is the measure of electric current that flows per unit area. Like overpotential, current density must be sufficiently high to maintain the proper dissolution of metal, but it must not be too high. The interplay between current density and overpotential plays a deciding factor in the type of surface finish produced by electrochemical polishing, as seen by (Gomez-Gallegos, Mill, \& Mount, 2016), who performed electrochemical polishing of 316 stainless steel pipes. Their results can be seen in Figure 15. 


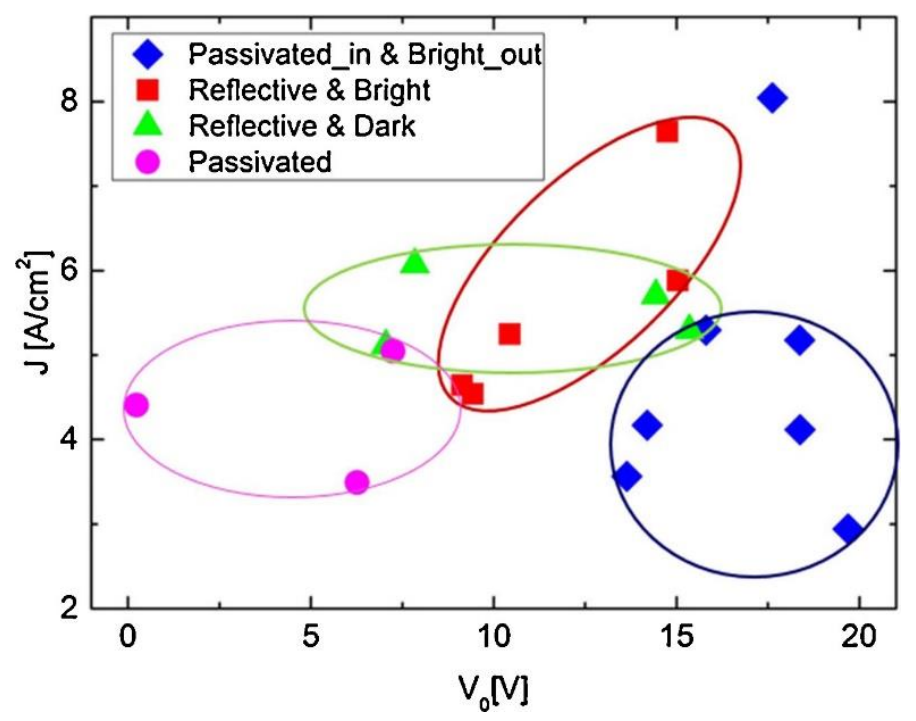

Figure 15: Different surface finishes of 316 SS as a function of current density and overpotential (Gomez-Gallegos et al., 2016).

The rhomboids in Figure 15 represent areas where a non-uniform surface finish was achieved. These regions are also referred to as being below the oxygen evolution potential, and at it. Below the oxygen evolution potential, the metal is dissolved and passivated at the same time. At the oxygen evolution potential, the electropolishing is "carried out during the dissociation of the aqueous electrolyte" (ur Rahman, Deen, Cano, \& Haider, 2017).

Another important process parameter is the flow rate of the electrolyte. It serves two main purposes: removal of metal ions, and temperature control of the system. A change in temperature can affect the conductivity of the electrolyte. An important feature of electrochemical polishing is the difference in dissolution rates between the peaks and valleys of rough surfaces. If the dissolution rate was similar, no polishing would occur; however, peaks are preferentially dissolved, leading to a reduction in roughness as the polishing process continues (Habibzadeh, Li, Shum-Tim, Davis, \& Omanovic, 2014). 
Electropolishing of multiple alloys produced by additive manufacturing has been investigated by researchers. For 316L SS, a study was able to reduce surface roughness to $103 \mathrm{~nm}$ (Rotty, Mandroyan, Doche, \& Hihn, 2016). In a study that investigated electrochemical polishing of Inconel 718, researchers found a slight decrease in surface roughness, but more importantly, found that a short electrochemical polishing was enough to remove parasitic particles from the surface of their parts (Baicheng et al., 2017). Other researchers investigated the electrochemical polishing of pure titanium produced by electron beam melting and found that it is possible to completely flatten the surface, which can be seen in Figure 16.
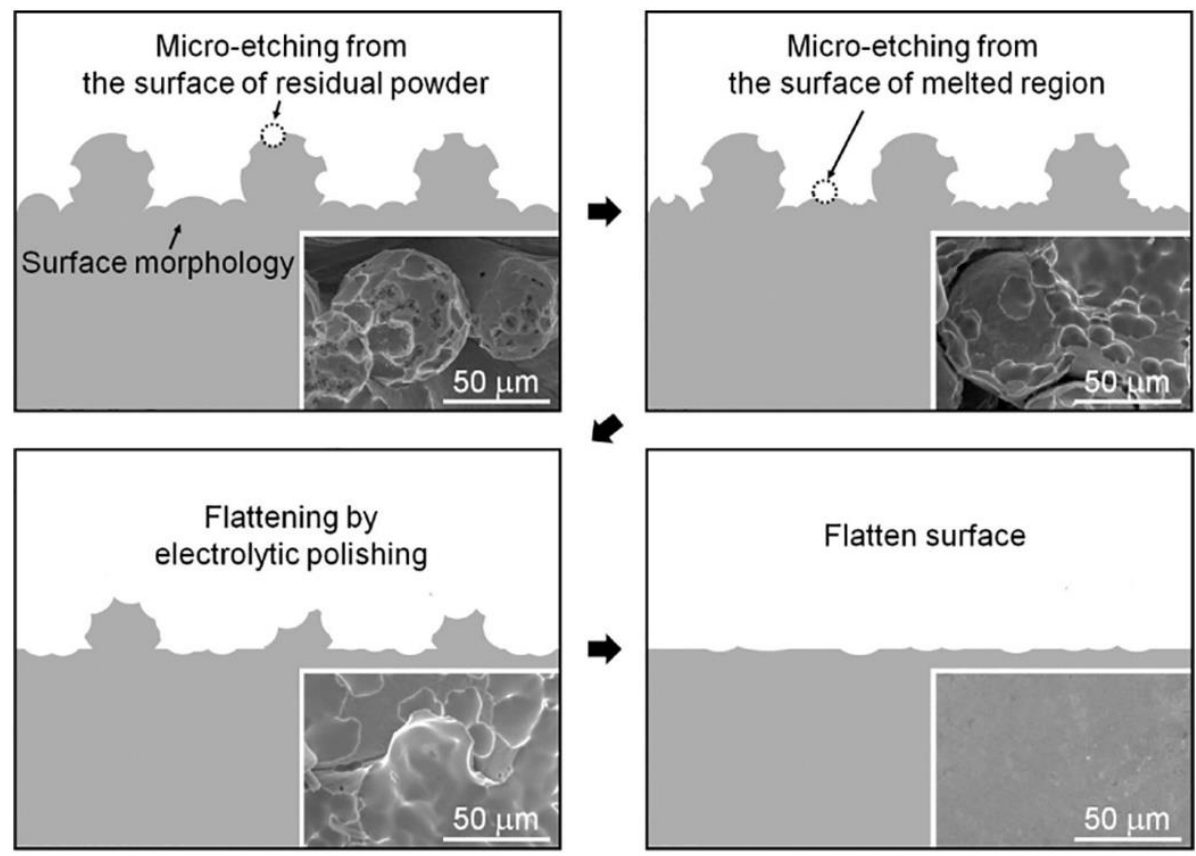

Figure 16: Schematic showing the flattening process of electrochemical processing of pure titanium (Jung et al., 2017).

It can be seen that the electrochemical polishing process etches the un-melted powder preferentially, which allows for polishing in steps. This also allows for short polishing step to remove only parasitic powder particles. However, the researchers reported a 
significant loss in mass when bringing the process to the maximum reduction in surface roughness, which could prove problematic for the technology in applications that require tight tolerances and cannot afford to lose significant mass (Jung et al., 2017).

\subsection{Solidification Structures and other Material Properties}

As discussed in the Technology section, selective laser melting occurs via a scanning laser melting a powder bed in a layer-by-layer process. The powder absorbs energy from the laser through both powder-coupling and bulk-coupling mechanisms (Fischer et al., 2003), and the remaining energy is distributed to the underlying layers. This process is known as subsequent thermal cycling (STC), which causes many thermally activated diffusion processes like grain growth and precipitation, leading to unique microstructures in PBF-L not seen in conventionally machined alloys (Liu, Zhang, \& Pang, 2018). STC is highly dependent on processing parameters, such as laser power, scan speed, thickness of powder layer, and bulk material properties. Understanding the thermal interactions between successive layers is important to STC, as the change of the material from powder to solid, as well as the size of the thermal gradient, affects the heat transfer mechanisms, and consequently, the thermally activated diffusion processes (Shi, Gu, Xia, Cao, \& Rong, 2016). In comparison to casting processes, fine-grained microstructures are usually seen in additive manufacturing processes. When compared to other metal-based additive processes, selective laser melting generally produces the finest grain size due to the smaller melt pool size (Herzog, Seyda, Wycisk, \& Emmelmann, 2016). An especially important consideration is the cooling rate experienced by the powder, which can be 
orders of magnitude faster than quenching, which can often result in metastable microstructures. The effect of undercooling can be seen in Figure 17.
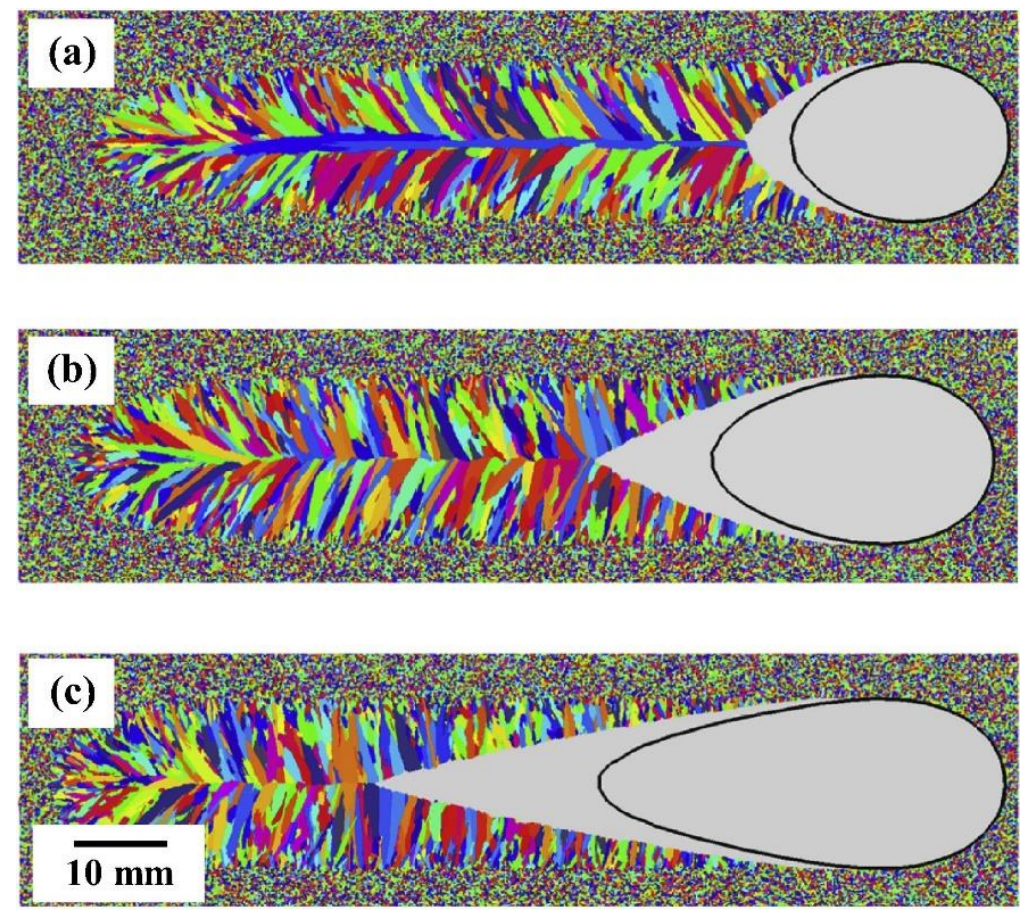

Figure 17: Grain structure produced as a function of undercooling due to increasing scan speed (DebRoy et al., 2018).

Beyond the difference seen in grain orientation, actual solidification occurs further away from the melt pool depending on scanning speed. Additionally, the layer-by-layer deposition method results in a vertically graded microstructure, which is further exacerbated by STC (Gussone et al., 2017). Figure 18 depicts this vertically graded microstructure. 


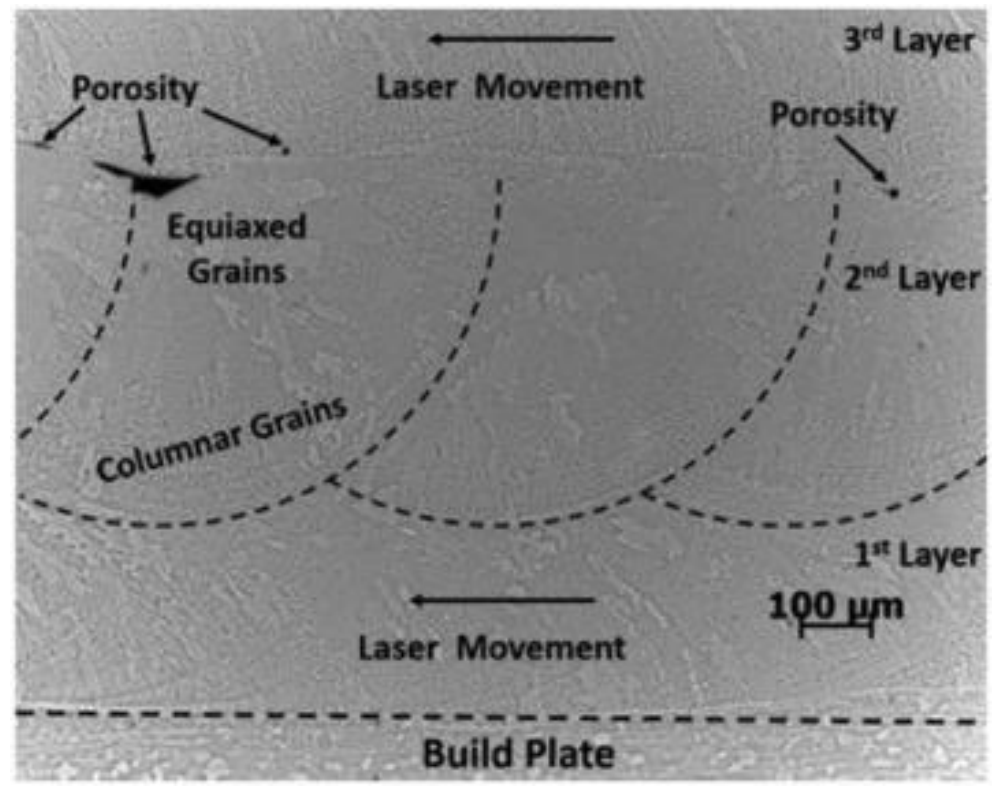

Figure 18: Three layers of additively manufactured 316L showing microstructural features (Herzog et al., 2016)

Mechanical properties are based on many factors of the SLM process as well. In a study on the effect of layer thickness in Inconel 718, it was found that samples produced with a smaller layer thickness were stronger and less ductile. Additionally, the samples tested horizontally to the build direction were stronger and less ductile than vertical samples. This is attributed to the columnar dendritic cell microstructure that is characteristic of the SLM process (Sufiiarov et al., 2017). The factors leading to columnar dendritic microstructures is shown in Figure 19. 


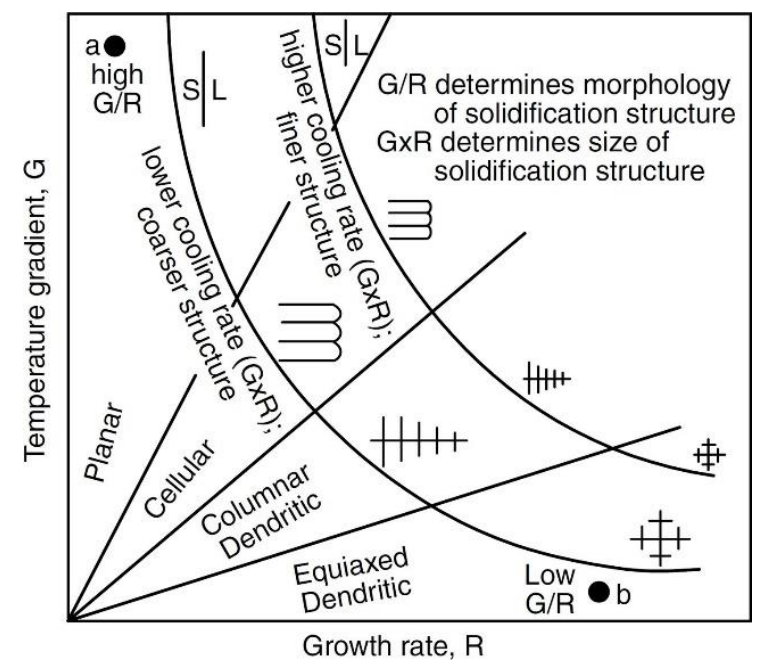

Figure 19: Thermal gradient versus cooling rate and its effect on grain morphology and size (DebRoy et al., 2018).

The columnar dendritic microstructure is a result of the increase in capillarity in the SLM process, which increases the crystal growth trend (Khorasani, Gibson, Goldberg, \& Littlefair, 2016). These columnar structures occur when thermal gradient aligned dendrites outgrow misaligned dendrites (Wei, Elmer, \& DebRoy, 2017). Grain morphology, size, and texture that result from solidification are controlled by the thermal conditions that exist prior to the start of solidification, while the fine-scale microstructure is controlled by post-solidification cooling rate (Farshidianfar, Khajepour, \& Gerlich, 2016). Nucleation is not a key factor during selective laser melting due to unchanging chemical composition throughout the process. Therefore, grains grow epitaxially and are oriented towards the molten pool (Thijs, Verhaeghe, Craeghs, Humbeeck, \& Kruth, 2010). 


\subsection{Lattices}

Due to their ability to provide large superior specific stiffness and strength, low thermal conductivity, and high toughness, lattices, or micro-cellular structures, have been investigated using many methods of production (Recep Gümrük, Mines, \& Karadeniz, 2013). Due to its high complexity, powder-bed fusion with a laser is an excellent contender for producing these lattice structures. Additionally, these structures require considerably less material to produce, leading to cost savings. A few sample unit cells can be seen in Figure 20.

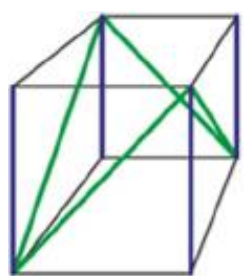

fcc,, $\mathrm{Z}$

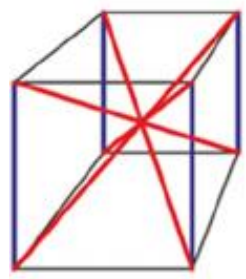

bcc, $z$

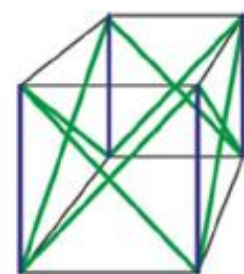

$\mathrm{f}_{2} \mathrm{Cc}, \mathrm{Z}$

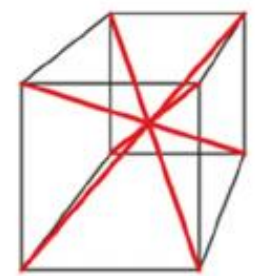

bcc

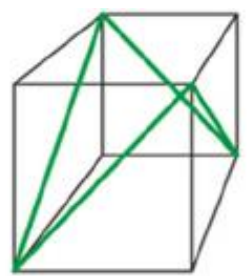

fcc

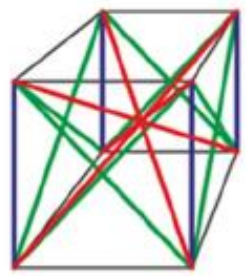

$\mathrm{f}_{2} \mathrm{bcc}, \mathrm{z}$

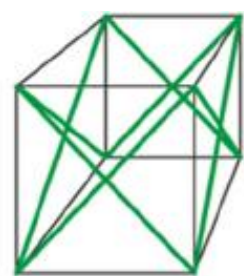

$\mathrm{f}_{2} \mathrm{CC}$

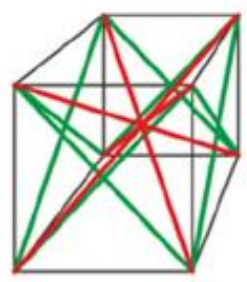

$\mathrm{f}_{2} \mathrm{bcc}$

Figure 20: A handful of different unit cell types used for lattice structures (Merkt, Hinke, Bültmann, Brandt, \& Xie, 2015).

An important concept in analyzing the behavior of the lattice structure is to categorize between stretch and bending-dominated. A stretch dominated structure is "exceptionally stiff and strong relative to its weight” (Brøtan, Fergani, Sørby, \& Welo, 2016). These structures are generally filled with triangles, due to their structural stability without any bending forces. A bending-dominated structure may not be as stiff or strong, but will 
absorb energy when placed under compressive forces (Brøtan et al., 2016). Generally, bending-dominated structures are constructed from polygons with more than three angles, resulting in a more flexible structure (Zheng et al., 2014). To further demonstrate the difference in mechanical behavior between the two types of structures, the characteristic stress-strain curves are shown in Figure 21.
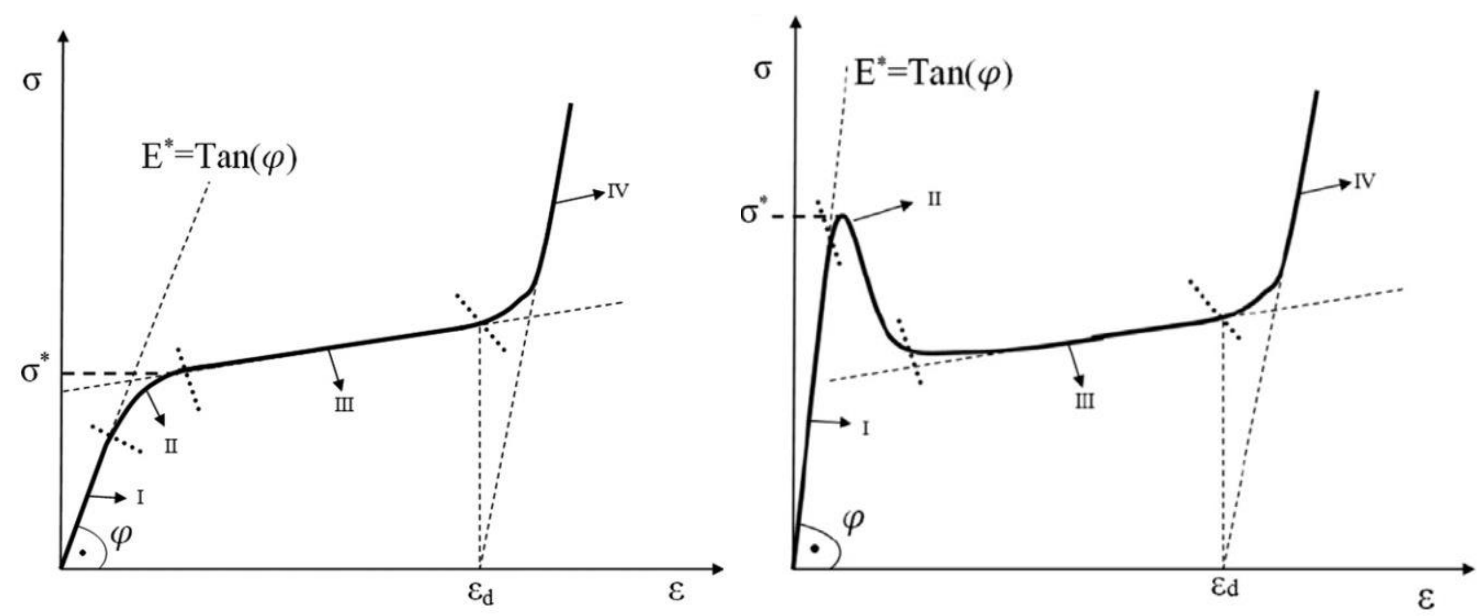

Figure 21: Characteristic stress-strain curves of (Left) bending-dominated and (Right) stretch dominated lattice structures (Recep Gümrük et al., 2013).

Each stress-strain curve can be broken up into four distinct zones: region I is the elastic response of the material, II is the elastic-plastic collapse, III is the plastic collapse region, and IV is the area where densification occurs. To study the mechanical properties of the structures, the size, thickness, and angle of the members in the unit cell are changed (Brøtan et al., 2016; R. Gümrük \& Mines, 2013; Recep Gümrük et al., 2013; Zheng et al., 2014). In general, compression tests were used to measure the mechanical response, but the main similarity between all of the studies is that they did not constrain their external edges. The lattice structures were built with a top and bottom layer, but never with external layers. The boundary condition at these layers influence the force path and deformation, as well as the local stress concentrations (Brøtan et al., 2016). 


\section{Initial Study}

To provide a general qualification of the electropolishing and laser polishing process, an initial study was performed. A test coupon was designed with the intention of being a design that could be used to evaluate many types of post-processing techniques, not just the ones investigated in this study. Due to budget constraints, only electrochemical polishing was evaluated using this coupon, while laser polishing was evaluated using a simple rectangular prism. The change in surface roughness and dimensions were used as the key performance indices in evaluating the processes. Additionally, the microstructure of the as-printed sample and the laser polished were investigated to observe the difference in grain size due to the different thermal histories.

\subsection{Electropolishing}

While electropolishing is generally a line-of-sight process, it can polish interior surfaces via electrolyte flow. Therefore, a sample with a mix of interior and exterior surfaces was designed.

\subsubsection{Sample Design}

Initially, the design considerations involved producing a test coupon that would be tested for both electropolishing and laser polishing. The main considerations in developing the coupon were producing a satisfactory number of different surfaces to test and having it be printable. The first iteration of the design can be seen in Figure 22. 


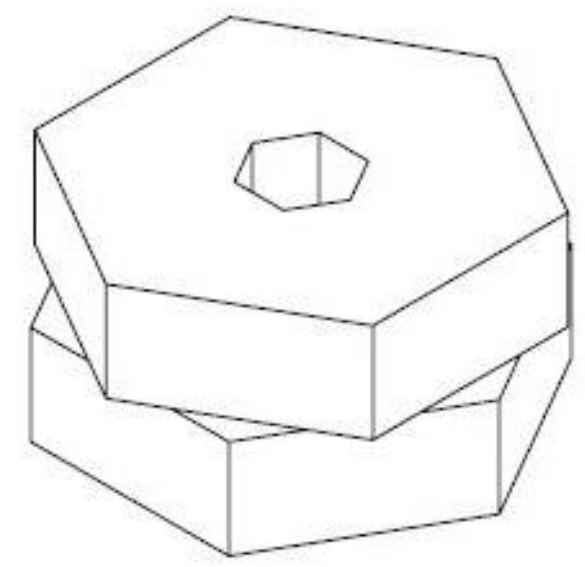

Figure 22: The first iteration of the double hexagon design, featuring a single, straight, hexagonal through hole.

The stacked hexagon design works well for an exterior polishing application; it has eight different surface angles with respect to the printing direction, giving a valuable amount of data points in between 0 and 90 degrees. However, the single through hole was not complex enough to give valuable information the ability of electropolishing to polish interior surfaces. Figure 23 depicts the next iteration of design.
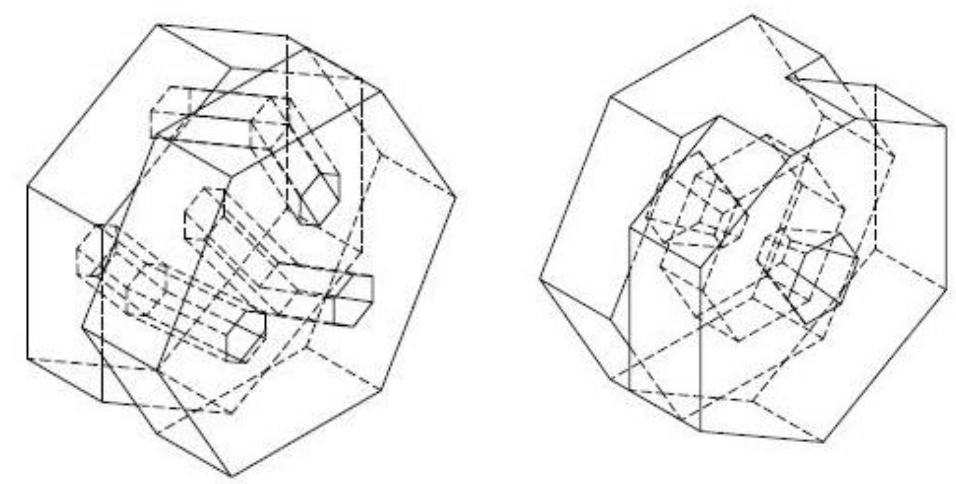

Figure 23: (Left) Stacked hexagon with a series of bent holes. (Right) Stacked hexagon with holes that taper into a cavity.

The stacked hexagon design was modified further with the addition of more complex holes, as well as removing a protruding corner to give the design a base. The complexity of the holes in both designs was satisfactory. However, the holes lacked variety, which 
would not have fully tested the capabilities of electrochemical polishing. Additionally, there still existed a significant amount of solid material that could be cut away to allow for more holes. Furthermore, using hexagonally shaped through holes meant that there were overhanging surfaces in the through holes that would generally require support structure to support. Figure 24 shows the final design.

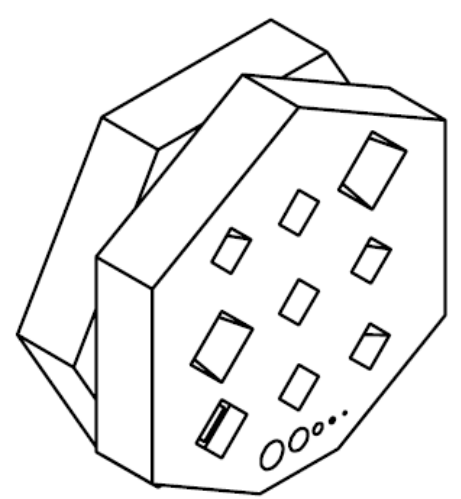

Figure 24: Stacked hexagon design with a variety of diamond through holes and a series of decreasing diameter circular through holes.

This design features a series of through holes. The section view can be seen in Figure 25.

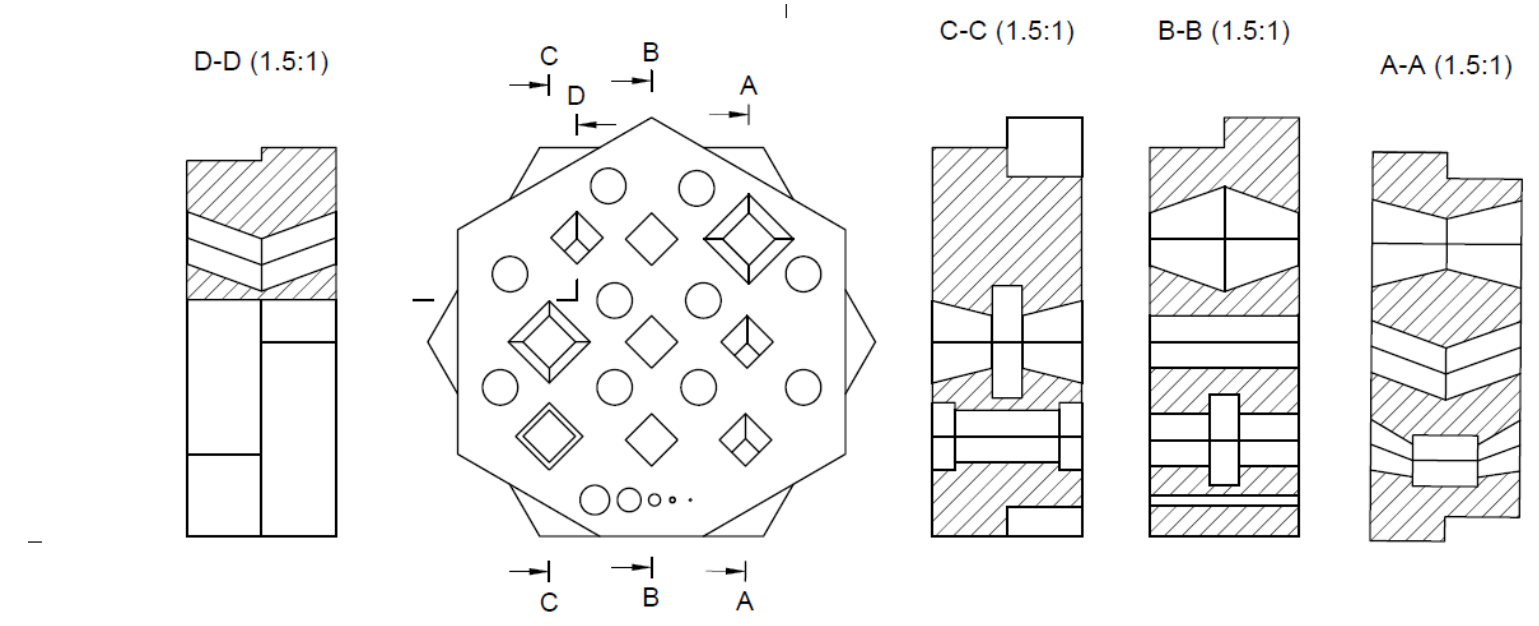

Figure 25: Section view of internal cavities of hexagon design. 
These holes were designed to provide a range of difficulties for the electropolishing fluid to flow through, as well as to represent a variety of use cases of holes that are unique to the additive manufacturing process. The large set of holes were designed to be a diamond cut with $45^{\circ}$ angles to prevent the need of support structure in the holes. The circular through holes were designed below an empirically determined diameter of requiring support structure. The labeling convention for the holes can be seen in Figure 26.
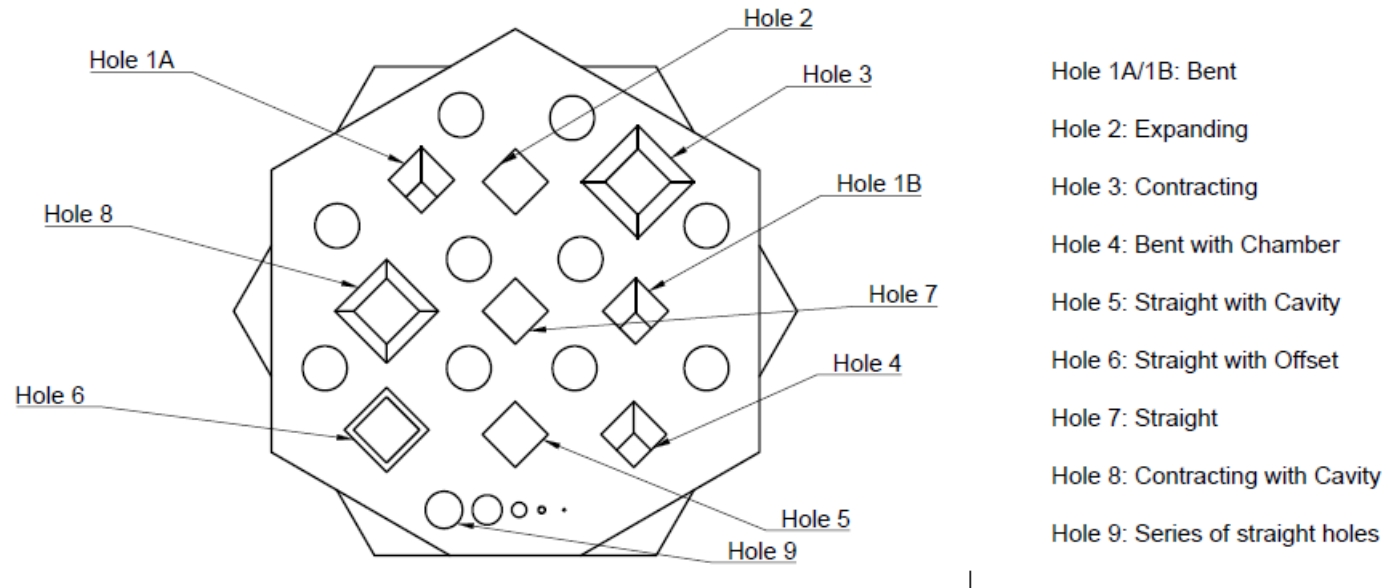

Figure 26: Labeling convention for the various holes for the electropolishing process evaluation coupon.

The dimension drawing for the model can be found in the Appendix A. The CAD file was imported into Magics and oriented as shown in Figure 27. 


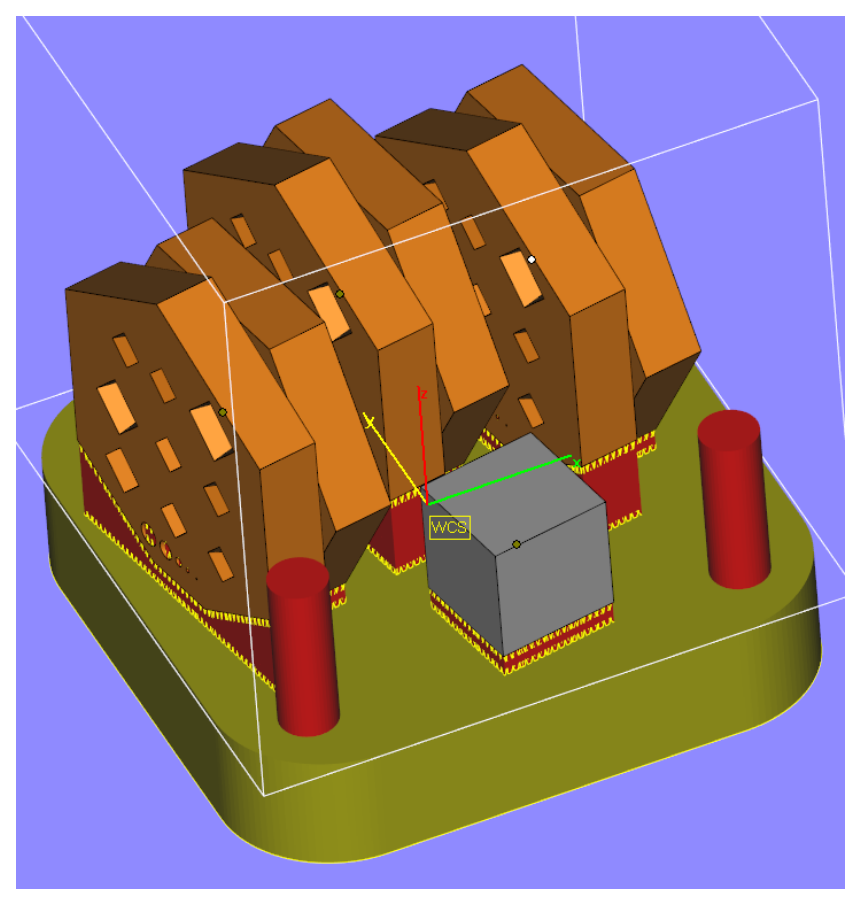

Figure 27: Magics output of three stacked hexagons and an early iteration of the laser polishing sample.

The auto-generated support structure from Magics was used. The entire build was supported by $5 \mathrm{~mm}$ of support structure to aid in the removal of the printed part, as well as guaranteeing the build was level. Support structure was used on the $30^{\circ}$ downward facing surface. The $60^{\circ}$ downward facing surface did not have any support structure supporting it. The printer used was the SLM 125 HL. The parameters used for the build are the default stripe parameters from SLM and are seen in Table III.

Table III: SLM 125 HL Key Printing Parameters.

\begin{tabular}{lllll}
$\begin{array}{l}\text { Hatch } \\
\text { Distance }\end{array}$ & $\begin{array}{l}\text { Layer } \\
\text { Height }\end{array}$ & Stripe Size & $\begin{array}{l}\text { Fill Contour } \\
\text { Laser } \\
\text { Power }\end{array}$ & $\begin{array}{l}\text { Fill Contour } \\
\text { Laser Speed }\end{array}$ \\
\hline $0.06 \mathrm{~mm}$ & $0.03 \mathrm{~mm}$ & $10 \mathrm{~mm}$ & $150 \mathrm{~W}$ & $450 \mathrm{~mm} / \mathrm{s}$
\end{tabular}

These parameters were determined experimentally for previous 316L builds on the same machine. The laser power and speed varied based on what type of feature it was 
scanning. The entire machine output can be seen in the Appendix B. The powder used for this build was spherical, with an average diameter of $50 \mu \mathrm{m}$, such as in Figure 28 .

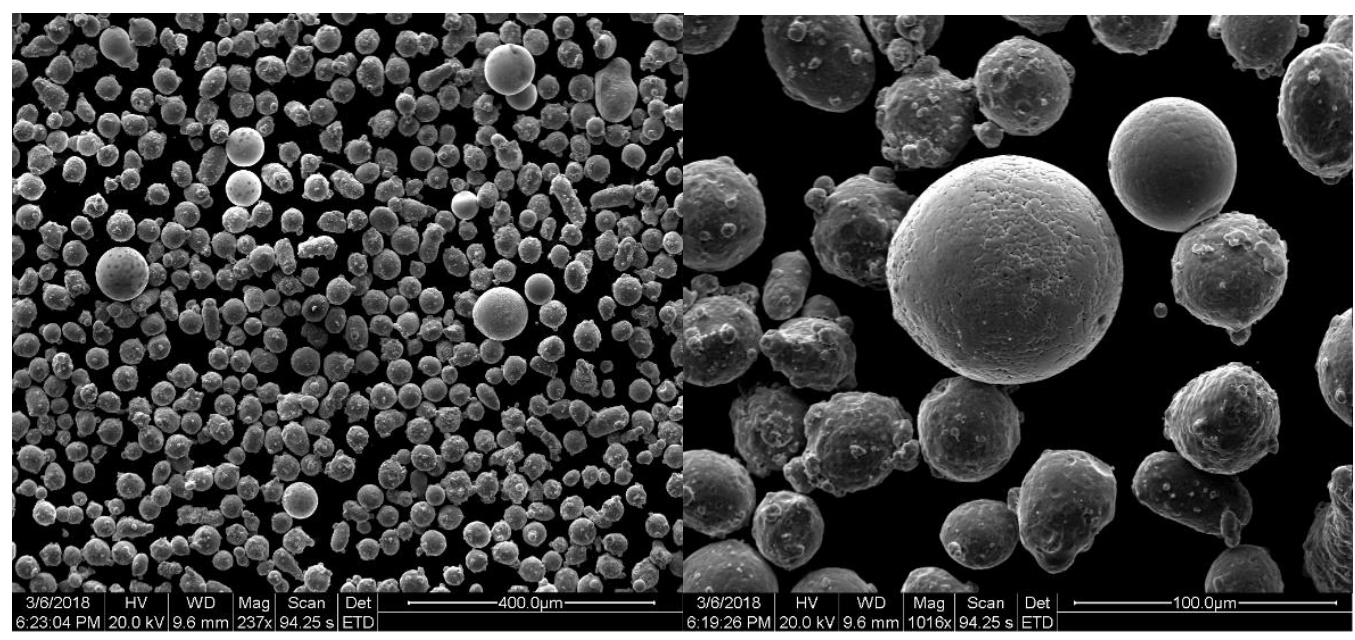

Figure 28: SEM image of a sample of the powder used for all of the builds.

Compositional analysis was not performed on this sample, but it is assumed to be nominal composition of $316 \mathrm{~L}$ powder. Unfortunately, the build failed on layer 352 due to a recoater position error. Figure 29 shows the failed sample in the build chamber, as well as outside of the build chamber. 

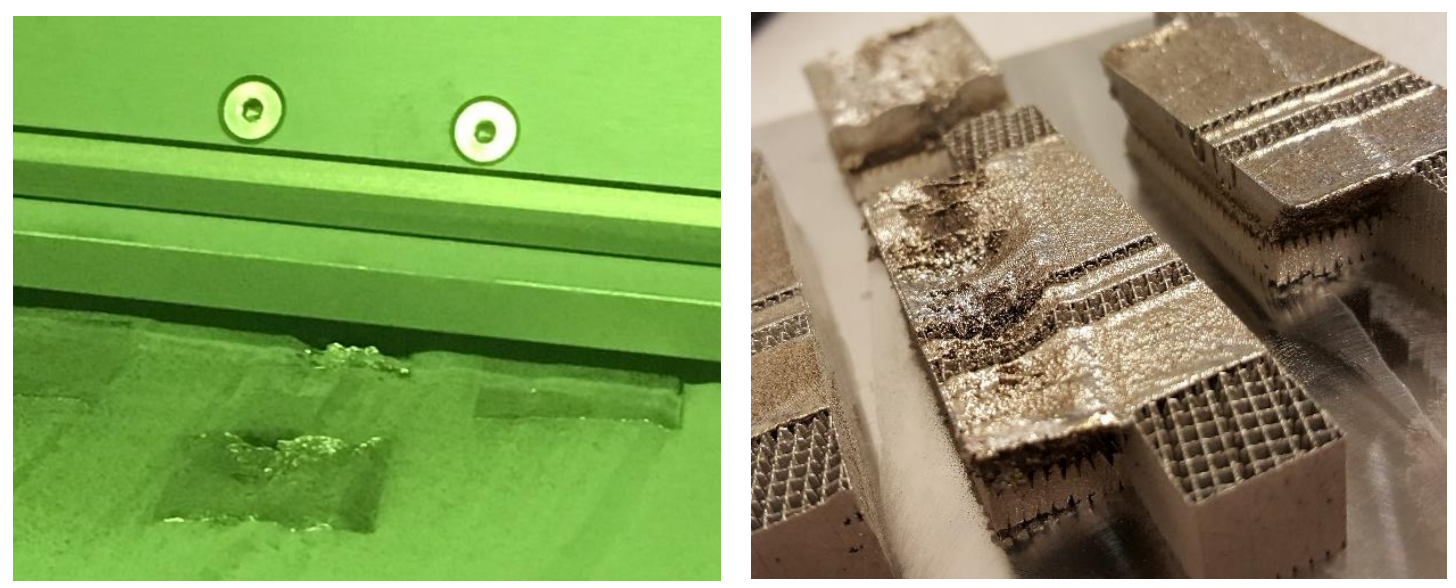

Figure 29: (Left) Failed build in build chamber. (Right) Failed build after removal from build chamber showing warpage, significant burn in, and missing material.

The signature burn marks and missing material indicate the failure of the recoater arm to move powder material into the correct position for the laser to melt. This caused the laser to burn the previously deposited material instead of melting new material. Upon careful examination of the failed build, it can clearly be seen that there was warpage of the material during the build that the recoater blade was snagging on, causing it to fail to deliver the powder. Warpage occurred at both the cube and the $60^{\circ}$ downward facing surfaces. The suspected root cause of the warpage was the inability of the build to properly dissipate the thermal energy imparted to it by the melting process. Several factors contributed to this: firstly, the packing factor of the build was too high, leading to a large buildup of heat on the build plate due to the amount of thermal energy needed to melt that much material. Secondly, the hexagons were largely composed of solid material, again contributing to a large amount of thermal energy to the build plate. Finally, the decision to not use support structure on the $60^{\circ}$ downward facing surface lead to an insufficient number of heat conduction paths for the printed material, again causing a large buildup of thermal energy. 
The part was re-designed and re-printed with these considerations in mind. Additionally, to improve the heat dissipation during the build, the time between layers was increased to 20 seconds. In order to remove material while maintaining a similar thickness throughout the sample, ten through holes were placed on the sample. These holes were printed with support structure in order to avoid removing too much material and inducing new residual stresses. The resulting, successful, print is shown in Figure 30.

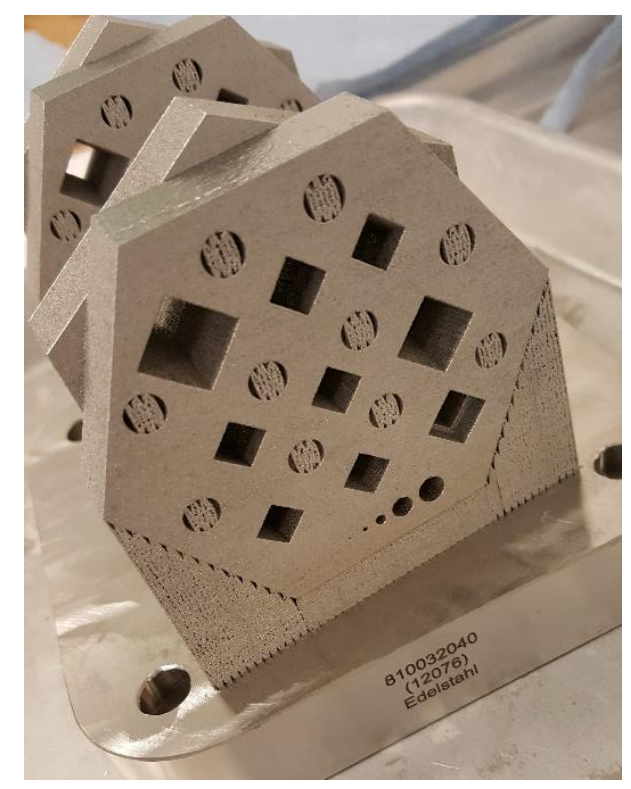

Figure 30: First successful build, still attached to build plate. Some cracking of the support structure can be seen.

While there are some burn marks present at the bottom of the build, this build was considered successful for testing purposes. The sample was removed from the build plate by passing a bandsaw through the support structure. Remaining support structure was removed with a hammer and chisel.

Additionally, a second build was printed to be used as a control sample for sectioning and metallography. However, this build failed approximately $2 / 3^{\text {rds }}$ of the way through due to an error with the filter. The failed build can be seen in Figure 31. 


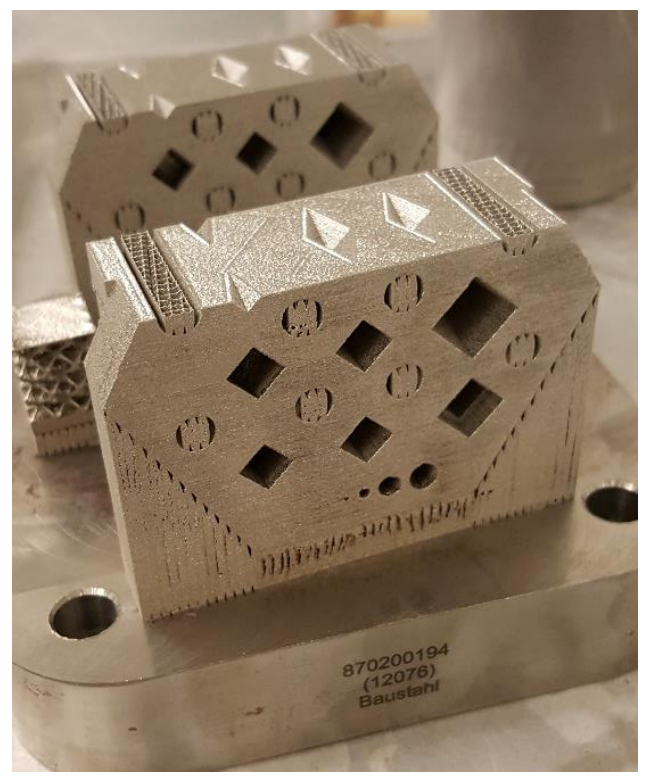

Figure 31: Unfinished build still attached to build plate after removal from build chamber.

Although the build was not allowed to continue, it is clear that what was printed was of high enough quality to be used for examination. Therefore, it was still used for its intended purpose.

\subsubsection{Electrochemical Polishing}

Once all necessary prior measurements were performed, a single hexagon was electropolished. Table III summarizes the parameters used for the electropolishing process. 
$\underline{\text { Table III: Electropolishing Process Parameters }}$

Bath Electrolyte Voltage Current Time in

Temperature

Density Bath

\begin{tabular}{|c|c|c|c|c}
\hline $130{ }^{\circ} \mathrm{F}$ & $\begin{array}{c}\text { Phosphoric } \\
+ \text { Sulfuric }\end{array}$ & Unknown & Unknown & 40 minutes \\
Acid & & & \\
\hline
\end{tabular}

Unfortunately, the electropolishing company was not willing to share any specific details about their process except for the general composition of the electrolyte and the bath temperature. However, they claimed that they had never had a problem with hydrogen embrittlement, so the voltage and current density can be assumed to be at or above the oxygen evolution potential of the anodic polarization curve. The orientation of the hexagon during the electropolishing process can be seen in Figure 32.

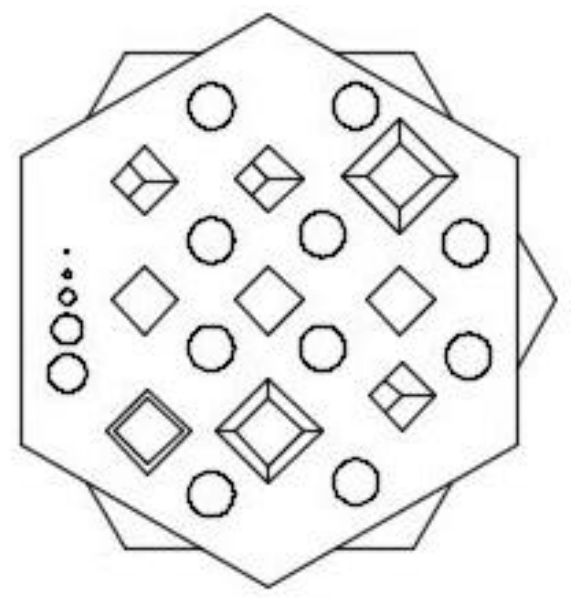

Figure 32: Orientation of hexagon during electropolishing. The electrolyte flowed out of the holes. 
The electrolyte flowed from back to front. The hexagon remained in this orientation for the entirety of the polishing process. Figure 33 shows the hexagon immediately following electropolishing.
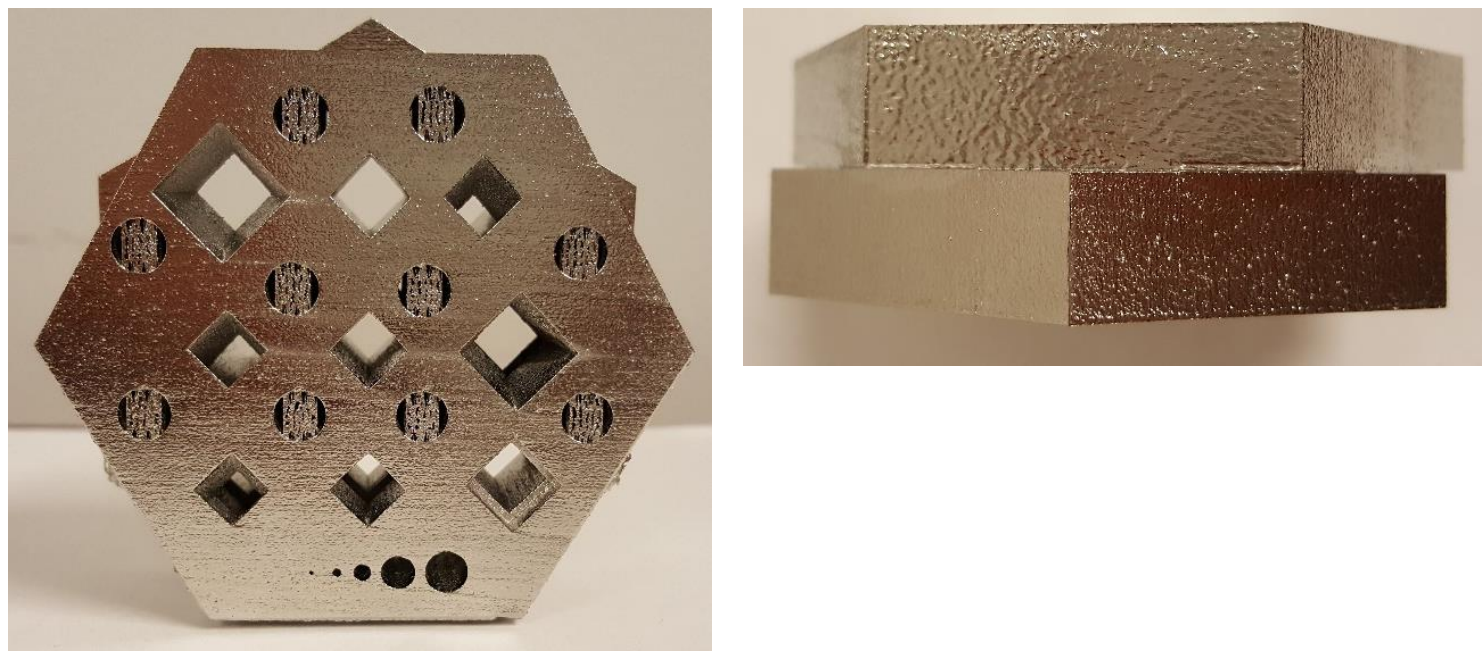

Figure 33: Front view and top view of hexagon immediately following electropolishing process.

When compared to the dull finish of the hexagon prior to polishing, the electropolished hexagon is significantly shinier, and felt much smoother to the touch. Additionally, some curvature of the previously sharp corners was visible with the naked eye.

\subsection{Laser Polishing Sample Design}

Due to the strict line-of-sight behavior of laser polishing, only exterior surfaces can be polished. Additionally, the laser polishing supplier wanted to work on a single surface with area 1 in. $^{2}$ to establish working parameters for future polishing. Therefore, a simple rectangular prism was designed. The design for the sample can be seen in Figure 34. 

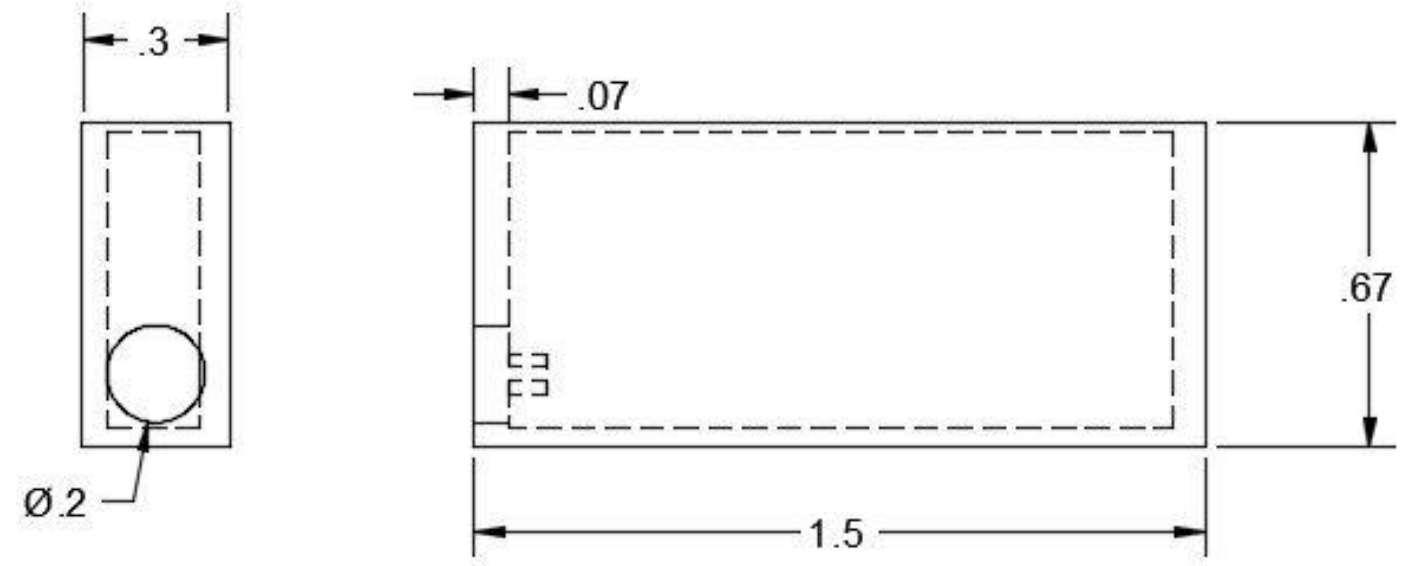

Figure 34: Laser polishing sample dimensions in inches.

The sample was designed to be a thin-walled structure to aid in its printability. Due to the hollow interior, a small diameter hole was added to allow un-melted powder to be removed. The hole was filled with support structure to reduce potential warpage during printing, as seen in Figure 35.

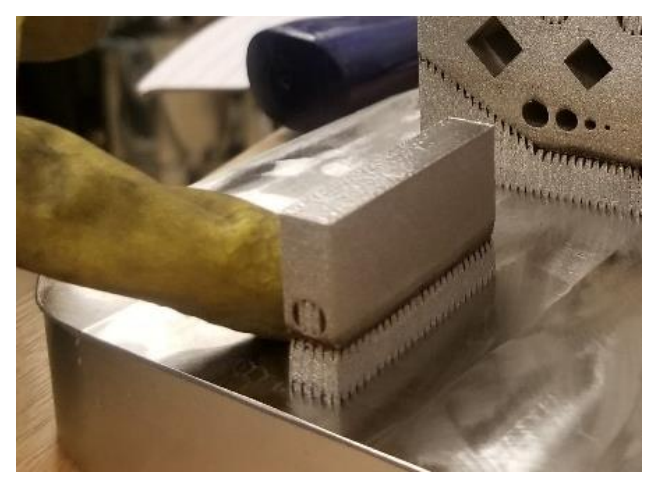

Figure 35: Laser polishing sample still attached to the build plate with a hexagon in the background.

A small amount of burning can be seen at the bottom of the build, but otherwise this build was successful. The support structure was removed from the hole, and the powder was removed. 
To establish working parameters for the process, two different energy densities were used while varying the total number of pulses the material received. The experimental parameters are shown in Table IV.

\section{Table IV: Laser Polishing Experimental Parameters}

\begin{tabular}{|c|c|c|}
\hline Beam Size & Energy Density & Total Number of Pulses \\
\hline $1.8 \times 1.8 \mathrm{~mm}^{2}$ & $1,4 \mathrm{~J} / \mathrm{cm}^{2}$ & $50,75,100,125,150,175$ \\
\hline
\end{tabular}

To optimize the parameter for surface roughness, one exposure per energy density and pulse combination was made in a series of small squares. The scanned areas were compared to the adjacent as-printed material. Once the optimal parameter was identified, it was used to scan stripes using different patterns. These patterns were scanned by holding the laser in place and moving the workpiece with motors. An excimer laser system was used for the testing.

\subsection{Methods}

To properly prepare the samples for both polishing and evaluation, a series of cleaning and cutting processes were performed. First, the powder and support structure were removed from the printed samples. Then, the surface roughness and dimensional measurements were performed. 


\subsubsection{Powder Removal}

Prior to removal from build chamber, as much powder as possible was removed from the samples by tilting and hitting with a hammer. To remove the remaining powder, the sample was cleaned in an ultrasonic bath of distilled water. Powder removal occurred via the impingement of bubbles.

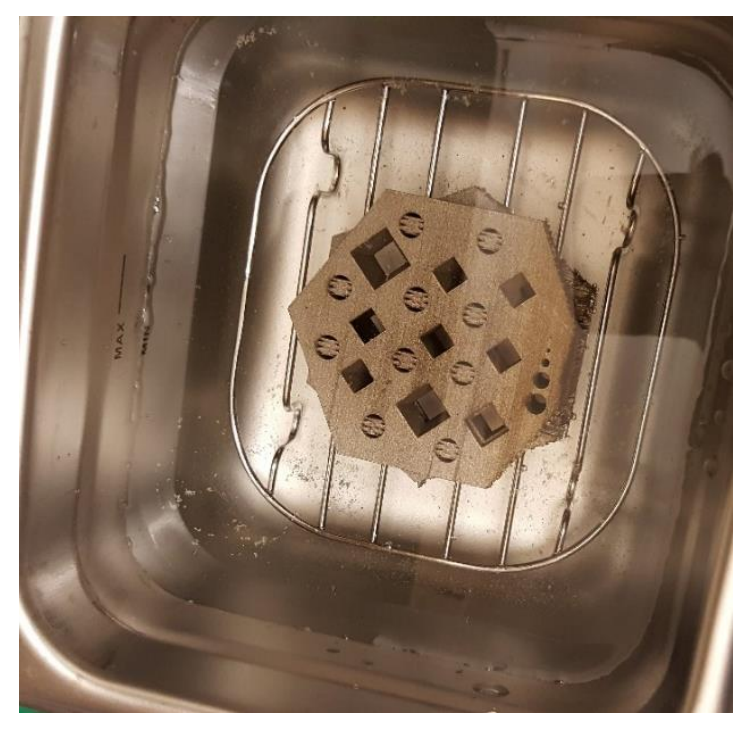

Figure 36: Immediately following support structure removal, the sample was ultrasonicated.

The sample was ultrasonicated for 10 minutes per face for a total of 20 minutes.

Immediately following removal from the bath, the sample was dried with a heat gun.

\subsubsection{Surface Roughness Testing}

All available surfaces were measured using a Mitutoyo SJ-201 Profilometer. Prior to measurements, the profilometer was calibrated to a test block with a known $R a$ of 2.94 $\mu \mathrm{m}$. Unfortunately, due to the presence of support structure stubs, the surface roughness 
of all surfaces with support structure attached to them were unable to be measured. The profilometer was calibrated prior to each use.

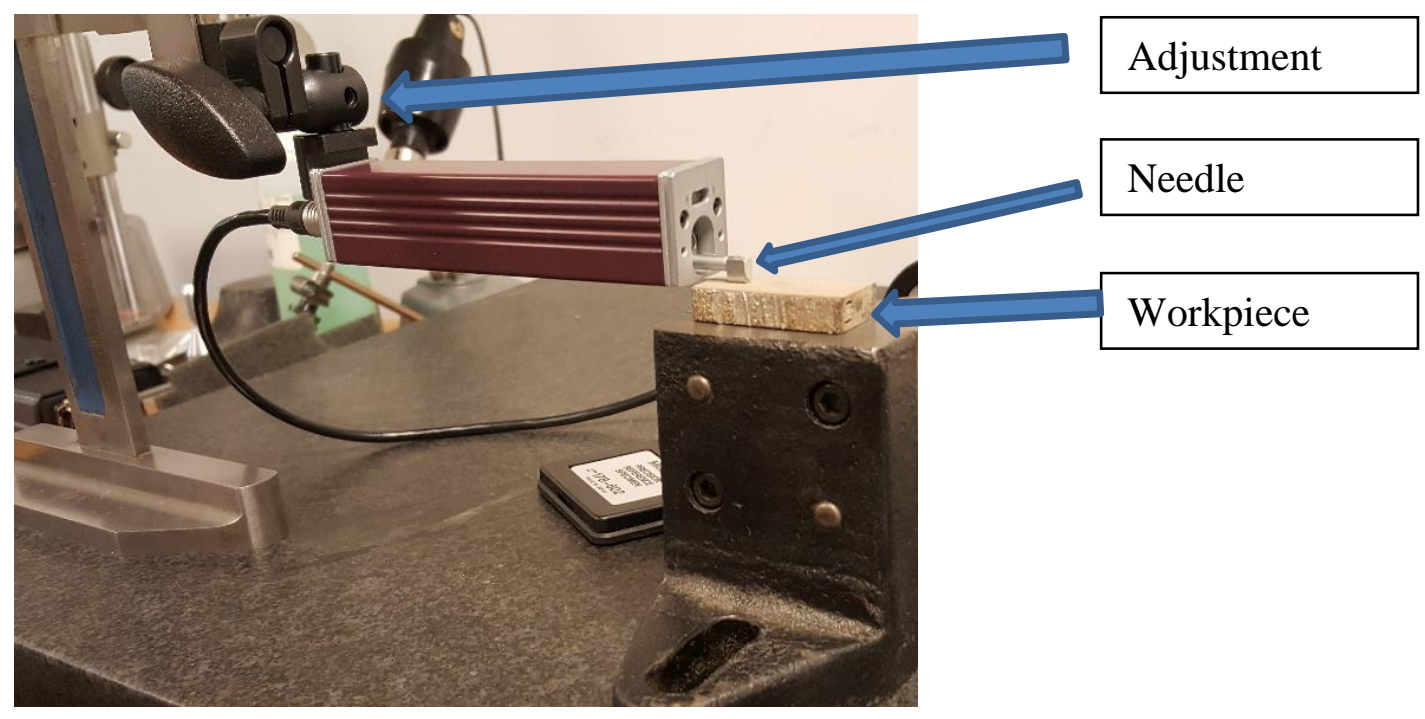

Figure 37: Outside surface profilometry setup.

A minimum of three measurements was taken for each face. $R a$ and $R z$ were recorded in $\mu \mathrm{m}$. When necessary, roughness measurements were taken both parallel and perpendicular to the build direction. In order to measure surface roughness of the interior surfaces, the profilometer was tilted to a $45^{\circ}$ angle.

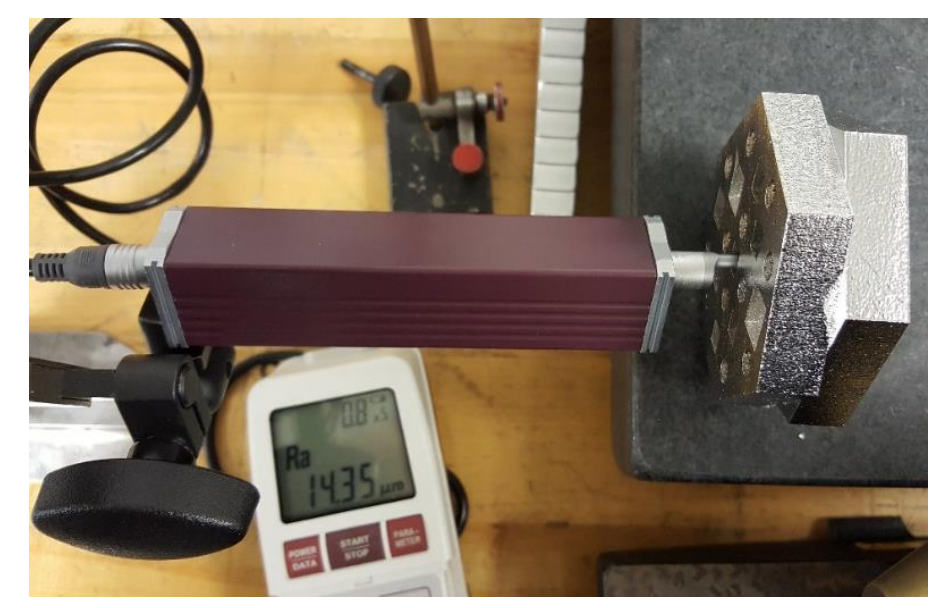

Figure 38: Profilometer setup for interior surface roughness measurements. 
However, the profilometer was only able to measure the interior surface roughness of the intact hexagon for three holes. Therefore, the part was sectioned to open the holes, as can be seen in Figure 39.

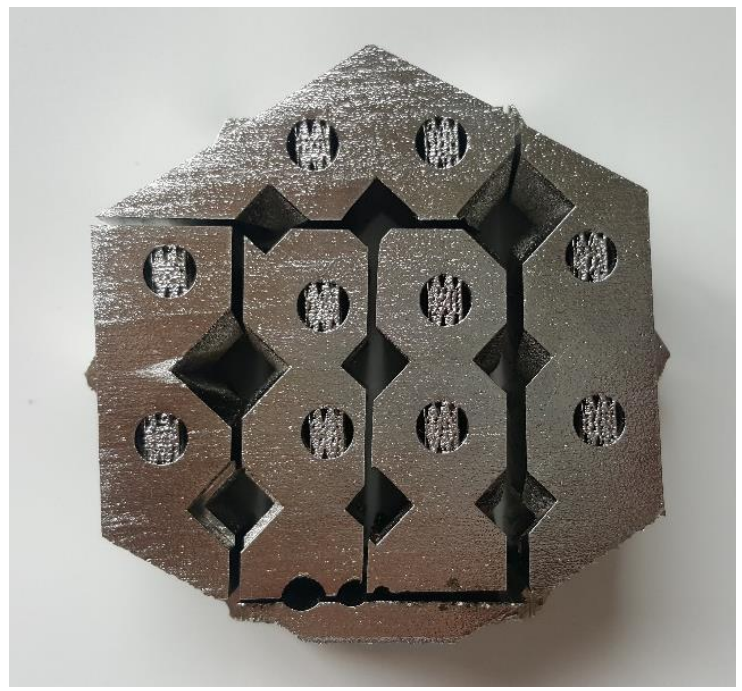

Figure 39:Hexagon immediately following sectioning.

A metal cut-off saw was used for sectioning. Due to the width of the saw, the three smallest diameter through holes were mostly destroyed, making measurements impossible. However, the two largest holes remained and were measured.

\subsubsection{CMM}

To measure the dimensions of the parts produced, a Micro-Vu Vertex 312c and the accompanying InSpec Metrology software was used. 


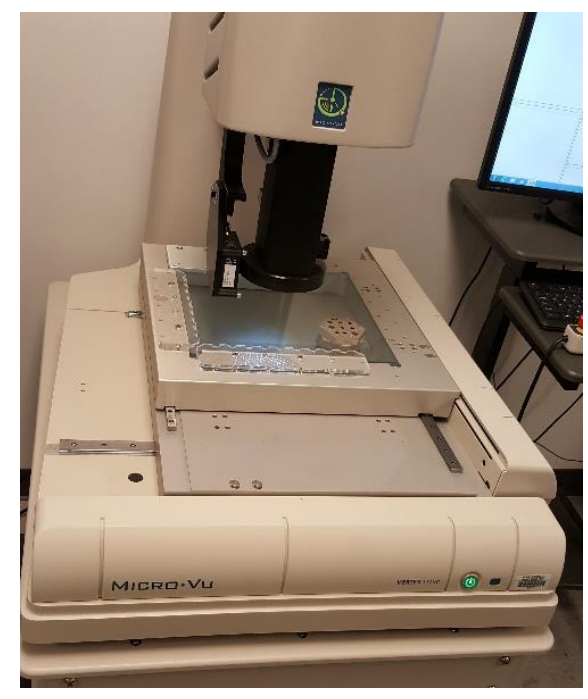

Figure 40: Hexagon loaded on machine, prior to imaging.

The light levels were adjusted on a per-sample basis to obtain the best contrast between solid material and empty space. Features were measured by having the computer recognize where the light levels differed from each other, indicating an edge or other type of feature.

\subsubsection{Scanning Electron Microscopy}

All scanning electron microscopy was performed using an SEM FEI Quanta 200. The images were gathered using the Everhart-Thornley detector. Accelerating voltage, spot size, and magnification were changed on a per image basis, and are included in the marker bar at the bottom of all SEM images.

\subsubsection{Metallography}

To prepare samples for metallography, they were sectioned and then mounted in bakelite. Samples were then ground and polished to a $0.5 \mu \mathrm{m}$ finish. Samples were then 
electrolytically etched at $3.1 \mathrm{~V}$ and $1.5 \mathrm{~A}$ for 40 seconds in a solution of $10 \mathrm{wt} \%$ oxalic acid. Microstructures were evaluated using the scanning electron microscope.

\subsection{Results}

The results for electropolishing and laser polishing were similar to what was expected from literature, but typically fell short of expectations. While there was certainly a large amount of material removal during electropolishing, the smallest surface roughness achieved was approximately $3 \mu \mathrm{m}$. Laser polishing was able to achieve surface roughness values of approximately $2.5 \mu \mathrm{m}$. For both processes, research reported the achievable surface roughness to be on the order of nanometers (Urlea \& Brailovski, 2017;

Willenborg, 2011).

\subsubsection{Electropolishing}

This section details the results of the electropolishing portion of the study. First, microscopy results are discussed, which includes optical and scanning electron microscope images. Following that, dimensional analysis results are discussed. Finally, surface roughness results are shown. 


\subsubsection{Microscopy}

Samples were imaged before and after electropolishing. Figure 41 shows a magnified image of an interior, upward facing surface of the hexagon sample prior to polishing.

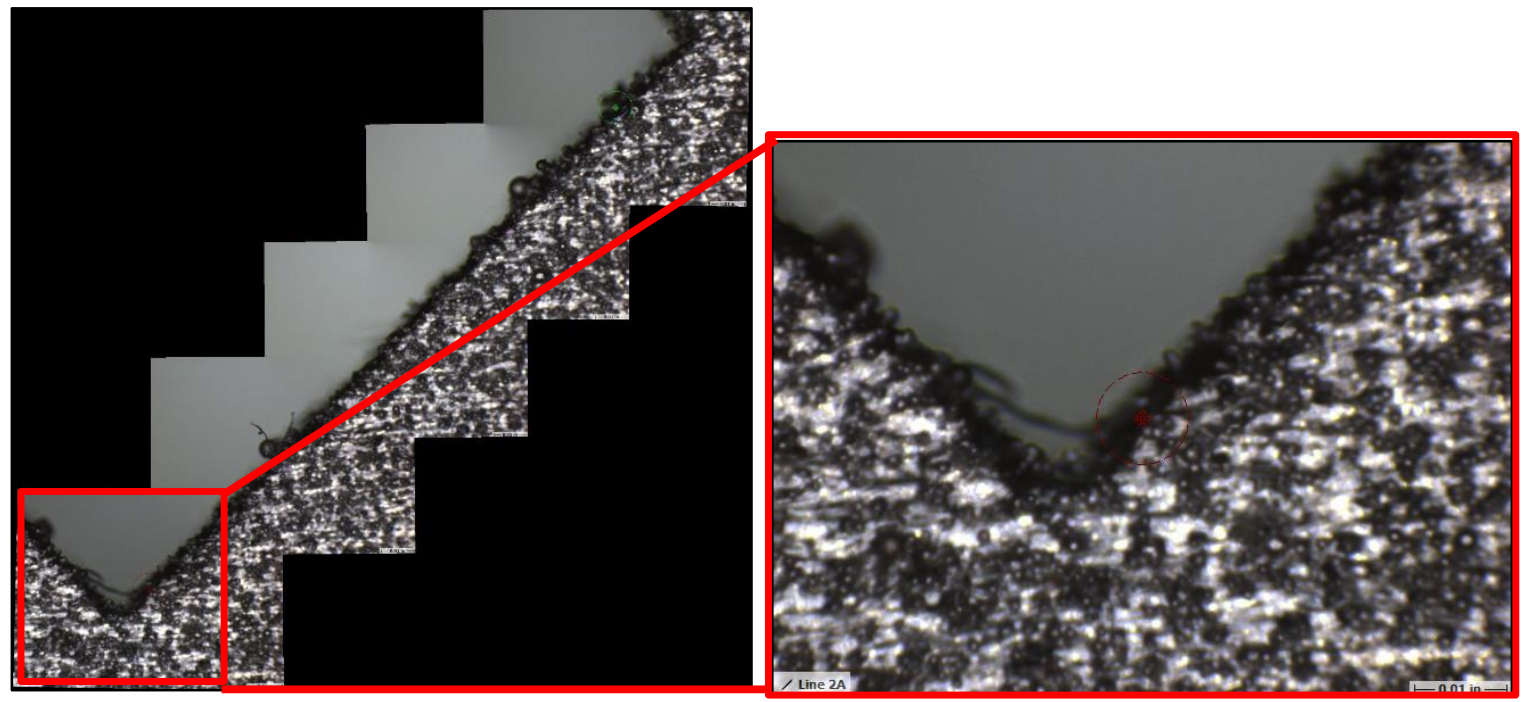

Figure 41: Multiple CMM images stitched together, depicting a single image's contribution to the whole.

The parasitic powder material can clearly be seen on these surfaces. Due to these surfaces being upward facing, most of the skin growth occurs from the excess thermal energy causing a solid-state sintering of neighboring particles in the powder bed. The same corner, post electropolishing, can be seen in Figure 42. 


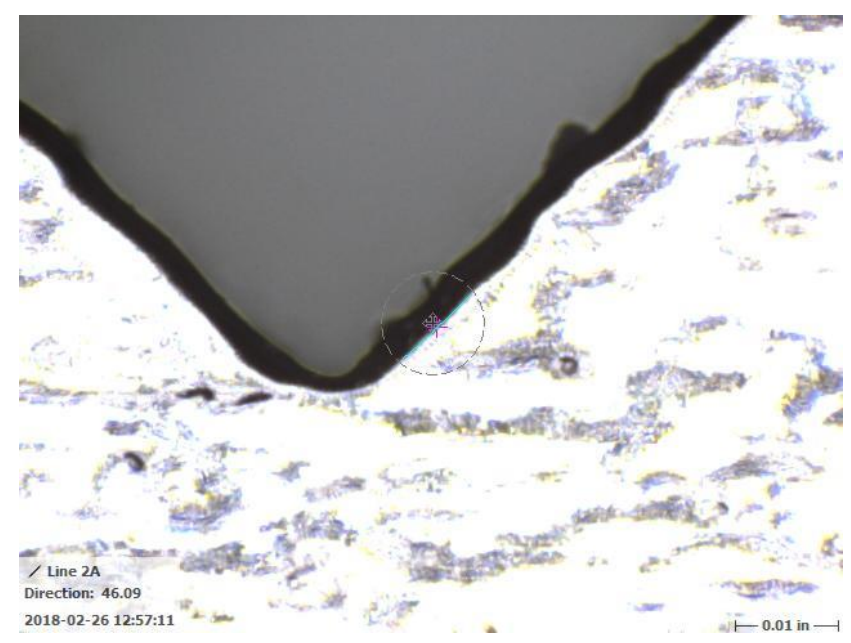

Figure 42: CMM image of a corner of electropolished hexagon showing an apparent increase in angle.

As can be seen in the image, a large amount of material removal occurred during electropolishing. The edges are much better defined, and the surface has become bright and reflective. To see the loss of material in the polishing process, the images were overlaid and subtracted from each other. Figure 43 shows the resulting image.

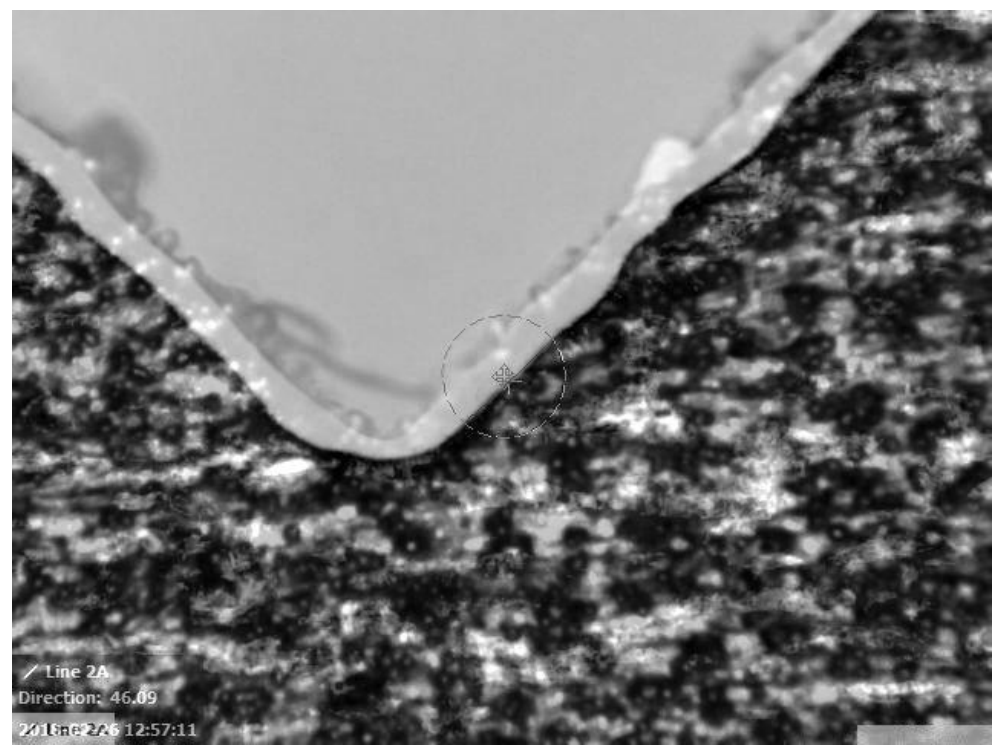

Figure 43: The difference between the two polished surfaces. 
This image shows that while the electropolishing process removed a significant amount of parasitic powder material, the dimensional stability stayed rather intact. While the inside corners experienced a small amount of rounding, some of the outside corners experienced a much larger amount, as seen in Figure 44.

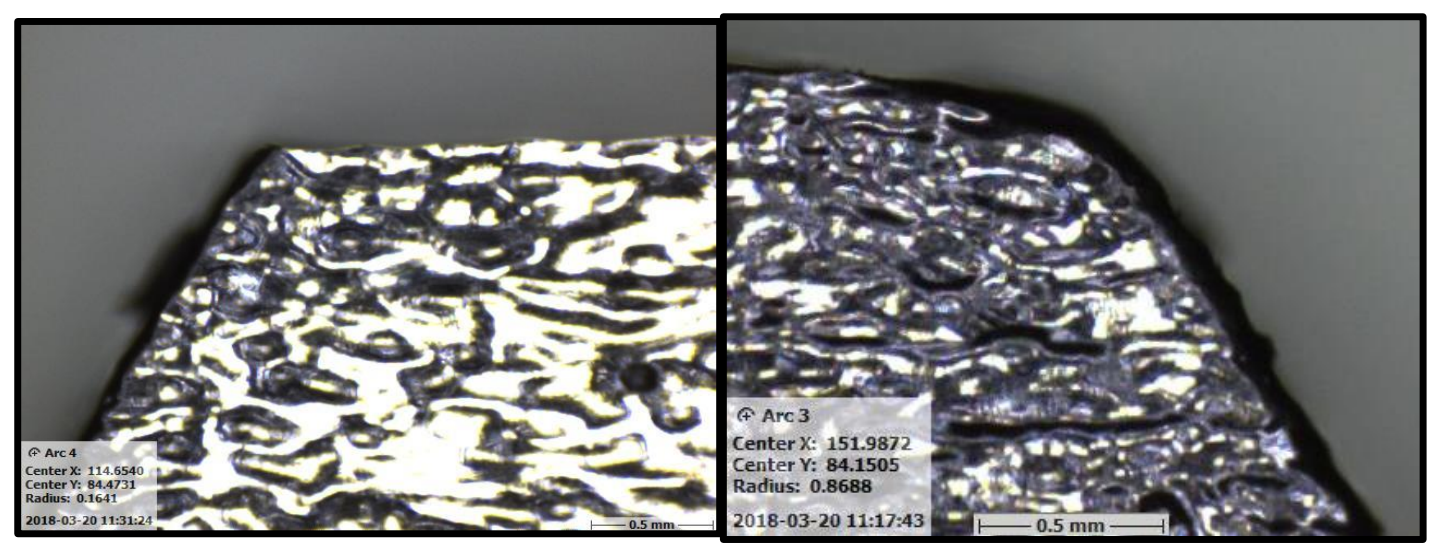

Figure 44: CMM image of the top left and top right corners of the electropolished hexagon.

The measured radius for the upper-right corner was four times as large the upper-left corner. Some of this variation may be due to the sample not being perfectly flat - this caused the profile and axial lighting of the CMM to make the edge look further in that it truly was.

SEM analysis showed that while the outer surface had been mostly cleaned of powder particles immediately following electropolishing, the interior surfaces still had quite a bit of powder remaining. Figure 45 and Figure 46 shows these resulting, powder covered surfaces. 


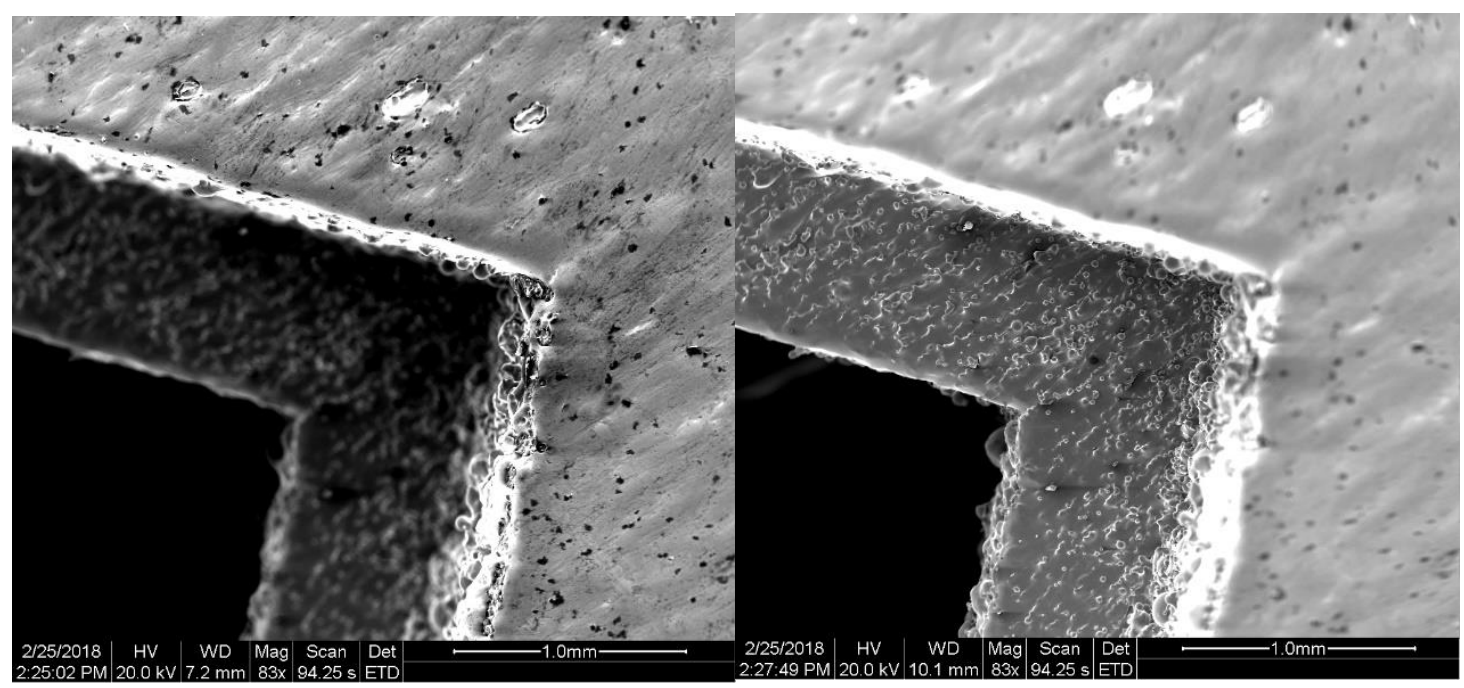

Figure 45: 83x magnification SEM image of an electropolished corner, showing a clean exterior surface with a powder covered interior surface.

While the amount of parasitic powder particles appears to have decreased slightly, it can still be seen that there is a large difference in the resulting surfaces, even though they share the same orientation with respect to the build direction.

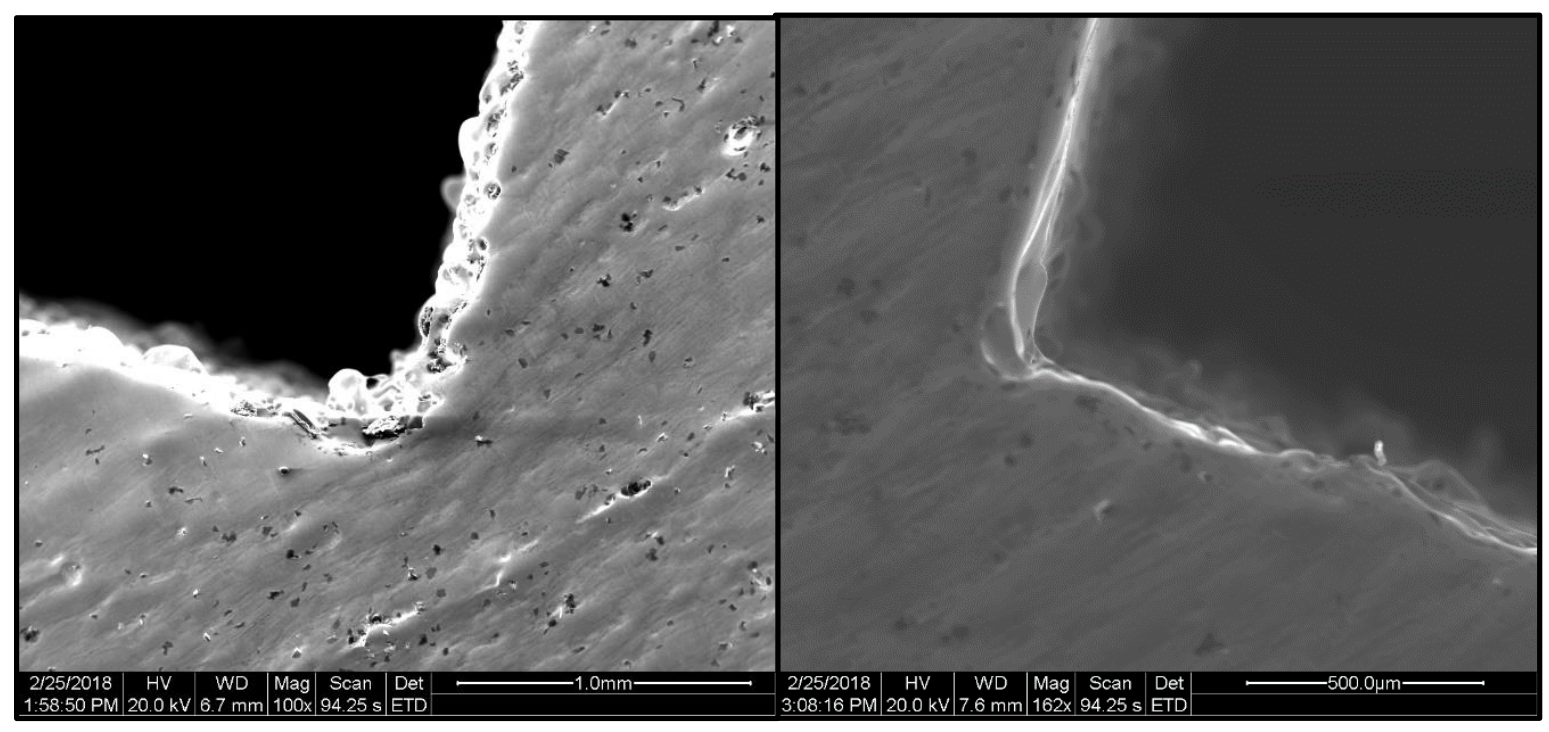

Figure 46: (Left) 100x SEM image with apparent powder particles remaining. (Right) 162x SEM image showing tear out at corner. 
Additionally, the electropolishing process had difficulty removing larger powder deposits. Figure 47 shows the interior of the $6 \mathrm{~mm}$ hole immediately following electropolishing.

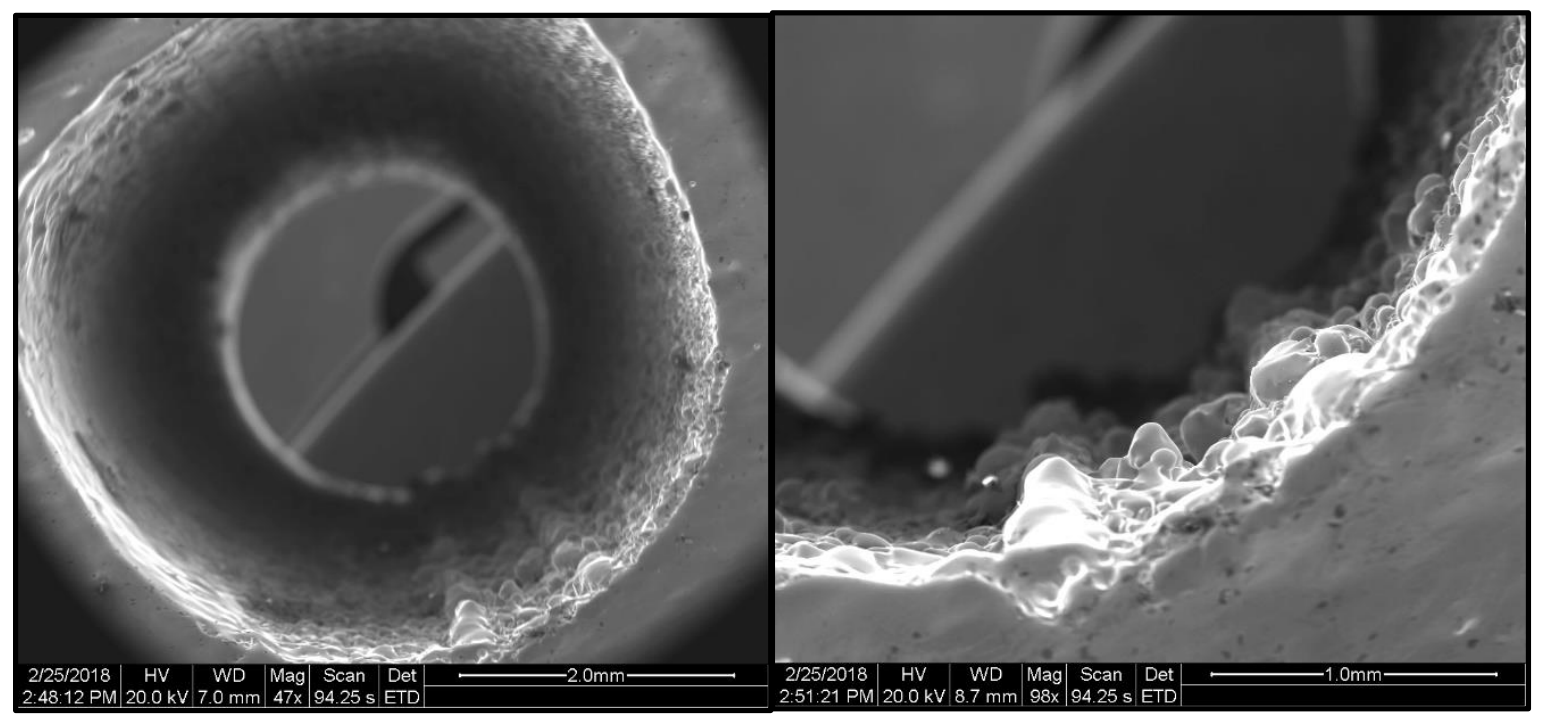

Figure 47: SEM image of $6 \mathrm{~mm}$ hole immediately following electropolishing; large parasitic powder deposits are clearly visible.

A small amount of surface roughness can be seen across the surface of the hole, but the downward facing surface still has a large amount of particle deposits. While the surface roughness appears to be minimal at the locations that are not the downward facing surface, Figure 48 shows that the electropolishing process was only able to remove material close to the entrance of the holes, but had difficulty penetrating all the way through the hole. 


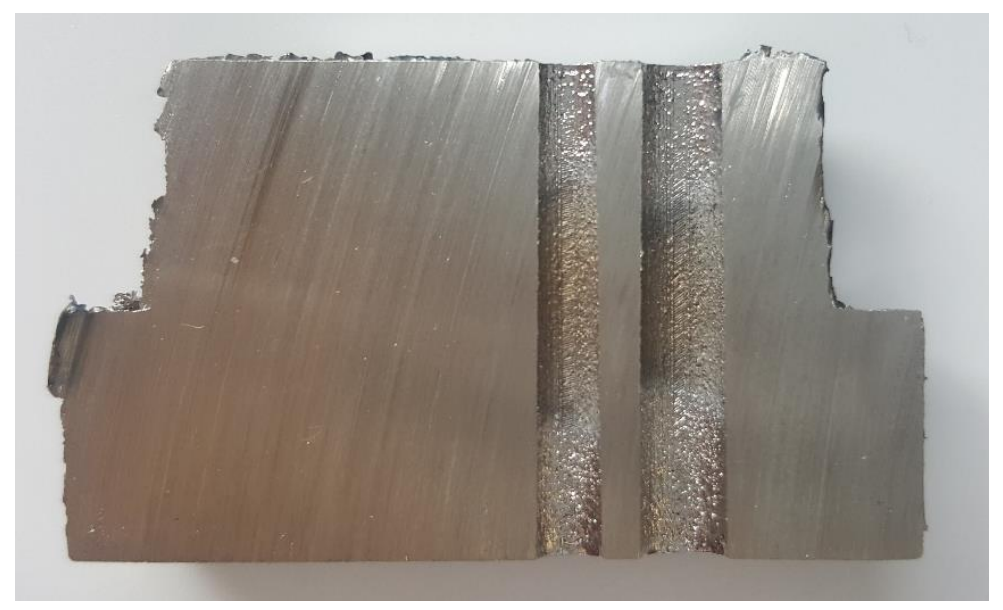

Figure 48: Sectioned interior of two largest diameter through holes of electropolished hexagon, showing lack of penetration of polishing. Electrolyte entered the holes at the bottom of this picture and flowed out the top.

While it is clear that the larger diameter hole had deeper polishing penetration, the

difference between the two holes is not significant. Additionally, the length of

electropolishing was slightly unequal; the electrolyte was able to provide slightly more

polishing at its entrance to the hole, while the opposite side relied solely on the potential

applied to it by the cathode. Scanning electron microscope images of the two holes can be seen in Figure 49.
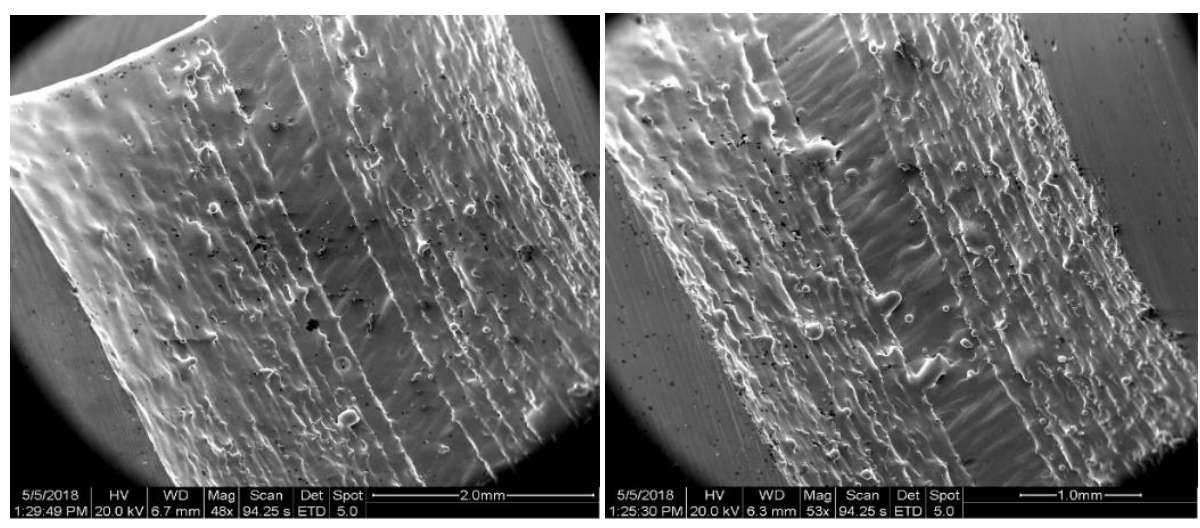

Figure 49: SEM images of upward facing surfaces of (Left) $5 \mathrm{~mm}$ hole at 48x and (Right) $4 \mathrm{~mm}$ hole at $53 \mathrm{x}$. 
The staircase effect is quite clear in these images; it is not as pronounced in the larger diameter hole as the smaller one due to the smaller increase in height required per layer. The $0^{\circ}$ surface for both samples is much smoother than all of the surrounding surfaces. Additionally, it can be seen by the smoothness of the remaining particles that some material has been removed, but because they are still there, there was not enough polishing action to remove everything.

The microstructure of the as-printed sample was evaluated in the perpendicular and parallel directions with relation to the build direction. Figure 50 shows the microstructure in the parallel direction.
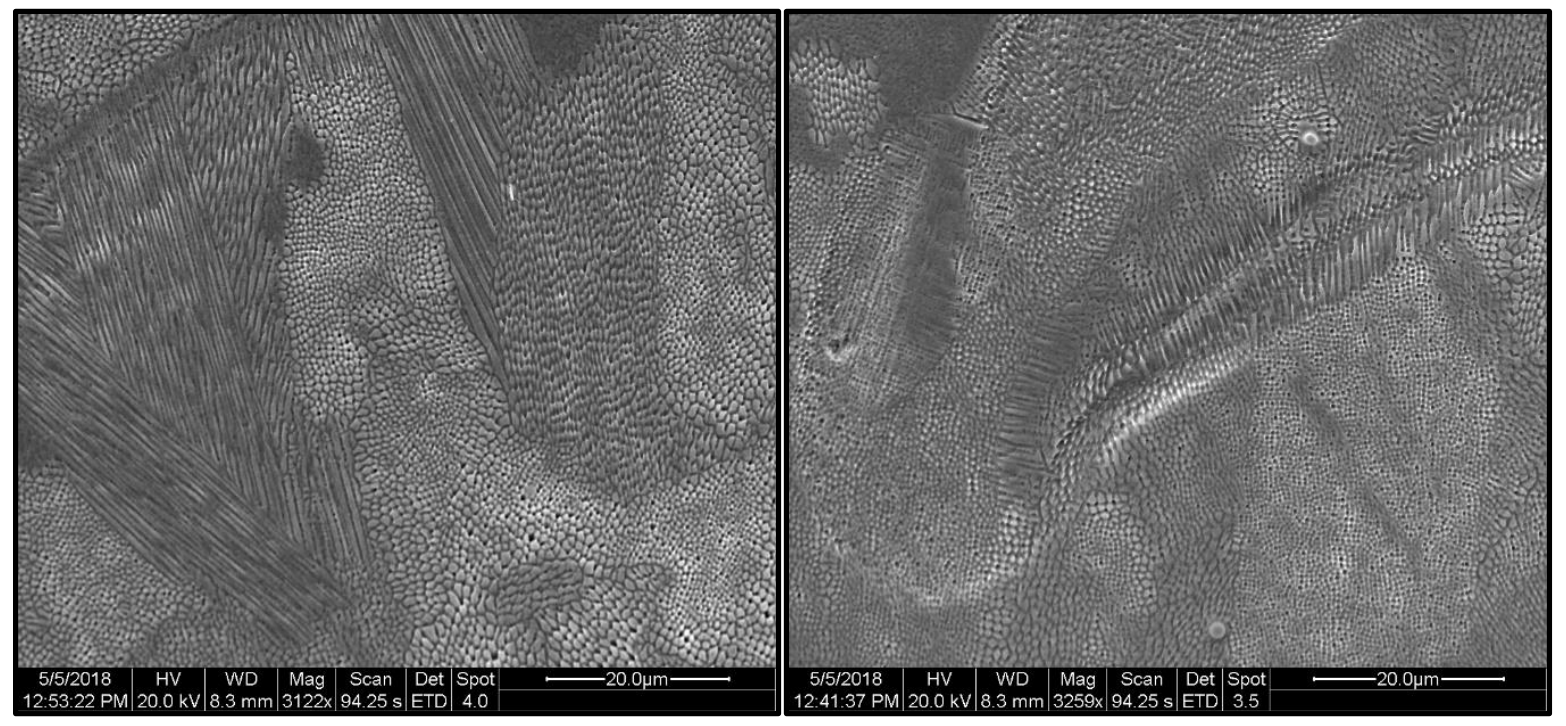

Figure 50: SEM images of etched microstructure, parallel to the build direction. (Left) 3122x magnification, showing both transverse and in-plane columnar growth. (Right) 3250x of an undercooling spine.

The microstructure displays the typical cellular structure observed in selectively laser melted samples. This structure is associated with the high levels of cooling and nonequilibrium solidification that occurs during the selective laser melting process. The microstructure on the right shows columnar growth due to the steep thermal gradient 
experienced in the process. This reinforces the influence of the thermal gradient on the preferred orientation of the grains. Following the etching of the parallel microstructure, the perpendicular microstructure was etched as well, shown in Figure 51.

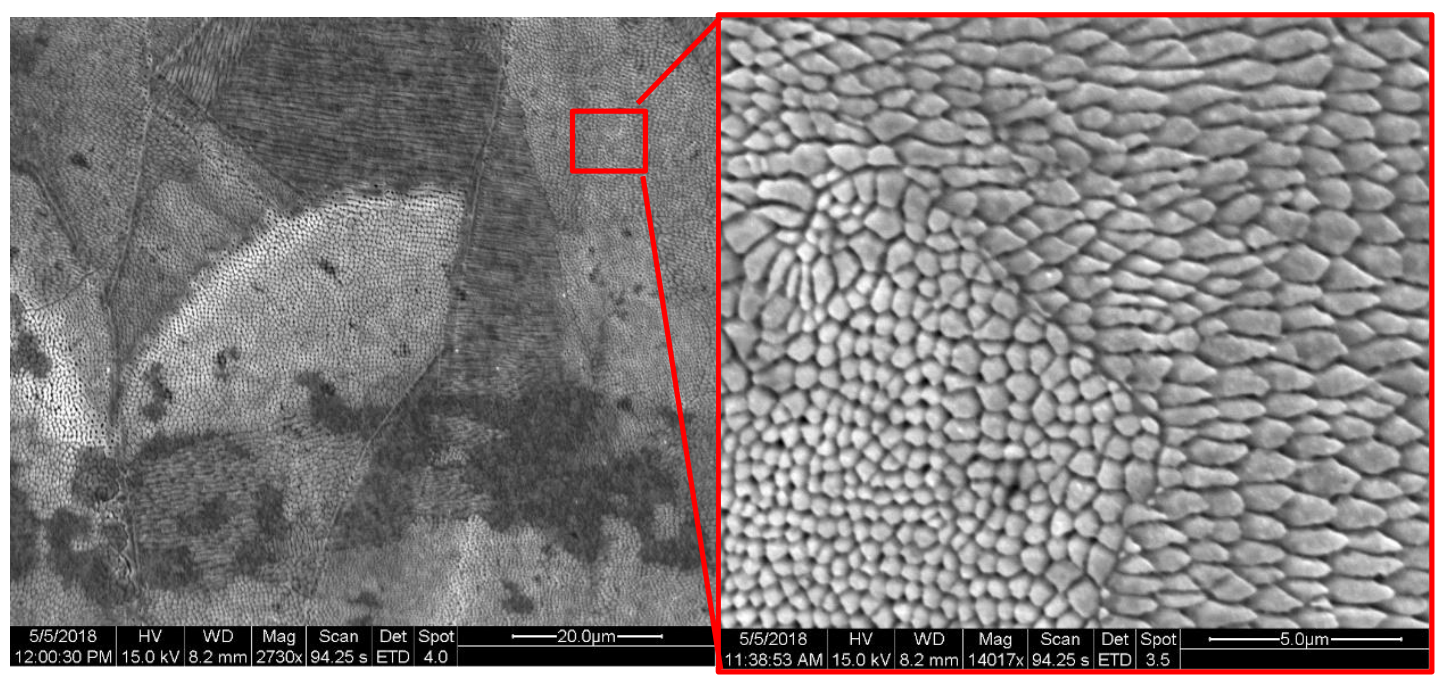

Figure 51: SEM images of etched microstructure, perpendicular to the build direction. High magnification image shows sub $5 \mu \mathrm{m}$ sub-grain size.

As seen in the high magnification image, the cells of the grains vary in size but do not seem to exceed $2 \mu \mathrm{m}$ in size. They appear to be equiaxed and oriented in the direction of the thermal gradient.

\subsubsection{Dimensional Analysis}

To measure the loss in material, measurements were taken before and after the polishing process. The main consideration was the change in roundness of corners from the asprinted state to the electropolished state, but it is still important to consider the asdesigned hexagon as well. When labeling the different corners, the convention in Figure 52 was followed. 


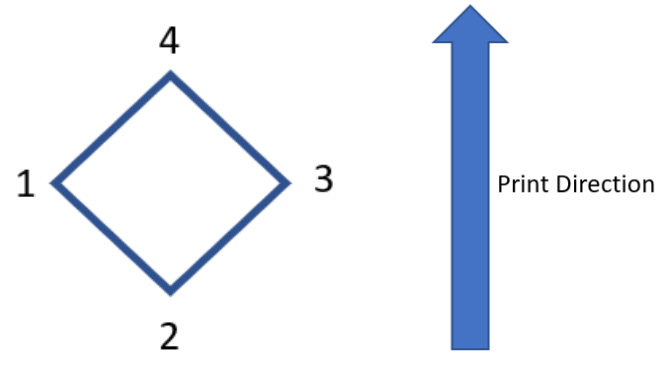

Figure 52: Labeling convention for corners of the through holes.

The corners were labeled starting with one on the left, increasing by one while moving counter-clockwise. When considering the through-holes, the ideal measurement for the radius of each corner should be zero, with an angle of $90^{\circ}$. Figure 53 shows the interval plots of both measurements for the average of both the pre- and post-processed states.
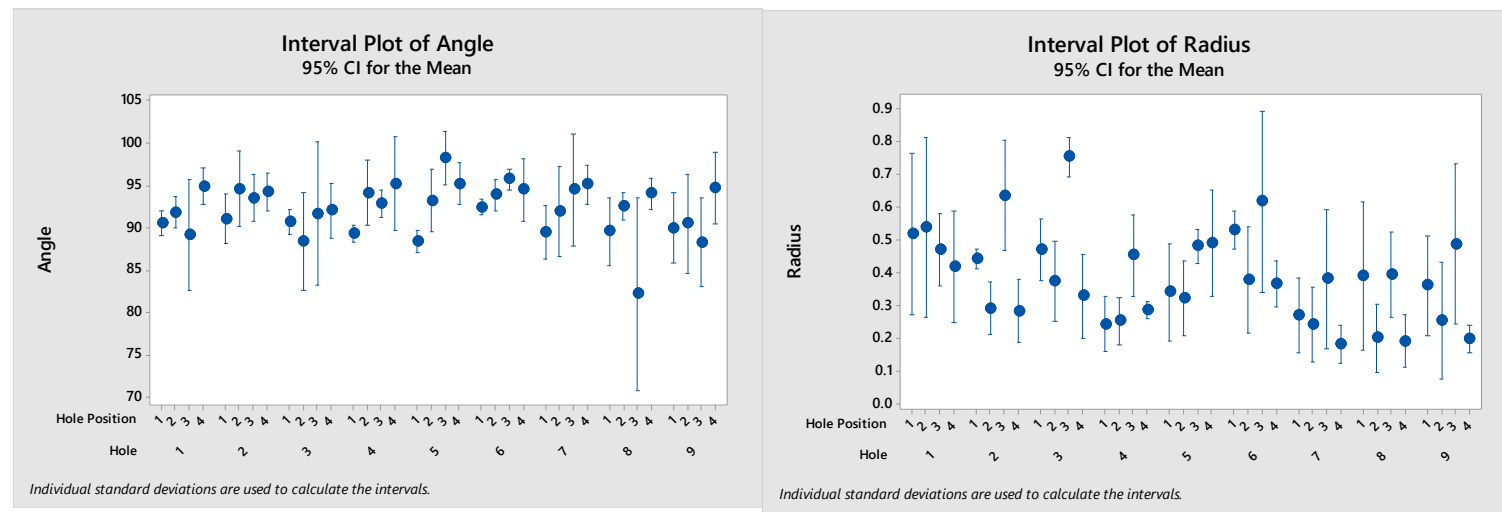

Figure 53: Interval plots by hole position and hole number for the measured angle (deg) and radii $(\mathrm{mm})$ of each corner.

It can be seen that the overall average of the angle measurements is, in general, slightly above $90^{\circ}$, while the overall average of the radius is clearly much greater than zero. The boxplots summarizing the data between the electropolished sample and the as-printed sample can be seen in Figure 54. 


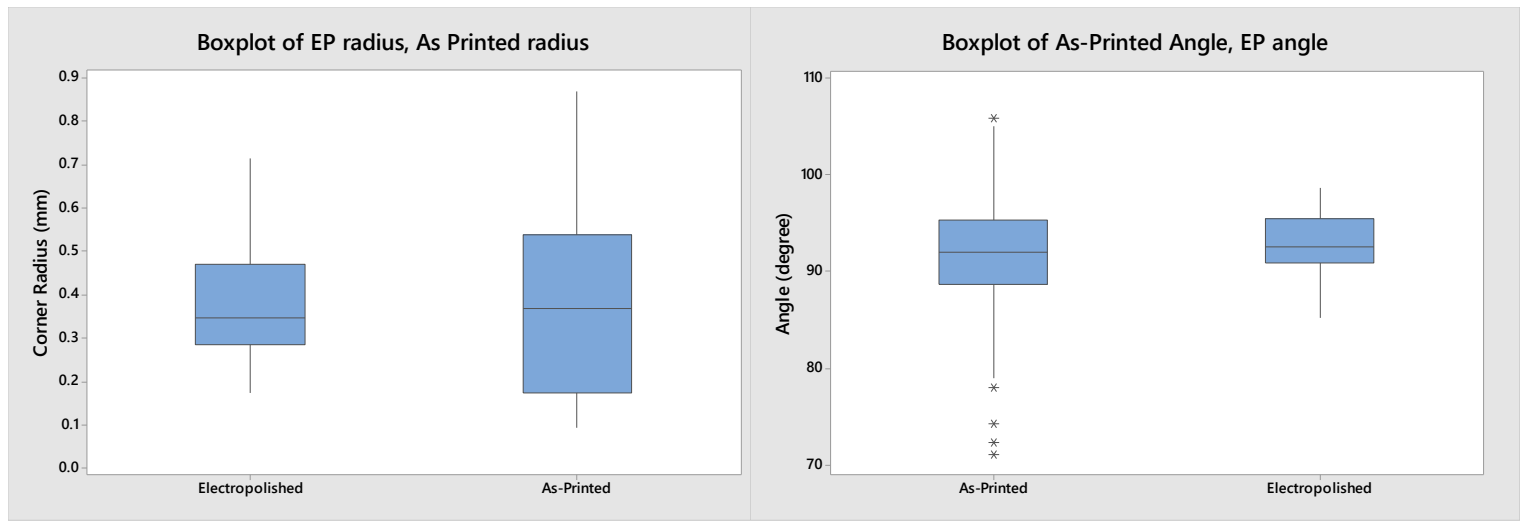

Figure 54: Boxplots of electropolished versus as-printed measured radius and corner angle.

While it can be seen that there is no significant difference between the electropolished sample and the as-printed sample, the as-printed sample shows much higher variance than the electropolished sample. This is primarily due to the parasitic powder particles interfering with measurements. An analysis of variance (ANOVA) model was produced for both radius and angle to determine if corner or hole had significant effects on the measured radius and angle. The ANOVA model for the electropolished radius measurements can be seen in Figure 55.

\begin{tabular}{|c|c|c|c|c|c|}
\hline Source & DF & Adj SS & Adj MS & F-Value & P-Value \\
\hline hole & 8 & 0.19416 & 0.024270 & 115.35 & 0.000 \\
\hline corner & 3 & 1.45327 & 0.484422 & 2302.25 & 0.000 \\
\hline hole*corner & 24 & 0.28778 & 0.011991 & 56.99 & 0.000 \\
\hline Error & 72 & 0.01515 & 0.000210 & & \\
\hline Total & 107 & 1.95036 & & & \\
\hline
\end{tabular}

Figure 55: ANOVA output for electropolished radius measurements, showing strong significance for the interaction between hole and corner.

Although the interaction of hole and corner is significant, the large F-value of the corner term indicates that it may be dominating the interaction. However, the measurements 
were highly repeatable, as indicated by the low value of the error term. The four-in-one plot, seen in Figure 56, validates the assumptions of the ANOVA model.

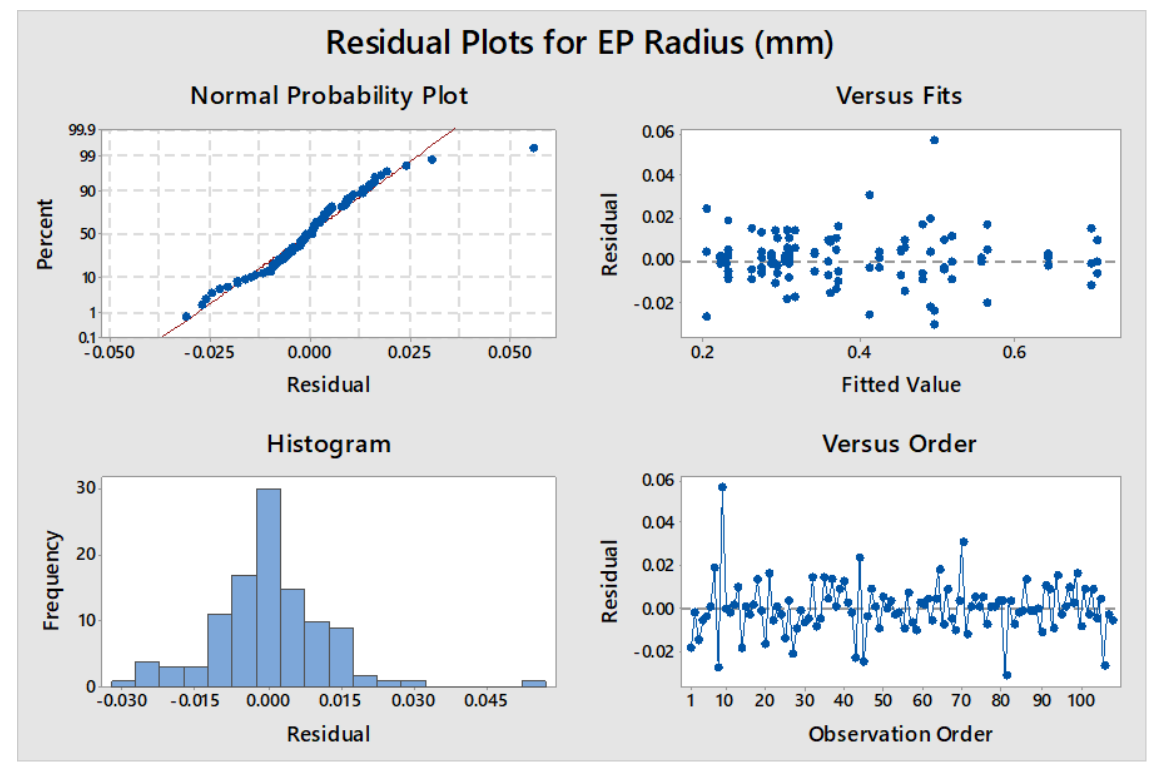

Figure 56: Four-in-one plot for electropolished radius measurements.

There is nothing unusual about the four-in-one plot of the residuals for this ANOVA model; there is not a significant departure from normality, there is no trend in the residual versus order graph, and there is a similar variance. As a separate measurement, an ANOVA test was performed on the measured angle of each corner. The ANOVA table can be seen in Figure 57.

Analysis of Variance

\begin{tabular}{lrrrrr} 
Source & DF & Adj SS & Adj MS & F-Value & P-Value \\
\hline hole & 8 & 143.24 & 17.905 & 26.78 & 0.000 \\
corner & 3 & 332.31 & 110.770 & 165.65 & 0.000 \\
hole*corner & 24 & 512.25 & 21.344 & 31.92 & 0.000 \\
Error & 72 & 48.14 & 0.669 & & \\
Total & 107 & 1035.94 & & &
\end{tabular}

Figure 57: ANOVA output for electropolished angle measurements, showing significance of hole and corner interaction. 
Similar to the radius measurements, the interaction of hole and corner was significant. Therefore, it can be inferred that changing the hole and corner affected the amount of material removed. As more material was removed at the corner, the measured angle became higher. The four-in-one plots, as shown in Figure 58, indicates some violations of the assumptions of ANOVA.

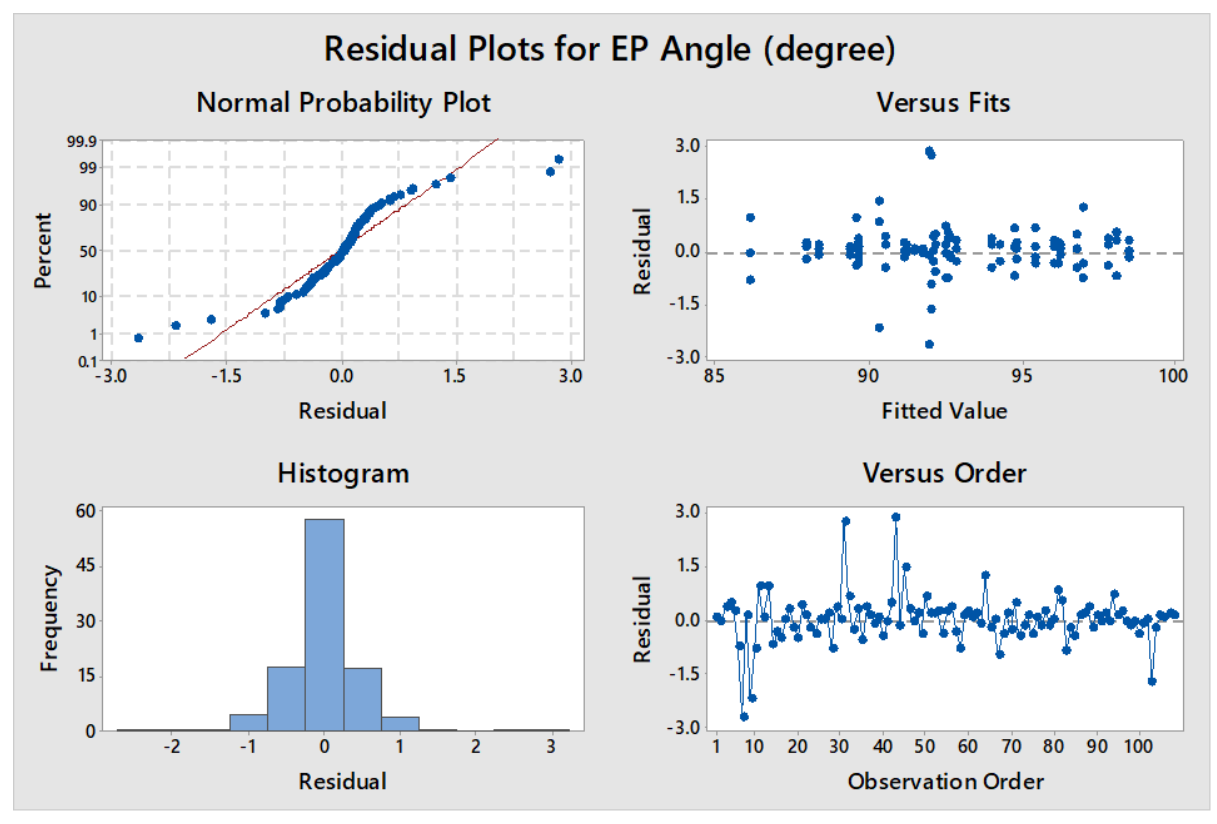

Figure 58: Four-in-one plot for electropolished angle measurements.

The normal probability plot has a slight 's' shape to it, indicating a non-normal residual, violating the assumption of normality required for ANOVA. However, ANOVA is fairly robust when dealing with non-normality, so this was considered a non-issue. The residuals versus fitted values and residuals versus observation order were not unusual. Due to the unreliability of the angle and radius measurements of the as-printed sample, statistical analysis was not performed. However, a more reliable measurement was the perpendicular distance from edge to edge across all of the holes. Four measurements per hole were made. Due to the failure of the control sample during printing, only six of the 
nine holes were available for comparison. Furthermore, only the standard size of hole was compared, leaving four holes. To compare the results, a two-sample t-test was performed. The hypothesis and results are shown in Figure 59.

\section{Method}

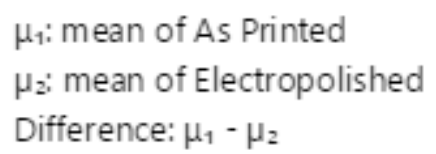

Test

\begin{tabular}{llr} 
Null hypothesis & $\mathrm{H}_{0}: \mu_{1}-\mu_{2}=0$ \\
Alternative hypothesis & $\mathrm{H}_{1}: \mu_{1}-\mu_{2}<0$ \\
T-Value & DF & P-Value \\
\hline-1.59 & 30 & 0.061
\end{tabular}

Figure 59: Hypothesis and test results for two-sample t-test for the difference in means between electropolished and as-printed hole distances, showing significance.

Although the $\mathrm{P}$-value is above the standard accepted $\mathrm{P}=0.05$ level, this result was still considered significant, as the measurements had high precision and reliability. The difference in means was small, as can be seen in Figure 60.

\section{Descriptive Statistics}

\begin{tabular}{lrrrr} 
Sample & N & Mean & StDev & SE Mean \\
\hline As Printed & 16 & 6.5015 & 0.0896 & 0.022 \\
Electropolished & 16 & 6.5450 & 0.0628 & 0.016
\end{tabular}

Figure 60: Descriptive statistics of as-printed and electropolishing distance measurements. Units in millimeters.

The differences in mean is small, but the precision of the measurements performed by the CMM allow for a high level of confidence in the accuracy of those measurements. The accuracy of the measurements can be seen in the miniscule standard deviation.

Additionally, when considering precision applications, a difference of $0.04 \mathrm{~mm}$ can make a large difference in tolerancing a part. 


\subsubsection{Surface Roughness}

Surface roughness measurements were performed on the entrances to all holes and recorded on a per-edge basis, as seen in Figure 61.
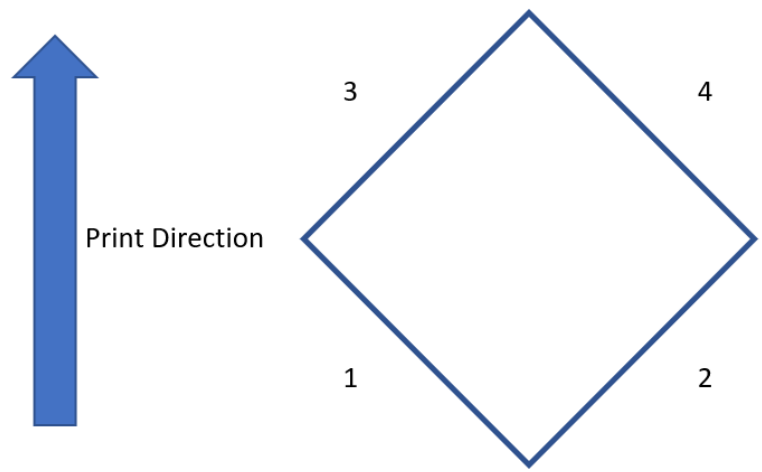

Figure 61: Labeling convention for surfaces measured by profilometer.

The labeling started with the bottom left surface, increasing counter-clockwise. Three measurements were performed for each surface to ensure accuracy. A boxplot breaking the measured surface roughness values by edge is shown in Figure 62 .

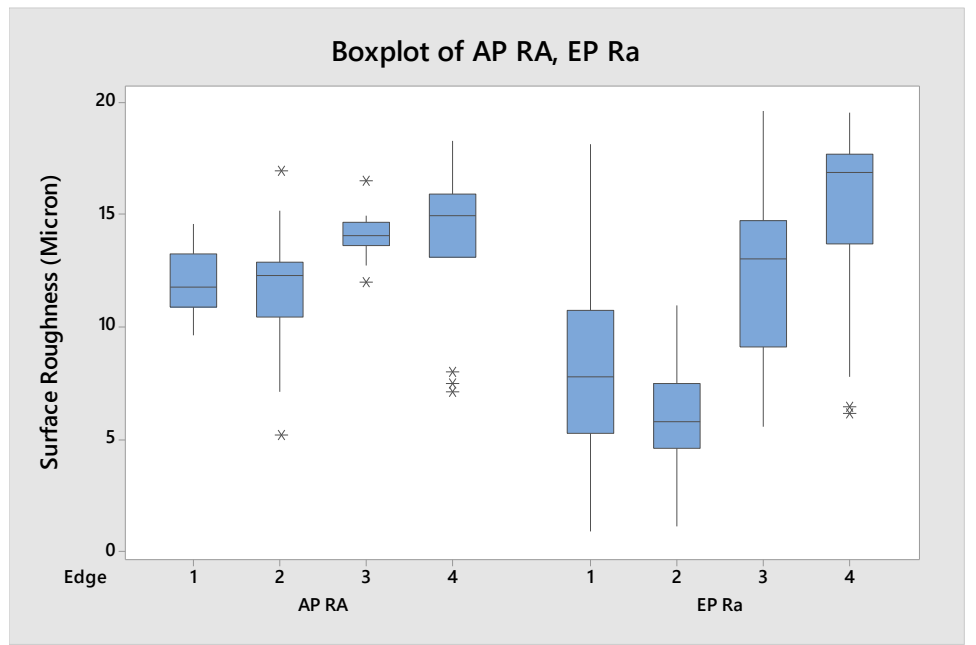

Figure 62: Boxplot comparing average measured surface roughness of as printed (AP) and electropolished (EP) holes, broken down by specific edge. 
A general trend is visible on the graph; for the as-printed hexagon, the pair of $45^{\circ}$ upward facing surfaces had approximately the same surface roughness, with a significant increase in average surface roughness for the pair of downward facing surfaces. However, there was a slightly different trend for the electropolished sample, which saw a decrease in average surface roughness between both upward and downward facing pairs. An analysis of variance test was performed on the measured average surface roughness values, using hole and edge as factors. The results can be seen in Figure 63.

\begin{tabular}{lrrrrr}
$\begin{array}{l}\text { Analysis of Variance } \\
\text { Source }\end{array}$ & DF & Adj SS & Adj MS & F-Value & P-Value \\
\hline Hole & 8 & 485.1 & 60.637 & 14.95 & 0.000 \\
Edge & 3 & 1746.9 & 582.309 & 143.59 & 0.000 \\
Hole*Edge & 24 & 308.2 & 12.843 & 3.17 & 0.000 \\
Error & 72 & 292.0 & 4.055 & & \\
Total & 107 & 2832.2 & & &
\end{tabular}

Figure 63: ANOVA table for average measured surface roughness of the electropolished hexagon, using hole and edge as factors. The interaction between hole and edge was found to be significant.

The interaction between hole and edge was found to be significant, meaning there is a significant difference in how the measured surface roughness changes based on where on the hexagon it is. These results are consistent with expectations; the different designs of the holes in the hexagon allow for different amounts of polishing to occur, while the difference in surface roughness by edge causes a large difference too. A four-in-one plot verifying the assumptions of this ANOVA model can be seen in Figure 64. 


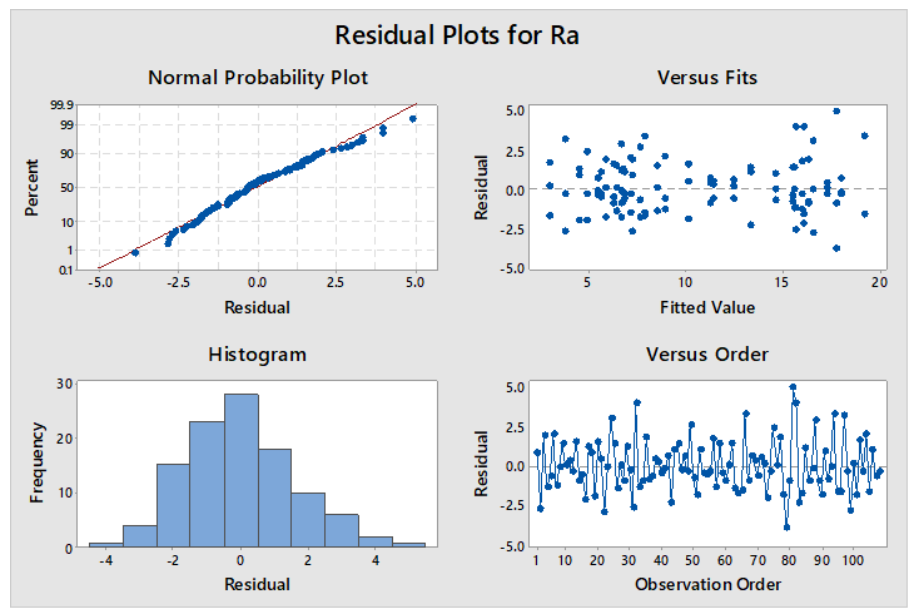

Figure 64: Four-in-one residual plot for measured surface roughness of electropolished hexagon.

There is nothing unusual about the plots of the residuals versus the observation order or the residuals versus the fitted values. There is a slight deviation from normality, but it is not large enough to affect the integrity of the ANOVA model. To better understand the results of the ANOVA model, an interaction plot of hole and edge was constructed, shown in Figure 65.

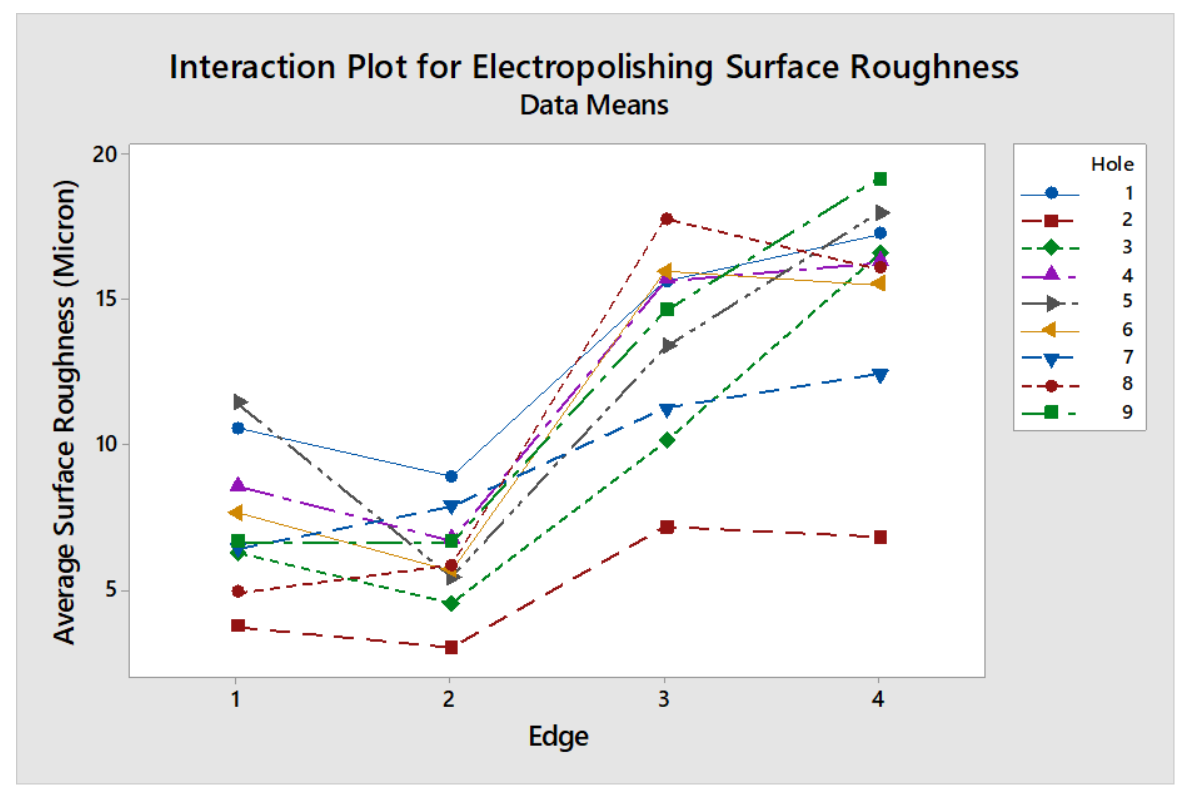

Figure 65: Interaction plot of hole and corner for the electropolished hexagon. 
The trend from the boxplot of surface roughness measurements by edge is clearly seen in the interaction plot. However, the type of hole also significantly influenced the change in surface roughness. The descriptive statistics for the mean surface roughness by hole can be seen in Figure 66.

\section{Statistics}

\begin{tabular}{llrrrrr} 
Variable & Hole & Mean & SE Mean & StDev & Minimum & Maximum \\
\hline $\mathrm{Ra}$ & 1 & 12.24 & 1.35 & 4.68 & 4.49 & 17.61 \\
& 2 & 5.157 & 0.742 & 2.572 & 0.910 & 9.120 \\
& 3 & 9.35 & 1.48 & 5.14 & 2.40 & 19.55 \\
& 4 & 11.79 & 1.38 & 4.77 & 5.79 & 19.61 \\
& 5 & 12.04 & 1.39 & 4.80 & 5.00 & 18.63 \\
& 6 & 11.19 & 1.45 & 5.01 & 5.05 & 17.72 \\
& 7 & 9.495 & 0.845 & 2.927 & 4.960 & 13.000 \\
& 8 & 11.14 & 1.92 & 6.66 & 2.84 & 22.67 \\
& 9 & 11.76 & 1.70 & 5.88 & 4.77 & 22.48
\end{tabular}

Figure 66: Descriptive statistics for average surface roughness by hole for the electropolished hexagon.

In general, the holes had similar minimum and maximum measured surface roughness.

Of note is Hole 2, which had a much lower measured surface roughness than all other holes, this is not necessarily due to better electropolishing; the edge of the hole was too short to be measured using the same cut-off length on the profilometer as the other holes, so it was measured using a shorter cut-off length, which tends to produce smaller roughness values. However, it was found that the inclusion of these values did not change the outcome of the ANOVA model, so it was left in the model. Another interesting note is the variance and similarities in measured means. Hole 4 and Hole 7 were replicates of each other, yet Hole 7 had an average surface roughness $2 \mu \mathrm{m}$ lower than Hole 4. Some holes experienced a large drop in surface roughness between upward facing surfaces, 
with a similar difference in downward facing surfaces. Other holes experienced less significant changes between the different edges. A multiple comparison test was performed, as seen in Figure 67.

\section{Grouping Information Using the Tukey Method and 95\% Confidence}

\begin{tabular}{lrrrrr} 
Corner & N & Mean & \multicolumn{2}{l}{ Grouping } \\
\hline 4 & 27 & 15.3400 & A & & \\
3 & 27 & 13.4970 & & B & \\
1 & 27 & 7.3344 & & C \\
2 & 27 & 6.0507 & & C
\end{tabular}

Means that do not share a letter are significantly different.

Figure 67: Multiple comparisons for the mean surface roughness by edge.

There is no significant difference between the upward facing surfaces, but there is a significant difference in the downward facing surfaces. This is most likely due to there being more excess material on the downward facing surfaces than the upward facing surfaces, meaning that it was easier to for the polishing to remove. Additionally, this means that polishing orientation did have a significant effect on how the surfaces were polished. An ANOVA model was constructed for the same factors, this time for the asprinted interior surface roughness, shown in Figure 68.

\begin{tabular}{lrrrrr}
$\begin{array}{l}\text { Analysis of Variance } \\
\text { Source }\end{array}$ & DF & Adj SS & Adj MS & F-Value & P-Value \\
\hline hole & 3 & 3.477 & 1.159 & 0.68 & 0.572 \\
edge & 3 & 84.177 & 28.059 & 16.39 & 0.000 \\
hole*edge & 9 & 37.269 & 4.141 & 2.42 & 0.032 \\
Error & 32 & 54.773 & 1.712 & & \\
Total & 47 & 179.697 & & &
\end{tabular}

Figure 68: ANOVA table for surface roughness measurements of as-printed interior surfaces; the interaction of hole and edge was found to be significant. 
Interestingly, the interaction of hole and edge was found to be significant for the asprinted as well. Due to the large significance of the edge term, it would appear that it dominates the interaction, and is seemingly providing significance when there is not actually any. The residual plots for this ANOVA model are seen in Figure 69.

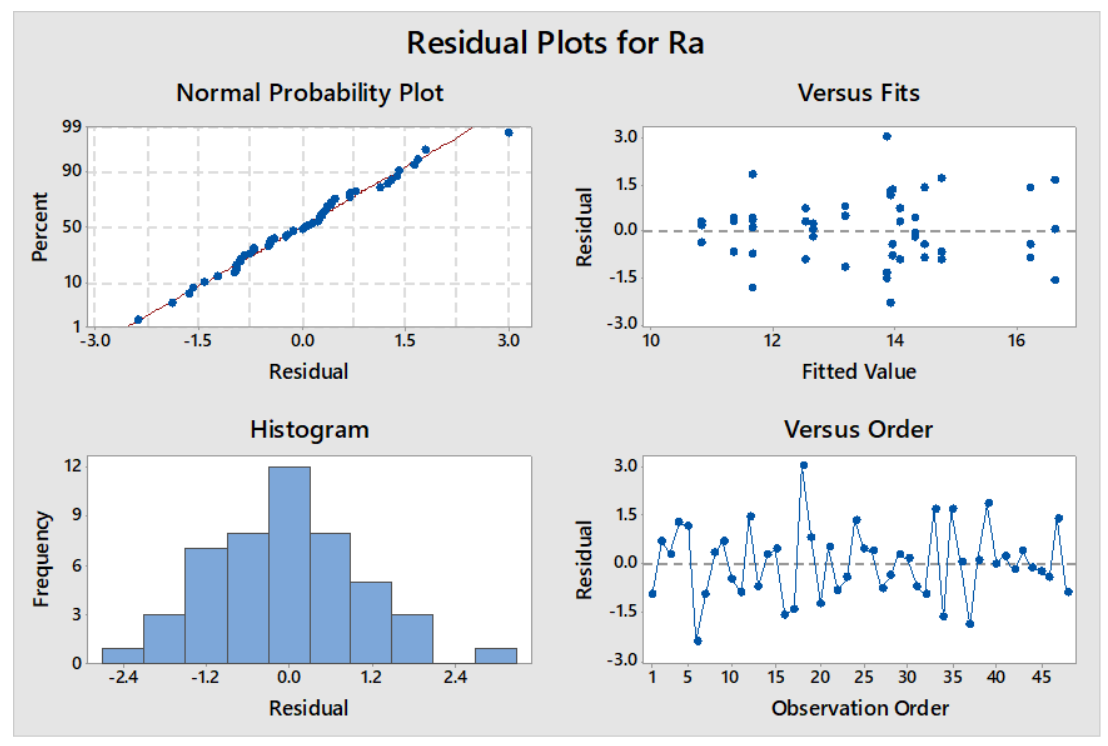

Figure 69: Residual plots for the average surface roughness measurements of as-printed hexagon; no indication of deviation from ANOVA assumptions.

There is nothing unusual about the residual plots. During measurements, some surfaces were too small to be measured using the longer cut-off length used in the majority of measurements. Unlike the electropolished sample, these measurements were taken as outliers by the ANOVA model and were removed from this analysis. The interaction plot for this ANOVA model can be seen in Figure 70. 


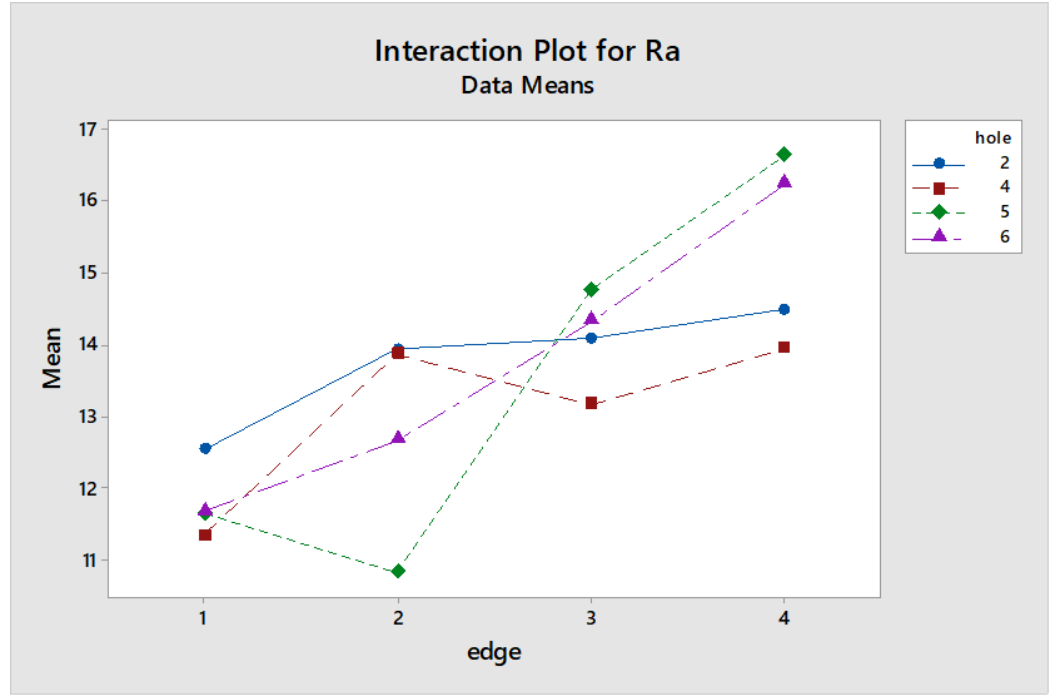

Figure 70: Interaction plot for measured surface roughness of as-printed part.

This data appears to suggest the same trend as the electropolished interior surface roughness. However, it is possible that the data highlights the inaccuracy of the profilometer; even with multiple measurements per surface, it is possible to choose rougher surfaces each time, which would strongly influence the results. Finally, ANOVA was conducted on the measured surface roughness for the outside surfaces. Due to the presence of support structure stubs, only eight surfaces were available for measurement. Of these eight surfaces, there were only four unique surfaces were available for analysis: the $0^{\circ}, 30^{\circ}$, and $60^{\circ}$ upward facing surfaces and the $90^{\circ}$ surface. The factors considered in this analysis were whether the part had been processed by electropolishing, and which surface was being measured. The results of the analysis are in Figure 71. 


\section{Analysis of Variance}

\begin{tabular}{lrrrrr} 
Source & DF & Adj SS & Adj MS & F-Value & P-Value \\
\hline Surface & 3 & 70.722 & 23.574 & 11.31 & 0.000 \\
Processed & 1 & 234.156 & 234.156 & 112.37 & 0.000 \\
Surface*Processed & 3 & 6.412 & 2.137 & 1.03 & 0.393 \\
Error & 34 & 70.849 & 2.084 & & \\
Total & 41 & 387.987 & & &
\end{tabular}

Figure 71: ANOVA results for measured surface roughness, considering surface and processing history as factors. Both factors were independently significant, but the interaction was not significant.

The insignificance of the interaction supports the evidence of the line of sight nature of the electropolishing process. All the surfaces reduced their surface roughness from electropolishing, but it did not matter which surface it was. Additionally, the measured surface had a significant effect on the surface roughness without respect to whether the part was processed or not, due to the varying as-printed surface roughness. The residual plot for the ANOVA model can be seen in Figure 72.

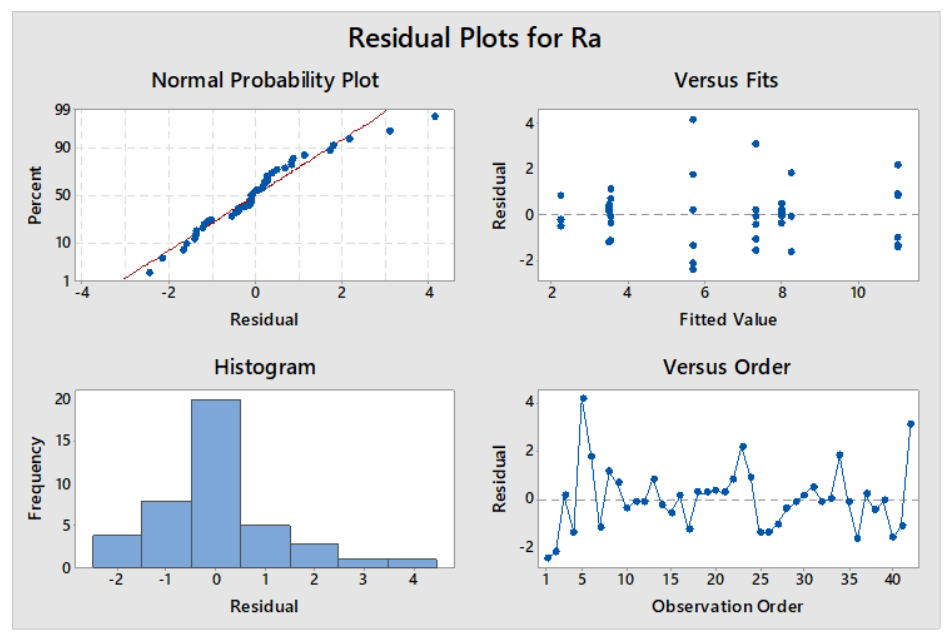

Figure 72: Residual plots for ANOVA model of exterior surface roughness measurements, indicating no violations of assumptions of ANOVA. 
There is nothing unusual about the residual plots, nor is there any significant deviation from normality. The Tukey method was used to perform multiple comparisons for the measured surface roughness of the exterior surfaces, shown in

\section{Grouping Information Using the Tukey Method and 95\% Confidence}

\begin{tabular}{lrrr} 
Surface & N & Mean & Grouping \\
\hline 1 & 12 & 8.34917 & A \\
2 & 12 & 5.76750 & \\
4 & 12 & 5.39250 & B \\
3 & 6 & 5.21500 & B
\end{tabular}

Means that do not share a letter are significantly different.

Figure 73: Multiple comparisons for the four unique exterior surfaces measured average surface roughness.

The multiple comparisons show that the average measured surface roughness was highest on surface 1 , which corresponds to the $30^{\circ}$ upwards facing surface. The other surfaces had significantly lower surface roughness than surface 1 but were not significantly different from each other. This may be due to the protruding corner of the $60^{\circ}$ upward facing surface blocking part of the other surface from being in direct line of sight during the polishing process, reducing the amount of polishing it received. Finally, a multiple comparison test was performed between the processed and as-printed sample, shown in

\section{Grouping Information Using the Tukey Method and 95\% Confidence}

\begin{tabular}{lrrr} 
Processed & N & Mean & Grouping \\
\hline 2 & 21 & 8.65042 & A \\
1 & 21 & 3.71167 & B \\
& & &
\end{tabular}

Figure 74: Multiple comparisons test for electropolished and as-printed measured surface roughness for all surfaces. Electropolished is shown as '1' and as-printed as '2'. 
Electropolishing improved the overall surface roughness of the exterior by $5 \mu \mathrm{m}$. This is a significant improvement over the as-printed state, but still does not match the surface roughness values achieved in literature and is still not low enough to be considered applicable for engineering application.

\subsubsection{Laser Polishing}

Laser polishing was carried out by the company Laser Light Technologies in Hermann, Missouri. Overall, the surface produced was smoother than the as-printed, but the laser polishing was not carried out in a vacuum or shielding gas, producing a blackened surface.

\subsubsection{Imaging}

To better understand the results of the laser polishing process, a series of images were taken. Figure 75 shows the two sides of the coupon that were processed.

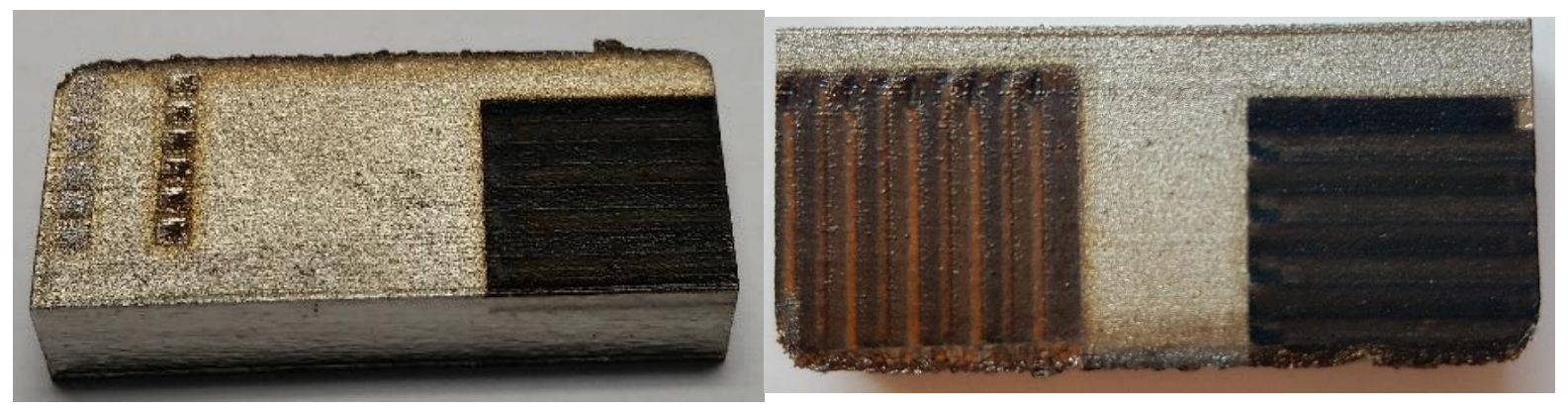

Figure 75: Laser polishing sample as-received (Left) front side of sample (Right) back side of sample. 
The twelve small squares are broken down into two sets of six; the leftmost set of six is the result of the $1 \mathrm{~J} / \mathrm{cm}^{2}$ energy density, while the other set of six is the results of the 4 $\mathrm{J} / \mathrm{cm}^{2}$ energy density. These energy densities were determined to be good starting points for the evaluation of the process by the polishing company, with the idea that the energy densities could be increased following initial evaluation; however, due to time constraints, only these energy densities were able to be tested in this study. The amount of pulses from the laser was increased per each square. Figure 76 shows two subsequent squares resulting from the $4 \mathrm{~J} / \mathrm{cm}^{2}$ energy density.

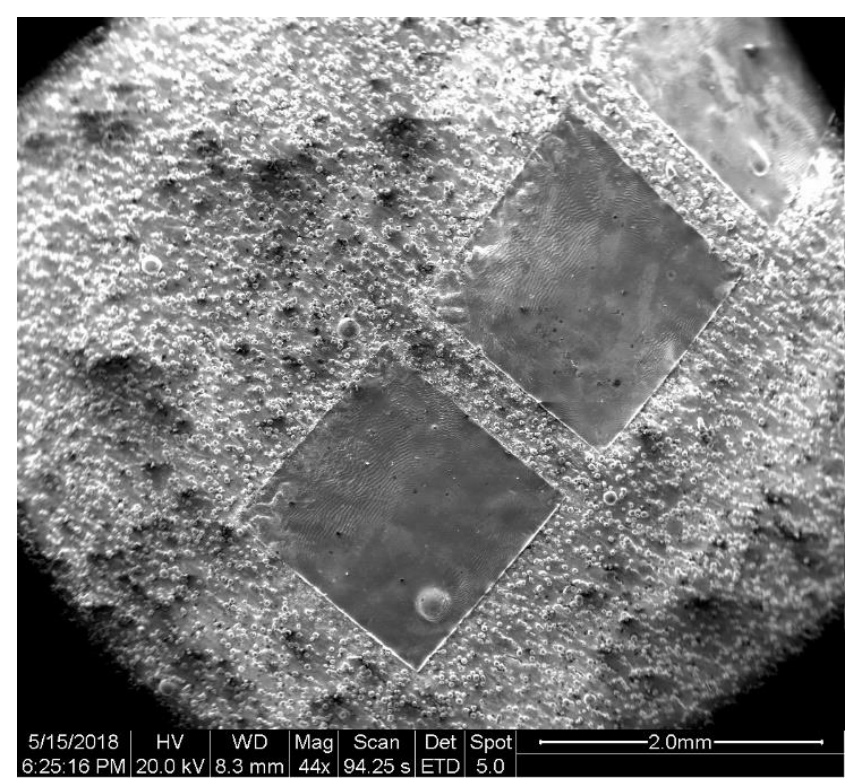

Figure 76: 44x magnification SEM image showing two $4 \mathrm{~J} / \mathrm{cm}^{2}$ polishing squares contrasted with the bulk material.

The large, striped squares are the results of different scanning strategies resulting from the optimal parameters chosen from the twelve square test. Due to the large amount of blackened area, it can be assumed that the laser polishing was not performed in the presence of a shielding gas or under a vacuum, therefore allowing a large amount of 
oxidation to occur. Initial SEM imaging was done on the $4 \mathrm{~J} / \mathrm{cm}^{2}$ energy density squares, as seen in Figure 77.

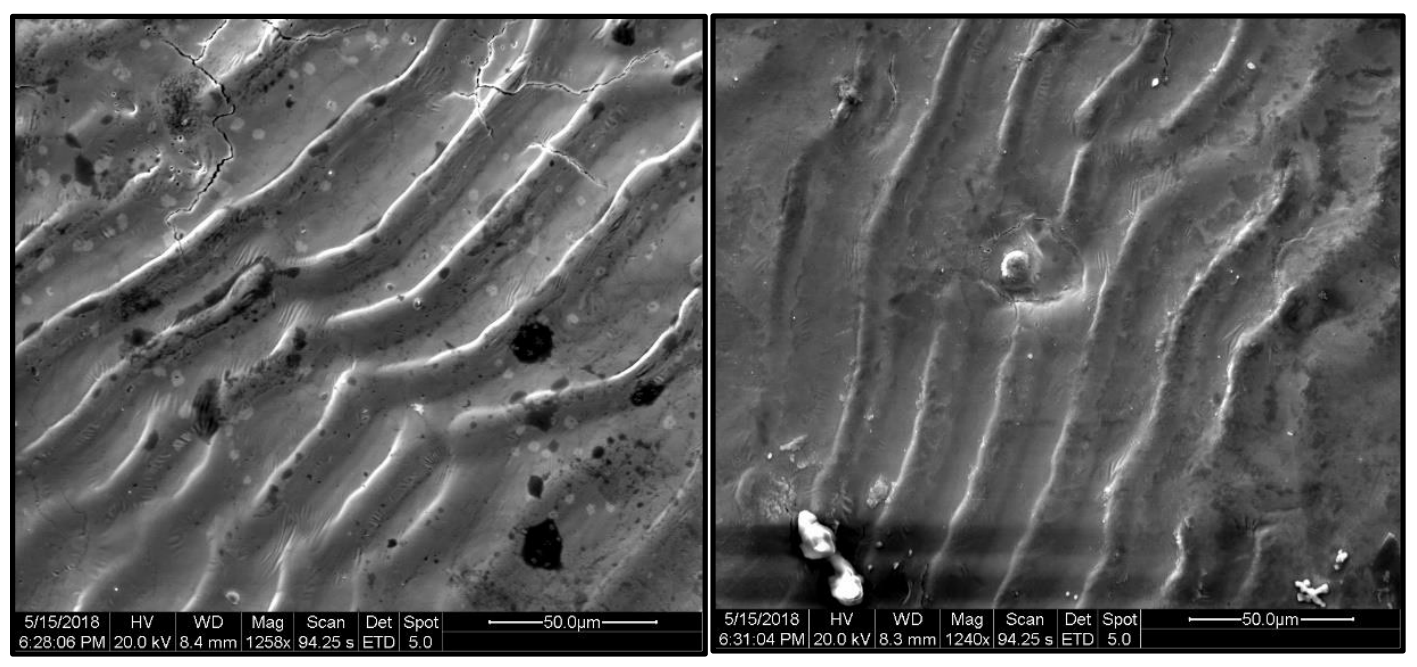

Figure 77: SEM images of $4 \mathrm{~J} / \mathrm{cm}^{2}$ test squares showing ripples typical of resolidification process immediately following laser polishing. (Left) 1258x image of bottom-most polishing square containing significant cracking. (Right) 1240x image of second from the bottom polishing square showing powder particle warping ripple formation.

The ripples formed are indicative of re-solidification of surface melted material. As the metal cools rapidly, surface tension gradients form, pulling the molten material into the stripes seen. Additionally, due to the polishing in air, an oxide layer formed and subsequently cracked upon fully cooling. SEM images of the $1 \mathrm{~J} / \mathrm{cm}^{2}$ test square showed little effect on the material, such as in Figure 78. 


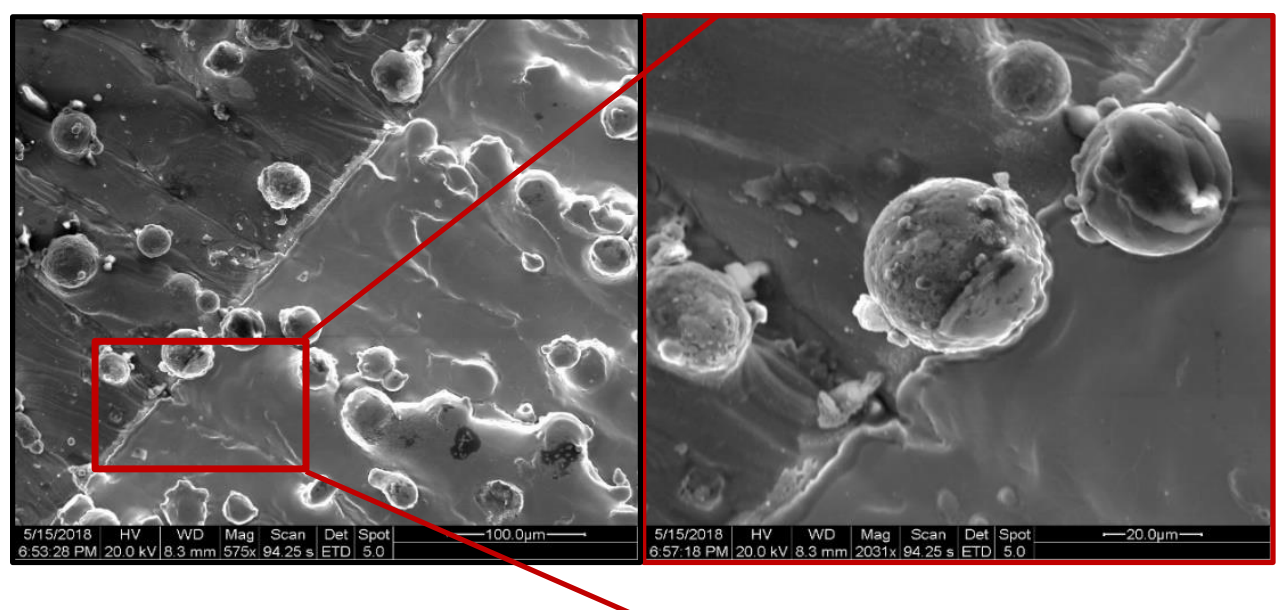

Figure 78: SEM images of border of $1 \mathrm{Kcm}^{2}$ test square showing the inability to deal with large parasitic powder particles; the un-polished metal is darker.

The $1 \mathrm{~J} / \mathrm{cm}^{2}$ energy density was too low to significantly affect the surface of the metal; as seen in the left image, the polishing process was only able to polish the smallest defects. In fact, it was only able to polish the surface of a $\sim 30 \mu \mathrm{m}$ diameter parasitic powder particle. Many of the surface features of the as-printed material remained following the 1 $\mathrm{J} / \mathrm{cm}^{2}$ energy density polishing, albeit slightly muted. However, the $4 \mathrm{~J} / \mathrm{cm}^{2}$ energy density, when scanned in tracks, was able to produce its own surface features, as seen in Figure 79.

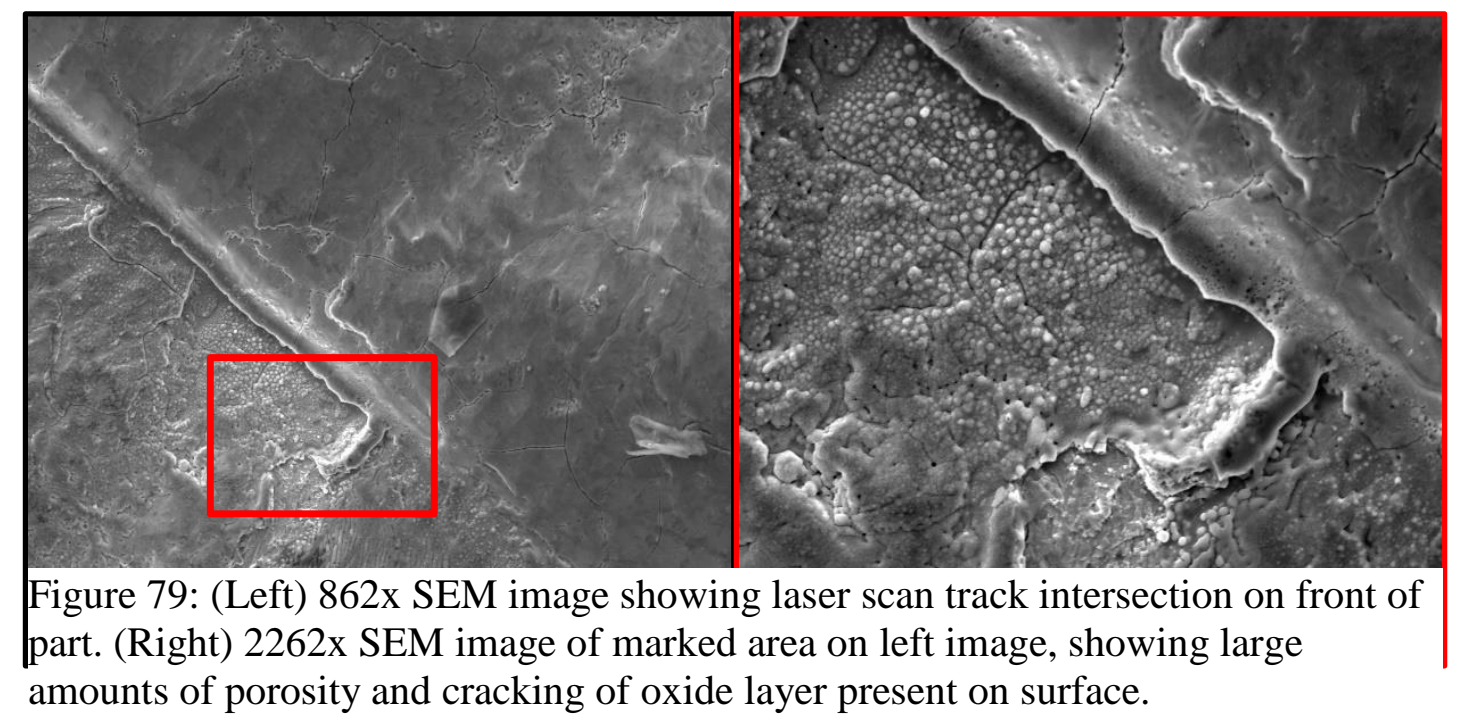


The laser scan tracks were highly visible on the part. The overlap of the scan tracks produced ripples that appeared to form solidification waves over nearby metal; they solidified above the underlying material, producing a gap. Additionally, there was an increase in visible cracking at the overlaps of the scan tracks. At the higher energy density, a much smoother surface was produced. However, this energy density was still not high to fully remove all parasitic powder particles, as seen in Figure 80.
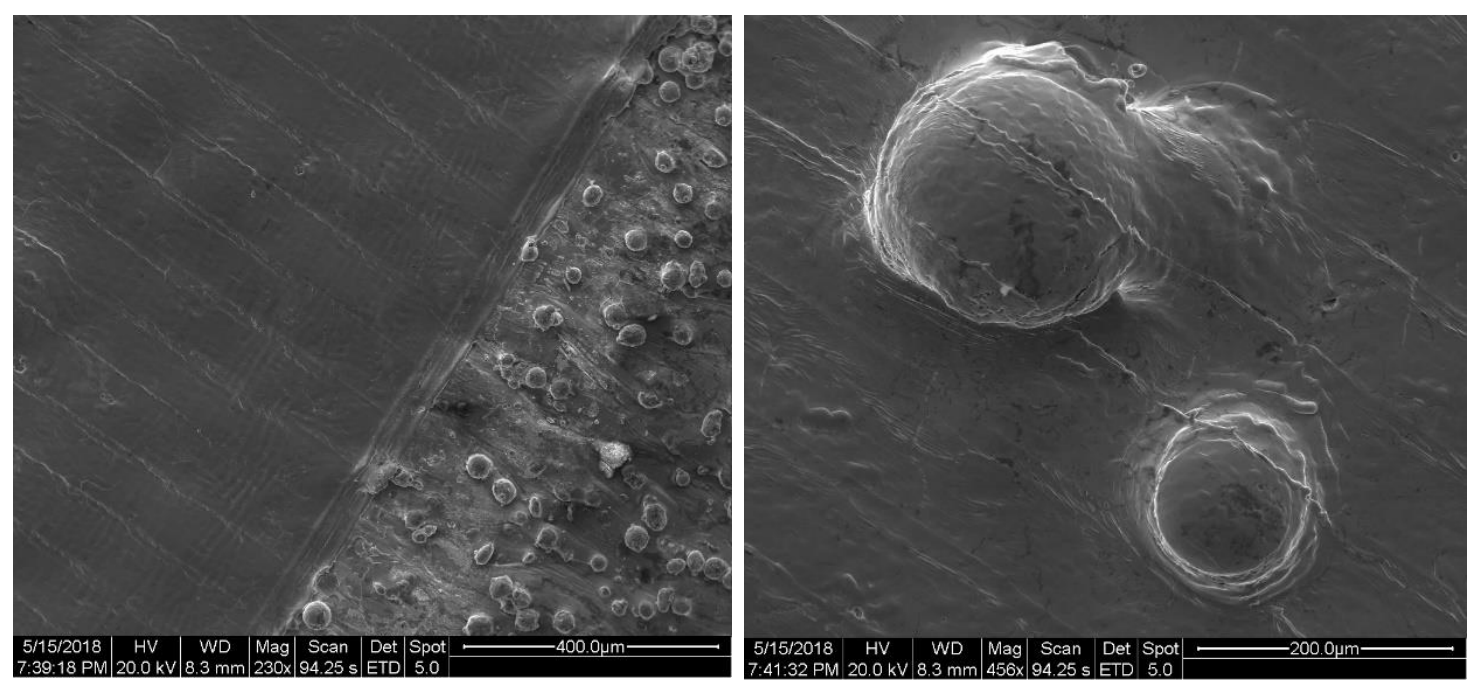

Figure 80: (Left) 230x magnification SEM image of laser polishing on back of sample. (Right) 456x SEM image of parasitic powder particles with clear laser scan marks.

At the border between as-printed material and the laser polished material, it is clear that there has been an improvement in the surface finish. However, underlying remnants of previous structures can still be seen. While there is much less warpage of scan tracks around these particles, they are still visible. Additionally, the characteristic ripples were still present, forming perpendicular to the scanning direction. Large particles still presented a challenge to the laser polishing process as well; these particles were so large that the remained unperturbed on the surface, with only a small amount of material being melted at the surface and reallocated. 
To evaluate the effects on laser polishing on the microstructure, metallography was performed on the surface of the laser polished sample, as well as on the cross-section. The potential heat affected zone was examined first, seen in Figure 81.

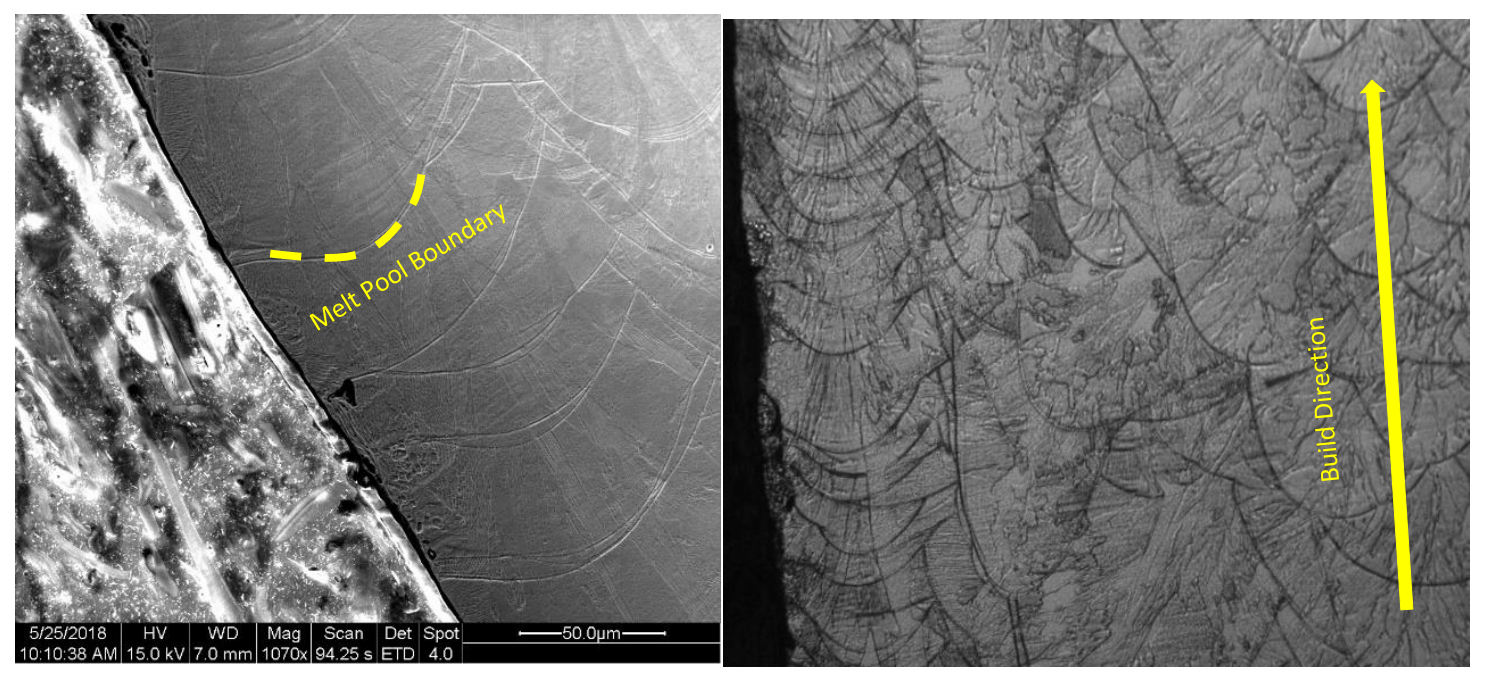

Figure 81: (Left) 1070x SEM image of potential heat-affected zone edge. (Right) 500x optical microscope image of potential heat affected zone microstructure.

The microstructure seen is characteristic of the as-built microstructure. The narrower size of the melt pools on the left-most edge of the sample is due to a machine setting for the laser that reduces power to prevent unwanted melting of adjacent powder particles. At these magnifications, there is no clear evidence of any heat affected zone. The magnification was increased to look for more evidence of a significant heat affected zone, shown in Figure 82. 


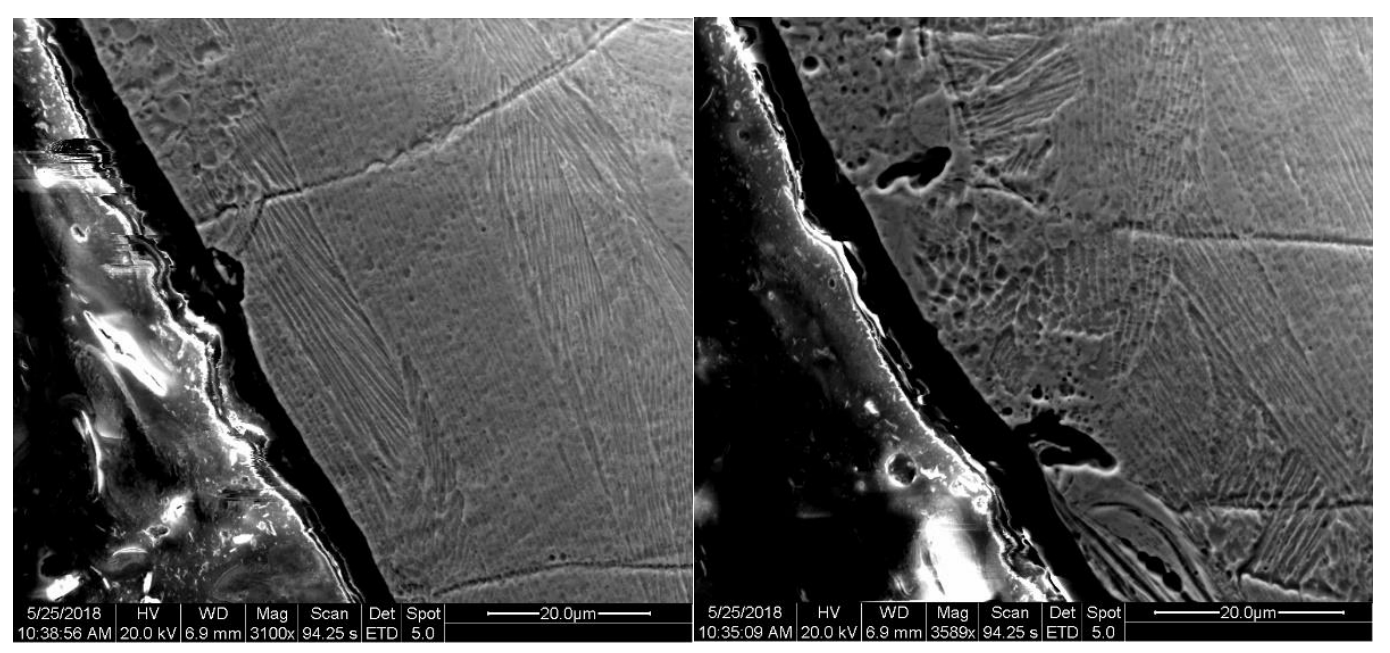

Figure 82: SEM images of laser polished edge showing no sign of heat affected zone, but does show some porosity.

The microstructure in these images show no sign of any difference from the base material. There does appear to be some dimples, which may be a result of tear-out from the cutting saw and not from the laser polishing process. Additionally, there is some porosity visible in the right image. While this is consistent with literature, it is unclear whether this is due to the metallography process or a result of the laser polishing process. The dimples immediately adjacent to the apparent porosity suggests that the tear-out caused by the cut-off saw may have caused some material removal that was then filled by bakelite.

After the heat affected zone was investigated, the microstructure of the surface was imaged. The laser polishing occurred on the $90^{\circ}$ face of the part, resulting in a microstructure with the layers clearly visible, such as in Figure 83. 


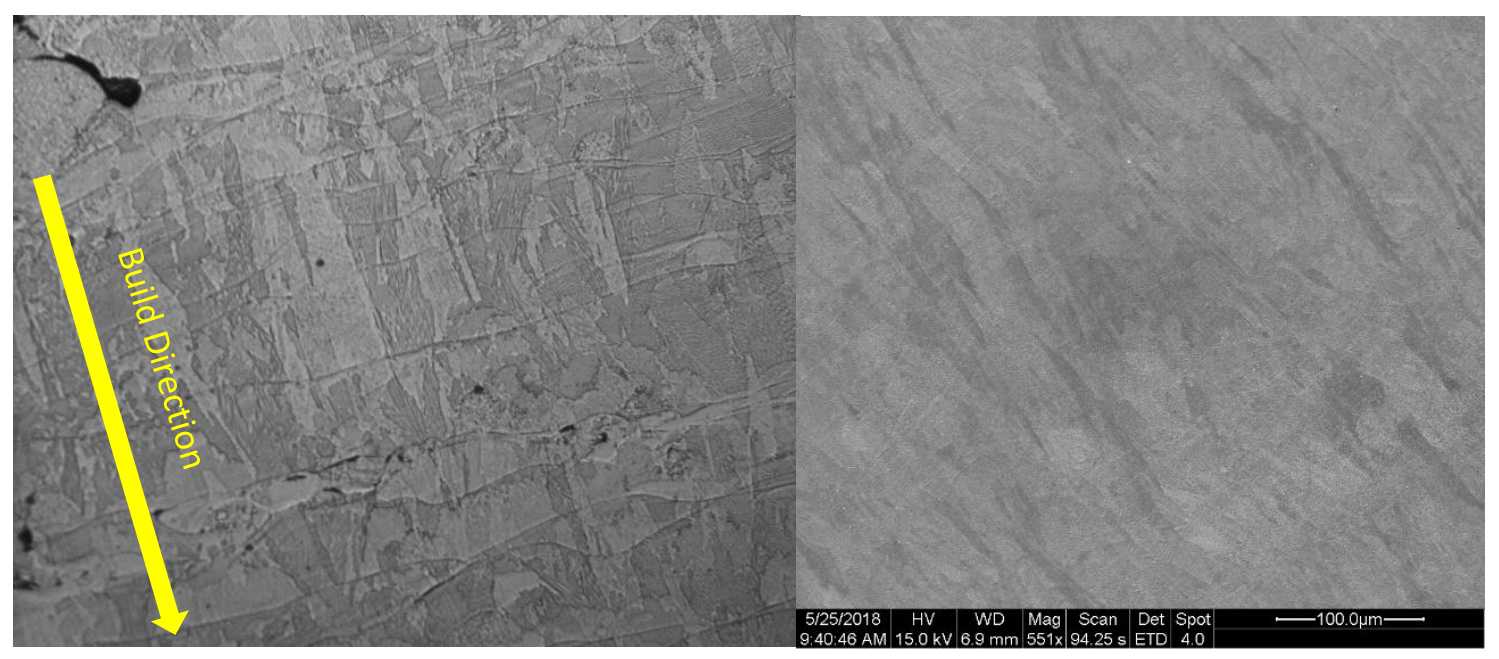

Figure 83: (Left) 200x, optical microscope image of microstructure. (Right) 551x SEM image of microstructure.

Again, at low magnification there is no significant evidence of a change in microstructure from the as-printed state. The microstructure in the previous figure shows clear evidence of the characteristic layer structure, with defined melt pool boundaries and columnar growth in the build direction. Imaging was performed at a higher magnification to observe sub-grain structure, shown in Figure 84.

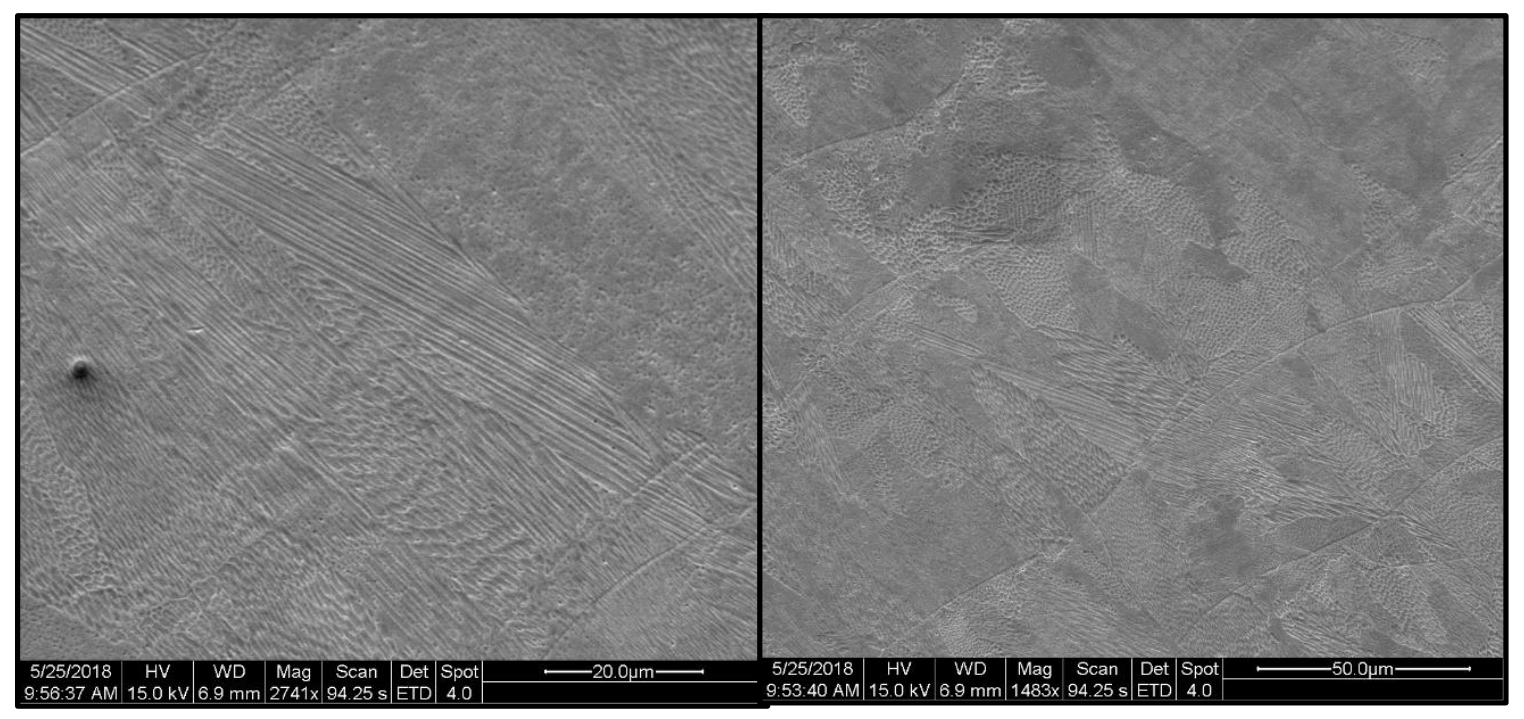

Figure 84: SEM images of etched laser polished sample, showing no significant change in microstructure as a result of laser polishing. 
At this magnification, the sub-grain structure is visible. Both the characteristic cellular and columnar microstructures are evident. The columnar sub-grains are preferentially aligned with the melt pool boundaries. These features are characteristic of the as-printed microstructure, meaning there is no evidence of a change in microstructure due to laser polishing.

\subsubsection{Surface Roughness}

The company, Laser Light Technologies, provided laser profilometer images for a variety of polishing applications. The laser profilometer produces a topographical map for the surface roughness of the scanned area. The first scan, seen in Figure 85, shows a characteristic image of the as-printed coupon.

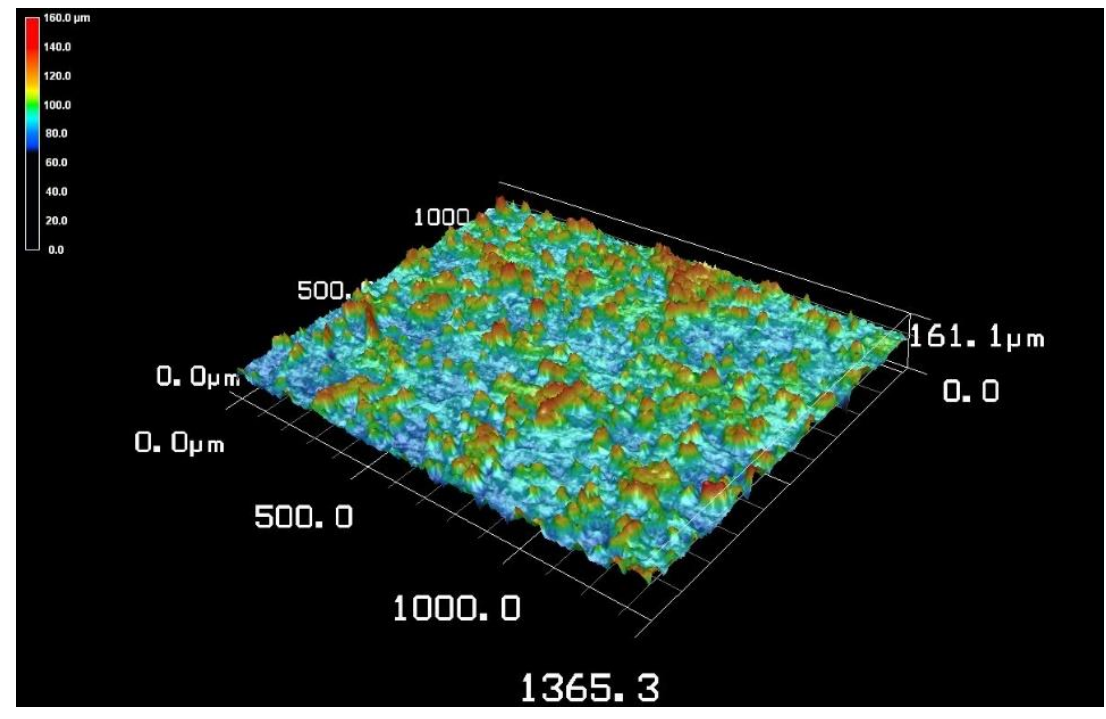

Figure 85: Laser profilometer measurement of as-received coupon.

The scanned area shows that the highest peak was $161.1 \mu \mathrm{m}$ from the lowest valley. The measured surface roughness for this surface was $7.2 \mu \mathrm{m}$. This is slightly lower than the surface roughness values obtained from the mechanical tip profilometer. The first test run 
was at an energy density of $1 \mathrm{~J} / \mathrm{cm}^{2}$ with 50 pulses. The resulting surface is seen in Figure 86.

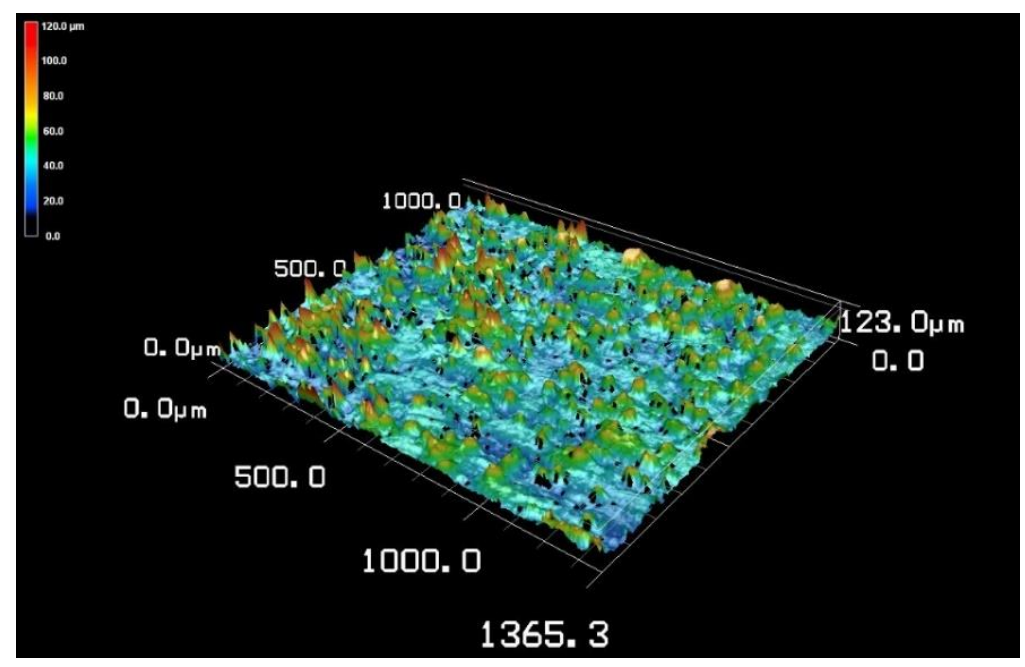

Figure 86: Laser profilometer measurement of area exposed to $1 \mathrm{~J} / \mathrm{cm}^{2}$ energy density and 50 pulses.

While the highest peak of the surface appears to have diminished, there is largely no change in the surface immediately following the treatment. A large number of peaks still remain, and the surface looks relatively unchanged compared to the as-printed state. While maintaining the same energy density, the number of pulses was increased 
inintervals of 25 . There was largely no difference in the surface produced until 175 pulses was reached. The resulting surface can be seen in Figure 87.

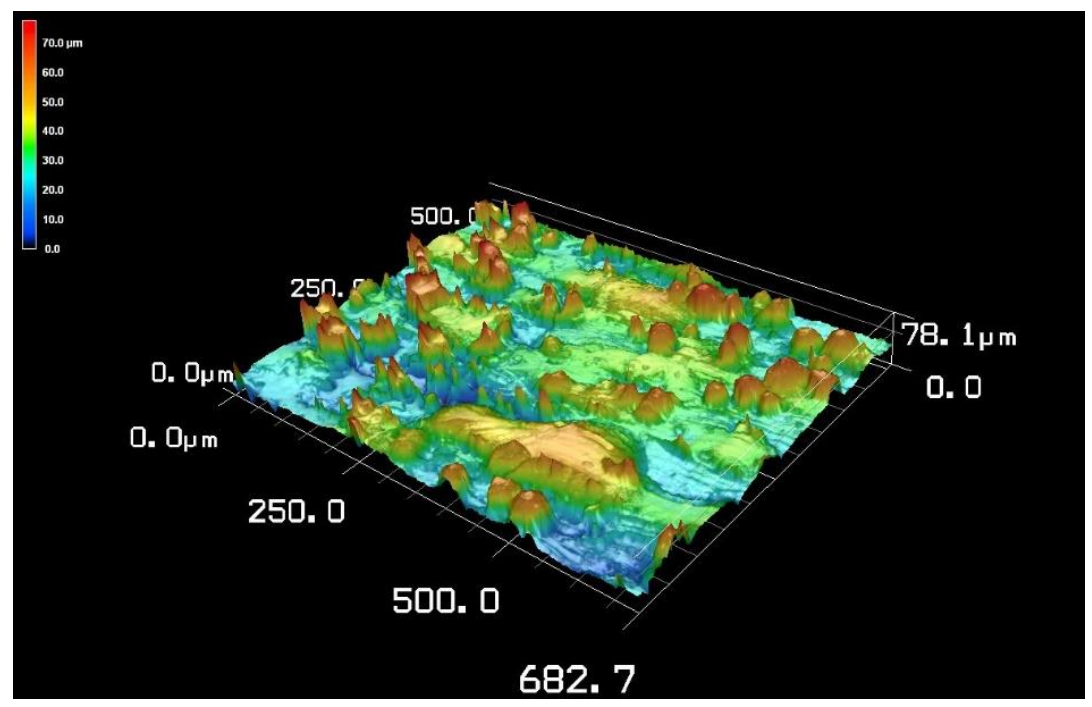

Figure 87: Laser profilometer measurement of area exposed to $1 \mathrm{~J} / \mathrm{cm}^{2}$ energy density and 175 pulses.

The laser profilometer scan shows that after 175 pulses of $1 \mathrm{~J} / \mathrm{cm}^{2}$ energy, the measured Rz has decreased by approximately $50 \mu \mathrm{m}$. However, it is still clear that the previous surface remains largely intact. There is a large number of peaks and other surface roughness artifacts still present on the surface. While it is an improvement over the original surface, it is certainly not a large enough improvement to consider the $1 \mathrm{~J} / \mathrm{cm}^{2}$ energy density useful. However, the laser profilometer data seen in Figure 88 of the larger energy density indicates an instant improvement when upping the energy density. 


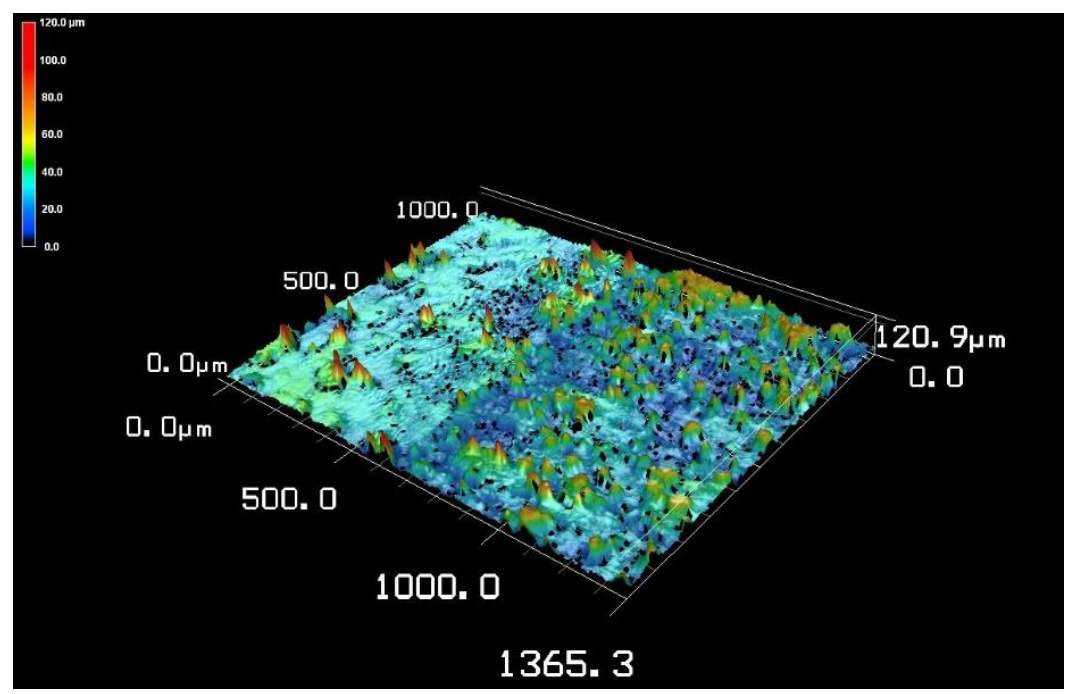

Figure 88: Laser profilometer measurement of area exposed to $4 \mathrm{~J} / \mathrm{cm}^{2}$ energy density and 75 pulses.

This scan is directly on the border between the area exposed to the $4 \mathrm{~J} / \mathrm{cm}^{2}$ energy density and the as-printed material. The direct comparison shows that while this energy density was able to polish the surface of the smaller particles, large parasitic powder particle debris still remains. Additionally, it does not seem like the large structures were affected at all by the polishing; the peaks of those particles are at a similar height to the peaks of the as-printed material. From here, the energy density remained the same, but the number of pulses was increased, as seen in Figure 89. 


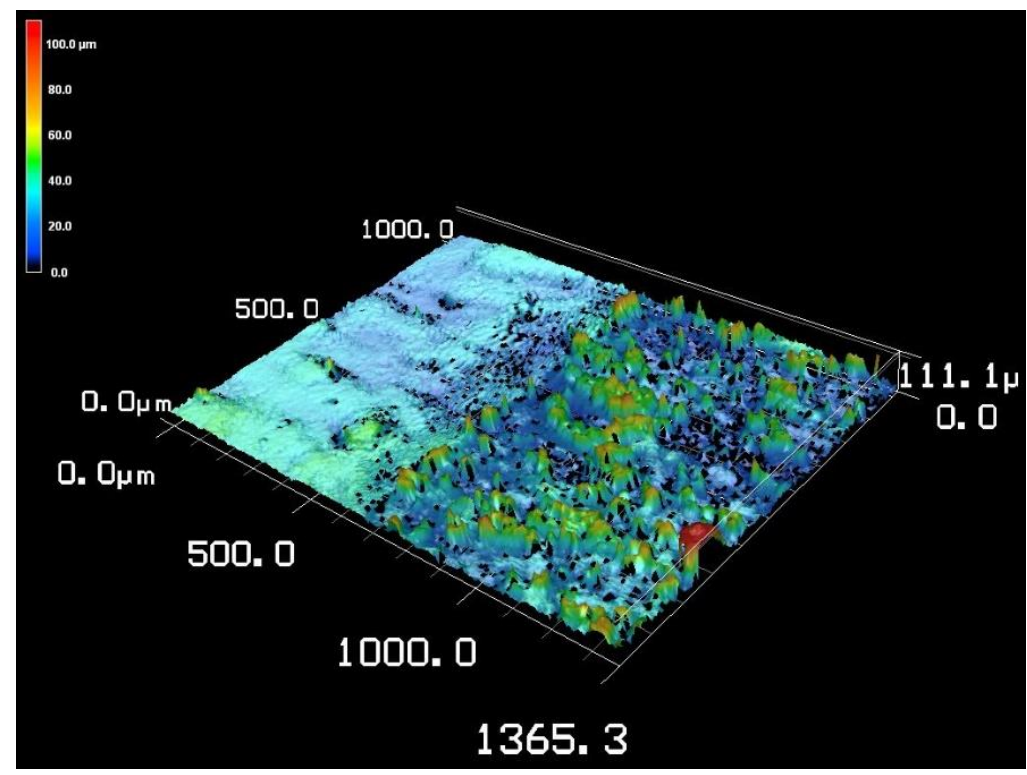

Figure 89: Laser profilometer measurement of area exposed to $4 \mathrm{~J} / \mathrm{cm}^{2}$ energy density and 125 pulses.

Again, this laser profilometer measurement image was taken on the border of the scanned area and the as-printed material. There is a large improvement for these scanning parameters, as there are no peaks at all in the polished area. The same characteristic ripples of the re-solidification process can be seen in the image. These contribute to what remains of the surface roughness for the part, as it is not a perfectly flat surface. Again, the number of pulses was increased while keeping the energy density constant, shown in Figure 90. 


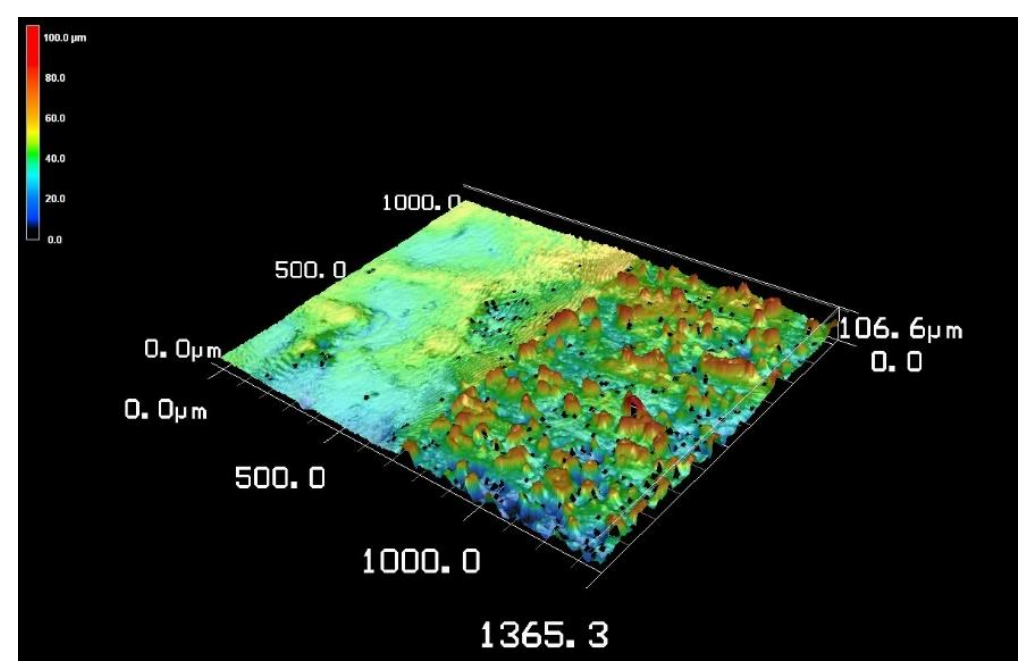

Figure 90: Laser profilometer measurement of area exposed to $4 \mathrm{~J} / \mathrm{cm}^{2}$ energy density and 175 pulses.

This was the highest energy density and number of pulses tested. When compared to the as-printed surface, it is clear that the polished surface is superior. Overall, the polished surface is the same color, indicating that it is has little to no roughness remaining. However, some previous structures still remain largely intact. This means that the energy density was still too low for full scale re-melting of the surface to occur. However, the measured surface roughness of these sample was $3.2 \mu \mathrm{m}$, which is a large improvement over the $8 \mu \mathrm{m}$ initial roughness. Aside from the laser profilometer measurements, measurements with the Mitutoyo profilometer were made as well. Each of the three striped scan areas were measured with and across the laser scan direction. An ANOVA model was produced for these measurements, shown in Figure 91. 


\section{Analysis of Variance}

\begin{tabular}{lrrrrr} 
Source & DF & Adj SS & Adj MS & F-Value & P-Value \\
\hline Scanned Area & 2 & 0.08710 & 0.04355 & 0.27 & 0.769 \\
Direction of Measurement & 1 & 2.19102 & 2.19102 & 13.51 & 0.003 \\
Scanned Area*Direction of Measurement & 2 & 1.23168 & 0.61584 & 3.80 & 0.053 \\
Error & 12 & 1.94580 & 0.16215 & & \\
Total & 17 & 5.45560 & & &
\end{tabular}

Figure 91: ANOVA model for the average measured surface roughness of the different scanned areas.

The interaction of the scanned area and direction of measurement was not considered to be significant in this model, due to the large influence from the direction of measurement. Therefore, direction of measurement was found to significantly affect the measured surface roughness, while the scanned area did not significantly affect the measured surface roughness. The residual plots for the ANOVA model can be seen in Figure 92.

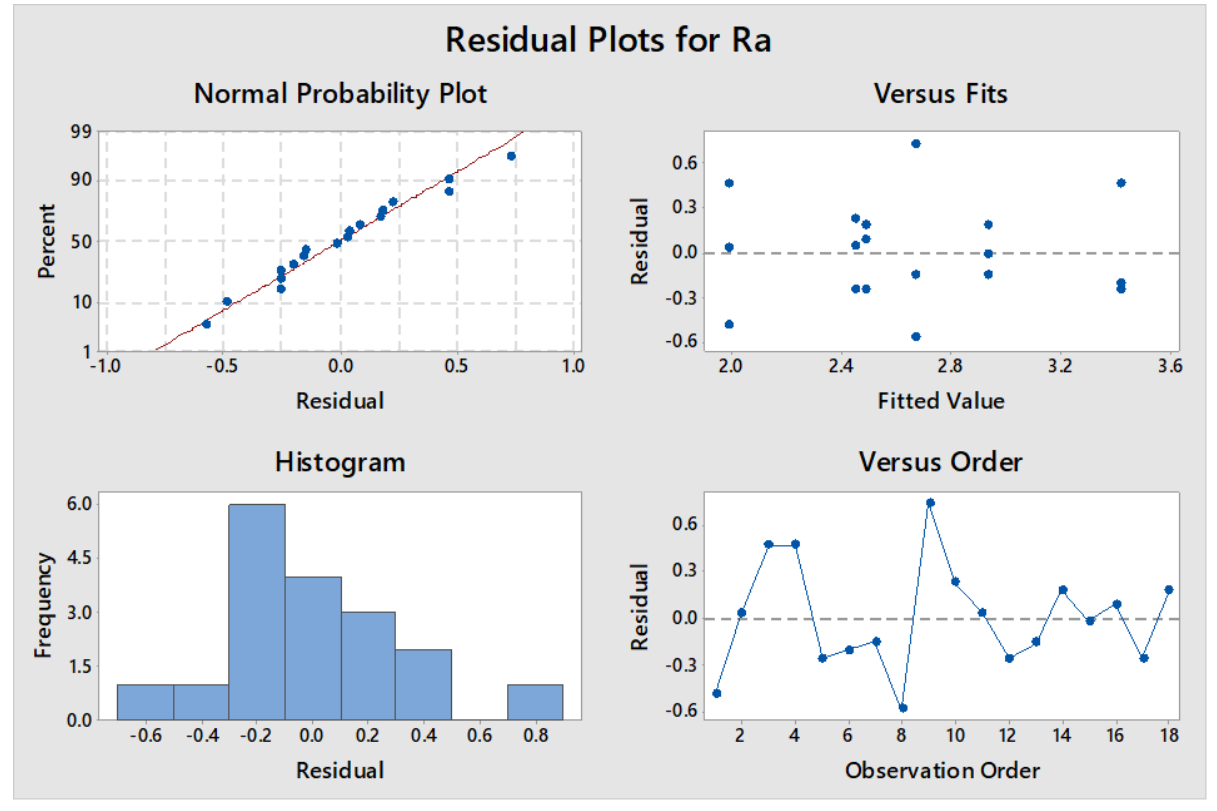

Figure 92: Four-in-one plot of residuals for the laser polishing average measured surface roughness models. 
There is nothing unusual about the residual plots of the average measured surface roughness. The data is normal, there is no significant difference in variance between the samples, and there is no dependence on measuring order. The difference in means was investigated in Figure 93.

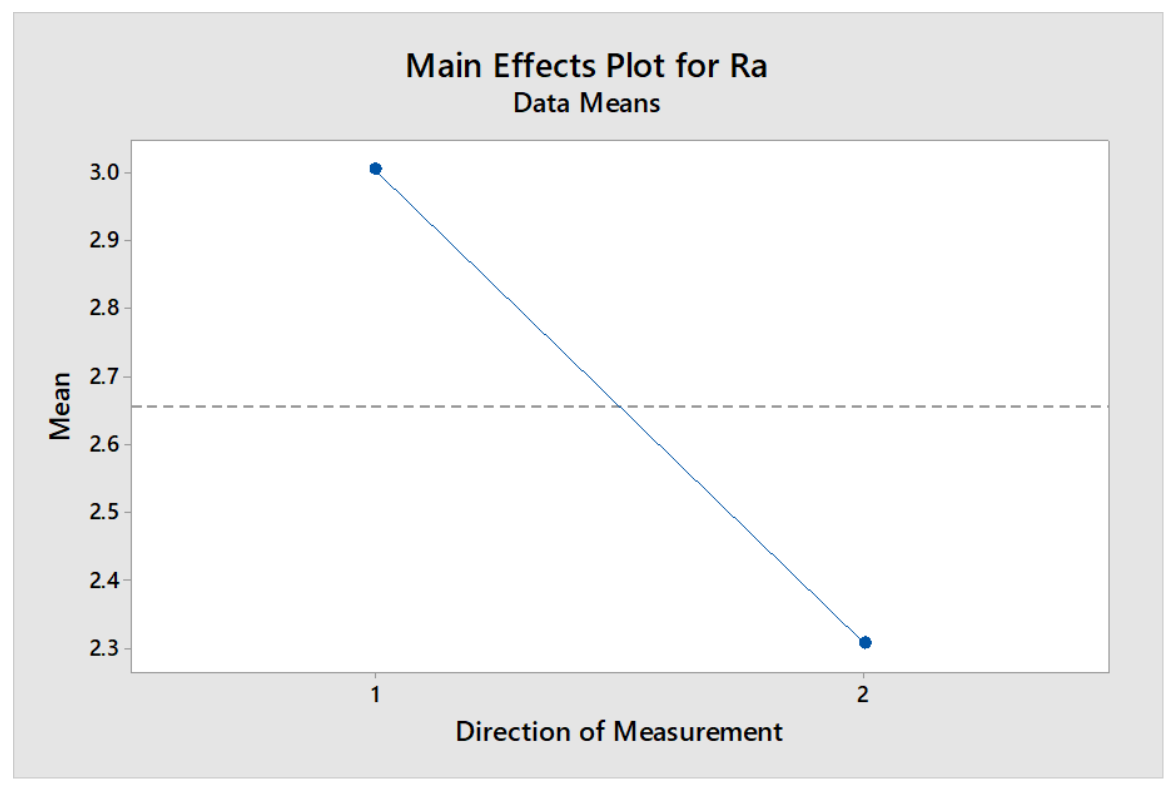

Figure 93: Main effects plot for measured surface roughness of laser polished surfaces; direction 1 was measured across the scan path, while direction 2 was measured with the scan path.

The difference in means is approximately $0.7 \mu \mathrm{m}$. This difference is most likely due to step structures produced by the laser scan paths. As the tip of the profilometer moves across the surface, the step structures produce large surface roughness readings. Overall, this means that the laser polishing does not produce an isotropic surface.

\subsection{Discussion}

Based on the results, the electropolishing happened best on the surfaces that were upward facing during printing and upward facing during electropolishing. There was a significant improvement in surface roughness during the electropolishing process, but only on the 
outside most surfaces, such as the face and the first few millimeters of the entrance of the holes. Looking at the SEM images however, it is clear that the feature size of the parasitic powder particles was too large for the electropolishing process to handle. In many cases, the surface of the parasitic powder particle and the area surrounding it was smoothed, but the structure remained largely intact. This led to the small drop in surface roughness seen. Additionally, these results are inconsistent with literature, which indicate the ability of the process to reduce the surface roughness to the order of hundreds of nanometers (Urlea \& Brailovski, 2017), while this process produced a surface, at best, that was about $3 \mu \mathrm{m}$ $R a$, even after a 40-minute bath time. It is possible that the experiments in the literature used different polishing parameters, or had smaller starting surface roughness, which led them to be able to achieve lower surface roughness. To further the point, while the downward facing surfaces had a larger absolute difference between their starting and finishing surface roughness, their final surface roughness was higher than the starting surface roughness for the upward facing surfaces. Therefore, it appears that electropolishing reaches a practical limit to the amount of material removal that can occur before it reaches a material removal rate that is not useful.

Additionally, the hole type was significant in determining how much material was removed. This is most likely due to a mixture of different electrolyte flow through the various channels, as well as more surface area being exposed to the cathode to promote dissolution. This led to more material being removed at certain corners as well. Most likely, these corners had a higher removal rate than the surrounding areas, causing a small channel to form, leading to increased material removal, leading to rounded corners. Furthermore, there was significance in the measurements of edge to edge across all of the 
holes. This shows that while the corners were experiencing varying rates of ion dissolution, the flats experienced mostly the same. However, this does not match the findings of the surface roughness data, which showed that edge two experienced significantly more polishing than edge one.

When considering the penetration depth of the electropolishing process, there are definite problems with decreasing feature size and increasing complexity. It is clear to see that as the diameter of the small holes decreased, the depth of polishing was smaller.

Additionally, the diamond through holes that had more surface area directly exposed to the cathode in the tank were, on average, polished more. There exists electropolishing probes that can be used to increase the potential of these surfaces in order to decrease this problem; however, they were not used in this particular study. To safely use these probes, the feature size has to be large enough to fit them, meaning small internal channels will likely never be successfully electropolished, unless a large amount of material is removed.

In this study, two laser polishing experiments were performed: first, the optimization of laser parameters for polishing 316L. At the optimal parameters, the surface roughness was reduced from $8 \mu \mathrm{m}$ to $2.3 \mu \mathrm{m}$. However, this does not match literature, which claimed that a sub-micron surface finish was achievable. This is due to the polishing company performing micro polishing without first performing macro polishing. As noted in the literature, micro polishing can only produce a sub-micron surface finish if it starts with a relatively smooth surface to begin with (Willenborg, 2011). Therefore, to treat a surface as rough as an additively manufactured one, a polishing process prior to micro polishing must be performed. This could be a macro laser polishing, or even 
electropolishing. As seen in many of the images of the polished surface, the large particle deposits were unaffected by the laser and did not melt, except at the surface. There was a difference in the size of particles the laser could deal with when comparing the different energy densities tested; the $4 \mathrm{~J} / \mathrm{cm}^{2}$ energy density was able to deal with more particles than the $1 \mathrm{~J} / \mathrm{cm}^{2}$ density. If the trend were to continue, it would make sense that a higher energy density would cause more melting at the surface and be able to handle larger particle sizes. However, it is possible that the higher energy density would result in more molten material in general, which could possibly re-solidify into a rougher surface than the lower energy densities tested in this study.

Additionally, there was no significant change in microstructure seen in the underlying metal as a result of the laser polishing. There was also no visible heat affected zone underneath the laser polished surface. The lack of microstructural evidence is most likely related to the low energy density applied by the laser. As can be seen in the laser profilometer images, the only affected material was the peak of the asperities. The molten material was re-distributed among the valleys to form the new surface, but the volume of re-allocated molten material did not carry enough thermal energy to affect the underlying material. Other possible explanations for the lack of microstructural changes and heat affected zone exist, such as equivalent cooling rates. The volume of molten material was so small, and had the bulk material to conduct heat into, meaning that it is possible that the cooling rate of the re-allocated material could have been similar to cooling rate experienced during the printing process, producing the same microstructure. However, the lack of new melt pool boundaries and continued existence of old melt pool boundaries 
from the printing process, suggests that this is not the case. The melt pool boundaries seen in Figure 81 are perpendicular to what they would be if new melt pools had formed.

The second experiment performed in this study was optimizing the scanning path of the laser. However, instead of the laser moving with objective lenses, the workpiece was moved by step motors. There was no discernable difference between the different scan patterns. All three shared the common traits of having step structures and ripples that resolidified approximately perpendicular to the scanning direction. These step structures are a result of the step motors having incremental steps, rather than a smooth scan path.

Finally, the surface produced was blackened due to oxidation. The laser scanning occurred without any shielding gas or vacuum, which allowed the material to oxidize. This may have led to some of the cracking seen in the surfaces, but it would not have affected the solidification structures too greatly. Additionally, the surface produced was still smooth, which makes it seem as though the oxidation did not affect the final surface roughness as much either. These oxides were not seen at any relevant depth into the microstructure.

\subsection{Conclusions}

- Electropolishing is a line-of-sight process; little to no material removal occurs in places where a strong potential cannot be applied.

- Electropolishing is not suited to be a start-to-finish polishing process; even after 40 minutes of polishing, the outer surface only improved by $5 \mu \mathrm{m}$.

- Electropolishing was strongly influenced by the shape of the passages, which changed how much surface area was directly exposed to the cathode. 
- Laser polishing was able to reduce the surface roughness of the $90^{\circ}$ surface from $8 \mu \mathrm{m}$ to $3.2 \mu \mathrm{m}$ with an energy density of $4 \mathrm{~J} / \mathrm{cm}^{2}$ and 175 pulses.

- The energy densities used in this study were not high enough to affect large surface structures.

- There was no difference in the roughness of the different surfaces produced by the different scanning patterns, but all three surfaces were anisotropic, with lower surface roughness in the direction of scan.

\section{Thin Disk Coin Design}

After observing the results of the initial study, it was decided to move forward with testing electropolishing. While the results of the interior polishing of the hexagon were not promising, a small amount of material was removed. Therefore, in an application where over-removal of material would cause the structure to fail, the small amount of material removed purely by electrolyte flow was investigated. To provide more relevance to the evaluation, the design chosen was a thin disk filled with lattice. The goal of this portion of the study was to remove just the parasitic powder particles from the surface of the lattice, which were plaguing the weight requirements of a similar Lawrence Livermore produced part, without affecting the integrity of the structure. Therefore, the sample needed a lattice structure that would be mechanically tested.

\subsection{Sample Design}

To truly capture the relevance of electropolishing to selective laser melting, the lattice needed to be designed in a hard to reach area. Initially, a tensile bar design was 
considered, but was ultimately dropped due to the concern that an outer skin would interfere with the testing of the lattice. Instead, a sample that could be compression tested was designed. The sample needed an interior chamber, as well as two parallel skins to be used for the compression test. Based on these criteria, a thin walled coin design was produced, as seen in Figure 94.

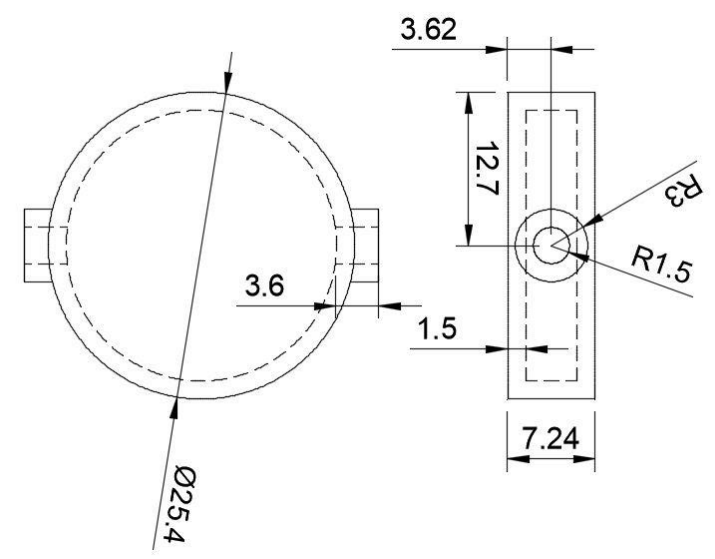

Figure 94: Dimensioned drawing of coin design. Dimensions in millimeters.

The diameter of the entry and exit holes was designed to inhibit the ability of the electropolishing fluid. To generate the lattice for the interior of the coin, nTopology Pro by Element was used. Cubic fluorite was used as the repeating structure; this is similar to the rhombic dodecahedron lattice structure. The key inputs and resulting outputs can be seen in Figure 95. 


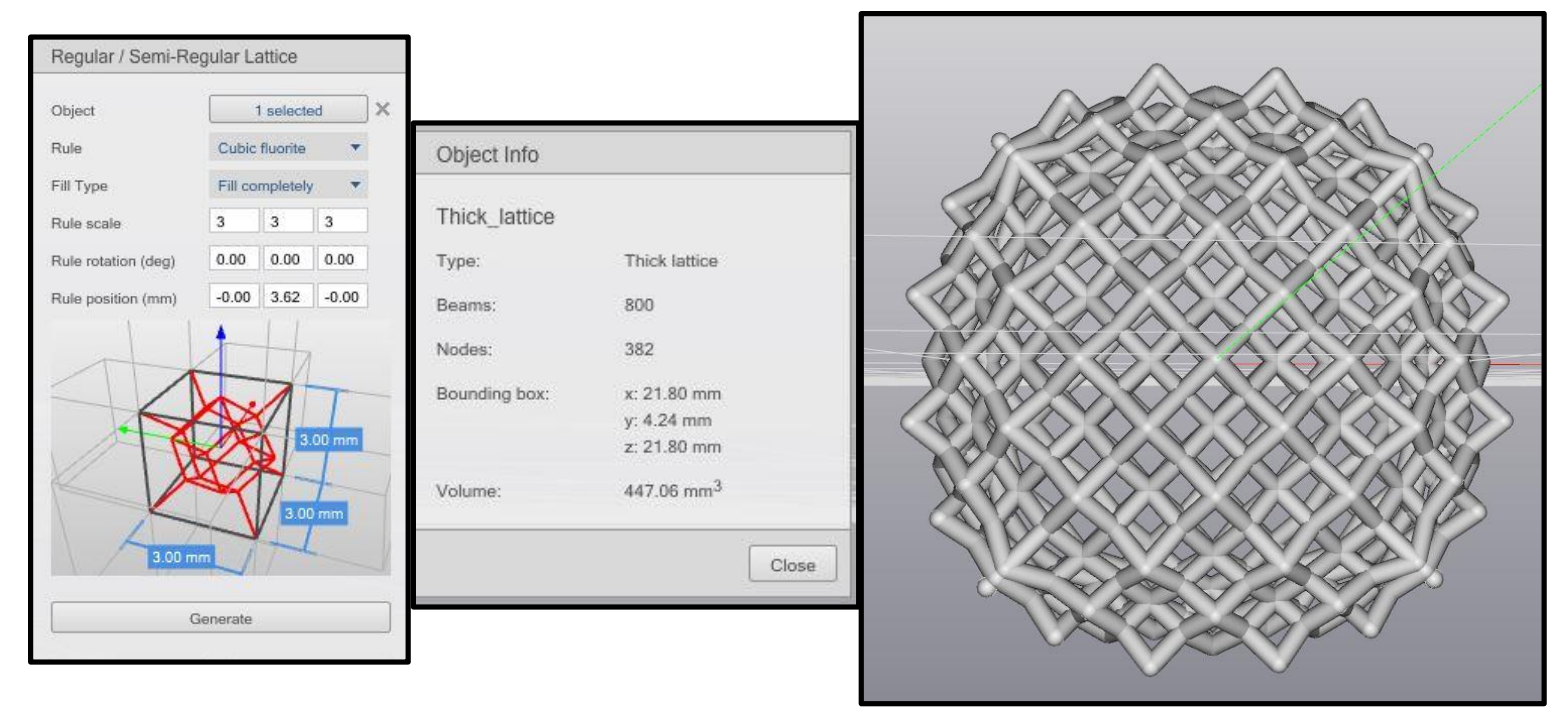

Figure 95: nTopology inputs and resulting lattice. The lattice unit cell is $3 \mathrm{~mm}^{3}$.

The lattice was designed to have $0.4 \mathrm{~mm}$ diameter struts. However, the opening to this coin was too small for the electropolishing probe to get near the lattice. Therefore, a secondary coin sample was designed with a larger opening to allow for the probe.

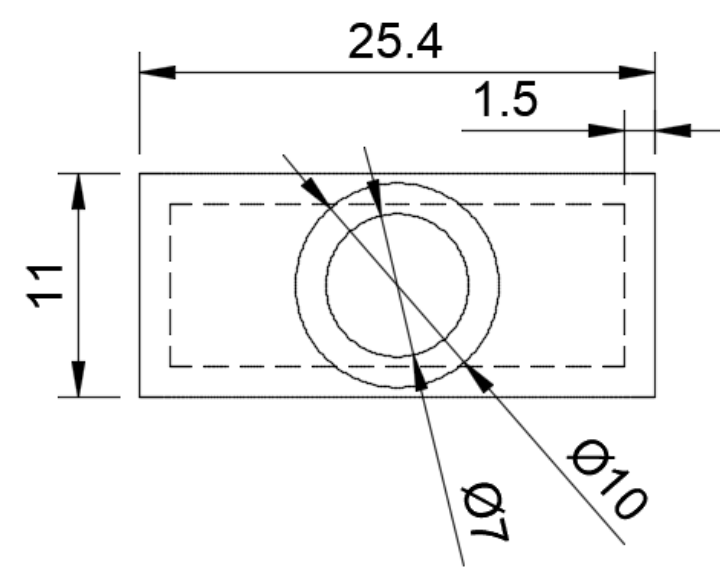

Figure 96: Dimensions of larger coin sample. All dimensions in millimeters.

However, the lattice interfered with the electropolishing probe, so the larger entrance diameter of this sample served to find the difference in the ability of the electrolyte to 
flow through the sample. The same diameter and lattice output was used for the larger coin sample as the smaller sample. Samples were printed as seen in Figure 97.

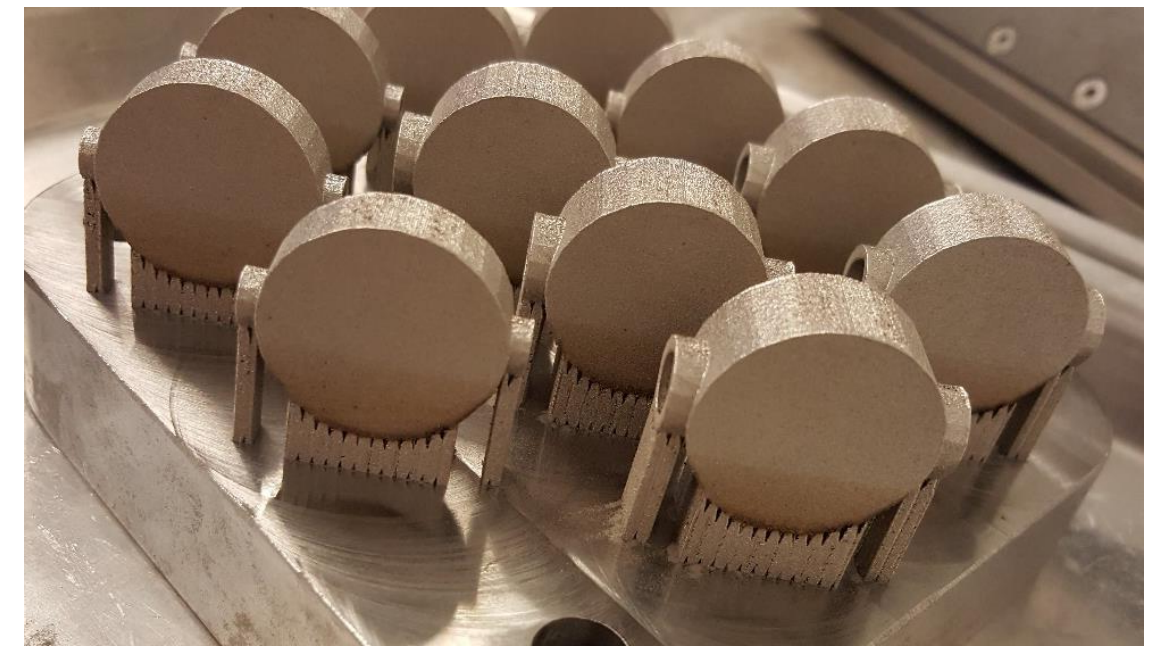

Figure 97: Coin samples still attached to build plate.

Twelve total samples were printed; 6 of the smaller design, and 6 of the large design. Samples were removed from the build plate using a bandsaw. Any remaining support structure was removed with a hammer and chisel. Once a sufficient amount of support structure was removed, samples were weighed using a precision balance, as seen in Figure 98.

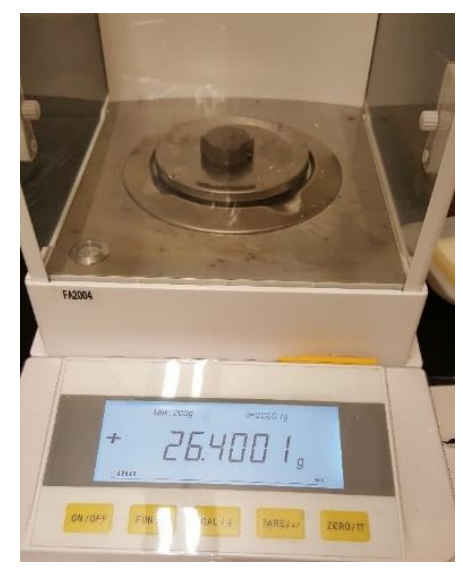

Figure 98: Large coin sample being weighed on the precision balance. 
Once the samples were weighed, they were sent to be electropolished. The exterior of the samples was masked to ensure that only the interior surface was polished. Three samples of both the small and big coin designs were electropolished. Table V summarizes the experimental design.

Table V: Experimental Design for Polishing of Thin Disk Coin Designs Sample Probe Used Time in Bath Bath Content Number

Polished

\begin{tabular}{l|llll} 
Small Coin & No & 5 minutes & Phosphoric and 3 \\
& & & Sulfuric \\
Large Coin & No & 5 minutes & Phosphoric and 3 \\
& & & Sulfuric
\end{tabular}

The wall thickness of the coin disk design prevented compression testing from providing any data relevant to the strength of the lattice. Therefore, 0.5 " $\mathrm{x} 0.5$ " squares were cut out of the center thickness of the disks using a waterjet. The waterjet was used because the high pressure cutting action would not damage the lattice the same way a cut-off saw might. Figure 99 shows the samples after removal from the thin disks.
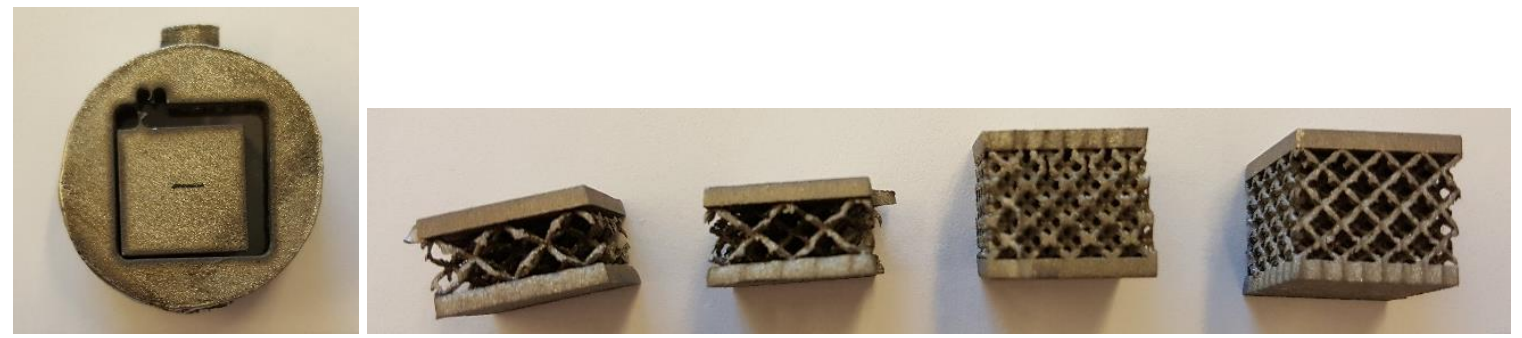

Figure 99: (Left) Small coin lattice immediately following extraction with a waterjet. (Right) The resulting lattices after being removed from their respective disks. 
While the waterjet was fairly accurate, there was some difficulty removing lattice at the exact same spot each time, resulting in a slightly different appearance of the lattices on the outer-most edge of the cubes. Due to the small size of the disks, the samples were not able to be clamped and were instead press-fit into a cut out in a plywood board, which caused the previously mentioned variations. However, this was determined not to affect the results of the compression test significantly by the resulting compression tests. Figure 100 shows the larger coin lattice after removal from the coin.
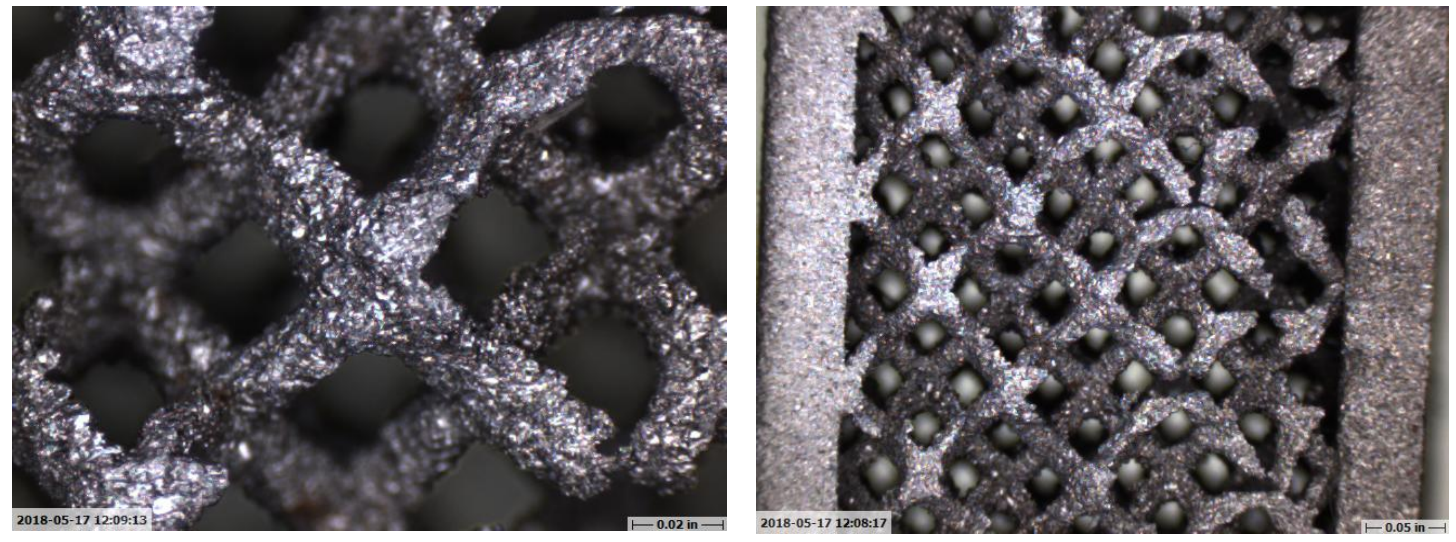

Figure 100: Optical images of side view of larger lattice; significantly rough surface is clearly visible.

The surfaces of the lattice struts are covered in parasitic powder particles. However, it is clear that the waterjet cut did not significantly affect the lattice, at least visually, as the struts on the inside of the rectangular prism are still intact.

Prior to compression testing, samples were hardness tested using the HRB scale. The samples were hardness tested on the thickness of the wall, due to the hardness tester providing enough force to deform the smaller lattices plastically. The results of the hardness tests are summarized in Table VI. 
Table VI: Hardness Measurements of Thin Disk Samples

\begin{tabular}{|c|c|c|c|c|}
\hline Sample & $\begin{array}{c}\text { Measurement 1 } \\
(\mathrm{HRB})\end{array}$ & $\begin{array}{c}\text { Measurement 2 } \\
(\mathrm{HRB})\end{array}$ & $\begin{array}{c}\text { Measurement 3 } \\
(\mathrm{HRB})\end{array}$ & $\begin{array}{c}\text { Average } \\
(\mathrm{HRB})\end{array}$ \\
\hline Large disk & 102.1 & 103.8 & 106.6 & 104.2 \\
\hline Small disk & 102.9 & 102.8 & 105.7 & 103.8 \\
\hline
\end{tabular}

These hardness values are similar to those reported in literature for non-heat treated 316L. These samples were not heat treated due to a faulty vacuum furnace and time constraints.

\subsection{Compression Testing and Results}

Three control samples of the small coin design and two of the large coin design were compression tested on a Shimadzu AG-X universal testing machine. The strain rate used for the tests was $1 \mathrm{~mm} / \mathrm{min}$. A small lattice can be seen in Figure 101.

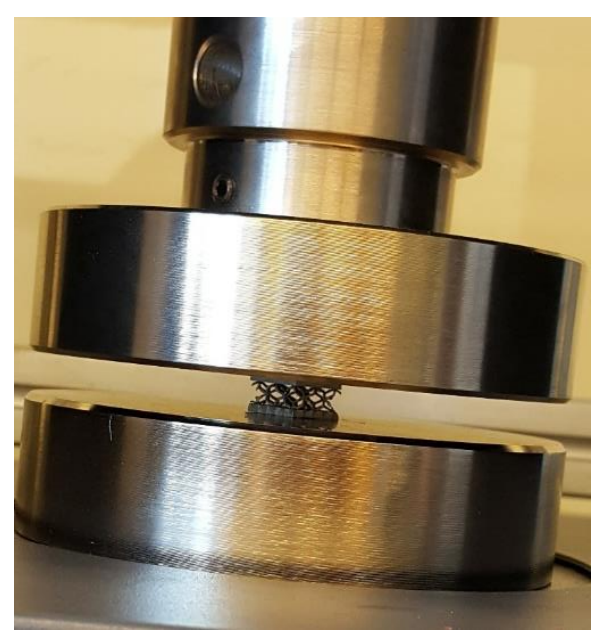

Figure 101: Small lattice after removal from disk, in position to be compression tested. 
The output of the machine was load in Newtons and displacement in millimeters. This was converted to stress and strain using the cross-section and height, respectively, of the samples being tested. The samples were tested until it was clear that the sample had entered the densification region of the compression curve, where the compression test is similar to a bulk material. Figure 102 shows the results for the large lattice specimens.

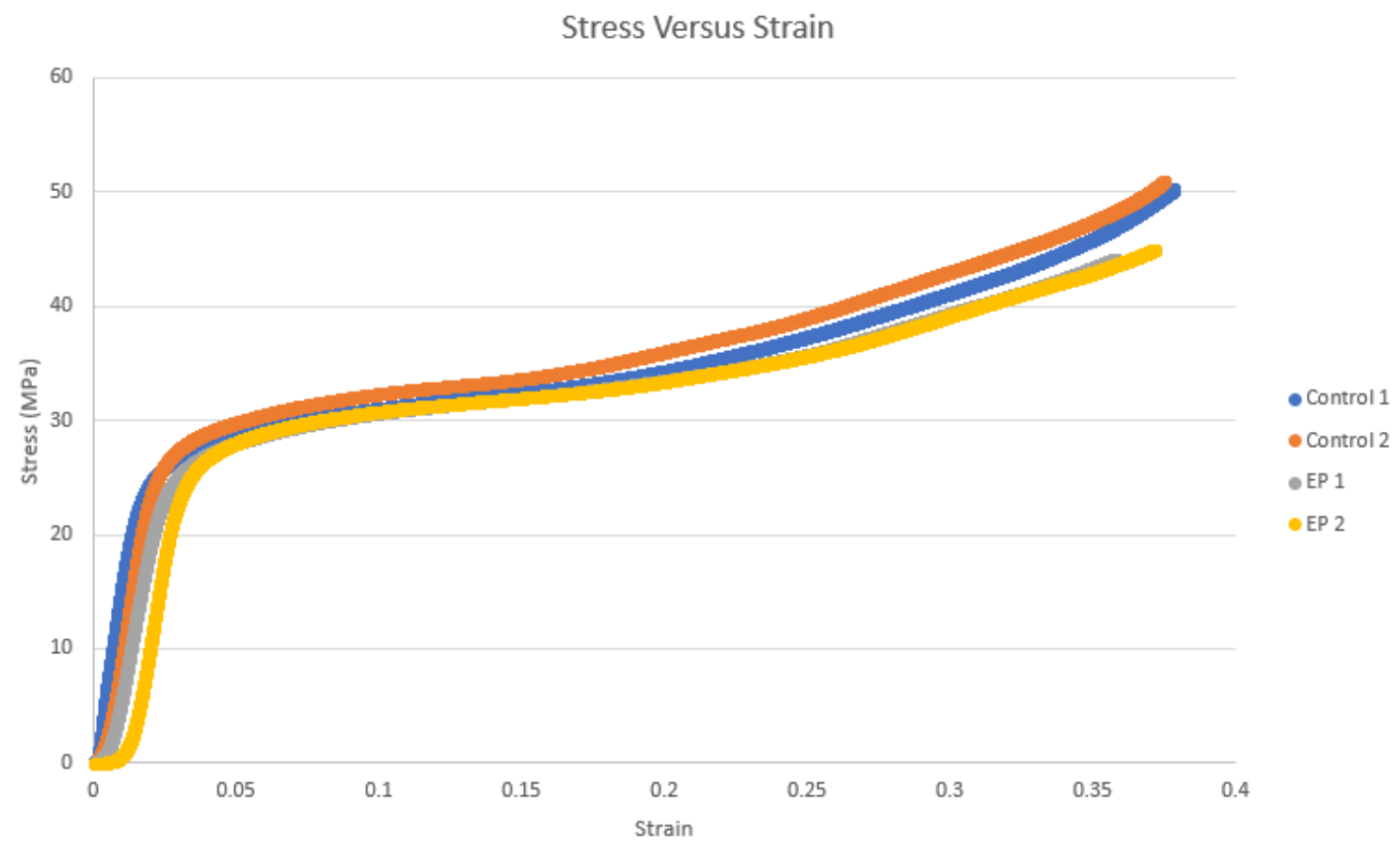

Figure 102: Stress versus strain curve for large lattice samples.

The compressive data of the four samples tested were largely the same, having similar densification strains and initial collapse stresses. This means that although the precision of the water jet cuts was not high, the bulk lattice contained in the sample was primarily responsible for the mechanical response, and not the lattice on the outermost edge of the sample. Additionally, there was no significant difference in the mechanical behavior of the as-printed lattice and the electropolished lattice, meaning that the small amount of polishing that occurred did not affect the integrity of the structure. The stress versus 
strain curve produced for these specimens is typical of a bending-dominated lattice, clearly displaying the four different regions. After the large specimens were tested, the small lattice samples were tested. The resulting stress-strain curve can be seen in Figure 103.

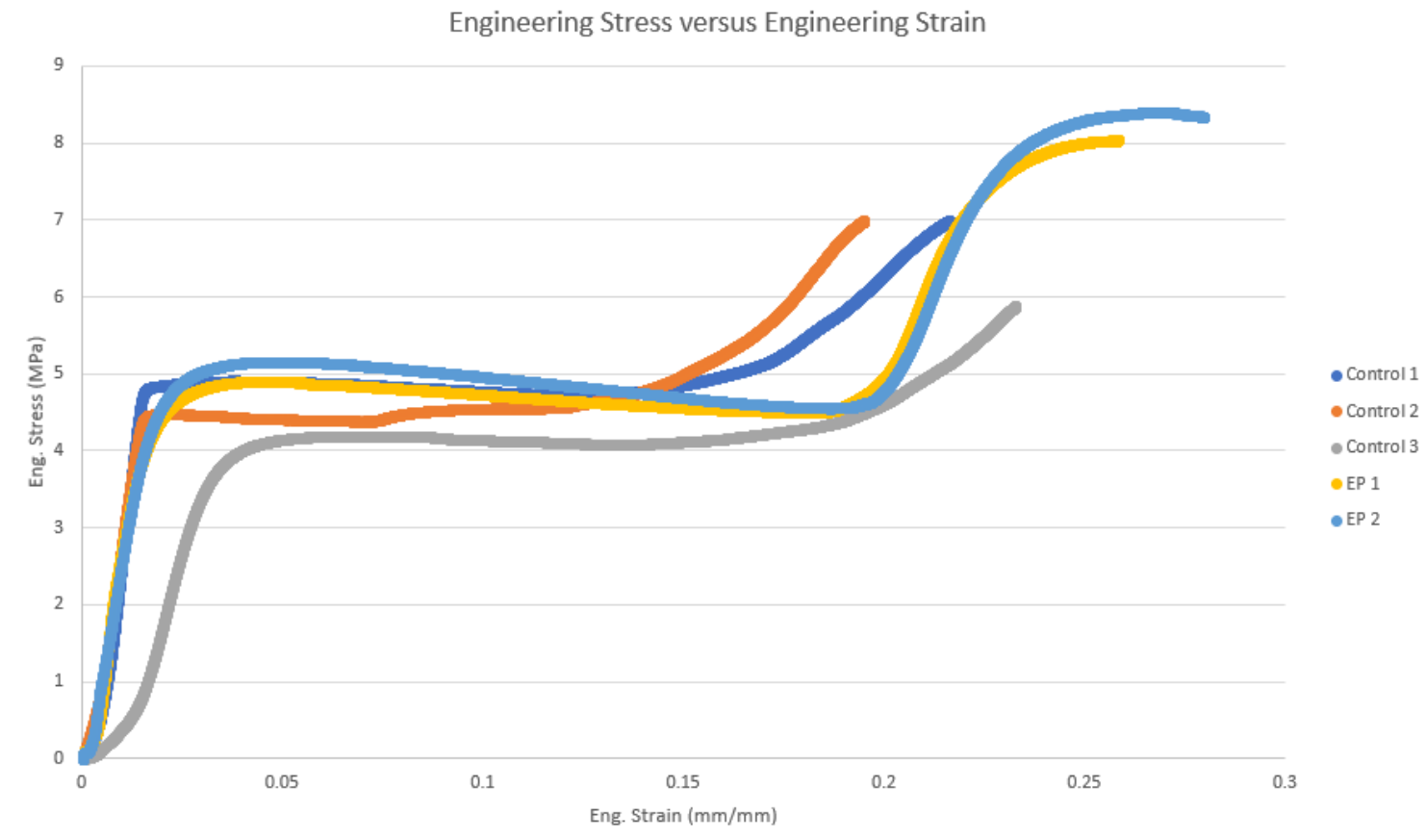

Figure 103: Stress versus strain for small lattice samples.

The small lattice samples displayed slightly more variation than the large lattice control samples. Additionally, the mechanical strength of these lattice samples was greatly diminished. Both the variation and mechanical strength difference can be explained by the difference in cell size between the lattices. Due to the large cell size of the lattice, the large bulk of lattice structure contained within the large lattice did not exist in the small lattice, meaning the precision of the water jet cuts mattered much more. The difference in mechanical strength is also known to depend strongly on the cell size of the lattice structure (Recep Gümrük et al., 2013). Again, however, there was no significant 
difference in the strengths of the unprocessed and processed lattices. The summarized testing data can be seen in Table VII.

Table VII: Summarized Compression Test Data for Thin Disk Lattice Samples

\begin{tabular}{|c|c|c|c|c|}
\hline Sample & $\begin{array}{c}\text { Sample } \\
\text { Dimensions } \\
(\mathbf{m m})\end{array}$ & $\begin{array}{c}\text { Compressive } \\
\text { Modulus } \\
\text { (MPa) }\end{array}$ & $\begin{array}{c}\text { Initial } \\
\text { Collapse } \\
\text { Stress (MPa) }\end{array}$ & $\begin{array}{c}\text { Densification } \\
\text { strain }\end{array}$ \\
\hline \multirow[t]{2}{*}{$\begin{array}{c}\text { Large Thin Disk } \\
\text { Control }\end{array}$} & $\begin{array}{c}12.41 \times 12.36 \\
\times 10.97\end{array}$ & 1148.3 & 22.6 & 0.3 \\
\hline & $\begin{array}{c}12.38 \times 12.41 \\
\times 11.06\end{array}$ & 1632.6 & 23 & 0.3 \\
\hline \multirow[t]{3}{*}{$\begin{array}{c}\text { Small Thin Disk } \\
\text { Control }\end{array}$} & $\begin{array}{c}12.29 \times 12.34 \\
\times 7.24\end{array}$ & 463.1 & 4.7 & 0.17 \\
\hline & $\begin{array}{c}12.25 \times 12.39 \\
\times 7.20\end{array}$ & 374.3 & 4.3 & 0.15 \\
\hline & $\begin{array}{c}12.34 \times 12.30 \\
\times 7.21\end{array}$ & 202 & 3.65 & 0.2 \\
\hline \multirow[t]{2}{*}{$\begin{array}{l}\text { Large Thin Disk } \\
\text { Electropolished }\end{array}$} & $\begin{array}{c}12.31 \times 12.34 \\
\text { x } 10.97\end{array}$ & 1344.7 & 25 & 0.25 \\
\hline & $\begin{array}{c}12.27 \times 12.32 \\
\times 10.97\end{array}$ & 1054.6 & 26.5 & 0.25 \\
\hline \multirow[t]{2}{*}{$\begin{array}{l}\text { Small Thin Disk } \\
\text { Electropolished }\end{array}$} & $\begin{array}{c}12.36 \times 12.39 \\
\times 7.3\end{array}$ & 339.5 & 4.6 & 0.2 \\
\hline & $\begin{array}{c}12.29 \times 12.35 \\
\times 7.22\end{array}$ & 304 & 4.6 & 0.2 \\
\hline
\end{tabular}

The compressive modulus of all the specimens varied significantly, but there was little variance in the densification strains and initial collapse stresses. Additionally, the small amount of variance seen can also be explained by printing orientation. The control samples used were printed rotated $45^{\circ}$ compared to the processed samples. Therefore, there is no variance in the samples due to processing. 
To measure the amount of material removed, samples were weighed before and after electropolishing. The results are summarized in Table VIII.

Table VIII: Mass Loss Measurements for Electropolished Thin Disk Lattice Samples

$\begin{array}{llllll}\text { Sample } & \text { As-Printed } & \text { Post- } & \text { Change in } & \text { Average } & \text { Average } \\ \text { Weight } & \text { Electropolishing } & \text { Weight } & \text { Change in } & \text { Change in } \\ \text { (grams) } & \text { Weight (grams) } & \text { (grams) } & \text { Weight } & \text { Weight } \\ & & & \text { (grams) } & (\%)\end{array}$

\begin{tabular}{|r|c|c|c|c|c|} 
Large Thin & 26.168 & 25.806 & -0.362 & $-0.286 \pm 0.066$ & $-1.08 \pm$ \\
\cline { 2 - 4 } Disk & 26.304 & 26.05 & -0.254 & & 0.25 \\
\cline { 2 - 4 } & 26.361 & 26.119 & -0.242 & & \\
\cline { 2 - 4 } Small Thin & 17.142 & 16.988 & -0.154 & $-0.155 \pm 0.021$ & $-0.902 \pm$ \\
\cline { 2 - 4 } Disk & 17.193 & 17.016 & -0.177 & & 0.125 \\
\cline { 2 - 4 } & 17.194 & 17.06 & -0.134 & & \\
\hline
\end{tabular}

When considering pure mass removal, the larger sample lost more mass than the smaller sample, when experiencing the same treatment. However, when considering the percentage change in mass loss, there is no significant difference in the mass loss between the two samples, with both samples losing approximately $1 \%$ of their mass. Scanning electron microscopy of the sectioned samples was employed to investigate where on the samples this material removal occurred.

\subsection{SEM Images}

SEM images were taken of samples before and after compression testing to characterize the surface condition of the struts. The large lattice control specimen was imaged first, shown in Figure 104. 


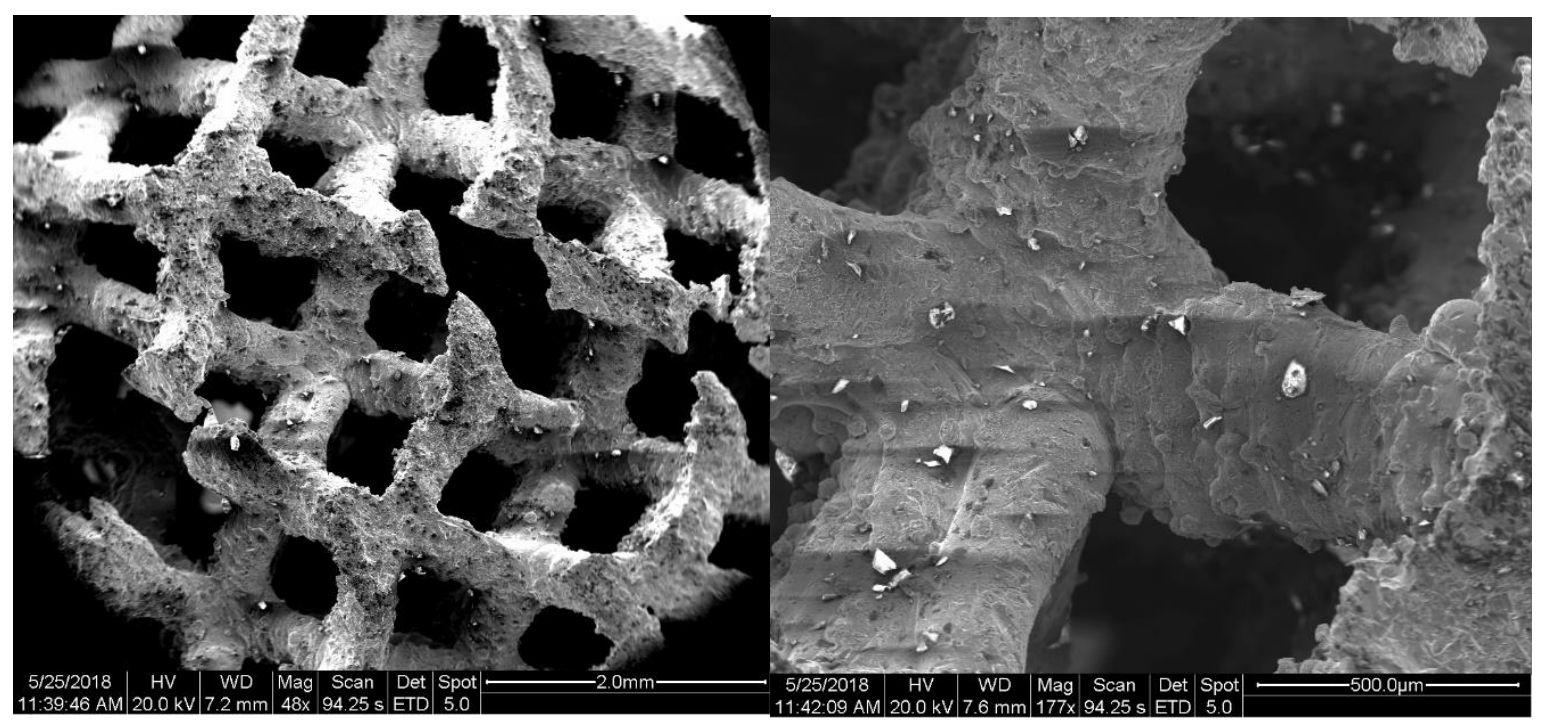

Figure 104: SEM images of large lattice sample prior to compression testing, demonstrating the poor surface condition of as-printed lattice. Strut diameter $=0.4 \mathrm{~mm}$.

The surface of the struts is covered in small parasitic particles; additionally, the staircase structure can clearly be seen. Importantly, it can be seen that some areas of the lattice have had their nodes removed, preventing the struts from interacting from each other, and the lattice from holding a load in that area. Effectively, those struts have been removed from usefulness for a mechanical test. However, it is assumed that the lack of presence of these nodes did not significantly affect the results of the mechanical stress, as the bulk of the lattice was still intact and able to carry and transfer the mechanical load. The large lattice sample was also imaged after compression testing, shown in Figure 105. 


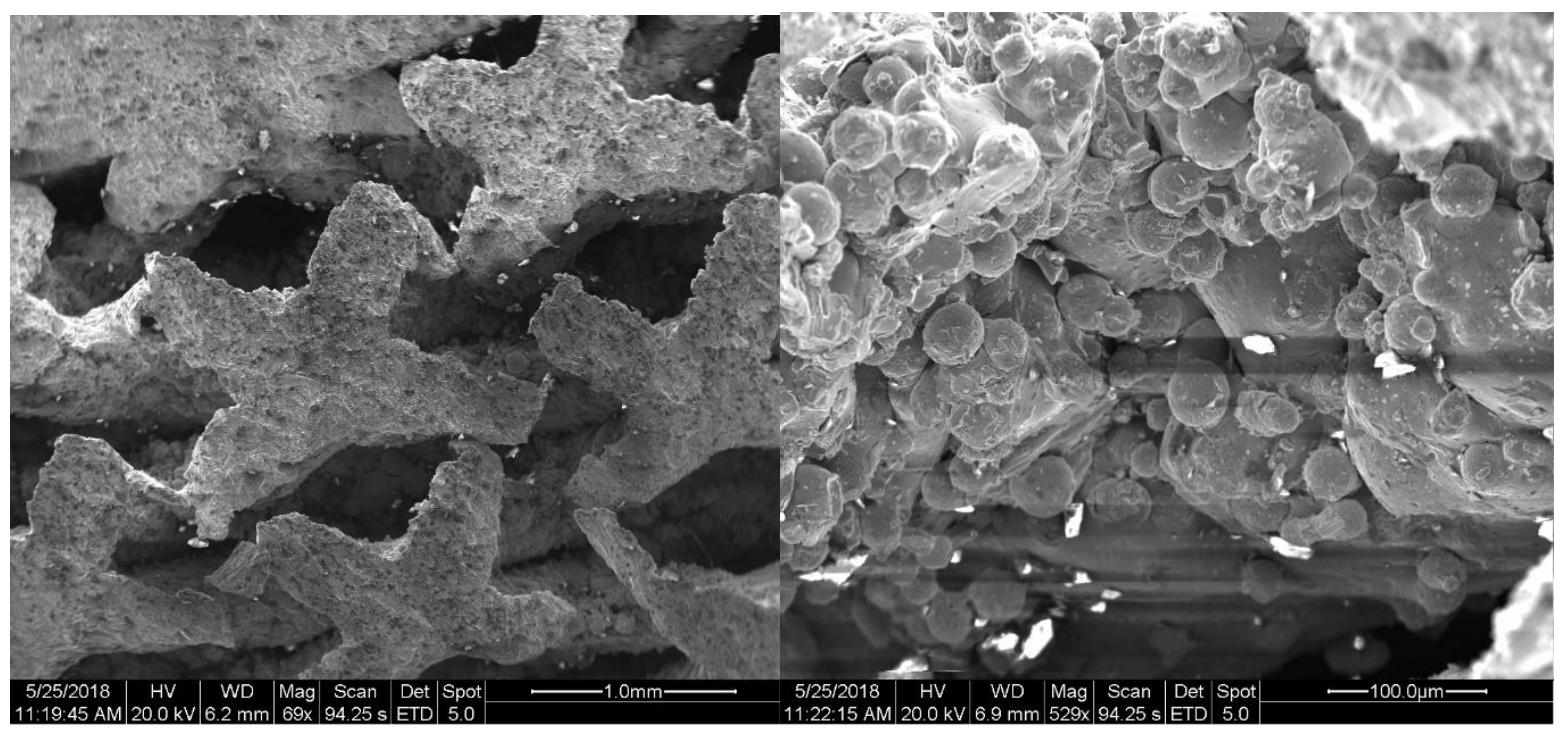

Figure 105: SEM images of the large lattice sample post compression test. (Left) 69x, showing failed struts. (Right) 529x, showing gathering of parasitic particles on failed corner.

As expected, the compressive load caused the structure to plastically deform and become much closer. The surface of the failed struts had many more powder particles visible on the surface than before the compression test. These particles did not appear to have plastically deformed, due to maintaining their spherical shape. The higher concentration of particles is a good indicator of how much plastic deformation occurred on the struts, showing that they were able to move on the surface as the struts deformed. SEM imaging of the failed small lattice sample shows similar results, shown in Figure 106. 


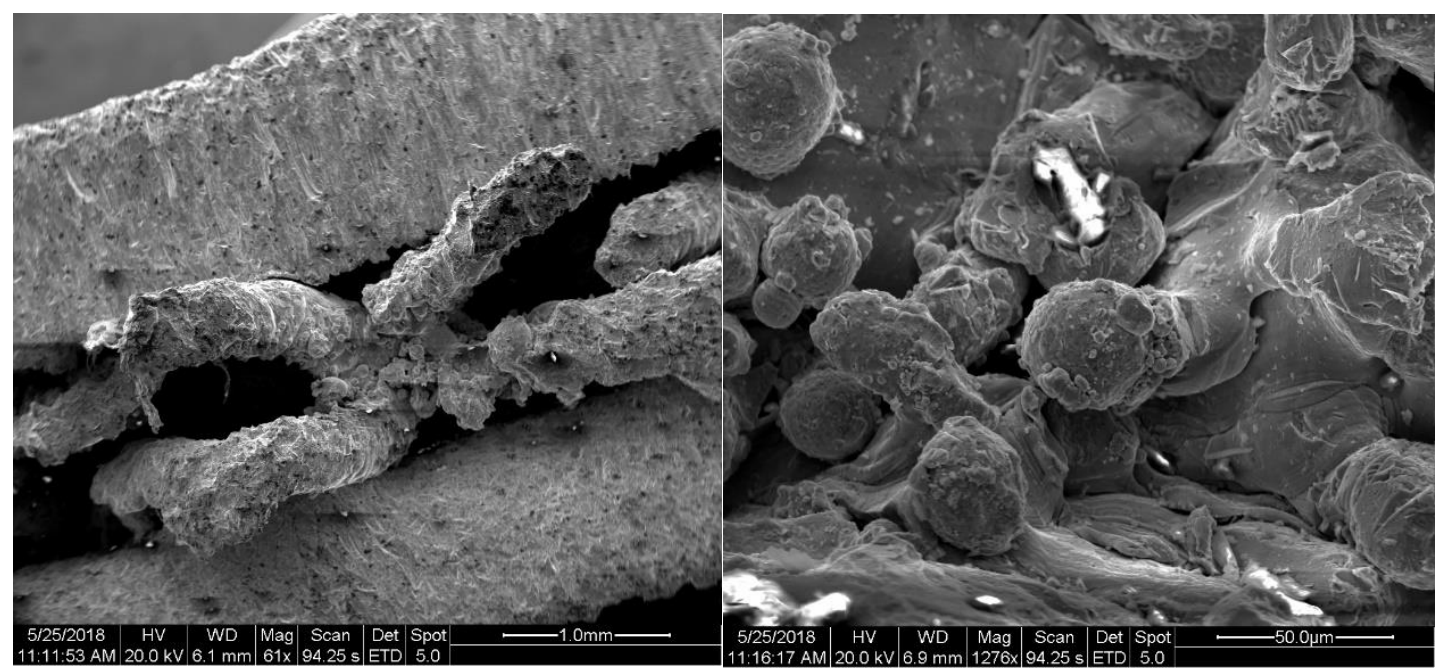

Figure 106: SEM images of failed small thin disk lattice structure. (Left) 61x, roughness of lattice is seen. (Right) 1276x, showing powder particles at the node a cell.

Similar to the large lattice, the compressive forces caused the small lattice to expand outward. Again, areas of high concentration of parasitic particles can be found on the surface of the failed struts. Following these tests, the electropolished samples were sectioned and imaged, shown in Figure 107.
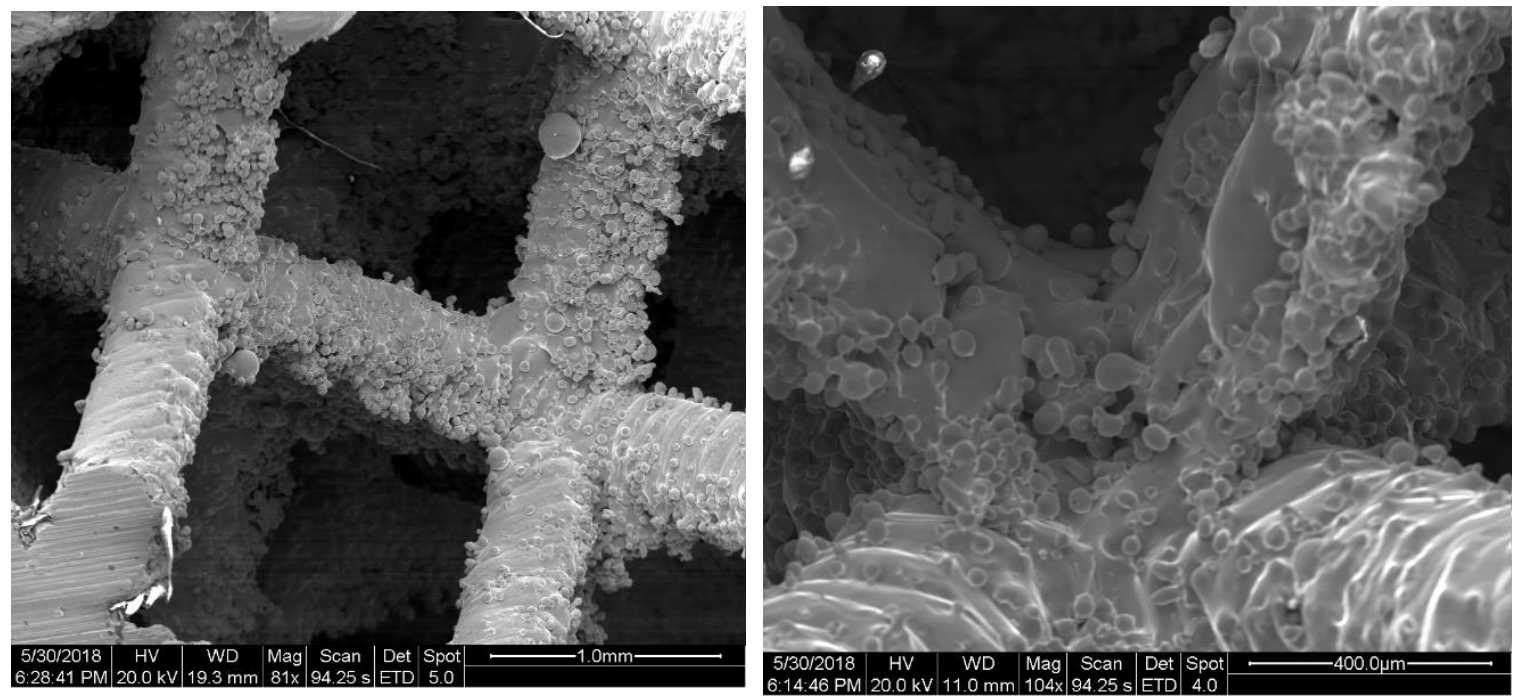

Figure 107: SEM images post electropolishing of (Left) small thin disk sample (Right) Large thin disk, both showing no polishing. 
As can be seen in both images, there was no significant polishing on either lattice sample, confirming that all weight loss likely happened at the entrance to the lattice parts. The size of entrance holes prevented electrolyte from easily flowing through the material, and when combined with the strong line-of-sight nature of electropolishing, prevented any polishing from occurring in the bulk of the lattice. As a side study, some unconstrained lattice samples were electropolished as well, shown in Figure 108.
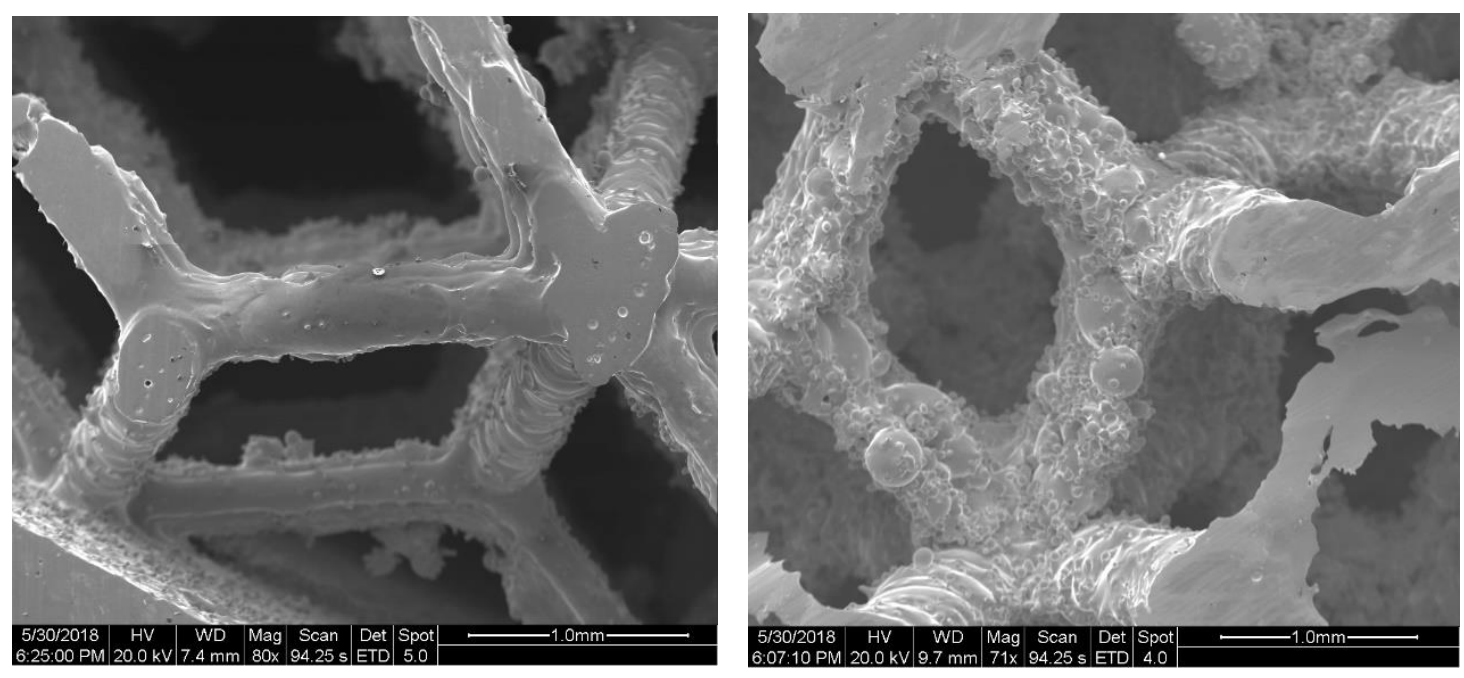

Figure 108: Unconstrained lattice samples showing some electropolishing, but still having issues with a good polish.

The left image shows the small sample, with some significant polishing happening directly at the surface, but it can clearly be seen that the area behind the front-most struts did not receive any polishing at all. The right image shows the large sample, processed under the same unconstrained conditions, showing no significant polishing at all. Therefore, even when not constrained by small entrances, electropolishing is not a good candidate for lattice structures. 


\subsection{Discussion}

The control lattice samples behaved as expected during the compression test, having clearly defined regions that are characteristic of a bending dominated structure. Interestingly, the compressive modulus varied largely between samples, while the densification strain and initial collapse strength remained constant. Additionally, the larger lattice sample was much stronger than the smaller lattice sample. This was expected as well, because the cell size of the larger lattice sample was much smaller than the small thin disk sample. The large gathering of parasitic particles on the surfaces of the struts post-compression test are also known to cause a reduction in the densification strain (R. Gümrük \& Mines, 2013).

The amount of mass removed by the electropolishing process of both lattice samples was 1\%. Similar to the circular through holes of the electropolished hexagon, most of the material removal occurred at the entrance of the holes and not through the bulk lattice. Due to the lack of material removal in the bulk lattice, there was no change in the mechanical strength of the lattices when tested in compression. The main reasons for the lack of polishing occurring in the bulk lattice are the small size of the entrance and exit holes preventing strong electrolyte flow, as well as the struts causing line-of-sight issues for the other struts being polished. The line-of-sight issues caused by the struts was confirmed by a small study within the study, where unconstrained struts were electropolished as well. The front most surfaces of these struts were generally electropolished, but all of the struts behind them were not able to be electropolished at all. Therefore, electropolishing is not a good candidate for lattice structures. 


\subsection{Conclusions}

Electropolishing was performed on two different thin disk lattice fill samples, resulting in a $1 \%$ weight loss. No difference in compressive strength was measured between the control samples and the electropolished samples. The miniscule weight loss and lack of difference was due to electrolyte flow and line-of-sight issues preventing the polishing of the bulk lattice. Additionally, even when unconstrained by the small entrance holes, the electropolishing process was only able to polish the frontmost struts, leaving the other struts unpolished. Therefore, it is not recommended to use electropolishing to treat the surface roughness of selectively laser melted lattice parts.

\section{Future Work}

To further investigate these processes, there is more work that can be done.

- Investigate laser polishing at higher energy density levels. Literature reported trials up to $20 \mathrm{~J} / \mathrm{cm}^{2}$, while the laser polishing in this study only reached $4 \mathrm{~J} / \mathrm{cm}^{2}$.

- Investigate the effects of laser polishing on thin-walled structures.

- Further investigate the ability of electropolishing to reduce surface roughness by changing process variables, such as cathode array, tank temperature, and potential. 


\section{Bibliography}

Attaran, M. (2017). The rise of 3-D printing: The advantages of additive manufacturing over traditional manufacturing. Business Horizons, 60(5), 677-688. https://doi.org/10.1016/j.bushor.2017.05.011

Bagehorn, S., Wehr, J., \& Maier, H. J. (2017). Application of mechanical surface finishing processes for roughness reduction and fatigue improvement of additively manufactured Ti-6Al-4V parts. International Journal of Fatigue, 102, 135-142. https://doi.org/10.1016/j.ijfatigue.2017.05.008

Baicheng, Z., Xiaohua, L., Jiaming, B., Junfeng, G., Pan, W., Chen-nan, S., ... Jun, W. (2017). Study of selective laser melting (SLM) Inconel 718 part surface improvement by electrochemical polishing. Materials and Design, 116, 531-537. https://doi.org/10.1016/j.matdes.2016.11.103

Bhaduri, D., Penchev, P., Batal, A., Dimov, S., Soo, S. L., Sten, S., ... Dong, H. (2017). Laser polishing of 3D printed mesoscale components. Applied Surface Science, 405, 29-46. https://doi.org/10.1016/j.apsusc.2017.01.211

Brøtan, V., Fergani, O., Sørby, K., \& Welo, T. (2016). Mechanical Properties of Biocompatible 316L Steel Rhombic Dodecahedron Lattice Structures, 2087-2094. Retrieved from https://sffsymposium.engr.utexas.edu/sites/default/files/2016/167Brotan.pdf

De Giorgi, C., Furlan, V., Demir, A. G., Tallarita, E., Candiani, G., \& Previtali, B. (2016). Laser Micro-polishing of Stainless Steel for Antibacterial Surface 
Applications. Procedia CIRP, 49, 88-93.

https://doi.org/10.1016/j.procir.2015.07.055

DebRoy, T., Wei, H. L., Zuback, J. S., Mukherjee, T., Elmer, J. W., Milewski, J. O., ... Zhang, W. (2018). Additive manufacturing of metallic components - Process, structure and properties. Progress in Materials Science, 92, 112-224. https://doi.org/10.1016/j.pmatsci.2017.10.001

Ding, J., Colegrove, P., Mehnen, J., Ganguly, S., Almeida, P. M. S., Wang, F., \& Williams, S. (2011). Thermo-mechanical analysis of Wire and Arc Additive Layer Manufacturing process on large multi-layer parts. Computational Materials Science, 50(12), 3315-3322. https://doi.org/10.1016/j.commatsci.2011.06.023

Farshidianfar, M. H., Khajepour, A., \& Gerlich, A. (2016). Real-time control of microstructure in laser additive manufacturing. The International Journal of Advanced Manufacturing Technology, 82(5-8), 1173-1186. https://doi.org/10.1007/s00170-015-7423-5

Fischer, P., Romano, V., Weber, H. P., Karapatis, N. P., Boillat, E., \& Glardon, R. (2003). Sintering of commercially pure titanium powder with a Nd:YAG laser source. Acta Materialia, 51(6), 1651-1662. https://doi.org/10.1016/S13596454(02)00567-0

Ghasri-Khouzani, M., Peng, H., Rogge, R., Attardo, R., Ostiguy, P., Neidig, J., ... Shankar, M. R. (2017). Experimental measurement of residual stress and distortion in additively manufactured stainless steel components with various dimensions. Materials Science and Engineering A, 707(July), 689-700. 
https://doi.org/10.1016/j.msea.2017.09.108

Gomez-Gallegos, A. A., Mill, F., \& Mount, A. R. (2016). Surface finish control by electrochemical polishing in stainless steel 316 pipes. Journal of Manufacturing Processes, 23, 83-89. https://doi.org/10.1016/j.jmapro.2016.05.010

Gu, D., \& Shen, Y. (2009). Balling phenomena in direct laser sintering of stainless steel powder: Metallurgical mechanisms and control methods. Materials and Design, 30(8), 2903-2910. https://doi.org/10.1016/j.matdes.2009.01.013

Gümrük, R., \& Mines, R. A. W. (2013). Compressive behaviour of stainless steel microlattice structures. International Journal of Mechanical Sciences, 68, 125-139. https://doi.org/10.1016/j.jimecsci.2013.01.006

Gümrük, R., Mines, R. A. W., \& Karadeniz, S. (2013). Static mechanical behaviours of stainless steel micro-lattice structures under different loading conditions. Materials Science and Engineering A, 586, 392-406. https://doi.org/10.1016/j.msea.2013.07.070

Gussone, J., Garces, G., Haubrich, J., Stark, A., Hagedorn, Y.-C., Schell, N., \& Requena, G. (2017). Microstructure stability of $\gamma$-TiAl produced by selective laser melting. Scripta Materialia, 130, 110-113. https://doi.org/10.1016/j.scriptamat.2016.11.028

Habibzadeh, S., Li, L., Shum-Tim, D., Davis, E. C., \& Omanovic, S. (2014). Electrochemical polishing as a 316L stainless steel surface treatment method: Towards the improvement of biocompatibility. Corrosion Science, 87, 89-100. https://doi.org/10.1016/j.corsci.2014.06.010 
Hebert, R. J. (2016). Viewpoint: metallurgical aspects of powder bed metal additive manufacturing. Journal of Materials Science, 51(3), 1165-1175. https://doi.org/10.1007/s10853-015-9479-X

Herzog, D., Seyda, V., Wycisk, E., \& Emmelmann, C. (2016). Additive manufacturing of metals. Acta Materialia, 117, 371-392. https://doi.org/10.1016/j.actamat.2016.07.019

Jung, J. H., Park, H. K., Lee, B. S., Choi, J., Seo, B., Kim, H. K., .. Kim, H. G. (2017). Study on surface shape control of pure Ti fabricated by electron beam melting using electrolytic polishing. Surface and Coatings Technology, 324, 106-110. https://doi.org/10.1016/j.surfcoat.2017.05.061

Khorasani, A. M., Gibson, I., Goldberg, M., \& Littlefair, G. (2016). A survey on mechanisms and critical parameters on solidification of selective laser melting during fabrication of Ti-6Al-4V prosthetic acetabular cup. Materials \& Design, 103, 348-355. https://doi.org/10.1016/j.matdes.2016.04.074

King, W. E., Barth, H. D., Castillo, V. M., Gallegos, G. F., Gibbs, J. W., Hahn, D. E., ... Rubenchik, A. M. (2014). Observation of keyhole-mode laser melting in laser powder-bed fusion additive manufacturing. Journal of Materials Processing Technology, 214(12), 2915-2925. https://doi.org/10.1016/j.jmatprotec.2014.06.005

Liu, Y., Zhang, J., \& Pang, Z. (2018). Numerical and experimental investigation into the subsequent thermal cycling during selective laser melting of multi-layer $316 \mathrm{~L}$ stainless steel. Optics and Laser Technology, 98, 23-32. https://doi.org/10.1016/j.optlastec.2017.07.034 
Ma, C., Andani, M. T., Qin, H., Moghaddam, N. S., Ibrahim, H., Jahadakbar, A., ... Ye, C. (2017). Improving surface finish and wear resistance of additive manufactured nickel-titanium by ultrasonic nano-crystal surface modification. Journal of Materials Processing Technology, 249(June), 433-440. https://doi.org/10.1016/j.jmatprotec.2017.06.038

Ma, C. P., Guan, Y. C., \& Zhou, W. (2017). Laser polishing of additive manufactured Ti alloys. Optics and Lasers in Engineering, 93(January), 171-177. https://doi.org/10.1016/j.optlaseng.2017.02.005

Mercelis, P., \& Kruth, J. (2006). Residual stresses in selective laser sintering and selective laser melting. Rapid Prototyping Journal, 12(5), 254-265. https://doi.org/10.1108/13552540610707013

Merkt, S., Hinke, C., Bültmann, J., Brandt, M., \& Xie, Y. M. (2015). Mechanical response of TiAl6V4 lattice structures manufactured by selective laser melting in quasistatic and dynamic compression tests. Journal of Laser Applications, 27(S1), S17006. https://doi.org/10.2351/1.4898835

Mohammadian, N., Turenne, S., \& Brailovski, V. (2018). Surface finish control of additively-manufactured Inconel 625 components using combined chemicalabrasive flow polishing. Journal of Materials Processing Technology, 252, 728738. https://doi.org/10.1016/j.jmatprotec.2017.10.020

Mukherjee, T., Zhang, W., \& DebRoy, T. (2017). An improved prediction of residual stresses and distortion in additive manufacturing. Computational Materials Science, 126, 360-372. https://doi.org/10.1016/j.commatsci.2016.10.003 
Mukherjee, T., Zuback, J. S., De, A., \& DebRoy, T. (2016). Printability of alloys for additive manufacturing. Scientific Reports, 6(1), 19717. https://doi.org/10.1038/srep19717

Murr, L. E., Gaytan, S. M., Martinez, E., Medina, F., \& Wicker, R. B. (2012). Next generation orthopaedic implants by additive manufacturing using electron beam melting. International Journal of Biomaterials, 2012. https://doi.org/10.1155/2012/245727

Rotty, C., Mandroyan, A., Doche, M. L., \& Hihn, J. Y. (2016). Electropolishing of CuZn brasses and 316L stainless steels: Influence of alloy composition or preparation process (ALM vs. standard method). Surface and Coatings Technology, 307, 125135. https://doi.org/10.1016/j.surfcoat.2016.08.076

Schmidt, M., Merklein, M., Bourell, D., Dimitrov, D., Hausotte, T., Wegener, K., ... Levy, G. N. (2017). Laser based additive manufacturing in industry and academia. CIRP Annals - Manufacturing Technology, 66(2), 561-583. https://doi.org/10.1016/j.cirp.2017.05.011

Shi, Q., Gu, D., Xia, M., Cao, S., \& Rong, T. (2016). Effects of laser processing parameters on thermal behavior and melting/solidification mechanism during selective laser melting of TiC/Inconel 718 composites. Optics \& Laser Technology, 84, 9-22. https://doi.org/10.1016/j.optlastec.2016.04.009

Slotwinski, J. A., Garboczi, E. J., \& Hebenstreit, K. M. (2014). Porosity Measurements and Analysis for Metal Additive Manufacturing Process Control. Journal of Research of the National Institute of Standards and Technology, 119, 494. 
https://doi.org/10.6028/jres.119.019

Strano, G., Hao, L., Everson, R. M., \& Evans, K. E. (2013). Surface roughness analysis, modelling and prediction in selective laser melting. Journal of Materials Processing Technology, 213(4), 589-597. https://doi.org/10.1016/j.jmatprotec.2012.11.011

Sufiiarov, V. S., Popovich, A. A., Borisov, E. V., Polozov, I. A., Masaylo, D. V., \& Orlov, A. V. (2017). The Effect of Layer Thickness at Selective Laser Melting. Procedia Engineering, 174, 126-134. https://doi.org/10.1016/j.proeng.2017.01.179

Tammas-Williams, S., Withers, P. J., Todd, I., \& Prangnell, P. B. (2016). Porosity regrowth during heat treatment of hot isostatically pressed additively manufactured titanium components. Scripta Materialia, 122, 72-76.

https://doi.org/10.1016/j.scriptamat.2016.05.002

Thijs, L., Verhaeghe, F., Craeghs, T., Humbeeck, J. Van, \& Kruth, J. P. (2010). A study of the microstructural evolution during selective laser melting of Ti-6Al-4V. Acta Materialia, 58(9), 3303-3312. https://doi.org/10.1016/j.actamat.2010.02.004

Thomas, D. (2016). Costs, benefits, and adoption of additive manufacturing: a supply chain perspective. International Journal of Advanced Manufacturing Technology, 85(5-8), 1857-1876. https://doi.org/10.1007/s00170-015-7973-6

ur Rahman, Z., Deen, K. M., Cano, L., \& Haider, W. (2017). The effects of parametric changes in electropolishing process on surface properties of $316 \mathrm{~L}$ stainless steel. Applied Surface Science, 410, 432-444. https://doi.org/10.1016/j.apsusc.2017.03.081 
Urlea, V., \& Brailovski, V. (2017). Electropolishing and electropolishing-related allowances for powder bed selectively laser-melted Ti-6Al-4V alloy components. Journal of Materials Processing Technology, 242, 1-11. https://doi.org/10.1016/j.jmatprotec.2016.11.014

Wang, Z., Denlinger, E., Michaleris, P., Stoica, A. D., Ma, D., \& Beese, A. M. (2017). Residual stress mapping in Inconel 625 fabricated through additive manufacturing: Method for neutron diffraction measurements to validate thermomechanical model predictions. Materials and Design, 113, 169-177. https://doi.org/10.1016/j.matdes.2016.10.003

Wei, H. L., Elmer, J. W., \& DebRoy, T. (2017). Three-dimensional modeling of grain structure evolution during welding of an aluminum alloy. Acta Materialia, 126, 413-425. https://doi.org/10.1016/j.actamat.2016.12.073

Willenborg, E. (2011). Tailored Light 2. (R. Poprawe, Ed.). Berlin, Heidelberg: Springer Berlin Heidelberg. https://doi.org/10.1007/978-3-642-01237-2

Zheng, X., Lee, H., Weisgraber, T. H., Shusteff, M., DeOtte, J., Duoss, E. B., ... Spadaccini, C. M. (2014). Ultralight, ultrastiff mechanical metamaterials. Science, 344(6190), 1373-1377. https://doi.org/10.1126/science.1252291 


\section{Appendices}

\section{A. Drawings}
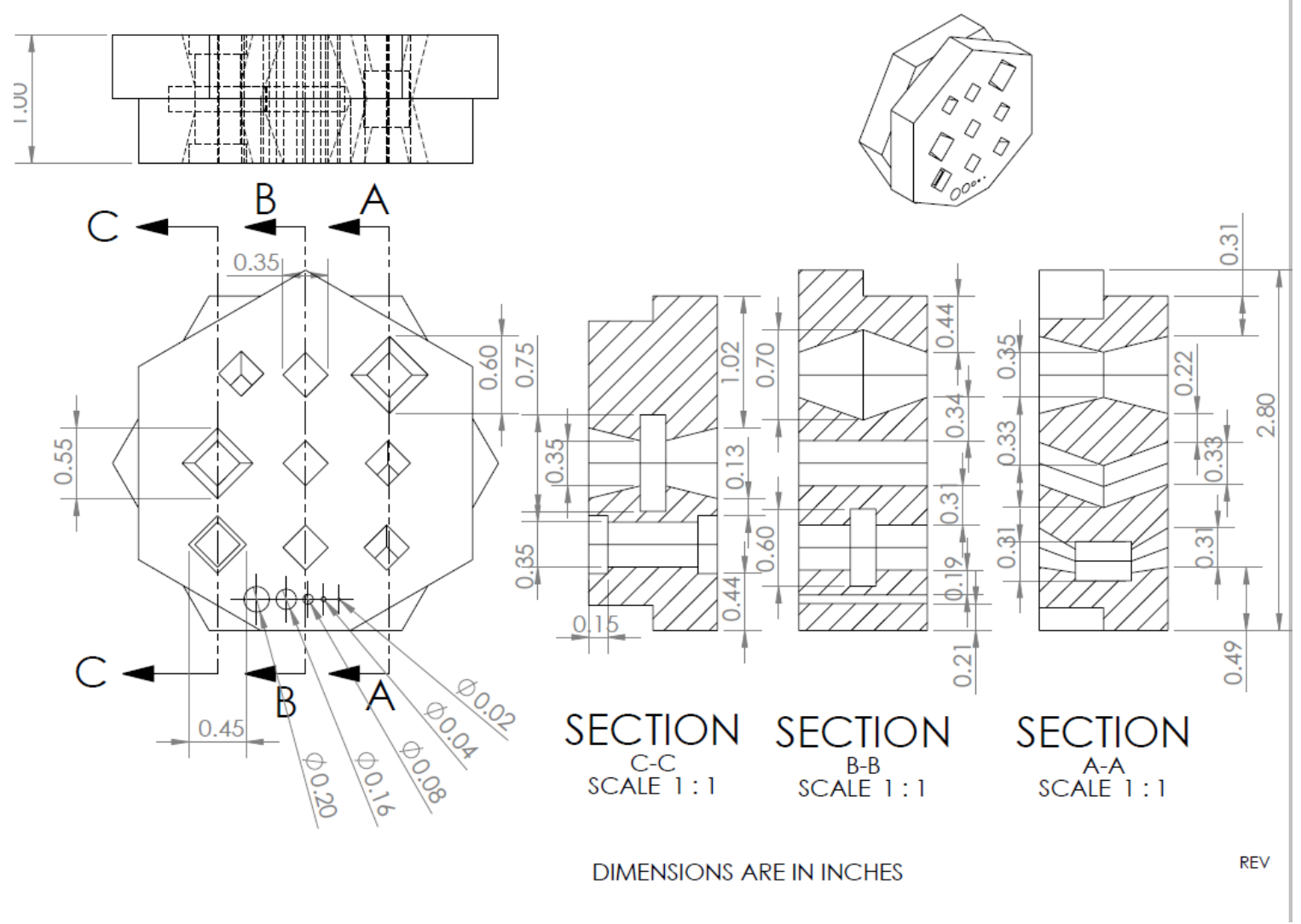


\section{B. SLM Laser Parameters}

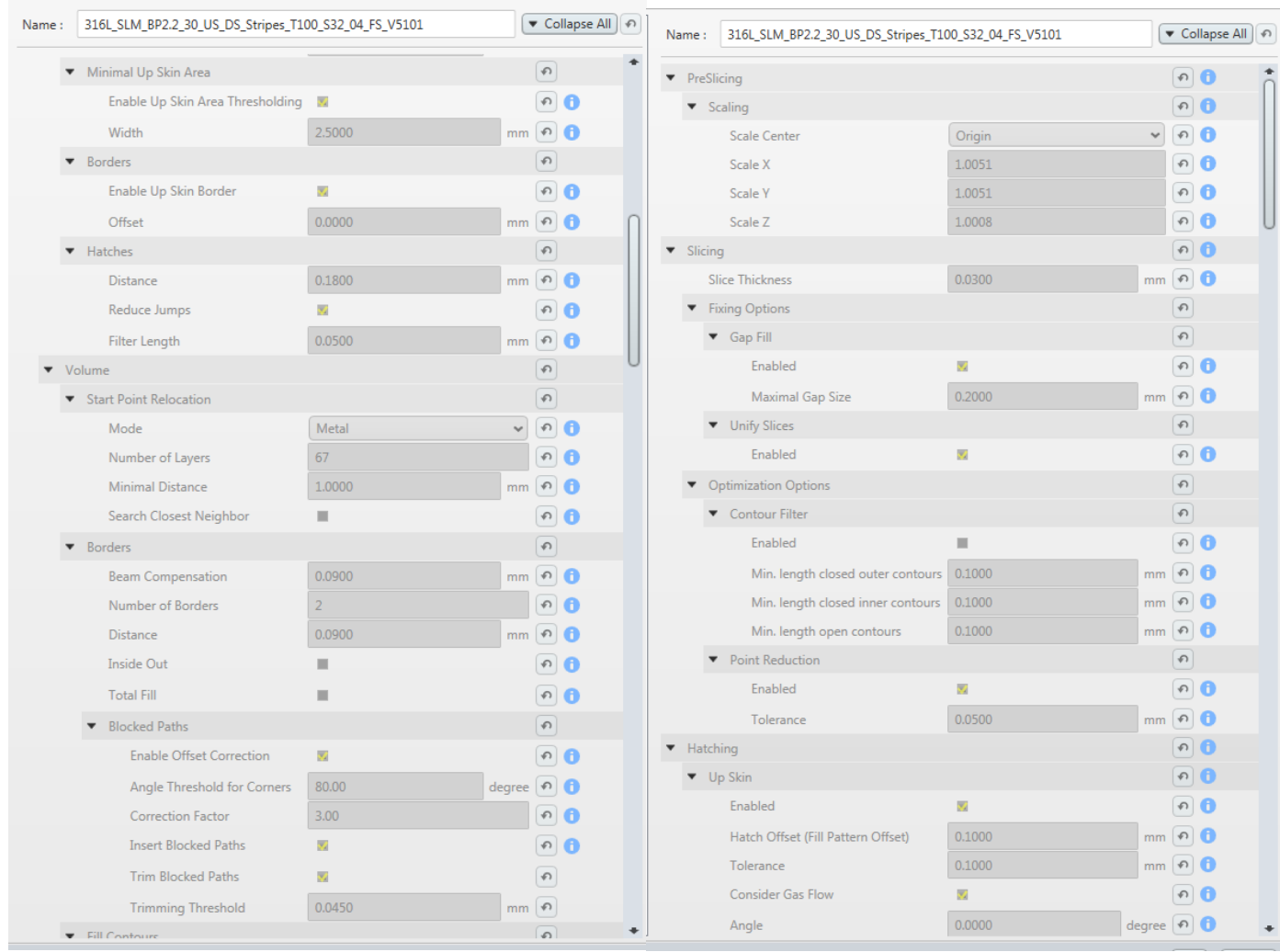

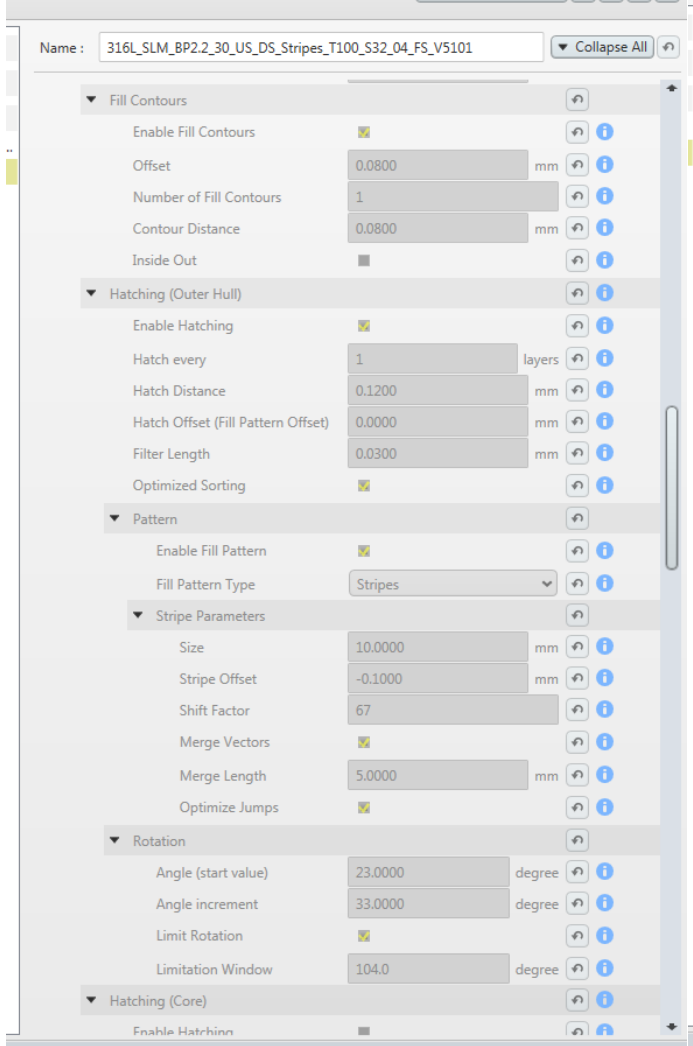

\begin{tabular}{|c|c|c|c|c|c|}
\hline \multirow[t]{6}{*}{ Name: } & \multicolumn{3}{|c|}{ 316L_SLM_BP2.2_30_US_DS_Stripes_T100_S32_04_FS_V5101 } & \multicolumn{2}{|c|}{ - Collapse All $\curvearrowleft$} \\
\hline & Enable Hatching & = & & ค 0 & \\
\hline & - Transition Contour & & & n & \\
\hline & Transition Contour Offset & 0.2500 & $\mathrm{~mm}$ & ( ) 0 & \\
\hline & Number of Contours & 1 & & ค 0 & \\
\hline & Distance & 1.0000 & $\mathrm{~mm}$ & ค 0 & \\
\hline \multicolumn{2}{|c|}{ Down Skin } & & & ค 0 & \\
\hline \multicolumn{2}{|r|}{ Enabled } & $\bar{a}$ & & ค 0 & \\
\hline \multicolumn{2}{|r|}{ Hatch Offset (Fill Pattern Offset) } & 0.0800 & $\mathrm{~mm}$ & ก 0 & \\
\hline \multicolumn{2}{|r|}{ Layer Reference } & 2 & Number of layers & ๑ 0 & \\
\hline \multicolumn{2}{|r|}{ Maximal Surface Angle } & 69.0000 & degree & ( 0 & \\
\hline \multicolumn{2}{|r|}{ Consider Gas Flow } & $\mathbf{a}$ & & ค 0 & \\
\hline \multicolumn{2}{|r|}{ Angle } & 67.0000 & degree & ๑ 0 & \\
\hline \multicolumn{2}{|r|}{ Increment Angle } & 66.0000 & degree & ๑ & \\
\hline \multicolumn{2}{|r|}{ - Hatches } & & & n & \\
\hline \multicolumn{2}{|r|}{ Distance } & 0.0600 & $\mathrm{~mm}$ & ( 0 & \\
\hline \multicolumn{2}{|r|}{ Reduce Jumps } & $\bar{a}$ & & ค 0 & \\
\hline \multicolumn{2}{|r|}{ Filter Length } & 0.0600 & $\mathrm{~mm}$ & ค 0 & \\
\hline \multicolumn{2}{|r|}{ Overlap with Volume Area } & 0.0600 & $\mathrm{~mm}$ & ค 0 & \\
\hline \multicolumn{2}{|r|}{ - Pattern } & & & ค & \\
\hline \multicolumn{2}{|r|}{ Enable Fill Pattern } & $\mathbf{x}$ & & ค 0 & \\
\hline \multicolumn{2}{|r|}{ Fill Pattern Type } & Stripes & $\sim$ & ค 0 & \\
\hline \multicolumn{2}{|r|}{ - Stripe Parameters } & & & n & \\
\hline \multicolumn{2}{|r|}{ Size } & 10.0000 & $\mathrm{~mm}$ & ( 0 & \\
\hline \multicolumn{2}{|r|}{ Stripe Offset } & 0.0000 & $\mathrm{~mm}$ & ค 0 & \\
\hline \multicolumn{2}{|r|}{ Shift Factor } & 67 & & ค 0 & \\
\hline \multicolumn{2}{|r|}{ Merge Vectors } & $\mathbf{a}$ & & ค 0 & \\
\hline \multicolumn{2}{|r|}{ Merge Length } & 5.0000 & $\mathrm{~mm}$ & ค 0 & \\
\hline \multicolumn{2}{|r|}{ Optimize Jumps } & $\bar{a}$ & & ค 1 & \\
\hline \multicolumn{2}{|c|}{ - Scanning } & & & ค (1) & \\
\hline \multicolumn{2}{|c|}{ - Blocked Paths } & & & ค & + \\
\hline
\end{tabular}




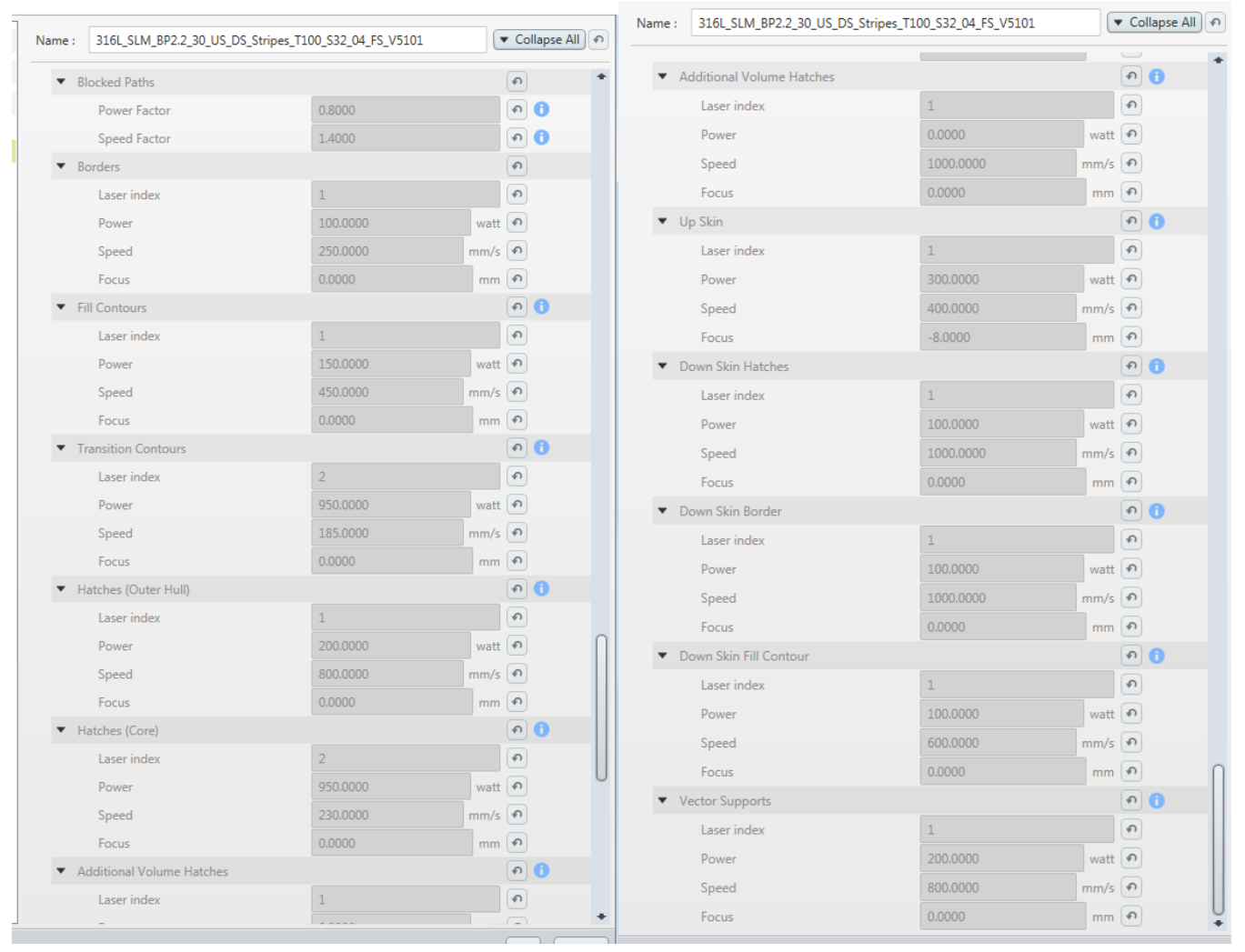




\section{Raw Measurement Data}

\section{Exterior Surface Roughness Measurements}

\begin{tabular}{|c|c|c|c|c|c|}
\hline Sample & Location & $\mathrm{Ra}$ (micron) & $\mathrm{Rq}$ (micron) & $\begin{array}{l}\mathrm{Rz} \\
\text { (micron) }\end{array}$ & Parameters \\
\hline EP & Surface 1A & 3.18 & 4.14 & 17.36 & $0.8 \times 5$ \\
\hline EP & $1 \mathrm{~A}$ & 3.47 & 4.62 & 19.44 & $0.8 \times 5$ \\
\hline EP & $1 \mathrm{~A}$ & 5.79 & 7.23 & 29.57 & $0.8 \times 5$ \\
\hline EP & Surface 1B & 4.28 & 5 & 17.66 & $0.8 \times 5$ \\
\hline EP & 1B & 9.81 & 12.19 & 44.14 & $0.8 \times 5$ \\
\hline$\overline{E P}$ & $1 \mathrm{~B}$ & 7.38 & 9.06 & 33.92 & $0.8 \times 5$ \\
\hline EP & Surface $2 \mathrm{~A}$ & 2.35 & 3 & 12.55 & $0.8 \times 5$ \\
\hline EP & $2 \mathrm{~A}$ & 4.66 & 6.07 & 25.17 & $0.8 \times 5$ \\
\hline EP & $2 \mathrm{~A}$ & 4.21 & 5.19 & 21 & $0.8 \times 5$ \\
\hline EP & Surface 2B & 3.14 & 3.99 & 15.52 & $0.8 \times 5$ \\
\hline EP & $2 \mathrm{~B}$ & 3.41 & 4.35 & 17.45 & $0.8 \times 5$ \\
\hline EP & $2 \mathrm{~B}$ & 3.44 & 4.27 & 17.43 & $0.8 \times 5$ \\
\hline EP & Surface 5 & 3.02 & 2.33 & 7.58 & $0.8 \times 5$ \\
\hline EP & 5 & 1.93 & 2.24 & 7.04 & $0.8 \times 5$ \\
\hline EP & 5 & 1.63 & 1.95 & 5.79 & $0.8 \times 5$ \\
\hline EP & Surface $6 \mathrm{~A}$ & 3.59 & 4.4 & 17.2 & $0.8 \times 5$ \\
\hline EP & $6 \mathrm{~A}$ & 2.22 & 2.7 & 11.62 & $0.8 \times 5$ \\
\hline EP & $6 \mathrm{~A}$ & 3.73 & 4.9 & 21.27 & $0.8 \times 5$ \\
\hline EP & Surface 6B & 3.71 & 4.48 & 17.55 & $0.8 \times 5$ \\
\hline EP & $6 \mathrm{~B}$ & 3.84 & 4.84 & 19.29 & $0.8 \times 5$ \\
\hline EP & $6 \mathrm{~B}$ & 3.71 & 4.7 & 18.16 & $0.8 \times 5$ \\
\hline EP & Surface 8 bot & 2.9 & $\mathrm{x}$ & 17.07 & 2.5 \\
\hline EP & Surface 8 bot & 1.28 & $\mathrm{x}$ & 6.37 & 0.8 \\
\hline EP & Surface 8 bot & 0.48 & $\mathrm{x}$ & 2.07 & 0.25 \\
\hline EP & Surface $8 \mathrm{Mid}$ & 3.13 & $\mathrm{x}$ & 18.98 & 2.5 \\
\hline EP & Surface $8 \mathrm{Mid}$ & 3.08 & $\mathrm{x}$ & 15.66 & 0.8 \\
\hline EP & Surface $8 \mathrm{Mid}$ & 2.1 & $\mathrm{x}$ & 9.64 & 0.25 \\
\hline EP & Surface 8 Top & 3.54 & $\mathrm{x}$ & 18.61 & 2.5 \\
\hline EP & Surface 8 Top & 2.07 & $\mathrm{x}$ & 10.97 & 0.8 \\
\hline EP & Surface 8 Top & 0.56 & $\mathrm{x}$ & 2.26 & 0.25 \\
\hline EP & Surface 8 Left & 3.97 & $\mathrm{x}$ & 23.56 & 2.5 \\
\hline EP & Surface 8 Left & 2.88 & $\mathrm{x}$ & 13.81 & 0.8 \\
\hline EP & Surface 8 Left & 1.07 & $\mathrm{x}$ & 3.91 & 0.25 \\
\hline EP & $\begin{array}{l}\text { Surface } 8 \\
\text { Middle }\end{array}$ & 4.87 & $\mathrm{x}$ & 30.23 & 2.5 \\
\hline
\end{tabular}




\begin{tabular}{|c|c|c|c|c|c|}
\hline EP & $\begin{array}{l}\text { Surface } 8 \\
\text { Middle }\end{array}$ & 3.35 & $\mathrm{x}$ & 17.67 & 0.8 \\
\hline EP & $\begin{array}{l}\text { Surface } 8 \\
\text { Middle }\end{array}$ & 1.03 & $\mathrm{x}$ & 4.46 & 0.25 \\
\hline EP & $\begin{array}{l}\text { Surface } 8 \\
\text { Right }\end{array}$ & 4.72 & $x$ & 29.45 & 2.5 \\
\hline EP & $\begin{array}{l}\text { Surface } 8 \\
\text { Right }\end{array}$ & 4.68 & $\mathrm{x}$ & 24.88 & 0.8 \\
\hline EP & $\begin{array}{l}\text { Surface } 8 \\
\text { Right }\end{array}$ & 1.99 & $\mathrm{x}$ & 7.68 & 0.25 \\
\hline AP & Surface $1 \mathrm{~A}$ & 11.86 & & & \\
\hline AP & $1 \mathrm{~A}$ & 13.22 & & & \\
\hline AP & $1 \mathrm{~A}$ & 11.92 & & & \\
\hline AP & Surface 1B & 9.66 & & & \\
\hline AP & 1B & 9.62 & & & \\
\hline AP & 1B & 10 & & & \\
\hline $\mathrm{AP}$ & Surface $2 \mathrm{~A}$ & 7.59 & & & \\
\hline AP & $2 \mathrm{~A}$ & 7.86 & & & \\
\hline AP & $2 \mathrm{~A}$ & 8.17 & & & \\
\hline AP & Surface 2B & 8.48 & & & \\
\hline $\mathrm{AP}$ & $2 \mathrm{~B}$ & 7.9 & & & \\
\hline AP & $2 \mathrm{~B}$ & 8 & & & \\
\hline AP & Surface 5 & 10.03 & & & \\
\hline AP & 5 & 8.14 & & & \\
\hline AP & 5 & 6.54 & & & \\
\hline AP & Surface $6 \mathrm{~A}$ & 7.52 & & & \\
\hline AP & $6 \mathrm{~A}$ & 6.83 & & & \\
\hline AP & $6 \mathrm{~A}$ & 7.24 & & & \\
\hline $\mathrm{AP}$ & Surface 6B & 5.71 & & & \\
\hline AP & $6 B$ & 6.19 & & & \\
\hline AP & $6 B$ & 10.42 & & & \\
\hline
\end{tabular}

Interior Surface Roughness Measurements

\begin{tabular}{|l|l|l|l|l|l|}
\hline Sample & Location & $\mathrm{Ra}$ (micron) & $\mathrm{Rz}$ (micron) & $\begin{array}{l}\text { Rq } \\
\text { (micron) }\end{array}$ & Parameters \\
\hline EP & $1-1$ Interior & 18.08 & 88.21 & 23.06 & $0.8 \times 5$ \\
\hline EP & $1-1$ Interior & 4.49 & 20.8 & 5.26 & $0.8 \times 5$ \\
\hline EP & $1-1$ Interior & 9.09 & 61.06 & 12.78 & $0.8 \times 5$ \\
\hline EP & $1-2$ Interior & 7.5 & 41.15 & 9.42 & $0.8 \times 5$ \\
\hline EP & $1-2$ Interior & 8.22 & 43.89 & 10.15 & $0.8 \times 5$ \\
\hline EP & $1-2$ Interior & 10.92 & 64.08 & 13.53 & $0.8 \times 5$ \\
\hline EP & $1-3$ Interior & 14.32 & 66.87 & 17.41 & $0.8 \times 5$ \\
\hline EP & $1-3$ Interior & 15.51 & 71.3 & 18.54 & $0.8 \times 5$ \\
\hline
\end{tabular}




\begin{tabular}{|c|c|c|c|c|c|}
\hline EP & 1-3 Interior & 16.99 & 84.97 & 20.6 & $0.8 \times 5$ \\
\hline EP & 1-4 Interior & 17.27 & 85.42 & 21.3 & $0.8 \times 5$ \\
\hline EP & 1-4 Interior & 17.61 & 88.85 & 21.44 & $0.8 \times 5$ \\
\hline EP & 1-4 Interior & 16.89 & 84.89 & 20.33 & $0.8 \times 5$ \\
\hline EP & 2-1 Interior & 6.89 & 32.23 & 8.49 & $0.25 \times 5$ \\
\hline EP & 2-1 Interior & 3.38 & 15.93 & 4.14 & $0.25 \times 5$ \\
\hline EP & 2-1 Interior & 0.91 & 3.4 & 1.12 & $0.25 \times 5$ \\
\hline EP & 2-2 Interior & 3.13 & 15.03 & 3.9 & $0.25 \times 5$ \\
\hline EP & 2-2 Interior & 1.15 & 4.86 & 1.35 & $0.25 \times 5$ \\
\hline EP & 2-2 Interior & 4.61 & 23.44 & 6.05 & $0.25 \times 5$ \\
\hline EP & 2-3 Interior & 6.79 & 31.81 & 8.16 & $0.25 \times 5$ \\
\hline EP & 2-3 Interior & 9.12 & 39.89 & 10.88 & $0.25 \times 5$ \\
\hline EP & 2-3 Interior & 5.54 & 24.26 & 6.86 & $0.25 \times 5$ \\
\hline EP & 2-4 Interior & 7.8 & 35.54 & 9.48 & $0.25 \times 5$ \\
\hline EP & 2-4 Interior & 6.11 & 29.26 & 7.53 & $0.25 \times 5$ \\
\hline EP & 2-4 Interior & 6.45 & 28.32 & 7.88 & $0.25 \times 5$ \\
\hline EP & 3-1 Interior & 7.79 & 37.16 & 9.16 & $0.8 \times 5$ \\
\hline EP & 3-1 Interior & 5.29 & 24.96 & 6.62 & $0.8 \times 5$ \\
\hline EP & 3-1 Interior & 5.64 & 27.22 & 7.03 & $0.8 \times 5$ \\
\hline EP & 3-2 Interior & 2.4 & 19.9 & 3.75 & $0.8 \times 5$ \\
\hline EP & 3-2 Interior & 5.76 & 39.26 & 8.26 & $0.8 \times 5$ \\
\hline EP & 3-2 Interior & 5.36 & 28 & 6.88 & $0.8 \times 5$ \\
\hline EP & 3-3 Interior & 8.16 & 46.77 & 10.48 & $0.8 \times 5$ \\
\hline EP & 3-3 Interior & 11.64 & 59.91 & 14.51 & $0.8 \times 5$ \\
\hline EP & 3-3 Interior & 10.52 & 51.39 & 12.99 & $0.8 \times 5$ \\
\hline EP & 3-4 Interior & 13.65 & 63.35 & 16.16 & $0.8 \times 5$ \\
\hline EP & 3-4 Interior & 16.43 & 73.17 & 19.7 & $0.8 \times 5$ \\
\hline EP & 3-4 Interior & 19.55 & 89.87 & 23.12 & $0.8 \times 5$ \\
\hline EP & 4-1 Interior & 9.96 & 53.81 & 12.96 & $0.8 \times 5$ \\
\hline EP & 4-1 Interior & 7.05 & 44.28 & 9.29 & $0.8 \times 5$ \\
\hline EP & 4-1 Interior & 8.6 & 44.93 & 11.07 & $0.8 \times 5$ \\
\hline EP & 4-2 Interior & 5.79 & 28.86 & 7.15 & $0.8 \times 5$ \\
\hline EP & 4-2 Interior & 7.93 & 39.87 & 9.98 & $0.8 \times 5$ \\
\hline EP & 4-2 Interior & 6.4 & 41.44 & 8.45 & $0.8 \times 5$ \\
\hline EP & 4-3 Interior & 12.99 & 77.1 & 17.35 & $0.8 \times 5$ \\
\hline EP & 4-3 Interior & 19.61 & 94.58 & 23.8 & $0.8 \times 5$ \\
\hline EP & 4-3 Interior & 14.34 & 77.46 & 18.12 & $0.8 \times 5$ \\
\hline EP & 4-4 Interior & 15.34 & 73.7 & 18.29 & $0.8 \times 5$ \\
\hline EP & 4-4 Interior & 18.09 & 92.26 & 22.04 & $0.8 \times 5$ \\
\hline EP & 4-4 Interior & 15.42 & 72.94 & 18.7 & $0.8 \times 5$ \\
\hline EP & 5-1 Interior & 10.73 & 55 & 13.35 & $0.8 \times 5$ \\
\hline
\end{tabular}




\begin{tabular}{|c|c|c|c|c|c|}
\hline EP & 5-1 Interior & 11.88 & 62.07 & 14.88 & $0.8 \times 5$ \\
\hline EP & 5-1 Interior & 11.61 & 59.91 & 14.36 & $0.8 \times 5$ \\
\hline EP & 5-2 Interior & 5 & 26 & 6.29 & $0.8 \times 5$ \\
\hline EP & 5-2 Interior & 5.22 & 33.04 & 7.69 & $0.8 \times 5$ \\
\hline EP & 5-2 Interior & 6.06 & 34.91 & 7.95 & $0.8 \times 5$ \\
\hline EP & 5-3 Interior & 11 & 51.14 & 13.11 & $0.8 \times 5$ \\
\hline EP & 5-3 Interior & 14.35 & 73.93 & 17.8 & $0.8 \times 5$ \\
\hline EP & 5-3 Interior & 14.74 & 74.79 & 17.88 & $0.8 \times 5$ \\
\hline EP & 5-4 Interior & 17.68 & 90.84 & 21.81 & $0.8 \times 5$ \\
\hline EP & 5-4 Interior & 18.63 & 95.5 & 22.71 & $0.8 \times 5$ \\
\hline EP & 5-4 Interior & 17.59 & 90.72 & 21.86 & $0.8 \times 5$ \\
\hline EP & 6-1 Interior & 10.27 & 54.56 & 12.47 & $0.8 \times 5$ \\
\hline EP & 6-1 Interior & 6.88 & 29.92 & 8.22 & $0.8 \times 5$ \\
\hline EP & 6-1 Interior & 5.76 & 27.83 & 7.17 & $0.8 \times 5$ \\
\hline EP & 6-2 Interior & 6.65 & 34.88 & 8.38 & $0.8 \times 5$ \\
\hline EP & 6-2 Interior & 5.23 & 29.03 & 6.62 & $0.8 \times 5$ \\
\hline EP & 6-2 Interior & 5.05 & 31.87 & 6.76 & $0.8 \times 5$ \\
\hline EP & 6-3 Interior & 15.57 & 70.14 & 19.58 & $0.8 \times 5$ \\
\hline EP & 6-3 Interior & 17.72 & 80.96 & 20.8 & $0.8 \times 5$ \\
\hline EP & 6-3 Interior & 14.63 & 72.33 & 17.99 & $0.8 \times 5$ \\
\hline EP & 6-4 Interior & 16.9 & 86.18 & 21.11 & $0.8 \times 5$ \\
\hline EP & 6-4 Interior & 15.07 & 72.25 & 18.15 & $0.8 \times 5$ \\
\hline EP & 6-4 Interior & 14.61 & 77.52 & 18.26 & $0.8 \times 5$ \\
\hline EP & 7-1 Interior & 6.46 & 43.11 & 8.28 & $0.8 \times 5$ \\
\hline EP & 7-1 Interior & 7.82 & 45.31 & 10.04 & $0.8 \times 5$ \\
\hline EP & 7-1 Interior & 4.96 & 27.43 & 6.2 & $0.8 \times 5$ \\
\hline EP & 7-2 Interior & 6.12 & 38.57 & 8.57 & $0.8 \times 5$ \\
\hline EP & 7-2 Interior & 6.33 & 33.47 & 7.87 & $0.8 \times 5$ \\
\hline EP & 7-2 Interior & 11.21 & 56.76 & 14.24 & $0.8 \times 5$ \\
\hline EP & 7-3 Interior & 10.29 & 48.22 & 12.64 & $0.8 \times 5$ \\
\hline EP & 7-3 Interior & 11.9 & 58.02 & 14.33 & $0.8 \times 5$ \\
\hline EP & 7-3 Interior & 11.53 & 56.24 & 14 & $0.8 \times 5$ \\
\hline EP & 7-4 Interior & 11.79 & 61.86 & 14.33 & $0.8 \times 5$ \\
\hline EP & 7-4 Interior & 13 & 63.03 & 15.7 & $0.8 \times 5$ \\
\hline EP & 7-4 Interior & 12.53 & 58.87 & 14.99 & $0.8 \times 5$ \\
\hline EP & 8-1 Interior & 2.84 & 13.22 & 3.52 & $0.8 \times 5$ \\
\hline EP & 8-1 Interior & 4.55 & 20.9 & 5.54 & $0.8 \times 5$ \\
\hline EP & 8-1 Interior & 7.25 & 37.47 & 9.04 & $0.8 \times 5$ \\
\hline EP & 8-2 Interior & 5.82 & 32.18 & 7.43 & $0.8 \times 5$ \\
\hline EP & 8-2 Interior & 7.65 & 36.69 & 9.32 & $0.8 \times 5$ \\
\hline EP & 8-2 Interior & 3.99 & 19.18 & 4.93 & $0.8 \times 5$ \\
\hline
\end{tabular}




\begin{tabular}{|c|c|c|c|c|c|}
\hline EP & 8-3 Interior & 13.84 & 70.39 & 17.31 & $0.8 \times 5$ \\
\hline EP & 8-3 Interior & 16.77 & 82.48 & 19.83 & $0.8 \times 5$ \\
\hline EP & 8-3 Interior & 22.67 & 113.2 & 27.77 & $0.8 \times 5$ \\
\hline EP & 8-4 Interior & 20.07 & 88.85 & 23.45 & $0.8 \times 5$ \\
\hline EP & 8-4 Interior & 13.8 & 77.58 & 17.38 & $0.8 \times 5$ \\
\hline EP & 8-4 Interior & 14.39 & 74.81 & 18.03 & $0.8 \times 5$ \\
\hline EP & 9-1 Interior & 7.74 & 37.43 & 9.4 & $0.8 \times 5$ \\
\hline EP & 9-1 Interior & 5.65 & 32.69 & 7.43 & $0.8 \times 5$ \\
\hline EP & 9-1 Interior & 6.46 & 29.83 & 7.61 & $0.8 \times 5$ \\
\hline EP & 9-2 Interior & 9.47 & 49.74 & 11.94 & $0.8 \times 5$ \\
\hline EP & 9-2 Interior & 5.63 & 25.08 & 6.85 & $0.8 \times 5$ \\
\hline EP & 9-2 Interior & 4.77 & 33.92 & 6.2 & $0.8 \times 5$ \\
\hline EP & 9-3 Interior & 15.52 & 83.38 & 19.03 & $0.8 \times 5$ \\
\hline EP & 9-3 Interior & 13.82 & 69.9 & 16.86 & $0.8 \times 5$ \\
\hline EP & 9-3 Interior & 14.54 & 67.05 & 17.72 & $0.8 \times 5$ \\
\hline EP & 9-4 Interior & 22.48 & 114.9 & 27.7 & $0.8 \times 5$ \\
\hline EP & 9-4 Interior & 17.51 & 91.62 & 21.23 & $0.8 \times 5$ \\
\hline EP & 9-4 Interior & 17.52 & 85.98 & 21.55 & $0.8 \times 5$ \\
\hline EP & Big O Top & 22.24 & 96.67 & 26.07 & $0.8 \times 5$ \\
\hline EP & Big O Top & 22.66 & 110.2 & 27.48 & $0.8 \times 5$ \\
\hline EP & Big O Top & 24.34 & 111.4 & 29.01 & $0.8 \times 5$ \\
\hline EP & Big O Bot & 5.49 & 31.16 & 7.1 & $0.8 \times 5$ \\
\hline EP & Big O Bot & 6.8 & 34.56 & 8.45 & $0.8 \times 5$ \\
\hline EP & Big O Bot & 9.36 & 48.27 & 11.76 & $0.8 \times 5$ \\
\hline EP & Small O Top & 19.54 & 92.72 & 22.89 & $0.8 \times 5$ \\
\hline EP & Small O Top & 18.37 & 95.87 & 22.46 & $0.8 \times 5$ \\
\hline EP & Small O Top & 17.85 & 90.47 & 21.99 & $0.8 \times 5$ \\
\hline EP & Small O bot & 4.19 & 23.52 & 5.18 & $0.8 \times 5$ \\
\hline EP & Small O bot & 5.65 & 30.89 & 7.07 & $0.8 \times 5$ \\
\hline EP & Small O bot & 4.81 & 24.42 & 5.71 & $0.8 \times 5$ \\
\hline AP & 1-1 Interior & 7.82 & 34.6 & 9.23 & $0.25 \times 5$ \\
\hline AP & 1-1 Interior & 5.74 & 28.55 & 7.35 & $0.25 \times 5$ \\
\hline $\mathrm{AP}$ & 1-1 Interior & 5.67 & 24.5 & 6.91 & $0.25 \times 5$ \\
\hline AP & 1-2 Interior & 9.22 & 35.27 & 10.76 & $0.25 \times 5$ \\
\hline AP & 1-2 Interior & 5.06 & 25.08 & 6.16 & $0.25 \times 5$ \\
\hline AP & 1-2 Interior & 4.39 & 19.89 & 5.51 & $0.25 \times 5$ \\
\hline $\mathrm{AP}$ & 1-3 Interior & 8.1 & 35.64 & 9.68 & $0.25 \times 5$ \\
\hline $\mathrm{AP}$ & 1-3 Interior & 6.83 & 34.16 & 8.46 & $0.25 \times 5$ \\
\hline AP & 1-3 Interior & 7.89 & 33.05 & 9.15 & $0.25 \times 5$ \\
\hline AP & 1-4 Interior & 8.49 & 39.22 & 10.21 & $0.25 \times 5$ \\
\hline $\mathrm{AP}$ & 1-4 Interior & 5.67 & 26.03 & 7.07 & $0.25 \times 5$ \\
\hline
\end{tabular}




\begin{tabular}{|c|c|c|c|c|c|}
\hline $\mathrm{AP}$ & 1-4 Interior & 7.95 & 35.82 & 9.45 & $0.25 \times 5$ \\
\hline $\mathrm{AP}$ & 2-1 Interior & 11.58 & 66.08 & 14.67 & \\
\hline $\mathrm{AP}$ & 2-1 Interior & 13.22 & 68.84 & 16.4 & \\
\hline $\mathrm{AP}$ & 2-1 Interior & 12.79 & 74.16 & 16.94 & \\
\hline $\mathrm{AP}$ & 2-2 Interior & 15.17 & 73.68 & 18.26 & \\
\hline $\mathrm{AP}$ & 2-2 Interior & 15.07 & 65.43 & 17.53 & \\
\hline $\mathrm{AP}$ & 2-2 Interior & 11.54 & 59.65 & 14.28 & \\
\hline $\mathrm{AP}$ & 2-3 Interior & 13.1 & 80.46 & 16.49 & \\
\hline$\overline{\mathrm{AP}}$ & 2-3 Interior & 14.38 & 77.28 & 17.72 & \\
\hline $\mathrm{AP}$ & 2-3 Interior & 14.77 & 74.55 & 17.87 & \\
\hline $\mathrm{AP}$ & 2-4 Interior & 13.99 & 75.04 & 17.19 & \\
\hline $\mathrm{AP}$ & 2-4 Interior & 13.57 & 78.15 & 17.03 & \\
\hline $\mathrm{AP}$ & 2-4 Interior & 15.88 & 76.66 & 19.13 & \\
\hline $\mathrm{AP}$ & 3-1 Interior & 14.3 & 68.89 & 17.23 & \\
\hline $\mathrm{AP}$ & 3-1 Interior & 9.64 & 52.35 & 12.21 & \\
\hline $\mathrm{AP}$ & 3-1 Interior & 14.56 & 72.73 & 17.68 & \\
\hline $\mathrm{AP}$ & 3-2 Interior & 9.79 & 41.35 & 11.46 & \\
\hline $\mathrm{AP}$ & 3-2 Interior & 5.13 & 24.79 & 6.49 & \\
\hline $\mathrm{AP}$ & 3-2 Interior & 7.09 & 31.08 & 8.39 & \\
\hline $\mathrm{AP}$ & 3-3 Interior & 14.93 & 75.09 & 18.26 & \\
\hline $\mathrm{AP}$ & 3-3 Interior & 13.84 & 69.96 & 16.61 & \\
\hline $\mathrm{AP}$ & 3-3 Interior & 12.74 & 65.1 & 15.48 & \\
\hline $\mathrm{AP}$ & 3-4 Interior & 8 & 35.13 & 9.53 & \\
\hline $\mathrm{AP}$ & 3-4 Interior & 7.46 & 33.02 & 9.08 & \\
\hline $\mathrm{AP}$ & 3-4 Interior & 7.07 & 34.14 & 8.61 & \\
\hline $\mathrm{AP}$ & 4-1 Interior & 10.64 & 52.15 & 12.75 & \\
\hline $\mathrm{AP}$ & 4-1 Interior & 11.63 & 61.08 & 14.42 & \\
\hline $\mathrm{AP}$ & 4-1 Interior & 11.76 & 56.6 & 14.53 & \\
\hline $\mathrm{AP}$ & 4-2 Interior & 12.28 & 65.62 & 15.39 & \\
\hline $\mathrm{AP}$ & 4-2 Interior & 12.45 & 58.42 & 15.51 & \\
\hline $\mathrm{AP}$ & 4-2 Interior & 16.88 & 83.15 & 21.21 & \\
\hline $\mathrm{AP}$ & 4-3 Interior & 13.94 & 74.21 & 17.03 & \\
\hline $\mathrm{AP}$ & 4-3 Interior & 11.93 & 65.62 & 14.89 & \\
\hline $\mathrm{AP}$ & 4-3 Interior & 13.63 & 68.84 & 16.65 & \\
\hline $\mathrm{AP}$ & 4-4 Interior & 13.11 & 68.76 & 16 & \\
\hline $\mathrm{AP}$ & 4-4 Interior & 13.48 & 70.2 & 16.61 & \\
\hline $\mathrm{AP}$ & 4-4 Interior & 15.25 & 76.06 & 18.21 & \\
\hline $\mathrm{AP}$ & 5-1 Interior & 12.06 & 63.86 & 15.11 & \\
\hline $\mathrm{AP}$ & 5-1 Interior & 11.99 & 61.84 & 14.93 & \\
\hline $\mathrm{AP}$ & 5-1 Interior & 10.88 & 51.77 & 13.28 & \\
\hline $\mathrm{AP}$ & 5-2 Interior & 10.43 & 52.4 & 12.71 & \\
\hline
\end{tabular}




\begin{tabular}{|l|l|l|l|l|l|}
\hline AP & 5-2 Interior & 11.1 & 60.43 & 13.9 & \\
\hline AP & 5-2 Interior & 10.96 & 57.39 & 13.61 & \\
\hline AP & 5-3 Interior & 14.06 & 74.32 & 17.6 & \\
\hline AP & 5-3 Interior & 13.79 & 77.59 & 17.23 & \\
\hline AP & 5-3 Interior & 16.44 & 76.46 & 19.51 & \\
\hline AP & 5-4 Interior & 14.97 & 71.29 & 17.73 & \\
\hline AP & 5-4 Interior & 18.27 & 89.86 & 22.7 & \\
\hline AP & 5-4 Interior & 16.65 & 78.5 & 19.59 & \\
\hline AP & 6-1 Interior & 9.77 & 52.77 & 12.16 & \\
\hline AP & 6-1 Interior & 11.77 & 57.8 & 14.77 & \\
\hline AP & 6-1 Interior & 13.47 & 60.97 & 16.11 & \\
\hline AP & 6-2 Interior & 12.66 & 62.88 & 15.68 & \\
\hline AP & 6-2 Interior & 12.89 & 60.15 & 15.23 & \\
\hline AP & 6-2 Interior & 12.45 & 64.96 & 15.45 & \\
\hline AP & 6-3 Interior & 14.68 & 76.13 & 17.94 & \\
\hline AP & 6-3 Interior & 14.2 & 74.71 & 17.21 & \\
\hline AP & 6-3 Interior & 14.08 & 73.13 & 17.32 & \\
\hline AP & 6-4 Interior & 15.76 & 81.34 & 19.33 & \\
\hline AP & 6-4 Interior & 17.6 & 87.56 & 20.92 & \\
\hline AP & 6-4 Interior & 15.32 & 77.74 & 18.34 & \\
\hline AP & Big O Top & 28.4 & 135.9 & 33.62 & \\
\hline AP & Big O Top & 20.6 & 102.4 & 25.24 & \\
\hline AP & Big O Top & 24.99 & 115.5 & 29.71 & \\
\hline AP & Big O Bot & 8.838 & 44.38 & 10.5 & \\
\hline AP & Big O Bot & 11.3 & 50.3 & 13.7 & \\
\hline AP & Big O Bot & 8.9 & 52.29 & 12.18 & \\
\hline AP & Small O Top & 26.59 & 128.6 & 32.43 & \\
\hline AP & Small O Top & 17.86 & 93.2 & 21.66 & \\
\hline AP & Small O Top & 23.87 & 118.1 & 29.29 & \\
\hline AP & Small O bot & 13.63 & 65.64 & 16.97 & \\
\hline AP & Small O bot & 9.45 & 52.48 & 12.51 & \\
\hline AP & Small O bot & 8.31 & 52.94 & 11.41 & \\
\hline & & & & & \\
\hline
\end{tabular}

Angle and Radius Measurements for As Printed and Electropolished

\begin{tabular}{|l|l|l|l|l|l|l|}
\hline EP Angle & AP Angle & ID & $\begin{array}{l}\text { EP } \\
\text { radius }\end{array}$ & As Printed radius & Hole & Corner \\
\hline 89.4 & 91.94 & $\begin{array}{l}\text { Arc 1- } \\
\text { 1a: } \\
\text { Radius }\end{array}$ & 0.2876 & 0.71755 & 1 & 1 \\
\hline 91.16 & 95.42 & $\begin{array}{l}\text { Arc 1- } \\
\text { 2a: } \\
\text { Radius }\end{array}$ & 0.3023 & 0.8116824 & 1 & 2 \\
\hline
\end{tabular}




\begin{tabular}{|c|c|c|c|c|c|c|}
\hline 89.94 & 91.7 & $\begin{array}{l}\text { Arc 1- } \\
\text { 3a: } \\
\text { Radius }\end{array}$ & 0.4444 & 0.5427472 & 1 & 3 \\
\hline 97.21 & 93.11 & $\begin{array}{l}\text { Arc 1- } \\
\text { 4a: } \\
\text { Radius }\end{array}$ & 0.2668 & 0.541147 & 1 & 4 \\
\hline 91.35 & 87.6 & $\begin{array}{l}\text { Arc 2- } \\
\text { 1a: } \\
\text { Radius }\end{array}$ & 0.4202 & 0.4399788 & 2 & 1 \\
\hline 97.33 & 94.87 & $\begin{array}{l}\text { Arc 2- } \\
\text { 2a: } \\
\text { Radius }\end{array}$ & 0.228 & 0.3843528 & 2 & 2 \\
\hline 89.29 & 93.68 & $\begin{array}{l}\text { Arc 2- } \\
\text { 3a: } \\
\text { Radius }\end{array}$ & 0.5104 & 0.7929372 & 2 & 3 \\
\hline 96.39 & 92.06 & $\begin{array}{l}\text { Arc 2- } \\
\text { 4a: } \\
\text { Radius }\end{array}$ & 0.1754 & 0.352171 & 2 & 4 \\
\hline 88.16 & 90.49 & $\begin{array}{l}\text { Arc 3- } \\
\text { 1a: } \\
\text { Radius }\end{array}$ & 0.5528 & 0.59182 & 3 & 1 \\
\hline 91.72 & 85.84 & $\begin{array}{l}\text { Arc 3-2: } \\
\text { Radius }\end{array}$ & 0.274 & 0.5341874 & 3 & 2 \\
\hline 87.05 & 96.3 & $\begin{array}{l}\text { Arc 3- } \\
\text { 3a: } \\
\text { Radius }\end{array}$ & 0.704 & 0.7837424 & 3 & 3 \\
\hline 94.84 & 90.19 & $\begin{array}{l}\text { Arc 3- } \\
\text { 4a: } \\
\text { Radius }\end{array}$ & 0.2214 & 0.4479544 & 3 & 4 \\
\hline 90.48 & 89.15 & $\begin{array}{l}\text { Arc 4- } \\
\text { 1a: } \\
\text { Radius }\end{array}$ & 0.3204 & 0.2509774 & 4 & 1 \\
\hline 94 & 95.02 & $\begin{array}{l}\text { Arc 4- } \\
\text { 2a: } \\
\text { Radius }\end{array}$ & 0.2972 & 0.1767332 & 4 & 2 \\
\hline 93.96 & 92.13 & $\begin{array}{l}\text { Arc 4- } \\
\text { 3a: } \\
\text { Radius }\end{array}$ & 0.5569 & 0.2854198 & 4 & 3 \\
\hline 90.06 & 100.26 & $\begin{array}{l}\text { Arc 4- } \\
\text { 4a: } \\
\text { Radius }\end{array}$ & 0.3061 & 0.256286 & 4 & 4 \\
\hline 89.61 & 87.53 & $\begin{array}{l}\text { Arc 5- } \\
\text { 1a: } \\
\text { Radius }\end{array}$ & 0.2889 & 0.168529 & 5 & 1 \\
\hline 96.31 & 92.81 & $\begin{array}{l}\text { Arc 5- } \\
\text { 2a: } \\
\text { Radius }\end{array}$ & 0.3034 & 0.4570222 & 5 & 2 \\
\hline 98.23 & 96.25 & $\begin{array}{l}\text { Arc 5- } \\
\text { 3a: } \\
\text { Radius }\end{array}$ & 0.5187 & 0.4150868 & 5 & 3 \\
\hline 93.52 & 94.08 & $\begin{array}{l}\text { Arc 5- } \\
\text { 4a: } \\
\text { Radius }\end{array}$ & 0.346 & 0.5285486 & 5 & 4 \\
\hline
\end{tabular}




\begin{tabular}{|c|c|c|c|c|c|c|}
\hline 92.5 & 91.7 & $\begin{array}{l}\text { Arc 6- } \\
\text { 1a: } \\
\text { Radius }\end{array}$ & 0.4977 & 0.5735066 & 6 & 1 \\
\hline 92.59 & 96.12 & $\begin{array}{l}\text { Arc 6- } \\
\text { 2a: } \\
\text { Radius }\end{array}$ & 0.3661 & 0.515493 & 6 & 2 \\
\hline 95.24 & 93.96 & $\begin{array}{l}\text { Arc 6- } \\
\text { 3a: } \\
\text { Radius }\end{array}$ & 0.6431 & 0.7962392 & 6 & 3 \\
\hline 95.79 & 95.93 & $\begin{array}{l}\text { Arc 6- } \\
\text { 4a: } \\
\text { Radius }\end{array}$ & 0.3058 & 0.4140962 & 6 & 4 \\
\hline 91.8 & 89.68 & $\begin{array}{l}\text { Arc 7- } \\
\text { 1a: } \\
\text { Radius }\end{array}$ & 0.3565 & 0.1922018 & 7 & 1 \\
\hline 88.34 & 90.2 & $\begin{array}{l}\text { Arc 7- } \\
\text { 2a: } \\
\text { Radius }\end{array}$ & 0.346 & 0.1540002 & 7 & 2 \\
\hline 92.64 & 97.8 & $\begin{array}{l}\text { Arc 7- } \\
\text { 3a: } \\
\text { Radius }\end{array}$ & 0.5441 & 0.1615694 & 7 & 3 \\
\hline 96.19 & 93.57 & $\begin{array}{l}\text { Arc 7- } \\
\text { 4a: } \\
\text { Radius }\end{array}$ & 0.2226 & 0.1007618 & 7 & 4 \\
\hline 93.02 & 89.6 & $\begin{array}{l}\text { Arc 8- } \\
\text { 1a: } \\
\text { Radius }\end{array}$ & 0.3591 & 0.201803 & 8 & 1 \\
\hline 91.51 & 93.12 & $\begin{array}{l}\text { Arc 8- } \\
\text { 2a: } \\
\text { Radius }\end{array}$ & 0.2867 & 0.0962152 & 8 & 2 \\
\hline 94.74 & 71.05 & $\begin{array}{l}\text { Arc 8- } \\
\text { 3a: } \\
\text { Radius }\end{array}$ & 0.503 & 0.287147 & 8 & 3 \\
\hline 96.01 & 94.46 & $\begin{array}{l}\text { Arc 8- } \\
\text { 4a: } \\
\text { Radius }\end{array}$ & 0.276 & 0.1194562 & 8 & 4 \\
\hline 87.66 & 97.32 & $\begin{array}{l}\text { Arc 9- } \\
\text { 1a: } \\
\text { Radius }\end{array}$ & 0.4448 & 0.196088 & 9 & 1 \\
\hline 93.12 & 92.33 & $\begin{array}{l}\text { Arc 9- } \\
\text { 2a: } \\
\text { Radius }\end{array}$ & 0.4076 & 0.0950468 & 9 & 2 \\
\hline 91.53 & 86.81 & $\begin{array}{l}\text { Arc 9- } \\
\text { 3a: } \\
\text { Radius }\end{array}$ & 0.7114 & 0.278892 & 9 & 3 \\
\hline 98.14 & 95.31 & $\begin{array}{l}\text { Arc 9- } \\
\text { 4a: } \\
\text { Radius }\end{array}$ & 0.2348 & 0.1388364 & 9 & 4 \\
\hline 89.47 & 91.65 & $\begin{array}{l}\text { Arc 1- } \\
\text { 1a: } \\
\text { Radius }\end{array}$ & 0.3192 & 0.7514082 & 1 & 1 \\
\hline 91.1 & 91.5 & $\begin{array}{l}\text { Arc 1- } \\
\text { 2a: } \\
\text { Radius }\end{array}$ & 0.3044 & 0.7516622 & 1 & 2 \\
\hline
\end{tabular}




\begin{tabular}{|c|c|c|c|c|c|c|}
\hline 89.7 & 96.77 & $\begin{array}{l}\text { Arc 1- } \\
\text { 3a: } \\
\text { Radius }\end{array}$ & 0.4683 & 0.6094476 & 1 & 3 \\
\hline 96.29 & 93.11 & $\begin{array}{l}\text { Arc 1- } \\
\text { 4a: } \\
\text { Radius }\end{array}$ & 0.2851 & 0.5873496 & 1 & 4 \\
\hline 91.08 & 96.05 & $\begin{array}{l}\text { Arc 2- } \\
\text { 1a: } \\
\text { Radius }\end{array}$ & 0.4269 & 0.4812792 & 2 & 1 \\
\hline 98.55 & 89.14 & $\begin{array}{l}\text { Arc 2- } \\
\text { 2a: } \\
\text { Radius }\end{array}$ & 0.2254 & 0.3279648 & 2 & 2 \\
\hline 94.81 & 95.33 & $\begin{array}{l}\text { Arc 2- } \\
\text { 3a: } \\
\text { Radius }\end{array}$ & 0.4687 & 0.7767828 & 2 & 3 \\
\hline 96.09 & 92.48 & $\begin{array}{l}\text { Arc 2- } \\
\text { 4a: } \\
\text { Radius }\end{array}$ & 0.2265 & 0.3792728 & 2 & 4 \\
\hline 91.77 & 92.11 & $\begin{array}{l}\text { Arc 3- } \\
\text { 1a: } \\
\text { Radius }\end{array}$ & 0.4721 & 0.3510788 & 3 & 1 \\
\hline 92.86 & 88.02 & $\begin{array}{l}\text { Arc 3-2: } \\
\text { Radius }\end{array}$ & 0.2699 & 0.4674362 & 3 & 2 \\
\hline 86.04 & 105.88 & $\begin{array}{l}\text { Arc 3- } \\
\text { 3a: } \\
\text { Radius }\end{array}$ & 0.7144 & 0.8123174 & 3 & 3 \\
\hline 94.99 & 89.37 & $\begin{array}{l}\text { Arc 3- } \\
\text { 4a: } \\
\text { Radius }\end{array}$ & 0.2202 & 0.4593336 & 3 & 4 \\
\hline 89.14 & 87.87 & $\begin{array}{l}\text { Arc 4- } \\
\text { 1a: } \\
\text { Radius }\end{array}$ & 0.3012 & 0.1264666 & 4 & 1 \\
\hline 95.34 & 98.48 & $\begin{array}{l}\text { Arc 4- } \\
\text { 2a: } \\
\text { Radius }\end{array}$ & 0.3206 & 0.1798574 & 4 & 2 \\
\hline 94.44 & 90.93 & $\begin{array}{l}\text { Arc 4- } \\
\text { 3a: } \\
\text { Radius }\end{array}$ & 0.5561 & 0.427228 & 4 & 3 \\
\hline 90.7 & 101.23 & $\begin{array}{l}\text { Arc 4- } \\
\text { 4a: } \\
\text { Radius }\end{array}$ & 0.312 & 0.2571242 & 4 & 4 \\
\hline 89.85 & 87.14 & $\begin{array}{l}\text { Arc 5- } \\
\text { 1a: } \\
\text { Radius }\end{array}$ & 0.2843 & 0.498221 & 5 & 1 \\
\hline 95.63 & 90.72 & $\begin{array}{l}\text { Arc 5- } \\
\text { 2a: } \\
\text { Radius }\end{array}$ & 0.2882 & 0.4403598 & 5 & 2 \\
\hline 98.71 & 103.51 & $\begin{array}{l}\text { Arc 5- } \\
\text { 3a: } \\
\text { Radius }\end{array}$ & 0.5102 & 0.424053 & 5 & 3 \\
\hline 94.37 & 95.39 & $\begin{array}{l}\text { Arc 5- } \\
\text { 4a: } \\
\text { Radius }\end{array}$ & 0.3701 & 0.7215124 & 5 & 4 \\
\hline
\end{tabular}




\begin{tabular}{|c|c|c|c|c|c|c|}
\hline 91.77 & 94 & $\begin{array}{l}\text { Arc 6- } \\
\text { 1a: } \\
\text { Radius }\end{array}$ & 0.4752 & 0.5967984 & 6 & 1 \\
\hline 91.67 & 94.38 & $\begin{array}{l}\text { Arc 6- } \\
\text { 2a: } \\
\text { Radius }\end{array}$ & 0.3617 & 0.5401564 & 6 & 2 \\
\hline 95.56 & 96.85 & $\begin{array}{l}\text { Arc 6- } \\
\text { 3a: } \\
\text { Radius }\end{array}$ & 0.6448 & 0.8707882 & 6 & 3 \\
\hline 96.41 & 87.45 & $\begin{array}{l}\text { Arc 6- } \\
\text { 4a: } \\
\text { Radius }\end{array}$ & 0.3099 & 0.4363212 & 6 & 4 \\
\hline 91.81 & 84.41 & $\begin{array}{l}\text { Arc 7- } \\
\text { 1a: } \\
\text { Radius }\end{array}$ & 0.3747 & 0.1512062 & 7 & 1 \\
\hline 88.49 & 99.91 & $\begin{array}{l}\text { Arc 7- } \\
\text { 2a: } \\
\text { Radius }\end{array}$ & 0.3367 & 0.1317498 & 7 & 2 \\
\hline 92.37 & 86.75 & $\begin{array}{l}\text { Arc 7- } \\
\text { 3a: } \\
\text { Radius }\end{array}$ & 0.5689 & 0.247396 & 7 & 3 \\
\hline 98.23 & 93.21 & $\begin{array}{l}\text { Arc 7- } \\
\text { 4a: } \\
\text { Radius }\end{array}$ & 0.2496 & 0.1509268 & 7 & 4 \\
\hline 92.42 & 85.67 & $\begin{array}{l}\text { Arc 8- } \\
\text { 1a: } \\
\text { Radius }\end{array}$ & 0.3523 & 0.268605 & 8 & 1 \\
\hline 91.49 & 95.49 & $\begin{array}{l}\text { Arc 8- } \\
\text { 2a: } \\
\text { Radius }\end{array}$ & 0.3028 & 0.1068832 & 8 & 2 \\
\hline 91.02 & 72.33 & $\begin{array}{l}\text { Arc 8- } \\
\text { 3a: } \\
\text { Radius }\end{array}$ & 0.5033 & 0.282448 & 8 & 3 \\
\hline 94.98 & 92.07 & $\begin{array}{l}\text { Arc 8- } \\
\text { 4a: } \\
\text { Radius }\end{array}$ & 0.2514 & 0.1474216 & 8 & 4 \\
\hline 88.12 & 87.13 & $\begin{array}{l}\text { Arc 9- } \\
\text { 1a: } \\
\text { Radius }\end{array}$ & 0.4561 & 0.4645914 & 9 & 1 \\
\hline 92.54 & 93.17 & $\begin{array}{l}\text { Arc 9- } \\
\text { 2a: } \\
\text { Radius }\end{array}$ & 0.4425 & 0.1022858 & 9 & 2 \\
\hline 92.57 & 86.84 & $\begin{array}{l}\text { Arc 9- } \\
\text { 3a: } \\
\text { Radius }\end{array}$ & 0.6847 & 0.363347 & 9 & 3 \\
\hline 97.33 & 88.57 & $\begin{array}{l}\text { Arc 9- } \\
\text { 4a: } \\
\text { Radius }\end{array}$ & 0.2317 & 0.1976882 & 9 & 4 \\
\hline 89.2 & 91.91 & $\begin{array}{l}\text { Arc 1- } \\
\text { 1a: } \\
\text { Radius }\end{array}$ & 0.3112 & 0.7356 & 1 & 1 \\
\hline 91.32 & 90.7 & $\begin{array}{l}\text { Arc 1- } \\
\text { 2a: } \\
\text { Radius }\end{array}$ & 0.3052 & 0.7768 & 1 & 2 \\
\hline
\end{tabular}




\begin{tabular}{|c|c|c|c|c|c|c|}
\hline 89.21 & 77.97 & $\begin{array}{l}\text { Arc 1- } \\
\text { 3a: } \\
\text { Radius }\end{array}$ & 0.4646 & 0.2981 & 1 & 3 \\
\hline 96.82 & 92.99 & $\begin{array}{l}\text { Arc 1- } \\
\text { 4a: } \\
\text { Radius }\end{array}$ & 0.2653 & 0.5731 & 1 & 4 \\
\hline 90.93 & 89.51 & $\begin{array}{l}\text { Arc 2- } \\
\text { 1a: } \\
\text { Radius }\end{array}$ & 0.4247 & 0.4822 & 2 & 1 \\
\hline 98.31 & 89.55 & $\begin{array}{l}\text { Arc 2- } \\
\text { 2a: } \\
\text { Radius }\end{array}$ & 0.2281 & 0.3692 & 2 & 2 \\
\hline 91.83 & 96.45 & $\begin{array}{l}\text { Arc 2- } \\
\text { 3a: } \\
\text { Radius }\end{array}$ & 0.4952 & 0.7811 & 2 & 3 \\
\hline 96.28 & 92.56 & $\begin{array}{l}\text { Arc 2- } \\
\text { 4a: } \\
\text { Radius }\end{array}$ & 0.206 & 0.3676 & 2 & 4 \\
\hline 91.13 & 90.82 & $\begin{array}{l}\text { Arc 3- } \\
\text { 1a: } \\
\text { Radius }\end{array}$ & 0.4656 & 0.3996 & 3 & 1 \\
\hline 93.06 & 79.04 & $\begin{array}{l}\text { Arc 3-2: } \\
\text { Radius }\end{array}$ & 0.2773 & 0.4351 & 3 & 2 \\
\hline 85.29 & 89.73 & $\begin{array}{l}\text { Arc 3- } \\
\text { 3a: } \\
\text { Radius }\end{array}$ & 0.6986 & 0.8234 & 3 & 3 \\
\hline 94.55 & 88.49 & $\begin{array}{l}\text { Arc 3- } \\
\text { 4a: } \\
\text { Radius }\end{array}$ & 0.2177 & 0.4169 & 3 & 4 \\
\hline 89.1 & 90.37 & $\begin{array}{l}\text { Arc 4- } \\
\text { 1a: } \\
\text { Radius }\end{array}$ & 0.3087 & 0.1668 & 4 & 1 \\
\hline 94.81 & 87.35 & $\begin{array}{l}\text { Arc 4- } \\
\text { 2a: } \\
\text { Radius }\end{array}$ & 0.3289 & 0.2269 & 4 & 2 \\
\hline 94.46 & 91.75 & $\begin{array}{l}\text { Arc 4- } \\
\text { 3a: } \\
\text { Radius }\end{array}$ & 0.5546 & 0.3481 & 4 & 3 \\
\hline 90.91 & 98.21 & $\begin{array}{l}\text { Arc 4- } \\
\text { 4a: } \\
\text { Radius }\end{array}$ & 0.3075 & 0.2864 & 4 & 4 \\
\hline 89.42 & 87.48 & $\begin{array}{l}\text { Arc 5- } \\
\text { 1a: } \\
\text { Radius }\end{array}$ & 0.2871 & 0.5302 & 5 & 1 \\
\hline 96.13 & 87.68 & $\begin{array}{l}\text { Arc 5- } \\
\text { 2a: } \\
\text { Radius }\end{array}$ & 0.2791 & 0.1675 & 5 & 2 \\
\hline 98.42 & 94.43 & $\begin{array}{l}\text { Arc 5- } \\
\text { 3a: } \\
\text { Radius }\end{array}$ & 0.5305 & 0.5017 & 5 & 3 \\
\hline 94.19 & 99.79 & $\begin{array}{l}\text { Arc 5- } \\
\text { 4a: } \\
\text { Radius }\end{array}$ & 0.3715 & 0.6171 & 5 & 4 \\
\hline
\end{tabular}




\begin{tabular}{|c|c|c|c|c|c|c|}
\hline 92.07 & 92.77 & $\begin{array}{l}\text { Arc 6- } \\
\text { 1a: } \\
\text { Radius }\end{array}$ & 0.4724 & 0.5783 & 6 & 1 \\
\hline 93.15 & 95.43 & $\begin{array}{l}\text { Arc 6- } \\
\text { 2a: } \\
\text { Radius }\end{array}$ & 0.3875 & 0.1074 & 6 & 2 \\
\hline 95.56 & 97.31 & $\begin{array}{l}\text { Arc 6- } \\
\text { 3a: } \\
\text { Radius }\end{array}$ & 0.6398 & 0.1167 & 6 & 3 \\
\hline 96.4 & 95.35 & $\begin{array}{l}\text { Arc 6- } \\
\text { 4a: } \\
\text { Radius }\end{array}$ & 0.3094 & 0.4316 & 6 & 4 \\
\hline 91.71 & 87.43 & $\begin{array}{l}\text { Arc 7- } \\
\text { 1a: } \\
\text { Radius }\end{array}$ & 0.38 & 0.1756 & 7 & 1 \\
\hline 88.18 & 96.59 & $\begin{array}{l}\text { Arc 7- } \\
\text { 2a: } \\
\text { Radius }\end{array}$ & 0.3452 & 0.1505 & 7 & 2 \\
\hline 92.41 & 105.11 & $\begin{array}{l}\text { Arc 7- } \\
\text { 3a: } \\
\text { Radius }\end{array}$ & 0.581 & 0.1924 & 7 & 3 \\
\hline 96.62 & 92.8 & $\begin{array}{l}\text { Arc 7- } \\
\text { 4a: } \\
\text { Radius }\end{array}$ & 0.2231 & 0.1611 & 7 & 4 \\
\hline 92.53 & 84.25 & $\begin{array}{l}\text { Arc 8- } \\
\text { 1a: } \\
\text { Radius }\end{array}$ & 0.3687 & 0.8137 & 8 & 1 \\
\hline 91.48 & 92.22 & $\begin{array}{l}\text { Arc 8- } \\
\text { 2a: } \\
\text { Radius }\end{array}$ & 0.2906 & 0.1341 & 8 & 2 \\
\hline 90.31 & 74.28 & $\begin{array}{l}\text { Arc 8- } \\
\text { 3a: } \\
\text { Radius }\end{array}$ & 0.5169 & 0.2821 & 8 & 3 \\
\hline 95.19 & 91.72 & $\begin{array}{l}\text { Arc 8- } \\
\text { 4a: } \\
\text { Radius }\end{array}$ & 0.2569 & 0.1068 & 8 & 4 \\
\hline 88.03 & 91.97 & $\begin{array}{l}\text { Arc 9- } \\
\text { 1a: } \\
\text { Radius }\end{array}$ & 0.4575 & 0.1538 & 9 & 1 \\
\hline 92.89 & 79.18 & $\begin{array}{l}\text { Arc 9- } \\
\text { 2a: } \\
\text { Radius }\end{array}$ & 0.3856 & 0.1057 & 9 & 2 \\
\hline 92.28 & 79.68 & $\begin{array}{l}\text { Arc 9- } \\
\text { 3a: } \\
\text { Radius }\end{array}$ & 0.6943 & 0.2021 & 9 & 3 \\
\hline 97.93 & 91.1 & $\begin{array}{l}\text { Arc 9- } \\
\text { 4a: } \\
\text { Radius }\end{array}$ & 0.225 & 0.1714 & 9 & 4 \\
\hline
\end{tabular}

